\title{
DIGITAL GENRE: A MECHANISM FOR KNOWLEDGE SHARING AND REUSE IN BUSINESS CLUSTERS
}

By

María Eugenia Molina Barrantes

\begin{abstract}
A thesis
submitted to the Victoria University of Wellington in fulfilment of the requirements for the degree of Doctor of Philosophy in Information Systems Management
\end{abstract}

VICTORIA UNIVERSITY OF WELLINGTON 
This thesis is dedicated to my parents

Maru and Julio 


\begin{abstract}
The purpose of this study was to identify the knowledge transfer mechanisms in project bidding for two business clusters in New Zealand, and how ICT played a role in facilitating a "virtual space" for sharing and re-use of these mechanisms. Genre Systems was the theoretical framework adopted to guide this inquiry and to build on further theory.
\end{abstract}

Within the context of this study, genres are the knowledge transfer mechanisms that communicate information and knowledge to members of the community, following specific social rules. The genres and the way they are being employed contribute towards explaining how knowledge is shared and reused by a community.

Action research methodology was used to direct data collection and analysis, and validate how the study was carried out. The study comprised of one action research cycle, which has been divided in five stages: Diagnosis, Planning, Development, Evaluation, and Specifying Learning.

Mapping the clusters' collaborative interactions during project bidding helped to identify the knowledge transfer mechanisms. This allowed the identification of an ICT solution that could improve project bidding, and identification of how this knowledge could be stored for reuse in future bids.

One of the clusters decided to work together with the researcher towards the design of a new portal to address their project bidding needs. The portal took six iterations to complete and went live in November 2005. A database, several "digital genres" (since these genres exist in an electronic medium), and some procedures were created to facilitate knowledge transfer for the cluster's project bidding process. The team had the opportunity to reflect on the whole experience, identify potential features and genres to incorporate in the portal, and start thinking how they could improve the development process in future interventions.

The introduction of ICT encouraged the cluster to develop digital genres that were more dynamic and flexible than the ones used before then. The main finding of the study is a five-step process to create digital genres based on the activities carried out by the team: finding reference points for the digital genre; defining the social rules for the digital genre; embedding the social rules in the template; testing the template; and legitimising the digital genre.

Further findings discussed the "natural" and "induced" ways for a cluster to increase its knowledge-base. The first instance takes place during the normal practices of cluster members working together towards business opportunities over a period of time, whereas the later instance is triggered by a specific event or initiative. In this study, the decision by the cluster to introduce website and database technology to assist in managing their knowledge-base provided an opportunity to explore the role of ICT in increasing the cluster's knowledge-base.

Final findings showed that a project of this nature not only has to overcome the common IT development challenges (budget, project management, user buy-in), but also those derived from working with a team of volunteer people from different organisations, such as in the case of a cluster. 


\section{Acknowledgements}

I would like to acknowledge Natural Hazards New Zealand (NHNZ) and Earthquake Engineering New Zealand (EENZ) business clusters for agreeing to be the subjects of this study, and for letting me become part of their community over three years. I am very grateful to the Executives of both clusters for granting me their consent to name the clusters in my thesis. Being able to disclose the identity of the clusters has allowed me to provide a richer context and hence gives major credibility to the study.

My thanks also go to members of the Website and Content Task Groups who participated in the intervention stages of this study, which led to a successful launch of the portal for NHNZ in November 2005. In particular, I would like to express my appreciation to NHNZ Co-chairs and the Facilitator for their leadership and enthusiasm, which ensured the completion of the project.

The assistance from Positively Wellington Business - the then Wellington's regional economic development agency - in introducing me to NHNZ and EENZ is also acknowledged.

I am deeply grateful to my supervisor Professor Pak Yoong for his guidance, mentorship, and friendship throughout this academic journey. He is very passionate about how ICT can facilitate collaboration and knowledge sharing among groups in the business environment, and thus he understood my aspiration to carry out research where I could work closely with practitioners.

Being an experienced action researcher in the Information Systems domain, Professor Yoong made sure I did not fall into the "trap" of focusing too much on solving the practical problem and not contributing to the enhancement of knowledge, or vice versa.

During my studies I formed part of a small PhD Study Group at the School of Information Management. The PhD Study Group was a forum where we shared experiences about our doctoral research. I extend my appreciation to my fellow students who were part of this community for their enthusiasm in developing knowledge about doing qualitative research.

I am grateful to the School of Information Management (SIM) of Victoria University of Wellington. SIM provided me with office space, printing, and photocopying facilities to assist in the writing of this thesis. SIM academic and administrative staff was always welcoming and helpful. For many years SIM was my second home.

I would like to acknowledge the financial support for three years of my studies granted by Victoria University of Wellington through their Scholarship Programme.

I extend my gratitude to my employer, the Institute of Geological and Nuclear Sciences, for granting me time from work to complete the writing of this thesis. My thanks also go to my previous manager Mr Rob Johnston, my current manager $\mathrm{Mr}$ Tony Charles, and fellow work colleagues Dr Noel Trustrum and Dr Hannah Brackley for their constant encouragement.

Finally, I would like to acknowledge the immense support and love that I received from my family in New Zealand and in Costa Rica. I am grateful to my husband David, who not only looked after me during my student years, but also patiently proof-read many drafts of this thesis. 


\title{
Table of contents
}

\author{
Abstract, i \\ Acknowledgements, ii \\ Table of contents, iii \\ List of tables, viii \\ Table of figures, ix
}

\section{Chapter 1: Introduction, 1}

1.1. Statement of the problem, 1

1.2. Purpose of the study, 2

1.3. The research questions, 2

1.4. Delimitations of the study, 3

1.5. Significance of the study, 4

1.6. Organisation of the dissertation, 5

\section{PART 1: THE RESEARCH DESIGN, 6}

\section{Chapter 2: Literature review, 7}

2.1. The business environment, 7

2.1.1. Knowledge Economy: the "new" paradigm, 8

2.1.2. Companies in the Knowledge Economy, 9

2.2. Clusters, 11

2.2.1. The concept of cluster in the literature, 12

2.2.2. The formation of clusters, 14

2.2.3. The composition of a cluster, 15

2.2.4. Cluster development, 17

2.2.5. The role of clusters in the Knowledge Economy, 21

2.3. Knowledge and its transfer in clusters, 22

2.3.1. The concept of knowledge, 23

2.3.2. Knowledge in clusters, 26

2.3.3. Knowledge transfer, 28

2.3.4. Two aspects of knowledge transfer: sharing and reuse, 30

2.3.4.1. Knowledge sharing, 30

2.3.4.2. Knowledge reuse, 32

2.3.5. Knowledge transfer mechanisms, 37

2.4. Information and Communication Technologies (ICT), 41

2.4.1. The role of ICT in knowledge transfer, 42

2.4.2. ICT supporting knowledge transfer in clusters, 43

2.5. Formulating the study's research questions, 45

\section{Chapter 3: The theoretical framework, 50}

3.1. The value of a theoretical framework, 50

3.2. Theoretical frameworks considered for the study, 52

3.2.1. Rolland and Chauvel's knowledge transfer framework, 52 
3.2.2. Tallman, Jenkins, Henry and Pinch's knowledge-base framework, 54

3.3. The genre theory framework, 56

3.4. Genre repertoire and genre systems, 59

3.5. The Genre framework in Information Systems (IS), 62

3.6. Using genre theory as the theoretical framework for this study, 65

\section{Chapter 4: Philosophical Position and Methodology, 68}

4.1. The philosophical paradigm of this study, 68

4.1.1. The philosophical paradigms in IS inquiry, 68

4.1.2. Advocacy/participatory as the study's philosophical paradigm, 72

4.2. Selecting the study's research methodology, 73

4.3. The action research methodology, 74

4.3.1. Action research in the literature, 75

4.3.1.1. Close to context, 77

4.3.1.2. Action and change, 77

4.3.1.3. Involvement, 77

4.3.1.4. Cyclical nature, 78

4.3.1.5. Ability to address practice and theory, 81

4.3.2. Action research in information systems (IS), 82

4.3.3. Variants of action research in the IS literature, 86

4.3.4. Rationale for using action research in this study, 88

4.4. The study's action research design, 89

4.4.1. Canonical action research as the selected stream, 89

4.4.2. The research site, 90

4.4.3. Entry to the site, 91

4.4.4. The role of the researcher, 93

4.4.5. The role of participants, 95

4.4.6. Data collection procedures, 96

4.4.7. Data analysis procedures, 100

4.4.8. Exiting the site, 104

4.4.9. The narrative of the study, 105

\section{PART 2: THE ACTION RESEARCH INTERVENTION, 106}

\section{Chapter 5: The context of the study, 107}

5.1. The national economic and political context, 107

5.2. The regional context, 109

5.3. Earthquake Engineering NZ and Natural Hazards NZ clusters, 112

\section{Chapter 6: The Diagnosis Stage, 115}

6.1. Executing the Diagnosis Stage, 115

6.2. Project bidding in EENZ and NHNZ, 116

6.3. The proactive and reactive approaches in project bidding, 117

6.4. Indentifying the genres in project bidding, 117 


\section{Chapter 7: The Planning Stage, 120}

7.1. The "old" NHNZ website, 120

7.2. The purpose of the new NHNZ website, 123

7.3. Preparing the NHNZ Specifications Document, 124

7.4. Discussion Planning Stage, 128

\section{Chapter 8: The Development Stage, 131}

8.1. Preceding Step: selecting the web developer for NHNZ Portal, 132

8.1.1. What happened in the Preceding Step, 132

8.1.2. Discussion Preceding Step, 133

8.2. First Iteration: developing the first prototype of NHNZ Portal, 134

8.2.1. What happened in the First Iteration, 134

8.2.2. Discussion First Iteration, 137

8.3. Second Iteration: developing the second prototype of NHNZ Portal, 138

8.3.1. What happened in the Second Iteration, 138

8.3.2. Discussion Second Iteration, 140

8.4. Third Iteration: Developing the third prototype of NHNZ portal, 141

8.4.1. What happened in the Third Iteration, 141

8.4.2. Discussion Third Iteration, 145

8.5. Fourth Iteration: Developing the fourth prototype of NHNZ Portal, 146

8.5.1. What happened in this fourth iteration, 146

8.5.2. Discussion Fourth Iteration, 151

8.6. Fifth Iteration: Developing the final version of NHNZ Portal, 152

8.6.1. What happened in this Fifth Iteration, 152

8.6.2. Discussion Fifth Iteration, 158

\section{Chapter 9: The Portal Evaluation Stage, 159}

9.1. Systems design evaluation, 159

9.2. Project evaluation, 161

9.3. Participant evaluation, 162

9.3.1. Subscribing to the Task Group approach, 164

9.3.2. Learning about system design, 164

9.3.3. The cluster knowledge-base, 165

9.3.4. The use of the resources in the Portal for project bidding, 166

9.3.5. What can be done to improve the NHNZ Portal, 167

9.3.6. The challenges for the new Portal, 168

\section{PART 3: SPECIFYING LEARNING STAGE* ${ }^{*} 169$}

\section{Chapter 10: The Project Bidding System, 170}

10.1. The reactive project bidding approach, 170

10.2. The proactive project bidding approach, 179

\footnotetext{
* Specifying Learning is the last stage of the action research cyclical model I followed in my study; however, for reporting purposes I have separated it from the rest of the stages.
} 
10.3. Mapping the knowledge transfer mechanisms, 182

10.4. Areas where ICT could support project bidding, 184

\section{Chapter 11: The Process of digitising genres, 187}

11.1. Three digital genres in the NHNZ Project Bidding system, 187

11.1.1. Expert Profile, 188

11.1.2. Project Sheet, 188

11.1.3. Company Profile, 189

11.2. The process of creating digital genres, 189

11.2.1. Finding reference point for digital genre, 190

11.2.2. Defining the social rules for the digital genre, 191

11.2.3. Embedding social rules in the online forms and electronic templates, 194

11.2.4. Testing the digital genre, 195

11.2.5. Legitimising the digital genre, 197

\section{Chapter 12: The development of a cluster's knowledge-base on project bidding, 199}

12.1. The cluster's "natural" growth of its knowledge-base, 199

12.2. The cluster's "induced" growth of its knowledge-base, 203

12.3. The role of the Portal in increasing the cluster's knowledge-base, 205

\section{Chapter 13: Factors that influence the growth of the cluster's knowledge-base, 209}

13.1. Small budget, 210

13.2. Committed volunteers, 211

13.3. Active champions, 213

13.4. The presence of a project coordinator, 214

13.5. Trigger event, 215

13.6. Supportive Economic Development Agency, 216

13.7. Level of up-take by cluster members, 217

\section{PART 4: DISCUSSION AND CONCLUSION, 219}

\section{Chapter 14: Considerations associated with the study, 220}

14.1. Considerations when executing the action research study, 220

14.1.1. Outcome failure, 221

14.1.2. Availability of participants, 224

14.1.3. Issues of power, 225

14.1.4. Managing expectations, 227

14.2. Ethical considerations, 228

14.2.1. Confidentiality, 228

14.2.2. Remaining neutral, 228

14.2.3. Anonymity, 228 
14.3. Considerations about the credibility of the study, 229

14.3.1. Internal validity, 230

14.3.2. Recoverability, 231

14.3.3. Generalisability/transferability, 231

\section{Chapter 15: Conclusion, 232}

15.1. Revisiting the research questions, 233

15.2. Discussion of findings, 235

15.3. Contributions to knowledge, 238

15.4. Implications for practice, 240

15.5. Directions for future research, 241

15.6. Update, 241

\section{References, 244}

\section{Acronyms, 268}

\section{Appendixes, 269}

Appendix A: Interview tools, 270

Appendix B: Project evaluation tool, 273

Appendix C: Reactive project bidding process for EENZ and NHNZ - Description of the activities involved during the process, 274

Appendix D: Proactive project bidding process for EENZ and NHNZ - Description of the activities involved during the process, 279

Appendix E: Genre Systems Analysis for EENZ-NHNZ Project Bidding, 284 


\section{List of Tables}

Table 1. Types of knowledge at firm and cluster level (Tallman et al., 2004), 55

Table 2. Three Genre Systems (Yates \& Orlikowski, 2002), 61

Table 3. Working definition of action research (Altrichter et al., 2002), 76

Table 4. Forms of action research in IS (Baskerville \& Wood-Harper, 1998), 87

Table 5. Data collection techniques for qualitative methods (Creswell, 2003; McNiff, 1988), 98

Table 6. The genres found in the sub-system "Prepare EOI", 118

Table 7. The four underlying risks that could lead to outcome failure (Baskerville, 2001), 222 


\section{Table of figures}

Figure 1. The anatomy of a cluster (Ffocws-William, 1997), 16

Figure 2. Life-cycle of an industrial cluster (Su \& Hung, 2009), 17

Figure 3. Cluster development process (Gray et al., 2001), 18

Figure 4. Factors influencing inter-organisational knowledge transfer (EasterbySmith et al., 2008), 28

Figure 5. Knowledge sharing mechanisms (Appleyard, 1996), 38

Figure 6. Decision tree to explore the appropriate knowledge transfer mechanism (Jasimuddin, 2007), 39

Figure 7. A model of knowledge transfer (Rolland \& Chauvel, 2000), 52

Figure 8. The evolution of cybergenres (Sheperd \& Watters, 1998), 63

Figure 9. The action research cyclical model (Baskerville \& Wood-Harper, 1996), 79

Figure 10. The core action research and thesis action research cycle (Zuber-Skerritt \& Perry, 2002), 82

Figure 11. The Wellington region (www.localcouncils.govt.nz), 110

Figure 12. NHNZ’s “old” Homepage (www.naturalhazards.co.nz), 121

Figure 13. Risk and Disaster Management page from the old NHNZ's website (www.naturalhazards.co.nz), 122

Figure 14. Proposed web pages and navigation from new Homepage, 125

Figure 15. Proposed web pages and navigation to see the projects, 126

Figure 16. The new NHNZ Portal's Homepage (www.naturalhazards.co.nz), 157

Figure 17. The reactive project bidding process followed by NHNZ and EENZ, 171

Figure 18. The proactive project bidding process followed by NHNZ and EENZ, 180

Figure 19. The process of creating digital genres, 190

Figure 20. The cluster's knowledge-base on project bidding, 200

Figure 21. Development of cluster's project bidding knowledge-base over time, 202

Figure 22. NHNZ's knowledge-base after the intervention, 205

Figure 23. Level of intermediation by ICT due to the interventions, 207

Figure 24. Factors influencing a cluster's knowledge-base, 209

Figure 25. Current NHNZ Portal's Homepage (www.naturalhazards.co.nz), 243 


\section{Chapter 1: Introduction}

Clusters are geographical concentrations of firms in related industries that share needs for human resources, technology, and infrastructure (Waits, 2000). Clusters have been identified as vehicles to generate innovative and knowledge-based products and services that can be exported and thus contribute to strengthening a country or region's competitive position (Bailey, Koziarski \& Knight, 2002; Parto, 2008). In addition, Bailey et al. (2002) indicated that clusters are viewed as a forum where ideas and experiences are shared.

A cluster that is conscious of its potential will organise itself to combine the capabilities and competencies of its independent firms in order to access international market opportunities, address industry-wide issues, exchange expertise, and achieve economies of scale (Bailey et al., 2002). In this way firms are prepared to share knowledge, even when their partners may be direct competitors in other spheres.

This study is concerned with the way information and communication technologies (ICT) can support a cluster's knowledge sharing and reuse during their project bidding process. More specifically, it looks at how to improve pertinent knowledge transfer mechanisms - the means through which knowledge is exchanged. The following sections will explain the problem, purpose, research question, delimitations, and significance of the study.

\subsection{Statement of the problem}

Tricker (1992) argued that firms have a rich stock of knowledge that is under-utilised due to being dispersed, not available in the right form, or often consisting of individuals' unrecorded impressions and insights. The same could be said about clusters.

The problem is not a lack of knowledge stock in clusters - they create and transfer information and knowledge when their firms link up with one another to collaborate and co-ordinate their efforts - but how clusters can manage their knowledge to share it more effectively among their firms and with offshore partners/clients. Clusters usually form teams from their members to bid for a particular project or a contract, but when the 
work is over the team is dispersed. The documentation, lessons learnt, and experience generated by the team could be reused and built upon by others in later projects.

A cluster does not always have the appropriate mechanisms and systems to ensure that its knowledge-base is readily available. With knowledge being considered a valuable competitive advantage (Davenport \& Prusak, 1998; ITAG, 1999), it is critical that clusters improve their knowledge-base to remain a key innovation source. Consequently, the cluster must work on its organisational memory to collect, preserve, and manage what has been learnt.

ICT can play a fundamental role in facilitating knowledge creation and transfer in a cluster by providing fast, reliable, and cheap means of communication and collaboration, data collection and storage, and information management. In order to develop effective ICT systems it is therefore important to discover how ICT can support or enhance knowledge creation and transfer in some key cluster processes or activities.

\subsection{Purpose of the study}

The purpose of this study is two-fold: firstly to identify the knowledge mechanisms in the business activity of project bidding in the international arena, commonly undertaken by clusters in Wellington, New Zealand; and secondly to explore the role of ICT in facilitating a "virtual space" for exchange and reuse of these knowledge mechanisms.

\subsection{The research questions}

Two primary research questions addressed in the study are:

1) What are the knowledge transfer mechanisms in a cluster when it performs a business activity such as international project bidding?

2) How can ICT be used to improve cluster knowledge transfer during project bidding? 


\subsection{Delimitations of the study}

My study followed a qualitative methodology, giving careful consideration to the context in which the phenomenon took place and the views of the research participants. These activities demanded considerable time and resources for satisfactory completion (Creswell, 2003). For this reason I concentrated on two clusters based in Wellington, namely Natural Hazards New Zealand and Earthquake Engineering New Zealand. This allowed the establishment of trusting relationships with the participants and an understanding of the clusters' dynamics.

The study does not provide in-depth analysis on the nature of the clusters that were studied, analyse their inter-firm networks to identify the relationships among the cluster members, or assess the quality of their knowledge-base, all topics that are of interest to scholars and practitioners in regional economic development and planning. Instead, the study takes an information systems view of how ICT can assist clusters in knowledge transfer and in developing their knowledge transfer mechanisms.

The study focused in looking at the knowledge transfer mechanisms used in one key business process: international project bidding. The decision to concentrate on this process was made after consultation with the clusters regarding areas in which they felt knowledge mechanisms should be improved.

I used a qualitative research methodology known as action research to address the research questions. In action research methodology, the researcher and participants collaborate in the analysis of a problem within a social context (in this case, the cluster) to produce two outcomes: a solution to the practical problem, and the enhancement of knowledge. To achieve this, researcher and practitioners make assumptions based on their observation of the problem, and formulate actions to change the status quo (Baskerville, 1999).

Addressing the practical problem of this study involved the development of a new website for one of the clusters, and thus the participants and I followed web-based information systems development approaches and project management principles. Enhancement of knowledge in these two domains was not the focus of the study, and consequently the literature on theories and models concerning web design and project management was not extensively explored. Nevertheless, supplementary findings associated with these fields as a result of the intervention are reported in Chapter 13. 
I carried out one action research cycle (diagnosis, planning, development, evaluation, and specifying learning). Given the nature of the clusters and how they operate. I was constrained by time and resources from performing further cycles in this study.

Finally, the scope of this research did not include the development of a tool for evaluating the effectiveness of the proposed knowledge mechanisms. Instead, the comments and feedback of the participants provided an indication of the usefulness of the new system.

\subsection{Significance of the study}

There is a growing interest by theorists and practitioners in the subject of clusters. This study, however, departed from the usual economic development perspective that dominates the cluster literature. It focused instead on cluster knowledge management and the role of ICT in supporting such tasks. Analysing the knowledge mechanisms in project bidding activities led to the identification of factors that affect knowledge creation and knowledge transfer within the cluster. This in turn confirmed and even expanded on some propositions found in the cluster literature, such as Bailey et al., (2002) and Molina and Yoong (2003).

The study was research in practice. It provided the opportunity to observe a small number of clusters and learn how they perceived and employed ICT for knowledge creation and transfer activities.

A key output of this study was the development of a theory to explain how the knowledge-base in clusters can be shaped to enable sharing and re-use, and how ICT can support this. The theory was the outcome of describing, qualifying, and interpreting practice (Markus, 1997). This was consistent with García and Quek's (1997) argument that information systems research is about "improving the effectiveness of information systems design, implementation and use in organisations, and to assess their impact on individuals, organisations and society at large" (p. 450).

The clusters participating in the study gained increased awareness of their own knowledge-base. Through a better understanding of the dynamics of knowledge creation and knowledge transfer in their international project bidding process, and the way ICT can support them, cluster members and some of their stakeholders were better able to develop procedures to maintain their knowledge-base. 


\subsection{Organisation of the dissertation}

In this chapter I outlined the problem, purpose, research question, methodology delimitations, and significance of the study. The rest of this dissertation has been divided into four parts to assist the reader.

Part One covers the design of the study and includes Chapters 2, 3, and 4. Chapter 2 provides contextual information about the current business environment, clusters, knowledge transfer, and ICT. The theoretical framework that guided my inquiry is proposed in Chapter 3. Chapter 4 explains my philosophical stance and describes action research as the methodology for this study.

Part Two contains Chapters 5, 6, 7, 8, and 9. Chapter 5 describes the context of the study, while the remaining chapters detail the action research intervention. Each chapter covers the first four stages of the action research cycle: diagnosis, planning, development (action taking), and evaluation.

Part Three outlines the last stage of action research (specifying learning). The findings of the study are detailed in Chapters 10, 11, 12, and 13.

Part Four supplies further discussions and my conclusions. Chapter 13 provides some considerations associated with the study. Finally, Chapter 14 summarises the conclusions of this study. 


\section{PART 1:}

\section{THE RESEARCH DESIGN}

Part 1 sets the stage of this research enquiry. To provide the context of the study, I put forward a summary of the literature concerned with economical environment, clusters, knowledge, knowledge transfer, and ICT that led to the formulation of the research questions. I propose the theoretical framework used as the starting point of this enquiry, and the methodology followed in my efforts to enhance knowledge in the Information Systems domain. 


\section{Chapter 2: Literature review}

This Chapter provides an overview of the business and economical environment in which clusters are embedded. It also introduces the concepts of clusters, knowledge and its transfer, and the role of ICT. These concepts are then linked together to give the background information for this study. The literature review has guided me in formulating the study's enquiry.

\subsection{The business environment}

Firms develop strategies to address the challenge of surviving and thriving in a constantly changing business environment. Such strategies are based on a way of perceiving the world - in other words, a paradigm.

The production and organisational paradigm that dominated most of the $20^{\text {th }}$ century aimed to increase the speed and volume of material flowing through production. The focus was on the design and integration of complex routines and processes to achieve economies of time (Best, 1990; Wielemaker, Elfring \& Volberda, 2001).

Competition was carried out by the premises of reducing costs and expanding supply by lowering prices, which had the potential for "cut-throat" price wars. Best (1990) and Porter (1990) agree that, by the 1970s, firms in industrialised countries were suffering deterioration in their world export manufacturing leadership. Both authors concur that two main reasons for such deterioration were: a) low-wage countries catching up in mass production methods, and b) high-wage countries starting to compete based on product quality and innovation.

Best (1990), Davenport and Prusak (1998), Drucker (1994), and Sveiby (1997) believe that, by the end of the $20^{\text {th }}$ century, qualitative and quantitative changes in the social, economic, and political aspects of our societies had affected the environment where businesses interact and a new paradigm was taking these changes into account: the Knowledge Economy.

This section will first describe the Knowledge Economy. After that, attention will be paid to how firms are adjusting to face the challenges of this new paradigm. 


\subsubsection{Knowledge Economy: the "new" paradigm}

In The Competitive Advantage of Nations, Porter (1990) identified three types of changes in today's business environment that have nullified the advantages of some nations: a) technology, b) factor endowments, and c) globalisation.

\section{a) Technology}

The OECD (1998) reported that members' economies were experiencing technological change in the form of a high rate of science discoveries, new patents, and the dissemination of information and communication technologies. For Porter (1990) and Romer (1990), technology changes enable firms to overcome shortages or improve the use of productive factors through the development of new materials, products, or processes.

With technology, nations have managed to reduce the advantage enjoyed by other nations richer in productive factors. Porter (1990) explains how Sweden's advantage in possessing low-phosphorus iron ores was undercut once technology created a viable method for eliminating impurities in iron. Similarly, "catch-up economies" such as Ireland, Korea, and Mexico have improved their productivity and growth by incorporating international technologies into their economic activities (OECD, 1998).

\section{b) Factor endowments}

Pearlson and Saunders (2004) indicate that a large percentage of economic growth originates from knowledge-based companies, which require less traditional factors such as land, labour, and capital. In addition, Porter (1990) argues that many developing countries have invested in improving their productive factors, such as up-skilling their human resources through education and developing their infrastructure (e.g.roads, telecommunications, and ports). Hence, these countries are becoming world-class competitors. An example is South Korea, which has caught up to industrialised nations and now competes internationally in fields such as consumer electronics and cars (Magaziner \& Patinkin, 1989; Porter, 1990).

\section{c) Globalisation}

Firms are now competing in a global environment (Davenport \& Prusak, 1998; Simmie, 2003). For the OECD (1998), business activities such as selling, sourcing components, buying materials, approaching investors, and finding business partners are now taking place worldwide. This is due not only to deregulation in the 
financial and product markets, but also to low telecommunication costs and the availability of information and communication technologies (ICTs). In addition, Bryant (2003) points out that globalisation is not only about economic transactions of goods and services, but is also about the exchange of knowledge and culture across political boundaries.

The opening of new markets allows companies to place their products in new territories, with a tendency towards price reduction in commodity goods. Globalisation also frees firms from relying solely on the factors endowments of a particular nation for the raw materials and components required to produce their goods and services, enabling them to locate anywhere in the world the suppliers that best meet their needs and terms (Crocombe, Enright \& Porter, 1991). At the same time, globalisation brings additional competition to firms, as consumers have a wider range from which to choose and they may opt to purchase goods and services produced abroad (Davenport \& Prusak, 1998).

These changes in technology, factors endowments, and globalisation have caused a review of the economic model. Many authors called the emerging paradigm “Knowledge Economy” (Davenport \& Prusak, 1998; ITAG, 1999; Probst, Raub \& Romhardt, 2000), as there is a consensus that value is being added to products and services through knowledge and information.

\subsubsection{Companies in the Knowledge Economy}

One important aspect pointed out by Bergquist, Betwee, and Meuel (1995), and Sveiby (1997), is the fact that firms in the Knowledge Economy recognise they are not isolated systems. Firms are now looking outside their boundaries for people and organisations consumers, vendors, suppliers, competitors, employees, and the community - to help them achieve their competitive goals more efficiently and effectively. Furthermore, Sveiby (1997) observed that such reaching out was based not so much on financial flows, but rather on knowledge and information flows.

Individual firms constitute parts of a larger system which supplies and supports other firms, customers, policies, and the environment. Therefore a firm's competitiveness will depend not only on its internal strategy and organisation, but also on external agents. A firm could improve its position through activities such as exchange of ideas, dialogue, co-ordination, and negotiation with external agents (Bryant, 2003). 
Regarding strategies for improving flexibility to enable organisational structures to adjust quickly to change, Drucker (1998) and DeFillipi (2002) mention working teams that are assembled and dissolved according to the requirements of a particular project. These teams may be formed from people from various departments or units within the organisation, or may even cross organisational boundaries to include staff from suppliers, customers, and contracted third parties.

The incorporation of ICT into the firm's strategic direction is also being considered by firms in the Knowledge Economy. In the production arena, ICT combined with the right organisational change has maximised efficiency, flexibility, and productivity at company level (OECD, 1998; Yates \& Benjamin, 1991). The Internet and e-commerce have introduced new business models to satisfy consumer's demands for better value, according to their needs, as cheaply as possible, and at "any time, any place" (El Sawy, Malhotra, Gosain \& Young, 1999; DeFillipi, 2002). In the financial markets ICT has reduced transaction costs to capital mobility (OECD, 1998). Finally, lower prices in ICT have accelerated the distribution of codified knowledge (Jonscher, 1994; OECD, 1998).

Another approach explored by organisations is to focus on those activities in which they already have a competitive advantage - or can easily obtain it - and pass those activities they are not good at on to others (Van de Ven, 2005). The idea is to develop the firm's core business, build trusting relationships with partners, and become a node in a value chain network. Furthermore, Roelandt and den Hertog (1999) argue that networks should allow firms to access new and complementary knowledge and to accelerate their learning process, as these interactions stimulate innovation.

The opening of new markets through globalisation or technological advancements exposes companies to potentially profitable ventures. However, some business opportunities in these new markets cannot be tackled by any firm on its own due to the risk involved, financial demands, or expertise required. The strategy opted for by companies in these cases is to collaborate with other companies that are also their direct competitors (Kluge, Stein \& Licht, 2001; Loebbecke, Van Fenema \& Powell, 1999). Such an arrangement is what Nalebuff and Brandenburger (1996) call co-opetition - a term coined by Novell founder Ray Noorda. Nalebuff and Brandenburger (1996) explain that firms can cooperate to create a larger or new market, but at the same time these firms will compete with each other over how to divide such markets. 
Nalebuff and Brandenburger (1996) also argue that it is short-sighted to perceive competitors only as "enemies." There are situations when working with competitors is desirable in order to develop common complements. As an example, the authors mention how hi-tech competitors join forces to build infrastructure and standards in IT, such as the case of IBM, Sun, Compaq, Cisco, Netscape, Oracle, and others, who in August 1996 formed a $\$ 100$ million venture capital fund to promote Java technology as the next standard for network computing.

According to Nalebuff and Brandenburger (1996), co-opetition can explain why competing businesses are found nearby, such as the diamond merchants at $47^{\text {th }}$ Street in New York, or the antiquarian bookstores in London. By "bunching together," these firms became "complementors" of each other, as consumers find it convenient to travel to one location knowing that there are several options from which to choose, or that their chances of finding exactly what they want are increased. Similarly, suppliers (i.e. labour, components, and services) are attracted to cater for the critical mass created by a high concentration of similar firms.

Companies of the $21^{\text {st }}$ century are expected to be flexible and cope with the Knowledge Economy context whilst continuing to be profitable. This is a significant challenge, particularly for established companies, because it involves changing a company's values, beliefs, attitudes, norms, and practices: in other words, its organisational culture (Best, 1990). In addition, firms are challenged to see outside their boundaries and view their competitors as allies with whom to engage in a new business opportunity. This is not a spontaneous process and it demands commitment at every level of the organisation.

\subsection{Clusters}

By "bunching together," competing firms create the critical mass needed to attract customers, suppliers, skilled labour, and providers of complementary services into a particular geographic location. This section will explore the concept of the cluster as one way of co-opetition in the Knowledge Economy paradigm. It will firstly introduce some of the common terms found in the literature that have been used interchangeably with cluster. The following sub-sections will explain how clusters are formed, examining their composition and how they usually evolve. Finally, this section will explain the role of a cluster in the Knowledge Economy paradigm. 


\subsubsection{The concept of cluster in the literature}

There are many terms found in the literature that have been used interchangeably with cluster, such as agglomeration, business network, industrial district, industrial clusters, and regional clusters (Maskell, 2001; McDonald \& Vertova, 2001; Simmie \& Sennett, 1999). This can lead to some confusion on this subject by the neophyte reader, as authors may use different terms when talking about the same phenomena, or employ the same term for two different concepts. For instance, the apparel manufacturers in Carpi, Italy are referred as clusters by Ffowcs-Williams (1997), while Brioschi, Brioschi and Cainelli (2002) used the term industrial district. What matters here is that the reader is aware of the different emphasis each term carries, how it has been employed in a specific situation, and how to relate it to other studies.

For authors like McDonald and Vertova (2001) and Simmie and Sennett (1999), the term cluster describes a spatial agglomeration of similar firms. The mere proximity of these firms seems to facilitate cost reductions, knowledge transfer, and access to capital infrastructure, what Gordon and McCann (2000) defined as "external economies." Consequently, there is no implication that firms link and engage in formal collaborative processes or long-term relations. However, results from quantitative studies like Rosenthal and Strange (2003) have provided evidence that agglomerating is insufficient; it seems that industrial structure and corporate organisation are two other dimensions that affect the benefits from clustering and are worth exploring further.

A business network is described by Braun, Gaebe, Grotz, Okamoto and Yamamoto (2002) and Giuliani (2007) as firms maintaining ongoing relations with each other, which usually lead to a certain degree of collective learning and innovation processes. Through networking, firms are able to carry out economic activities to generate sales and profits, such as exchange of information, joint production, marketing, and exporting, while reducing the costs and risks associated with the development of a new product or service (Rosenfeld, 1997; UNCTAD, 1997). Firms, however, do not need to be from related industries to form a business network; neither are they required to be geographically concentrated. Therefore, when the term business network is used in a cluster context, it is focusing on the links and relationships between some partners within the cluster rather than on their close proximity.

The concept of the industrial district has its origins in the work of Marshall in the late 1800s and early 1900s (McDonald \& Vertova, 2001; Parto, 2008), who noted that small 
and locally-owned firms grouped together for investment and production decisions. Local labour in an industrial district is highly specialised and flexible, where workers move from firm to firm but seldom leave the region. Support services are also specialised and tailored to the specific requirements of the district.

According to Markusen (1996), the district is viewed as a stable community with a strong local cultural identity and shared industrial expertise. The UNTAD (1997) also indicate that industrial districts are "locally coordinated and well-articulated economic systems" (p.5). Italian industrial districts have been extensively studied (Lorenzoni \& Ferriani, 2008; Muscio, 2006).

Studies from North America (Romanelli and Khessina, 2005; Waits, 2000), Australia (Roberts \& Enright, 2004) and New Zealand (Lindsay, 2005) tend to use the term industrial clusters. Industrial cluster depicts a concentration of competitive firms of a specialised industry in a geographical location that interact with each other, usually in a value-chain relationship to produce innovative goods and services (Roberts \& Enright, 2004; Waits, 2000). Gordon and McCann (2000) characterised clusters under this model as privately organised systems, whose members have incurred capital and/or research costs to set up their trading links.

Regional cluster encompasses the features of the industry cluster, but emphasises the geographic proximity of the firms rather than the common economic activity shared by these firms (Enright \& Roberts, 2001). In other words, while industry cluster may imply a focus of the cluster on a single economic industry - or even a segment of it - regional cluster embodies a range of related industries found within a region. Most of the literature that refers to regional cluster tends to focus on competitiveness, policy, or economic development at the regional level, such as the work by Asheim and Isaksen (2002), Enright and Roberts (2001), and Park and Lee (2004).

For the purpose of this literature review, the term cluster will be used in a broad sense in order to tap into the literature that uses any of these definitions. This will allow me to provide an overall picture of cluster formation, composition, life-cycle, and how they contribute in the Knowledge Economy. In Chapter Five, where the Natural Hazards New Zealand and the Earthquake Engineering New Zealand clusters - the subjects of the study - are introduced, I have provided specific details about them. 


\subsubsection{The formation of clusters}

Although the method and reasons for some clusters successfully appearing in certain locations cannot be totally resolved, McDonald and Vertova (2001) point out three sets of conditions they believe influence the formation of clusters: geographical factors, historical events, and institutional frameworks. A fourth condition proposed by Lorenzoni and Ferriani (2008) is the presence of change agents.

\section{a) Geographical factors}

According to Roberts and Enright (2004), and McDonald and Vertova (2001), geographical factors provide economic reasons for clustering. Firms may concentrate in particular geographical locations in order to exploit a favourable climate or unique raw materials for the production of their goods, like the case of the seafood industry in the Nelson region of New Zealand mentioned by Pavlovich and Akoorie (2005). Clusters may also emerge near target markets in order to take advantage of the proximity to their consumers or suppliers, especially if haulage costs are high or if the product is difficult to transport.

Firms may enjoy reduced transaction costs, as well as improved communications and support services, when doing business with partners located nearby (Enright \& Roberts, 2001; Porter, 1998b). Helmsing (1999) indicated that specific business values and codes may emerge when local firms start interacting frequently with each other. Such values can minimise "bad deals" as firms would not engage in opportunistic behaviour when working with nearby partners, in order to avoid the risk of gaining a bad reputation in the region (Enright \& Roberts, 2001) and becoming a "local outcast" (Maskell, 2001:926).

\section{b) Historical events}

Historical events make up the second group of factors influencing cluster development. In this case, events in the past influence the location of the cluster today (Porter, 1998b). McDonald and Vertova (2001) provide the example of the advantage gained by London as a financial centre when the US government in the 1950s prohibited US banks from denominating overseas loans in dollars.

\section{c) Institutional Frameworks}

McDonald and Vertova (2001) describe institutional frameworks as "the legalpolitical-social structures that define the rules of human interaction" (p.159). In 
addition, they argue that such frameworks can be of formal (constitutions, bills, courts) or informal (cultural norms, generally accepted attitudes) nature.

Institutional frameworks are developed by national or local governments to shape the transaction costs, and thus attract businesses to settle in their region (McDonald \& Vertova, 2001; Roberts \& Enright, 2004). Such frameworks may facilitate the business environment by cutting down bureaucratic steps, thus fast-forwarding the establishment of a business in a region, and may even contribute to minimising risks associated with the business activities. An example of successful clusters being conceived in this form is found in the software industry in Dublin, Ireland.

\section{d) Change agents}

Lorenzoni and Ferriani (2008) propose that a small group of leading actors, through their deliberate actions, contribute to the formation of clusters in a particular location. Entrepreneurs who financially support new businesses fall within this category.

Although any of these factors is capable of triggering the clustering process by itself, in practice a combination of them usually initiates events. For instance, the formation of Silicon Valley was influenced by the historical presence of Stanford University, the geographical concentration of computer scientists, and the interest of communities and local government in boosting the cluster into what it is now.

\subsubsection{The composition of a cluster}

Broadly speaking, a cluster is described as having four layers (Ffowcs-Williams 1996; Waits 2000): the core, the specialist supporting firms, the supporting social infrastructure firms, and the physical infrastructure (see Figure 1). 


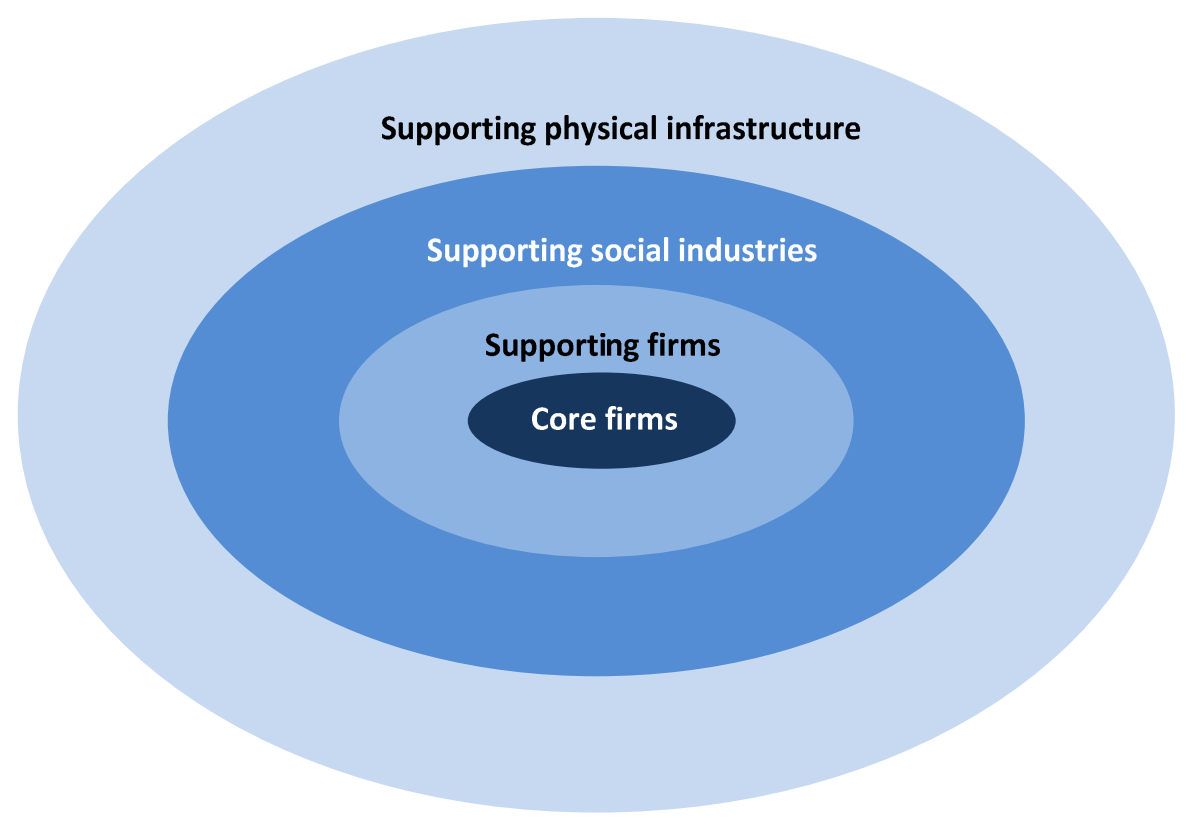

Figure 1. The anatomy of a cluster (source: Ffowcs-Williams, 1997).

The core of a cluster comprises of the highly specialised group of similar or related organisations that produce the primary service or product of the cluster. The second layer consists of firms that provide supplies or specialised services to those organisations at the core. Banks, law firms, insurance companies, and component suppliers are examples for this layer. The third layer includes educational institutions, training organisations, business and technology advisors, and professional and trading associations. Organisations in this layer are responsible for up-skilling the workforce for the cluster's needs. The last layer contains the physical infrastructure and the respective organisations that provide it, such as transport, R\&D capabilities, waste disposal, telecommunications, and other utilities. For Ffowcs-Williams (1997), a successful cluster integrates all these separate elements and creates a sense of "specialised ecosystem."

Lorenzoni and Ferriani (2008) argue that this ecosystem view assumes that "everybody works with everybody" (p.127) in a cluster, which is inconsistent with empirical findings. In addition, they state that clusters as units of analysis cannot provide details on how firms organised themselves, how they collaborated with one another, and how they tapped into their sources of innovation and knowledge. This is why more recent cluster research focuses on the network capacity of the firms within the cluster, rather than the cluster itself. 


\subsubsection{Cluster development}

Clusters vary in size, depth, configuration, and level of development (Helmsing, 1999; Porter, 1998a). In addition, clusters take time to form (OECD, 1999). Based on case studies, Porter (1998a) estimates that a cluster may require at least ten years of development before becoming an effective source of competitive advantage.

Lindsay (2005) argues that recent literature proposes two views about cluster formation and development: one which she labels "inertial view," where the evolutionary path of a cluster ends in its decline; and the "coevolutionary view," where a cluster achieves long-term sustainability by evolving with its changing environment.

Figure 2 is the conceptual model used by Su and Lung (2009) ${ }^{1}$ to depict the life-cycle of an industrial cluster based on the work by the Cleverbio Project ${ }^{2}$ (Chiesa \& Chiaroni, 2005), which fits Lindsay's (2005) description of the inertial view. The model encompasses three stages: origin, growth, and decline/reorientation.

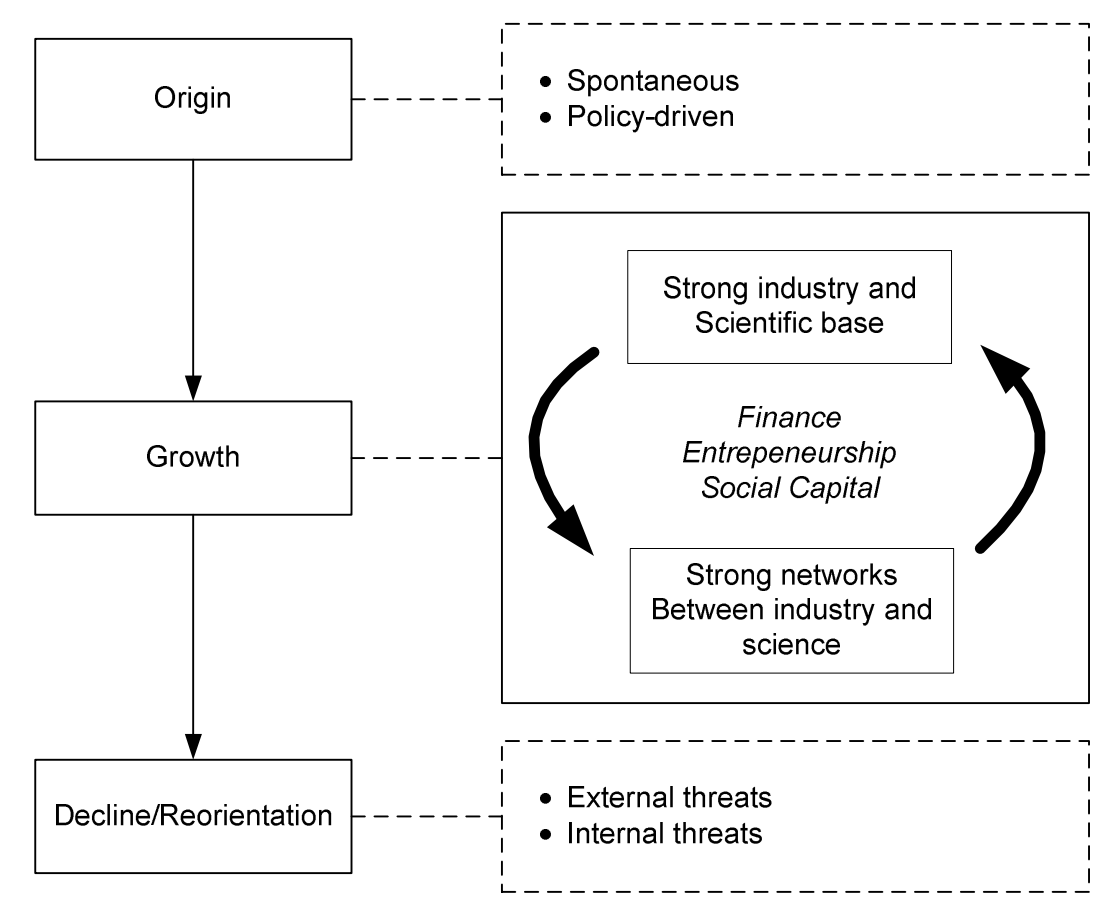

Figure 2. Life-cycle of an industrial cluster (source: Su \& Hung, 2009).

\footnotetext{
${ }^{1}$ This paper was retrieved during the revision process of the thesis. I include it as it provides an example of a cluster development model following the "inertial" approach.

${ }^{2}$ The Cleverbio Project (Cluster development and growth in bio-tech: enabling factors and best practice) was funded by the European Commission with the objective of defining a normative model for cluster approach in bio-technology.
} 
As discussed, some clusters are associated with the presence of particular natural resources in a region, while others have historical or political roots. For $\mathrm{Su}$ and Lung (2009) the origin of these clusters can be described as spontaneous or policy-driven. During the growth stage, the interactions between the cluster's science and industry base, the finance supporting mechanisms (pre-seed capital, seed capital, venture capital, and government funds), its entrepreneurs, and the networking links allow the cluster to self-reinforce and prosper. The decline/reorientation stage acknowledges that clusters lose their competitive edge over time as a result of external (technology discontinuities, shift in buyers' needs, etc.) and internal (loss of innovative abilities, high degree of homogeneity within the cluster, and static mental models) threats.

The co-evolutionary view, on the other hand, is based on the premise that clusters are "complex adaptive systems" (Lindsay, 2005:73) with intricate internal and external relationships that are constantly accessed for new knowledge in order to maintain the cluster's innovation and performance as it evolves with the environment. In their report for Industry New Zealand (now known as New Zealand Trade and Enterprise), Gray, Harvey, and Brimblecombe (2001) outline a development model for clusters targeting the international market that aligns with the co-evolutionary view (Figure 3). The model depicts four key stages: initiation, incubation, implementation, and improvement.

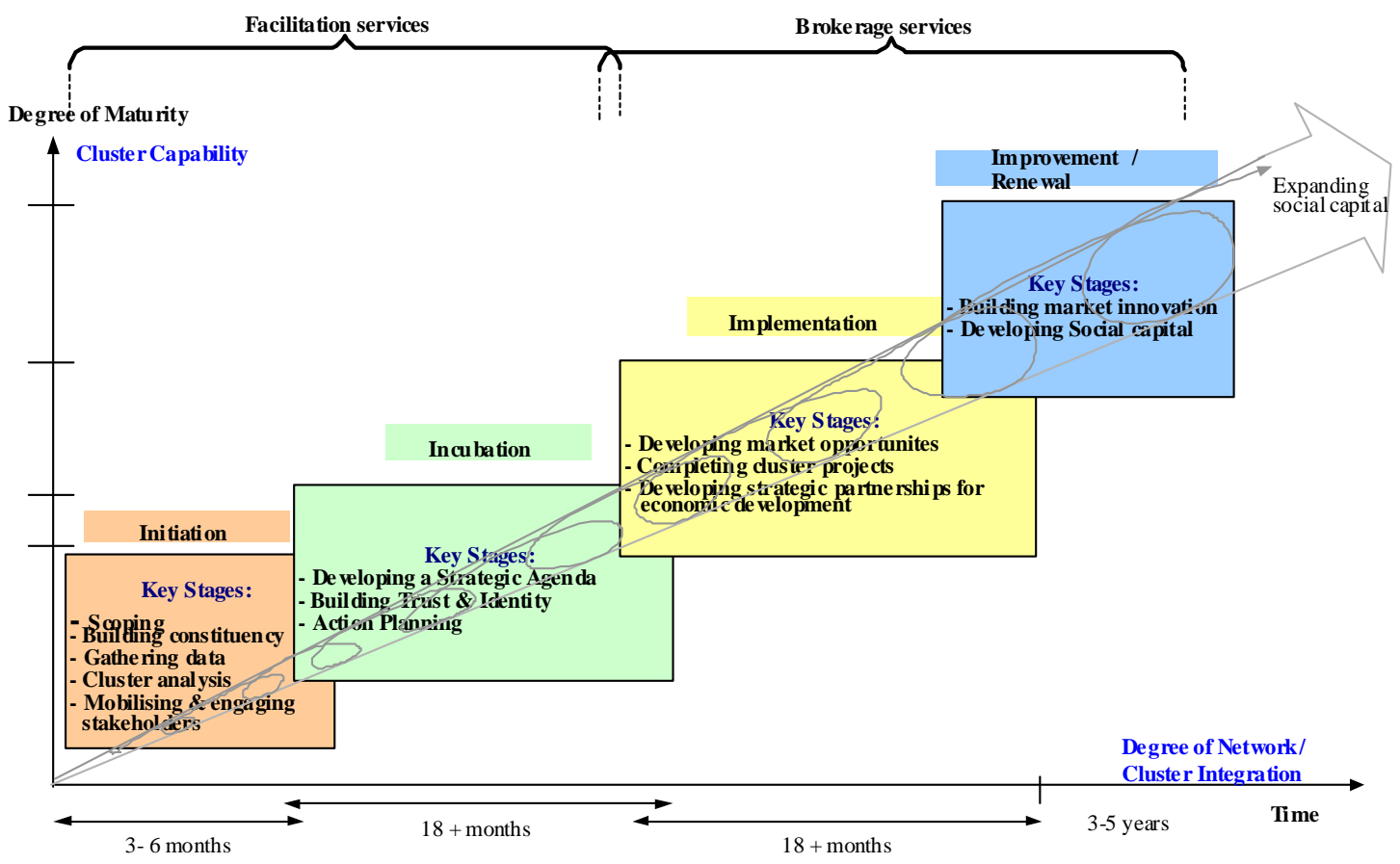

Figure 3. Cluster development process (source: Gray et al., 2001). 
In the initiation stage, the cluster potential is identified. Companies gather together in an informal manner to discuss the possibility of establishing a cluster. There is initial support from relevant economic development agencies, Government branches, research centres, and universities. Potential cluster members identify the cluster's strengths, weaknesses, business opportunities, and threats.

During the incubation stage, the task of bringing cluster members together continues. The cluster has a defined common vision, objectives, and strategic plan. Formal organisational structures are set (e.g. membership, executive, meetings, roles, and responsibilities). Trust and cluster identity are developed. The cluster engages in some initial projects that stimulate business collaboration between members (e.g. preparing a bid or developing the cluster's website). Members learn, share, and work together towards solving common problems.

In the implementation stage, clusters aim to win export contracts through the development of new products, service, or knowledge. Attention is focused on maintaining cluster momentum and on achieving the conditions for innovation and productivity within the cluster. New business alliances are formed inside the cluster, in the supply chain, and/or in export networks. New companies may be formed to address an international opportunity. The cluster begins to earn revenues and becomes partially self-sustaining.

In the improvement stage, the cluster develops innovative products and services. The environment is monitored to identify issues and market trends that may require the cluster to adjust its business practices, procedures, products, or services. The cluster's reputation grows and its brand strengthens in the target markets. Formal policies and procedures to manage resources, people, and relationships are put in place. The cluster is self-sustained through membership fees and project revenues and external funding is no longer required. The cluster is committed to reaching out for newcomers in order to broaden and deepen its network. Skilled labour and new business investment are attracted to the region as a result of the cluster's success. Finally, the cluster becomes a strategic partner for regional economic development initiatives.

For Lindsay (2005), the co-evolutionary approach provides a better understanding of the cluster development process. A key implication of this approach is the need for continual acquisition of external knowledge and its utilisation through the cluster. Both 
policy-makers and managers of firms within the cluster need to support and facilitate this to ensure the long-term viability of the cluster.

Unfortunately, clusters are not easy to form. Ceglie, Clara, and Dini (1999) provide three factors that obstruct the natural materialisation of clusters: 1) the high transaction cost of identifying appropriate network partners, as well as the cost of fostering these relationships; 2) the imperfect nature of the market environment, which complicates access to critical inputs for network development, such as innovation and information; and 3) the risk of "free riders" who want to benefit from the network or cluster, but are not planning to contribute towards the cluster.

To reduce the effects of these negative factors, the suggestion by Ceglie, et al. (1999) is to involve an "external agent" to facilitate the cluster and the network. Furthermore, Smedlung (2006) agrees on the relevance of these "intermediaries or intermediate organizations" (p.205) in enabling and supporting the transfer of knowledge inside the cluster. Usually this role is undertaken by central governments, local authorities, development agencies, or a combination of the three.

Cluster development has been more popular at the regional or sub-national level. Lagendijk and Charles (1999) give several reasons for clustering to be supported by local governments, including the fact that it contributes towards reducing the gap left by the "hands-off" position of central governments, as seen in the cases of regions in Germany, the United Kingdom, and the United States. They also suggest that local authorities found clustering a useful framework to attract foreign investment to their regions, by showcasing an environment with economic advantages for the foreign companies who base their operations there.

Lagendijk and Charles (1999) criticise the way some regions have developed a cluster strategy based on an analysis of the competitiveness of the existing economy and an identification of threats and opportunities in particular industries, usually dictated by the Porter approach of mapping clusters. They propose the alternative of networking and the creation of forums around clusters, which they believe is a bottom-up process where the policy maker's main task is to facilitate the networking process. In addition, they recommend the appointment of a regional development agency to commission research and consultation, facilitate the support frameworks for bottom-up clustering, and develop action plans. 


\subsubsection{The role of clusters in the Knowledge Economy}

A large body of the literature proposes that innovation is enhanced by the presence of clusters (Bell, 2005; Schiele, 2008). Innovation has been conceptualised by the OECD (1999:9) as "the ability to manage knowledge creatively in response to marketarticulated demands and other social needs." Innovation not only implies bringing something new to the market, it also entails the introduction of new processes and tools that improve existing products or services ahead of their competitors (Bentley, 1998; Simmie and Sennett, 1999). Malmberg and Power (2005) argue that innovation can be the result of tackling a problem, where a firm approaches a customer, a supplier, or even a competitor for assistance in defining the problem and/or reaching a solution that will result in industrial innovation and change. Innovation is triggered by gaining knowledge from personal experience, applying scientific research, or studying publications, patents, and blue-prints (OECD, 1998).

For Enright and Roberts (2001), clusters foster innovation because they provide a short feedback loop, they become the repositories for industry-specific skills and capabilities, they attract talented people, and they provide a focal point for investment and new business activities. In their empirical study, Cainelli and De Liso (2005) confirmed that clusters provide a favourable environment for firms to carry out product innovation. One aspect the authors were unable to identify was the source of such innovation - was it just internal to the cluster, from formal or informal agreements, or from collaboration with research institutions?

Maskell (2001) explains how new firms which have spun off from a cluster do tend to stay nearby to "safely skip the burdensome and costly process of gathering a lot of circumstantial knowledge about the business environment" (p. 933). These new firms therefore benefit from being surrounded by the appropriate conditions to increase their chances of survival and growth.

Governments and development agencies view clusters as a viable option for small- to medium-sized enterprises (SMEs) to raise their innovation capacities and competitiveness (Karev, Koh \& Szamosi, 2007; OECD, 1999). By clustering, SMEs can link together and overcome some of their limitations, such as their inability to capture market opportunities; their lack of training, market intelligence, logistics, and technology innovation; and their tendency to lock themselves into retaining slim profits (Bailey et al., 2002; Ceglie et al., 1999). 
Nonetheless, it is important to acknowledge that other authors argue that alternative inter-firm arrangements are as good as clusters in explaining innovation and growth in different kinds of businesses (Gordon \& McCann, 2005; Perry, 2007). For instance, Perry (2007) explored the perceptions of 46 enterprise managers in the New Zealand forestry industry about the usefulness of local (cluster) versus national (industry association) arrangements. His overall findings indicated that there was no clear preference for one form of association over the other; however, interactions with business members and business development were more favourable in the cluster arrangements, whereas liaison on standards and government regulation was best supported by industry associations.

After reviewing the empirical evidence of over 100 studies about clusters available at the time, Malmberg and Power (2005) concluded that, so far, clusters seem to serve as sites of informal social interaction and a space for specialised and skilled labour, rather than localised systems of tightly-bounded interrelated firms carrying out transactions and collaborations. For them, the role of clusters in innovation is a lot less than geographers and regional scientists might like it to be.

\subsection{Knowledge and its transfer in clusters}

Authors like Kluge et al. (2001) and Simmie (2003) argue that knowledge should be considered the fourth factor of production, along with labour, land, and capital. Knowledge did not get much attention in the past because the other three factors of production were abundant, accessible, and considered to be the reason for economic advantage. As tangible productive factors are currently no longer enough to sustain a firm's competitive advantage, knowledge is being called on to play a key role (Drucker, 1994; Lee \& Al-Hawamdeh, 2002; Simmie, 2003).

With knowledge becoming a valuable competitive advantage, it seems strange that many firms opt to partner with other companies and transfer knowledge. A closer examination of the situation reveals that many firms in the current competitive economic environment do not always posses the material, financial, or knowledge capacity to operate entirely on their own (Bryant, 2003). Knowledge is therefore created, exchanged, and even reused in such partnerships. 
Within a cluster, collaborative arrangements take place between various firms aiming to provide a novel service or product to the market. Pinch, Henry, Jenkins, and Tallman (2003) state that the specialised knowledge stock created and/or acquired by a cluster allows its members to enjoy competitive advantages over other firms in the same industry outside the cluster.

For knowledge to be an effective productive factor it must be current, easy to locate, and in the right form for organisations to benefit from it (Alavi \& Leidner, 2001). This section looks at the concept of knowledge and explains its role in the cluster environment. Then the concept of knowledge transfer is introduced. In particular, the activities of knowledge sharing and re-use are examined in the context of clusters.

\subsubsection{The concept of knowledge}

Despite being closely related, data, information, and knowledge are different concepts (Davenport \& Prusak, 1998; Keane \& Mason, 2006). The confusion between such terms has resulted in firms using unnecessary resources to develop technology projects to manage knowledge, which neither worked nor met expectations (Keane \& Mason, 2006; Sveiby, 1997). A description of the first two terms will follow, before focus turns to the concept of knowledge.

Data are discrete and objective facts about an event - e.g. a purchase transaction, a price, or the name of a company - that are represented in a symbolic manner, like in numbers or letters (Davenport and Prusak, 1998). Data on their own lack relevance or purpose because they cannot explain aspects about the event, such as why it happened or predict its outcome. Nevertheless, data are the raw material which organisations need to operate and to answer questions such as what, where, when, who, how big, or how many (Snyder, C. \& Wilson, L., 2000).

Information, on the other hand, is data that have been processed, and thus it has some added value that will provide insights for decision-making (Davenport \& Prusak, 1998; Drucker, 1998). Davenport and Prusak (1998) suggest that data can be transformed into information after one or several of these processes: a) contextualisation, by explaining the purpose of the data; b) categorisation, by defining data into units of analysis or key components; c) calculating, by analysing the data using mathematics or statistics; d) correction, by removing errors from data; and e) condensation, by summarising data to form a concise message. Therefore information has a meaning; it is relevant and has a 
purpose. However, it is up to the receiver to decide if the message has truly informed them; otherwise it is mere noise.

When data and information are assessed by one or various individuals using a combination of mental processes learned through experience and practice, it becomes knowledge (Bhatt, 2001). Nonaka, Toyama, and Konno (2000) add that knowledge is highly contextual, given its reliance on a specific place or time. Davenport and Prusak (1998) define knowledge as:

... a fluid mix of framed experience, values, contextual information, and expert insight that provides a framework for evaluating and incorporating new experiences and information. It originates and is applied in the minds of knowers (p. 5).

Davenport and Prusak (1998) identified four processes that transform information into knowledge: a) comparing the information of this instance with other known situations; b) determining the consequences to which such information may lead; c) connecting, by relating this new information to other information; and d) talking with other people to learn their opinion of this new piece of information. At least for the moment, these four processes can only be done by humans; no computer is yet smart enough to help.

Alvesson and Kärreman (2001) point out that knowledge is a vague concept because it has been applied to: facts (encyclopaedic), instructions on how to carry out an activity (procedural), determination of when is appropriate to use encyclopaedic or procedural knowledge (social), or determination of the reason why (explanatory). These authors warn that knowledge is not always objective, reliable, "functional, useful, and a generally good thing" (p. 999). Hence, knowledge users need to be aware of its potential limitations.

Knowledge has been viewed as either explicit or tacit (Singh, 2007), following the dichotomy proposed by Polanyi in the 1960s (Pinch et al., 2003). Explicit knowledge relates to a process or a product developed by experts that can easily be systematically codified. Explicit knowledge is available for sharing through books, manuals, documents, blueprints, patents, databases, and other software because this knowledge is rule-based and schematic (OECD, 1998; Simmie, 2003; Snyder \& Wilson, 2000).

On the contrary, tacit knowledge consists of "behavioural and social patterns, intuitive learning skills, beliefs, and perspectives" (OECD, 1998:198). Pinch et al. (2003) add that tacit knowledge is thought to come from hands-on experience and in-depth 
understanding developed through practice. Given these properties, tacit knowledge cannot be easily codified, thus making its distribution difficult. Devlin (2001) indicated that an effective way to transfer tacit knowledge is by observing and interacting with an expert at close range - such as in partnership, mentoring, or apprenticeship arrangements - in a process he called "knosmosis ${ }^{3}$."

Nevertheless, a more recent view on knowledge promotes "knowledge-as-continuum" (Jasimuddin, Klein \& Connell, 2005; Guzman \& Trivelato, 2008; Taylor, 2007). This view advocates that Polanyi's concept of knowledge has been misinterpreted (Keane \& Mason, 2006): rather than being two types of knowledge - that is, tacit and explicit knowledge has both tacit and explicit dimensions. Hence, a piece of knowledge would fall somewhere between the tacit and explicit poles of a continuum.

Another debate in the current knowledge management literature is the one put forward by Keane and Mason (2006), which states that the difference between information and knowledge should be relaxed. The authors are not proposing that these two terms be used synonymously - although some authors have done so, as pointed out by Iammarino and McCann (2006) - but rather recognise that "information may be viewed as the explicit representation of knowledge, which is composed of both tacit and explicit dimensions" (p.4). Their argument is based on the fact that, in order to make sense of a document, an individual will take contextual knowledge into account, such as the culturally shared and accumulated knowledge. Simmie (2003) provided the example of a circuit diagram, which is an explicit piece of knowledge that can be understood only by individuals with the right experience, learning skills, and intuition.

Knowledge is not a new idea (Kluge et al., 2001). Organisations have benefited from it, while philosophers and scholars have been studying it for centuries. Examples of firms focussing more on experience than on qualifications or intelligence when recruiting personnel, or people in the firm seeking advice from knowledgeable individuals when faced with difficult decisions, confirm that firms appreciate knowledge (Davenport \& Prusak, 1998). However, it was not until recently that this concept was explicitly considered a competitive advantage.

An attribute of knowledge pointed out in the literature is the fact that knowledge is a sustainable source of economic growth, as it does not deplete over time like the other

\footnotetext{
${ }^{3}$ Suggesting that tacit knowledge is acquired in a similar fashion as the biological process of osmosis, where cells absorb nutrients through their walls.
} 
factors of production (Romer, 1986 \& 1990; Simmie, 2003). This is because, unlike other goods and services, knowledge is not lost through sharing. On the contrary, knowledge generates more knowledge, as ideas created from present knowledge spark new avenues for exploration. Firms that are knowledge-rich, knowledge-oriented, and avid learners will have moved on by the time competitors have managed to emulate their initial level of quality, creativity, or efficiency.

\subsubsection{Knowledge in clusters}

The role of knowledge in creating competitive advantage has triggered the interest of scholars in understanding knowledge in the cluster's establishment and competitiveness (Santos, 2006), as well as the interaction between knowledge creation and this geographical economic system (Malmberg \& Power, 2005).

Maskell (2001) produced a conceptual framework explaining the creation of knowledge within clusters. He described the horizontal (rivals and competitors) and vertical (business partners and collaborators) dimensions of a cluster. The co-location of similar firms in the horizontal dimension creates knowledge when cluster members monitor, discuss, compare, imitate, learn, and add ideas of their own in a "focus and transparent environment" (p. 934) with low transaction costs. The input/output relationships in the vertical dimension demand that dispersed knowledge be brought together to assist with co-ordination and communication, resulting in a favourable environment in which to further develop specialised knowledge.

Maskell (2001) then highlighted that knowledge creation in these two dimensions is influenced by sets of institutions (formal and informal constraints that structure human interactions, such as rules, norms of behaviour, and codes of conduct) defined by the cluster over time. Molina and Yoong (2003) listed a few of these institutions while looking at the factors that influence knowledge sharing in clusters: a common understanding of the cluster idea, trust, and having "show and tell" meetings. Some of these institutions are generic and applicable to all clusters, while other institutions are specific responses to particular conditions faced by the cluster and thus determine "how learning takes place" (Maskell, 2001:934). These institutions will define the boundaries of the cluster's knowledge stock.

Clusters have been attributed the ability to absorb and use knowledge spillovers that would normally be wasted outside the firm (Dahl \& Pedersen, 2004; Iammarino \& 
McCann, 2006). Juniper and Metcalfe (2000:3) define spillovers as "social benefits flowing from innovation to third parties who do not participate in the basic contractual relationship that generates know-how, but gain benefits that cannot be defined, assigned clear property rights and thus, explicitly, traded in the market place."

However, Alsleben (2005) warns that setting-up policy promoting clustering in a region should not be solely based on the idea that clusters will absorb knowledge spillovers. Co-location brings other negative factors that individual firms should consider, such as labour poaching and "exposed" knowledge.

Cainelli and De Liso (2005) argue that clusters play a more active role than just being the passive recipients of unintentional knowledge spillovers. In the context of Italian industrial districts, they observe that the division of labour (i.e. organising the process of production), forms of learning, and the diffusion of knowledge within the group are activities of intentional nature that generate and use knowledge.

Lindsay (2005) and Maskell and Malmburg (2007) warned clusters about "inertial view" or "myopic behaviour," which has the potential to impair clusters from noticing new opportunities and acting on them. Inertia in the form of "inflexibility of managers' mental models, institutional pressures, and strong intra-cluster linkages" (Lindsay, 2005:77) could lead a cluster to focus on its internal dynamics and knowledge flows, and hence lose touch with the external context where new information, institutional patterns, and knowledge are constantly emerging.

For Lindsay (2005), clusters should subscribe to a "co-evolutionary view," where a cluster's long-term and sustainable performance is the result of cluster members' commitment to maintain and increase their external knowledge stock by coevolving with the cluster's environment at individual, company, community, and industry levels. Along these lines, Malmberg and Maskell (2007) encourage clusters to continually develop their absorptive capacity - that is, the ability to assimilate new knowledge and to build pipelines to external knowledge pools.

Although counting on a stock of knowledge is important, clusters gain competitive advantage only when members are able to effectively transfer their knowledge in order to innovate and to take action. Consequently, how a cluster manages to do this becomes crucial. The next sections will further explore the concept of knowledge transfer and how it fits within the context of clusters. 


\subsubsection{Knowledge transfer}

Argote and Ingram (2000) define knowledge transfer in the organisational context as “...the process through which one unit (e.g., individual, group, department, division) is affected by the experience of another" (p. 151). As Liu (2007) pointed out in his analysis of the current literature on knowledge transfer, the causes, consequences, and effects of this phenomenon has been extensively researched at various levels, including individual, project team, organisational, and inter-organisational. Authors like Albino, Garavelli, and Gorgoglione (2004), Easterby-Smith, Lyles, and Tsang (2008) and Minbaeva (2007) have used elements of communication models to explain knowledge transfer, where a sender transmits a message (knowledge) to a receiver within a particular context.

Of particular interest is the inter-organizational knowledge transfer framework proposed by Easterby-Smith et al. (2008), shown in Figure 4. Four elements are identified: donor firm, recipient firm, nature of knowledge, and inter-organizational dynamics. Each element has a set of factors that influence the effectiveness of the transfer of knowledge between the sender and the receiver.

\section{Inter-Organizational Knowledge Transfer}

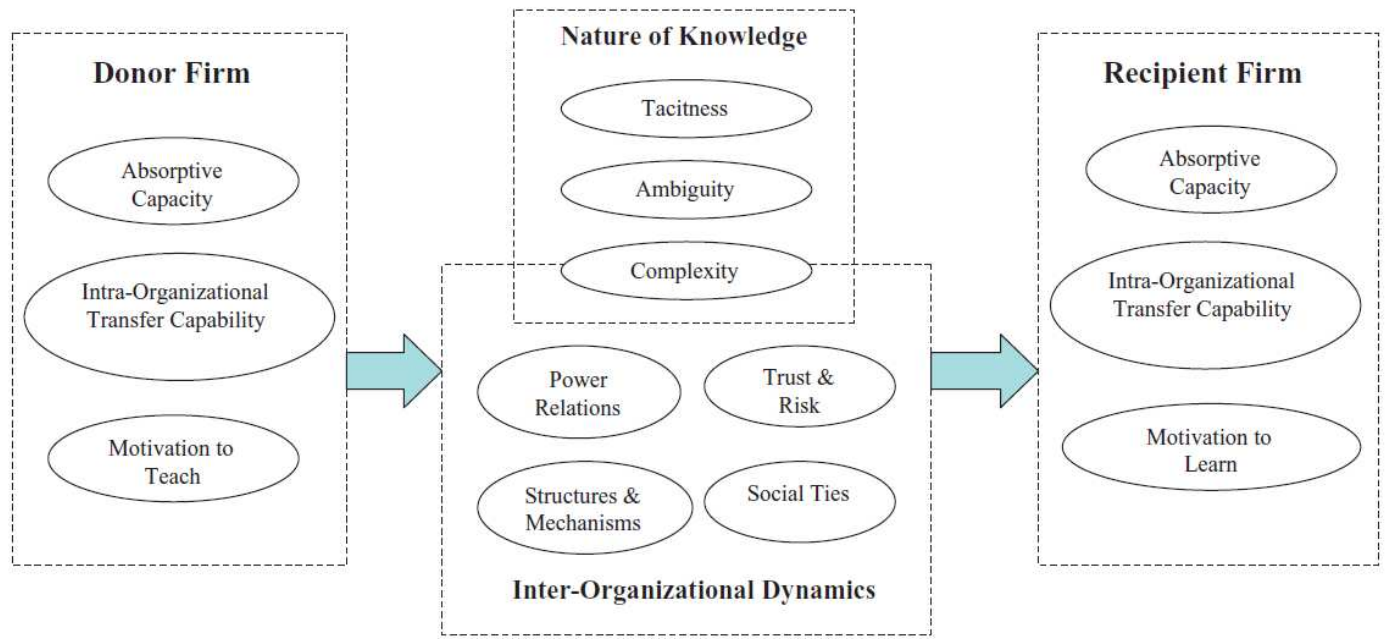

Figure 4. Factors influencing inter-organisational knowledge transfer (source: Easterby-Smith et al., 2008).

The factors to be considered for the donor and the recipient firms are their absorptive capacity, their respective intra-organizational transfer capacity, and their motivation to teach (for the donor) and learn (for the recipient). As mentioned in the previous section, absorptive capacity is the ability to recognise, assimilate, and use new knowledge. The 
absorptive capacity by the recipient will be determined by their basic skills, a shared language, and relevant prior experiences. The authors argue that firms also need to have intra-organisational capabilities to diffuse the acquired new knowledge within the organisation. The motivation to teach/learn will depend on the willingness, incentives, and norms that are in place to share knowledge.

For nature of knowledge, Easterby-Smith et al. (2008) identified three factors to consider: the degree of "tacitness," ambiguity, and level of complexity. This will impact on how the new knowledge can be articulated, how readily available it is to be used by the recipient, and what other associated routines, technologies, individuals, and resources would also need to be communicated to make sense of the particular knowledge (Minbaeva, 2007).

Finally, the effective knowledge transfer will depend on how the inter-organizational dynamics - in other words, the context - are played between the donor and recipient, which Easternby-Smith et al. (2008) have grouped in four factors: power relations, trust and risk, structures and mechanisms, and social ties. There will be an asymmetry of power between the donor and the recipient of knowledge which could affect the bargaining power of one party over the other, and with time this could deteriorate the basis of their cooperation. There is risk for the donor that some knowledge is unintentionally transferred that could erode its competitive advantage, and for the recipient that the knowledge given is not useful; the level of trust between the parties minimises these issues and ensures that the transferred knowledge will be only used for its initially intended purpose. Structures and mechanisms are established to facilitate the knowledge transfer. In addition to formal structures put in place, informal social ties between both parties also channel knowledge transfer.

Guzman and Trivelato (2008) studied the transfer of codified-oriented knowledge, which they considered the "most taken for granted aspect of knowledge transfer" (p.251). For them, codified-oriented knowledge is "knowledge that, although it is, or can be codified to a significant extent, still needs tacit knowledge in order to be codified, transferred, stored, understood, and distributed" (p.254). The authors propose that codified-oriented knowledge takes a three-stage process: knowledge codification, which involves extracting the knowledge from the individual and transform it into generic knowledge that is written up into some sort of manuals or blue-prints; mechanisms used to transfer knowledge, which will depend on the organization, the 
stages of knowledge management, and the knowledge's degrees of tacitness; and knowledge assimilation and application, which entail decodifying, understanding, and interpreting the knowledge.

\subsubsection{Two aspects of knowledge transfer: sharing and reuse}

Majchrzak, Cooper, and Neece (2004) argue that knowledge transfer entails two key aspects: knowledge sharing, in which new knowledge is circulated; and knowledge reuse, in which existing knowledge is considered in new situations. The following subsections will discuss these concepts in more detail.

\subsubsection{Knowledge sharing}

For Appleyard (1996:3) knowledge sharing is "the transfer of useful know-how or information across company lines." A feature of knowledge sharing mentioned by Connelly and Kelloway (2003) is the fact that it is reciprocal; in other words, the involved parties usually gain something from the experience - more knowledge, perhaps. Knowledge sharing takes place through apprenticeships, networking, team working, informal gossip, and facilitation (TFPL, 1999).

Ciborra and Andreu (2001) describe three main situations where knowledge sharing takes place: within a single organisation, among a small number of organisations in a strategic alliance arrangement, and within a web arrangement of multiple organisations.

\section{a) Within a single organisation}

In this scenario, the firm attempts to reach competitive advantage by searching for better work practices to exploit their current resources (or, alternatively, introducing new resources to make them look for better work practices). Firms are encouraged to provide spaces for the effective mixing of internal and external knowledge, as well as capturing the knowledge generated and sharing it with the rest of the organisation. Intra-firm knowledge sharing has been extensively studied in the literature (Connelly \& Kelloway, 2003; Han \& Anantatmula, 2007; Vera-Muñoz, Ho \& Chow, 2006).

\section{b) Among a small number of organisations in a strategic alliance}

Gulati (1998:293) defines strategic alliances as "voluntary arrangements between firms involving exchange, sharing, or co-development of products, technologies, or services." Alliances occur among companies, regardless of their differences in size 
and strength, in order to achieve a common goal that a single organisation cannot accomplish (Berquist et al., 1995).

Due to the small number of participating organisations in the alliance arrangement, there is a focus on how compatible and transferable each firm's practices, routines, and capabilities are. This is necessary as there will be a recombination of approaches to develop new distinctive knowledge, routines, and competencies that will be used by the partnership (Ciborra \& Andreu, 2001). There must be governance in the alliance to guarantee coordination, transferability, and value generation between partners.

\section{c) Within a web of multiple organisations}

In this arrangement, multiple knowledge-intensive organisations gather together around a core technology or competency and establish informal networks to develop new products or services. Ciborra and Andreu (2001) argue that it becomes difficult to distinguish the constitution of resources, practices, routines, and capabilities provided by the participating organisations in the web setting. For these authors, industrial districts (i.e. clusters) are an example of firms interacting in a web arrangement context.

Despite the fact that knowledge is a valuable factor of production and companies would rather keep it for themselves as a competitive advantage, there are theoretical and empirical studies indicating the opposite. Technical change and innovation in a wide range of industries, such as electronic telecommunications, semi-conductors, and biomedicine, can be achieved only if firms have access to external sources of knowledge and interactive learning (Appleyard, 1996; Bryant, 2003; Chaminade, 1999; Ciborra \& Andreu, 2001). Thus, firms that are actively interested in the process of innovation and knowledge creation engage in nurturing external links with other firms, universities, and public and co-operative research agencies (OECD, 1998; Snyder \& Wilson, 2000; Pöyhönen \& Smedlund, 2004).

Companies decide whether or not to share knowledge with other firms depending on the anticipated costs and benefits of doing so (Lee \& Al-Hawamdeh, 2002; Loebbecke et al., 1999). Issues on how to manage this process, such as the extent and nature of the knowledge, and when, with whom, and under what circumstances the knowledge is disseminated, must be taken into account by all parties (Loebbecke et.al, 1999). 
Knowledge sharing is common among firms that are part of a value chain (Chaminade, 1999; Kluge et al., 2001; Snyder \& Wilson, 2000). Firms that work closely with their suppliers can gain knowledge in production processes and procedures that will help to improve efficiencies. By exchanging ideas with distributors, retailers, and end-users, a firm can learn about marketing, product development, and design.

In the cluster context, Molina and Yoong (2003) looked at the factors that influence knowledge sharing among members of business clusters based in Wellington, New Zealand. The authors focused their study on two of the four stages of the cluster lifecycle ${ }^{4}$ : initiation and incubation. Their findings outlined the conditions that favour knowledge sharing practices, such as having a common understanding and commitment to the cluster idea, a high level of trust, and strong leadership. Nevertheless their study did not elaborate on the type of knowledge, how knowledge sharing was being carried out, or what mechanisms were being employed by the clusters.

Mei and Nie (2007) explored the innovation impact of knowledge sharing when firms interact with their customers and suppliers in the optoelectronic cluster of Wuhan, China. The authors focused on exploring two of the knowledge transfer barriers, absorptive capacity and the knowledge characteristics, and how they impact knowledge sharing and consequently influence innovation. Their study suggested that, although a high degree of knowledge sharing between a firm and their customers and suppliers produces more innovation, the firm's absorptive capacity plays a more important role. How well a firm assimilates the shared knowledge is more important than the knowledge characteristics. It is worth noting that this study only focused on relationships in the value chain; in other words, the authors only looked at the cluster's vertical dimension of Maskell's conceptual framework.

\subsubsection{Knowledge reuse}

Knowledge reuse is described by Markus (2001) as a process encompassing the stages of capturing, packaging, distributing or disseminating, and reusing knowledge. Capturing knowledge can be done as: a by-product of a work process, such as files generated from communications that took place while working on a task or a project, which can be searched and accessed later on; a structured action such as brainstorming techniques, which attempts to capture knowledge for potential use in the future; a deliberate pre-determined format to collect knowledge "before-the-fact;" and a review 
or learning histories, a product of an "after-the-fact" assessment of the situation. Packaging involves preparing the captured knowledge to make it consumable in the future, such as authoring, filtering, and pruning knowledge content. Distribution could be passively achieved through newsletters or databases, or actively accomplished through meetings and electronic alerts. Finally, reusing involves recalling the collected knowledge and applying it to a new context.

Owen, Burstein, and Mitchell (2004) indicate that if a firm wants to reuse knowledge effectively it needs to acknowledge the value of its organisational memory. For the authors, organisational memory is not a central knowledge repository, but rather the knowledge found in individuals, the organisation's culture, transformations, structures, ecology, and external archives. Similarly, Markus (2001) views knowledge reuse as both "access to experts and access to expertise" (p. 58).

Haefliger, von Krogh, and Spaeth (2008) look at knowledge and code reuse (i.e. algorithms and methods, line of code, and components) for software development. They observe that success in code reuse in software development firms will depend more on organisational factors than on technical ones. The firm needs to fund repositories and libraries, and provide tools and standards for developers to search for code. Champions, sponsors, and re-use coordinators need to be appointed to facilitate knowledge reuse. Finally, an incentive programme needs to be in place for developers to overcome the "not-invented-here" syndrome or the idea that re-using code is "boring." The authors compared the practices of knowledge and code reuse in open source informal communities and found that similar strategies to commercial firms were in place, despite the fact that there are no financial resources available to cover tools, standards, or incentives.

Majchrzak et al. (2004), Newell (2004), Newell, Bresnen, Edelman, Scarbrough, and Swan (2006), and Owen et al. (2004) explore knowledge reuse at project level in various domains (banking, healthcare, automotive, bioscience, engineering, and consulting). Newell (2004) mentions that organisations aware of the importance of recording the lessons learned on a project have subscribed - at least in principle - to prepare project reviews at the end of a project. These are then stored in electronic databases for other teams to access via the company's intranet by typing in the project title or keywords. However, Newell (2004) observed that, in practice, people are quickly

\footnotetext{
${ }^{4}$ The cluster development life-cycle model was explained in section 2.2.4.
} 
allocated to other project teams, thus there is no time to conduct a thorough review of the project. She also noticed in her four case studies that such codified end-of-project learning reviews were seldom reused; people relied instead on their social networks.

Newell (2004) believes the problem is two-fold. Firstly, initiatives for cross-project learning tend to ignore the needs of the receiving audience; consequently, supply does not meet demand. Secondly, teams tend to believe that each project is unique and therefore do not attempt to review the available knowledge.

This is why Newell (2004) suggests a change in the focus of project reviews, from what has been done to a reflection on how it was done; in other words, a shift from "product knowledge" to "procedural knowledge" (p. 18). This will make the lessons learnt relevant to new projects. At the same time, it is important to make the audience more receptive. Teams must be persuaded to see beyond the uniqueness of a project and learn from the experience of others. Finally, Newell (2004) considers that social networks are "a necessary compliment" (p. 19) to databases, intranets, and any other ICT system in place for knowledge transfer.

Based on the findings of their empirical study, Newell et al. (2006) determined that, in practice, project teams relied more on social networks for cross-project knowledge transfer than the use of ICT solutions such as databases and intranets. This was due to the fact that the knowledge found in these ICT systems continue to be more about what was done in a project, instead of how the team carried out the project or the lessons they learnt along the way - which will be more relevant to future projects. This highlights once again the fact that knowledge reuse is not a technology issue, but rather an organisational one.

Markus (2001) also notices the problem of knowledge reusers not always getting the appropriate knowledge. She argues that a reason for this is because knowledge producers are unaware of the needs of other knowledge reusers. Markus (2001) proposes a typology of four knowledge reusers, based on the type and purpose of the knowledge: shared knowledge producers, shared work practitioners, expertise-seeking novices, and secondary knowledge miners. Each reuser would demand attention to particular issues to ensure successful knowledge reuse.

Shared work producers create and document knowledge that they may use later on, such as keeping track of the status of the project, and records of decisions made and actions 
taken. They usually form part of a team of people in the same field (e.g. software developers) or a multi-disciplinary team working towards a common purpose (e.g. product development). Since shared work producers are the consumers of their own knowledge, they tend to reuse "raw" knowledge that may seem "incorrect, incomplete, or incoherent" (Markus, 2001:68). This because they are aware of the context, the implicit knowledge, and the assumptions made when this "raw" knowledge was created.

Shared work producers assume that nobody else apart from themselves and their team would review this knowledge, and therefore tend to produce sketchy notes, have bad filing systems, and even forget what they have already documented. Markus (2001) recommends the use of a knowledge intermediary to assist this group in the indexing, location, and retrieval of knowledge. In addition, the team has to not only document what they are doing, but also the rationale behind their decisions.

Shared work practitioners are people working on similar roles but in different locations, work units, and even organisations, who gather to form a community of practice and "produce knowledge for each other to use" (Markus, 2001:68). The community has a general understanding of its common domain, but the lack of contextual knowledge of a particular instance could make knowledge reuse difficult. This is why shared work practitioners rely on their network of contacts to find the appropriate document or expert who can assist them. Therefore, clear authorship of the documentation, indication of the recentness of the knowledge, proper indexing, access to experts, and the establishment of incentives for contributions and reuse, are some considerations suggested by Markus (2001) to ensure knowledge reuse by this group.

The expertise-seeking novices are occasional consumers of knowledge produced by experts. As the name indicates, the expertise-seeking novice is relatively new to a particular domain of knowledge, and uses the expertise of others to answer a query rather than acquiring such knowledge themselves. Given their neophyte condition in the field, this group may find it difficult to articulate the right questions and use the proper jargon to navigate their way through knowledge. For this reason, Markus (2001) points out that knowledge has to be repackaged to eliminate excess of context (i.e. "decontextualisation") that would otherwise confuse the expertise-seeking novice. The use of a knowledge intermediary to assist in locating and assessing the quality of the documented expertise and the experts, as well as helping relate that knowledge to particular settings, would be highly advantageous to this group. 
The last group of knowledge reusers are the secondary knowledge miners. This group is also a consumer of knowledge produced by other people whom they do not know. Secondary knowledge miners are usually expert analysts developing new knowledge by reusing knowledge that was originally created for other purposes. To improve knowledge reuse in this situation, Markus (2001) proposes recording context information or metadata in the knowledge repositories to facilitate secondary reuse as well as providing pertinent training for these experts in analysis and synthesis.

From these descriptions, Markus (2001) points out two general recommendations for successful knowledge reuse. The first is to design proper incentives for contributing and using repositories. Some examples Markus (2001) mentions are: enhanced reputation among colleagues, a repository that helps individuals do their jobs, and knowledge production included as part of the evaluation criteria for promotions and bonuses. The second recommendation is to allocate some of the knowledge packaging and knowledge dissemination responsibilities to intermediaries. An intermediary "prepares knowledge for reuse by eliciting it, indexing it, summarizing it, sanitizing it, packaging it, and . . performs various roles in dissemination and facilitation” (Markus, 2001:61). Although much of the intermediary work is done by humans, information technology is playing an increasingly important role in supporting this function.

Newell et al. (2006) agree that intermediaries can play a key role in ensuring the knowledge acquired or learnt by one part of the organisation can be employed by another team. In their case studies where intermediaries were overseeing several projects, these individuals were effective in encouraging knowledge reuse by promoting teams to learn from the experience of others. However, the intermediaries tended to connect people with other people rather than connecting people with documents.

Knowledge reuse in the context of clusters has not been widely explored in the literature. Clusters have the potential to generate knowledge to satisfy the needs of Markus's (2001) types of knowledge reusers. Within a cluster, teams formed by people from various organisations tackle business opportunities or projects. During the lifespan of the team, knowledge about contacts, processes, facts, and the experience itself is created; such knowledge could be reused by the team later on or even in future projects with similar characteristics. The lessons learnt and documentation generated in each project could be reused to speed up or improve the quality of the cluster's business processes, products, and services. 
Clusters are formed by companies with interests in a common domain, ${ }^{5}$ which have a similar technical knowledge and business drive; these companies understand the context and meaning of the knowledge created within the cluster. In other words, a cluster can be considered a community (Tallman et al., 2004). The knowledge and lessons learnt by members working on a project could be shared and reused by the rest of the cluster community. Furthermore, people supporting these clusters, such as the cluster facilitators, could also themselves form communities. Cluster facilitators perform similar roles in different clusters and could benefit from exchanging experiences and learning from each other as a community.

Expertise-seeking novices and secondary knowledge miners in the cluster context could be people working for economic development agencies, policy makers, journalists, and researchers. Some of these players will occasionally visit the clusters to enquire about a particular issue, but do not need an in-depth knowledge of the cluster concepts or its context. Researchers may be particularly interested in analysing the knowledge produced by clusters to identify patterns and trends, or to generate new knowledge about clusters.

\subsubsection{Knowledge transfer mechanisms}

The literature shows that knowledge transfer - for both sharing and reuse - is a decision that individuals and organisations have to make. As already pointed out, there are behavioural, financial, organisational, and contextual considerations to address before this process occurs. This study is interested in understanding how transfer takes place once the organisations in a cluster have agreed they will exchange knowledge with the rest of the cluster.Therefore, this section will explore further what the literature says about the mechanisms that are used for knowledge transfer.

Although Appleyard (1996), Brandt-Husman (2001), and Lee and Al-Hawamdeh (2002) were referring to knowledge sharing mechanisms when defining them as the means through which knowledge will be passed on to partners, this definition can be expanded to cover knowledge transfer mechanisms in general. Smeds, Olivari, and Corso (2001) were also talking about this same concept when describing the "vehicles to embody and transfer the gathered knowledge from one project to the next" (p.375).

\footnotetext{
${ }^{5}$ One example is the Earthquake Engineering New Zealand cluster based in Wellington. This cluster is comprised of engineers and scientists in earthquake protection engineering for infrastructure such as buildings, bridges, and roads.
} 
Appleyard (1996) categorises these mechanisms according to their access to (public or private) and use of (restricted or unrestricted) knowledge. She also provides examples of knowledge sharing mechanisms for each type (see Figure 5).

\section{Use of knowledge}

\begin{tabular}{|c|c|c|}
\hline & Restricted & Unrestricted \\
\hline$\frac{0}{0}$ & $\begin{array}{l}\text { - Review patents } \\
\text { - Reverse engineering } \\
\text { - Patented technology }\end{array}$ & $\begin{array}{l}\text { - Newsletter } \\
\text { - Popular press } \\
\text { - Trade journals } \\
\text { - Conferences }\end{array}$ \\
\hline$\frac{\vec{e}}{\tilde{s}}$ & $\begin{array}{l}\text { - Visit other companies' } \\
\text { fabrication plants } \\
\text { - Consortium } \\
\text { - Benchmarking studies }\end{array}$ & $\begin{array}{l}\text { - Email } \\
\text { - Telephone } \\
\text { - Face-to-face meetings }\end{array}$ \\
\hline
\end{tabular}

Figure 5. Knowledge sharing mechanisms (source: Appleyard, 1996).

Brandt-Husman (2001) and Jasimuddin (2007) indicate the importance of having an appropriate match between the knowledge transfer mechanisms and the nature of the knowledge that is to be shared, in order to keep transaction costs down and to guarantee effective transfer of knowledge. For instance, complex knowledge with a high tacit component should employ more personal knowledge-sharing mechanisms than those required by highly codified knowledge.

Jasimuddin (2007) explores the appropriateness of knowledge transfer mechanisms in a multinational corporation leader in computer manufacture and software development. He proposes that the appropriateness of a mechanism would depend on variables, such as status (the relationship in the organizational hierarchy), personal ties, and location (physical distance). Based on his case study, he developed the decision tree of media use for different transfer situations depicted in Figure 6. 


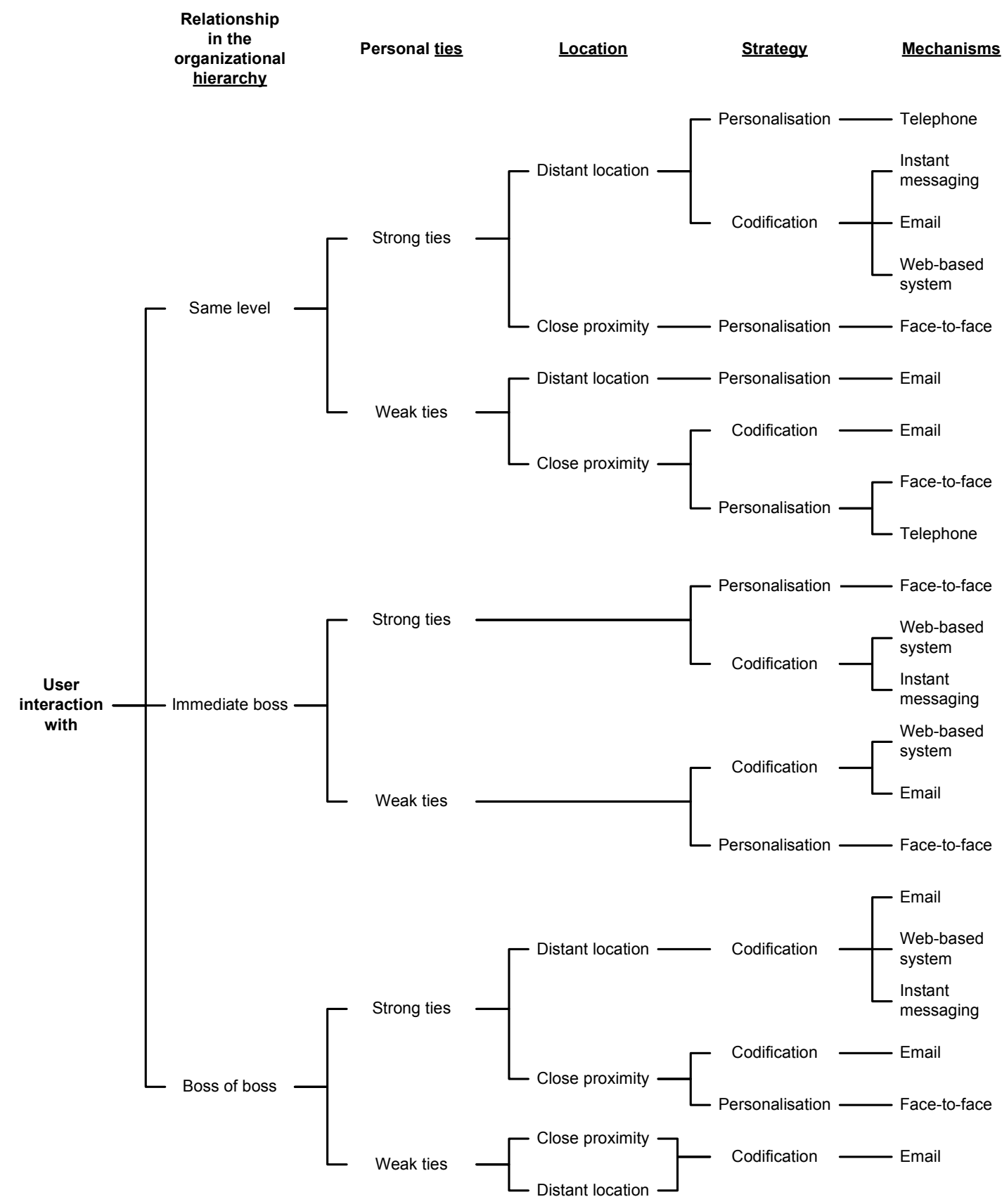

Figure 6. Decision tree to explore the appropriate knowledge transfer mechanism (source: Jasimuddin, 2007).

According to Jasimuddin's (2007) findings, people with the same status in the organisation would tend to engage in personalised mechanisms such as face-to-face or telephone. When there were strong personal ties, individuals comfortably employed any mechanism to transfer knowledge, whereas with weak personal ties they would tend to use technology-focused mechanisms, such as e-mail. When individuals were located closely to each other they were likely to opt for face-to-face interactions, and when they were separated by distance other media would be used, such as e-mail or any other 
technology-focus means. Jasimuddin (2007) recognized that further research should consider other variables that might also have a significant influence in knowledge transfer, like urgency, nature of the query, and trust.

Jasimuddin's (2007) concept of knowledge transfer mechanisms, however, appears to be synonymous of communication channels. It is my view that a knowledge transfer mechanism is something more than the communication medium being employed. Furthermore, Guzman and Trivelato (2008) argue that knowledge mechanisms cannot be generalised, as they are the result of the organisation's context, its knowledge management practices, and the degree of tacitness of the specific piece of knowledge to be transferred.

Acknowledging that given knowledge transfer mechanisms are closely related to the characteristics of the communication medium - but are not the same - Smeds et al. (2001) propose to categorise them into three groups: people, such as job rotation, meetings, forums, and face-to-face interactions; documents and databases, such as reports, meeting minutes, project documentation, project web pages, and product libraries; and organisational and technical elements, such as processes, procedures, rules, design tools, and components. Each organisation will have its specific portfolio of knowledge transfer mechanisms.

Smeds et al. (2001) identified and classified the specific knowledge transfer mechanisms employed by two R\&D units (one in Finland and the other in Italy) from a multi-national organisation, as their starting point to compare inter-project learning. Their findings show that the mechanisms under people transfer knowledge that is embedded in individuals, and can only be accessed through observing their actions and behaviours, or knowledge that can be externalised and communicated via dialogue, such as in conversations and meetings. The mechanisms under reports and database transfer knowledge that is highly codified, where human-to-human interaction is not required and knowledge is transmitted through physical or electronic mechanisms. Finally, those under organizational elements suit both knowledge that can be communicated via dialogue or that is codified, while the technical elements were entirely codified knowledge. 


\subsection{Information and Communication Technologies (ICT)}

Information and communication technologies (ICT), as part of the technological change in today's business environment, play a key role in the knowledge-based economies (Bergquist et al., 1995; OECD, 1998). ICT has supported changes in the production methods, consumption patterns, and economic structures in many countries (OECD, 1998). Monitoring suppliers, modelling new products, and communicating with partners are some of the activities facilitated by ICT that Scott (2000) enumerates in her work.

By the late 1970s and early 1980s, computing technology underwent a fundamental change when its hierarchical structure - comprised of a central mainframe, minicomputers, personal computers, and low-level "dumb" terminals - started to be replaced by a "distributed" or "network" approach (Bergquist et al., 1995; Turban et al. 2001). A new computing environment was emerging, characterised by potent desktop workstations that were interconnected among themselves as well as with high-powered information servers through telecommunication networks (Berquist et al. 1995; Jonscher, 1994).

Jonscher (1994) called this new computing environment the integration phase of information technology. ICT enabled organisations not only to merge informationhandling activities with their production and distribution activities, but also allowed them to link up with other organisations. Through the interconnection of their networks with external entities - e.g. suppliers and customers - firms reduced transaction and coordination costs, lowered inventory levels, improved their production schedules, and became more service-oriented (Jonscher, 1994).

ICT is also reshaping the strategies regarding inter-firm collaboration initiatives. In the Knowledge Economy, firms acknowledge that they belong to a larger system of suppliers, buyers, intermediaries, and competitors (Porter, 1990, Sveiby, 1997; Venkatraman, 1991). Given their nature, inter-firm arrangements usually have their knowledge distributed amongst the participating members and therefore managing knowledge becomes an important - and also challenging - task. Progress in computing power, together with price reductions in these technologies, are making knowledge transfer a feasible exercise (Lee \& Al-Hawamdeh, 2002).

The following sections will examine ICT's role in knowledge transfer and how ICT is being used to support knowledge transfer in the cluster environment. 


\subsubsection{The role of ICT in knowledge transfer}

ICT can support knowledge transfer in several areas: communication, co-ordination, location/storage, and collaboration. ICT can support the exchange of knowledge by providing appropriate communication infrastructure in the organisation - such as videoconferencing facilities, telephone lines, and groupware systems - so people can interact with others as if they were doing it in face-to-face situations (Davenport \& Prusak, 1998; Zhang, 2007). Web-based intranets also broaden the organisation's communication reach, by facilitating contact between individuals seeking knowledge and those who possess it, through electronic bulletin boards, discussion groups, and corporate directories (Zhang, 2007).

Nevertheless, some communication technologies still face difficulties in their performance. Delays in video or sound conferencing applications may affect the understanding of what is being communicated, as well as limiting social cues (Bryant, 2003). These issues are being addressed by the ICT industry and newer versions of such applications will improve their capacity to handle and transfer information.

ICT also assists in the co-ordination of activities between partners. Co-ordination was defined by Kumar and van Dissel (1996) as the process of executing agreed activities by a group of interdependent units through following pre-established protocols, tasks, and decision mechanisms. For instance, ICT can be combined with product design, manufacturing, and transportation technologies to coordinate supply and demand to reduce inventory in a value chain (El Sawy et al., 1999; Yates \& Benjamin, 1991).

ICT can provide the infrastructure to locate and store knowledge. Electronic libraries, portals, and peer-to-peer sharing networks are examples of these technologies (Zhang, 2007). Majchrzak, Rice, King, Malhotra and Ba (2000) indicate that being able to put knowledge in repositories capable of organising, retaining, maintaining, searching, and retrieving information is both an important starting point for knowledge sharing and the foundation for organisational memory.

Organisations are designing and implementing repository applications such as databases to store structured explicit knowledge about their products, markets, customers, and other relevant topics. Repositories based on World Wide Web technology are becoming popular due to their ease of use, their capability to display information across computer platforms, their multimedia features, and their ability to interconnect one piece of knowledge with others through hypertext link capability (Davenport \& Prusak, 1998). 
Knowledge that is highly tacit and knowledge that is highly explicit demand different approaches for their transfer. Davenport's and Prusak's (1998) rule of thumb is that the more tacit the knowledge, the more ICT should focus on linking people, rather than coding and storing it in repositories. For instance, knowledge maps pointing out who has knowledge on a particular issue and how to contact them would be better than keeping records. Albino et al. (2004) noted that organisations are constantly aiming at striking a balance between technology and human-centred approaches.

Communication systems are particularly valuable when the knowledge to be exchanged has a high tacit component given its unstructured nature and codifying difficulties. Explicit knowledge, on the other hand, has been ICT's traditional focus since it can be easily collected, organised, and transferred through digital channels (Pearlson \& Saunders, 2004).

ICT has reached the point where the technical aspect of enabling access to information and knowledge from anywhere in the world is no longer the main challenge to overcome. Instead, political and managerial issues with regard to organisational culture, structure, and strategy (Coakes, 2006; Zhang, 2007), which determine who will have access and control as well as how information and knowledge are going to be made available, have taken priority (Yates \& Benjamin, 1991; Turban et al., 2001; Li \& Williams, 2001).

\subsubsection{ICT supporting knowledge transfer in clusters}

Some studies in the cluster literature briefly mention the importance of emerging technologies - such as the Internet and workflow systems - in improving and accelerating inter-firm relationships, and thus making clusters more effective and efficient (Rosenfeld, 1997). DeFillipi (2002) indicates that ICT can support the network activity in clusters by creating common communication and collaboration spaces for business partners, while de Berranger and Meldrum (2000) explain how the Internet and the World Wide Web enable clusters to have a "brand" and global presence.

Carbonara (2005) reports the results of a survey on the implementation of ICT carried out on Italian industrial districts in the early 2000s. The survey indicated that the adoption of ICT-based interactions, such as mailing lists, discussion forums and videoconferencing, remained low; similarly low was the diffusion of advanced ICT solutions, such as shared databases or software for inter-organisational process 
integration. However, the survey showed an increase in portals and websites aiming at providing a virtual space to support collaboration within the district, promoting the district on a global scale, and even online trading (e-commerce) functionality. Carbonara (2005) argues that the low uptake of ICT is due to the significant investment needed for these technologies, as well as the business process re-engineering that the companies within the cluster would have to undergo.

Overall, there are few studies in the literature that explore in detail how ICT has been used to support cluster initiatives, and far fewer detailing how ICT enables knowledge transfer in business clusters. One such study is the work by de Berranger and Meldrum (2000). They give an account of a pilot project in the Northern Quarter - Manchester's creative sector - where ICT applications like electronic mail, the Internet, and the World Wide Web were introduced to the creative micro-business cluster. Participants in the project received training on these applications, and at the same time the Northern Quarter Network website was developed (www.nqn.org.uk, then shifted to www.mmu.ac.uk/h-ss/mipc/nqn/).

The study concluded that ICT brought the local businesses of the Northern Quarter closer together by engaging them in the development and training processes. The project also showed that the Internet has the potential to become a marketing tool for clusters of small companies to reach the rest of the world, where they can showcase themselves through a unified brand. Unfortunately this project lost momentum when the office and administrators were no longer available at the end of the pilot programme. As a consequence, the website became an information site for the region rather than a virtual network for the participants.

Quandt (1999) proposes the setting up of electronic networks to support firms that want to cluster but are not geographically close to one another, as a potential use of ICT in clusters. This concept was also explored in Braun's (2003a) doctoral study, which discusses how a dispersed tourism small-medium enterprise group in regional Victoria, Australia formed a virtual cluster. The study indicated that relationships were very complex and, although virtual networking has potential, ICT is still something new for many SMEs and appropriate infrastructure and technical support is paramount.

Braun continued this line of research and examined the adoption and diffusion process that occurred when the regional tourism network decided to introduce ICT and ecommerce for their sales and marketing activities. Braun (2003b) comments that the 
literature has focused on ICT adoption at individual SME level, but little has been covered on this subject in a network context. She also observes that the barriers affecting ICT adoption at network level derive from the internal dynamics and context of the network, such as the geographic make-up of the network, lack of convergent goals, individual status and position in the network, and the level of trust, worldview, and commitment of the individuals.

The small number of examples in the literature illustrating the role of ICT in supporting clusters does not mean that ICT is not actively involved in cluster development and knowledge transfer activities. For instance, Competitiveness (www.competitiveness.com), a consulting firm specialising in the design and implementation of clusters, offers the service of setting up ICT tools for clusters. In 2004, their website showcased two cluster portals they developed which are now the property of the ceramic cluster in Sassuolo, Italy and the textile cluster in Igualada, Spain. The textileclusters.com portal is an inter-firm tool allowing members to electronically communicate with their suppliers and customers without the need to install additional software.

Steinfield (2002), however, warns that people underestimate the location and proximity of geographically defined clusters when considering inter-organisational systems for these groups. Many systems - such as e-commerce portals - focus on reducing transaction costs, something that the cluster has already achieved by working together; consequently, any developed systems tend to be underutilised. Steinfield (2002) proposes that collaborative e-commerce initiatives for clusters should be designed to complement the rich communication and pre-existing relationships.

\subsection{Formulating the study's research questions}

Chapter 2 has provided a summary and synthesis of the literature in the topics of business environment, clusters, knowledge, knowledge transfer, and ICT. Besides introducing the main concepts of this study and examining their state of current knowledge and theoretical debate, the literature review has guided me in formulating the two research questions. This section brings the main concepts together, states my position regarding some of the views found in the literature, and identifies the gap in knowledge that triggered this study. 
The Knowledge Economy paradigm and the business environment are the background of this study. The literature review has established that firms are challenged to redefine their competitiveness as their business environment is constantly changing through technology advancements, factors endowments, and globalisation. Competitive advantage involves firms taking an innovative view of how to deploy new materials, procedures, and technology to bring superior products and services to the market.

At the same time, firms are aware that their competitiveness is being affected by what is happening outside their physical boundaries. Firms no longer succeed entirely by themselves. Those companies that understand the need for less accumulation and control of resources, and more interaction with other organisations to produce what the markets want, will successfully adapt to tackle these business challenges.

One organisational arrangement in this economic context is the cluster, which is the subject unit of this study. Defining a cluster remains a debate in the literature. Many terms have been used interchangeably with cluster (e.g. agglomeration, business networks, industrial districts, industrial clusters, and regional clusters), some authors argue that each term means a different thing, and others even try to avoid the debate altogether. It seems to me that employing one term instead of another is based on a particular feature of the phenomenon that is being emphasised, although at times the choice of term will depend on the researcher's background (Porterian or Marshallian), or the common usage in the country where the researcher is based.

Geographical, historical, and institutional factors, and change agents - or a combination of the four - can initiate cluster development. Reasons for clustering include opportunities to access new and complementary technologies, achieve economies of scope and scale, spread risks among members, combine complementary assets and knowledge, and accelerate the learning process. Perhaps because of the facts that clusters are not a "homogenous bunch," and they vary in size, depth, configuration, reasons for clustering, and stage of development, it has been difficult for the literature to provide a cluster definition that will include all of them.

Although clusters have been heralded as drivers of innovation and growth in a region, this cannot be generalised. The literature provides evidence supporting (such as the study by Cainelli and De Liso (2005) of the Italian-Marshallian industrial districts) or refuting (such as the econometric analysis by Gordon and McCann (2005) demonstrating that clusters in the London region did not show advantages for 
innovation) this argument. This leads to the conclusion that clusters should be assessed on a case-by-case basis, and the questions to ask are: under which conditions can a cluster be innovative, and what kind of innovations can be derived from clustering.

This study acknowledges the recent views and debate on the concept of cluster and the importance of further exploring its role in innovation and economic growth in a region. However these are themes that are better researched by people in the fields of economic geography, political science, or international business. Given that this study is embedded in the discipline of information systems, my interest in looking at clusters as the research subject unit lies in its attribute of being a space where inter-firm knowledge flow takes place, with a further interest in shedding light on the role of ICT.

Knowledge has been described in this chapter as a valuable asset in the Knowledge Economy. Companies acknowledge that their economic success does not rely only on their capability to effectively use their knowledge to innovate, to spark new knowledge, and to take action. They recognise that they are part of a larger system of supplying and supporting firms, customers, policies, and the environment where exchange of ideas, dialogue, co-ordination, and negotiation with those external agents takes place.

The literature review reveals that there is still dispute on the nature of knowledge, in particular its tacit/explicit attribute, as well as what constitutes knowledge and information. The position of this study is to agree with the view of "knowledge-ascontinuum," and hence that knowledge has both tacit and explicit dimensions. I am not concerned with differentiating between information and the explicit representation of knowledge, for the reasons already outlined in the literature review.

My interest in clusters lies in the fact that they hold knowledge. Some authors argue that clusters are the passive recipients of knowledge spillovers, whereas others believe that clusters are actively creating and transferring knowledge. The latter view is the basis of this study; this is based on my preliminary work to assess the viability of studying clusters as research subjects for the thesis, where anecdotal evidence revealed that the clusters I approached do engage in activities supporting this argument.

The area where this study wants to provide a major contribution to the literature is in the transfer of knowledge in clusters. Knowledge transfer implies a movement of knowledge from one point to another to produce an effect or change at the receiver's end. Knowledge transfer entails two key components: knowledge sharing, in which new 
knowledge is circulated; and knowledge reuse, in which existing knowledge is considered in new situations. The review of the literature identified several factors that could hinder the effectiveness of knowledge transfer, including absorptive capacity, the knowledge characteristics, and the context.

Clusters provide a favourable environment for knowledge transfer, as they address - or at least minimise - some of its barriers. For instance, the business values and codes of practice that emerge in a cluster can improve the level of trust amongst members and minimise opportunistic behaviour. Clusters embody a range of related industries, and therefore members share common skills, capabilities, and language that can facilitate the absorption and diffusion of knowledge.

This study also focused on Markus' (2001) "shared work practitioners," the second group of knowledge reusers in a cluster environment. The study assessed the knowledge which is needed by a cluster working towards a project bid, and aimed to help the cluster leverage knowledge transfer among its members, ensuring a quick and innovative response in project bidding.

This study will further explore knowledge transfer mechanisms - how the exchange of knowledge takes place between cluster members. The literature mentions the importance of having the appropriate match between the mechanism and the nature of the knowledge to be transferred, and has pointed out some of the communication channels that can be used. Although knowledge transfer mechanisms have been recognised and used in the literature, there are not many studies providing a systematic approach to identifying and defining them in a particular business process.

Furthermore, given that it is argued that each organisational arrangement will have a specific portfolio of knowledge transfer mechanisms, attention will be focused on one particular business process within the cluster to keep the scope of this study at a manageable size. Therefore, the first question formulated for this study is as follows:

\section{What are the knowledge transfer mechanisms in a cluster when it performs a business activity such as international project bidding?}

As already demonstrated in the literature review, ICT facilitates the communication, storage, location, and sharing of knowledge when individuals are willing to do so; in other words, ICT provides the infrastructure to allow knowledge transfer. 
Despite the fact that the cluster literature acknowledges that ICT plays a key role in enabling collaboration among partners, little has been written about how ICT supports business cluster activities, in particular knowledge transfer. This study aims to be one example in the literature illustrating the role of ICT in supporting clusters and, for this, I formulated the following second research question:

\section{How can ICT be used to improve cluster knowledge transfer during project bidding?}

Answering this question will allow identification of the aspects to consider when exploring how information and communication technologies can support clusters in the management of their knowledge. 


\section{Chapter 3: The theoretical framework}

Chapter 2 has provided the contextual information for this study and how the research questions were formulated. The literature has revealed the challenges encountered by nations and businesses in the Knowledge Economy paradigm, the relevance of clusters for regional economic development in this new environment, how it is critical for clusters to improve their knowledge mechanisms in order to remain a source of innovation, how ICT has evolved from back-office data-processing tools to become a key facilitator in the Knowledge Economy, and the potential use of ICT to support knowledge transfer activities.

It is time to focus on how to address the study's research questions:

- What are the knowledge transfer mechanisms in a cluster when it is performing a business activity such as international project bidding?

- How can ICT be used to improve cluster knowledge transfer during project bidding?

To do this I require two things: a theoretical framework to guide my inquiry, and a methodology to direct my data collection and analysis and to validate how the study was carried out. The latter will be discussed in Chapter 4, while attention to the former is given in this Chapter.

Genre theory is the theoretical framework I adopted to build on further theory. The following section explains the value of having a theoretical framework as the starting point in a qualitative study such as mine. An overview of other theoretical frameworks that were considered for this study follows. Attention is then focused on describing genre theory - the selected framework - and how it has evolved in the Information Systems domain. Finally, I state why genre theory is a suitable perspective for my study and which of its variants I have subscribed to.

\subsection{The value of a theoretical framework}

Creswell (2003) and Miles and Huberman (1999) indicate that theory in the qualitative inquiry could be employed as a theoretical lens, perspective, or framework to conduct 
the study. A theoretical framework presents the main themes/concepts/factors to be studied and explains how they are thought to be interrelated (Creswell, 2003; Miles \& Huberman, 1999). Theoretical frameworks can follow a narrative style, but Creswell (2003) recommends the use of diagrams to represent them. Theory in qualitative studies should not be viewed as a set of pre-determined categories and relationships to be tested - as in the case of quantitative inquiry - but rather as a guide suggesting key aspects on which researchers should focus their attention in order to develop theoretical or conceptual outcomes (Creswell, 2003; Morse, 1998).

Miles and Huberman (1999) believe theoretical frameworks in qualitative research are a good initiative. They indicate that researchers will always have some ideas about what to look for, regardless of their efforts to be unstructured and inductive in their inquiry. The authors cite the example of how a social researcher at a lunchroom would study roles, relationships, routines, and norms, while an architect would study the layout, light, and distribution of elements in the same space.

Another argument put forward by Miles and Huberman (1999) is the researcher's experience. They recommend that the neophyte qualitative researcher should spend some time thinking about the study's design, as this will grant clarity and help them focus on the study's issues. This way they will avoid spending months collecting and analysing data that were once considered important but are no longer relevant. Experienced researchers can use highly inductive and unstructured studies because they know what sort of questions to ask and what events are worth paying attention to, and because they usually have considerable time and resources.

For Creswell (2003), a framework can be drawn from the literature and used as the foundation to build on further theory. In this case the framework is not tested; instead, it is modified or adjusted according to the outcomes of the new study. Usually a refined model emerges which better explains the phenomenon. On a similar note, Miles and Huberman (1999) mention the option for the researcher to develop a tentative conceptual framework at the beginning of the inquiry based on the literature, their experience of the topic, and the study's general objectives. Again, this initial conceptual framework is likely to evolve and develop once it is taken into the field. 


\subsection{Theoretical frameworks considered for the study}

At the time I was setting up the research design, a search in the literature yielded three models that had the potential to be the basis for further theory-building towards explaining how ICT can contribute towards improving a cluster's knowledge transfer in general and knowledge mechanisms in particular. This section introduces the first two frameworks I considered before selecting genre theory.

\subsubsection{Rolland and Chauvel's knowledge transfer framework}

Rolland and Chauvel (2000) argued that many firms in the Knowledge Economy do not partner up simply as an answer to changes in the economic environment, or to reduce transaction costs, but rather to access and combine their knowledge and competencies with those of others, and thus increase their competitive advantage. Firms possess different types and levels of knowledge, which constitute their unique stock. In order to be shared, knowledge needs to be transferred. This requires a structure and some operating principles for its effective implementation.

Rolland and Chauvel (2000) proposed a framework of six critical elements and their respective interaction with one another (see Figure 7): strategic intent, culture, trust, form, transparency, and learning capacity. The first four elements are initial conditions for successful knowledge transfer, while the other two are dependent variables or strategic outcomes.

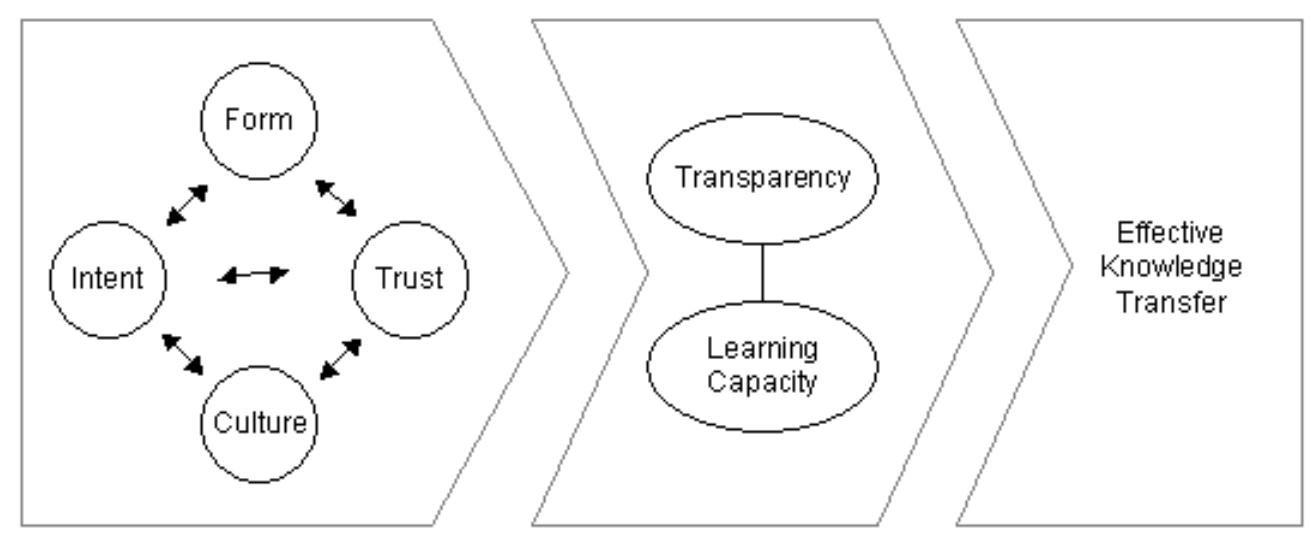

Figure 7. A model of knowledge transfer (source: Rolland \& Chauvel, 2000).

Strategic intent depicts how the firm plans to commit to a collaboration agreement. There are three types of intent in an strategic alliance: access, where the firm uses other partner's competencies for a limited time and sticks strictly to what was previously 
agreed by the parties; internalisation, where the firm aims to capture knowledge and competencies from partners; and integration, where the idea is to complement and combine competencies and knowledge in order to create a common strategic competence, activity or product.

Organisational culture considers the beliefs and values shared by the people in the firm. Culture provides, defines, and stipulates the routines and habits that affect how people learn and communicate, and therefore how they transfer knowledge. According to Rolland and Chauvel (2000), organisations inclined towards external adaptation and changes are better equipped to transfer knowledge and learn from the alliance than those who are closed and inflexible.

Rolland and Chauvel (2000) indicated that the higher the level of trust between partners in the learning-base alliance, the more effective knowledge transfer becomes, for tacit knowledge in particular. Three main factors for trust are: interdependency, where mutual needs ensure parties respect their commitments; the partner's reputation for being a fair player; and prior experience in working with partners, which shows how partners may behave in the future.

The form of the alliance - that is, how it has been designed - will specify the controls and measures followed for knowledge transfer and learning. Rolland and Chauvel (2000) identified three forms of alliances: non-equity form, where there is a contractual relationship between partners and thus the limited knowledge transfer is subject to what the contract states; joint venture form, where partners create a new entity and hence knowledge is transferred from parent companies to the new one; and finally the equity form, where partners engage in serious commitments with each other allowing direct knowledge transfer to occur between them.

Transparency is one of the two strategic outcomes expected from the four factors described above. Transparency was defined as "the extent of communication and knowledge transfer that occurs between partners" (Rolland and Chauvel, 2000:232) Two aspects to consider in transparency are: the nature of the knowledge in question and how it is articulated, and the level of willingness to disseminate and integrate this knowledge.

Transferring explicit knowledge is a relatively transparent process, as this knowledge can be "packed" in a systematic and codified fashion. The tacit knowledge that is 
embedded in organisational routines, market intelligence, and individuals, on the other hand, may not transfer that smoothly. Firms are trying to balance competing and collaborating with partners; in other words, they are willing to share knowledge but at the same time they are trying to create and keep new competencies within their individual boundaries. Transparency will therefore depend on how comfortable or threatened partners feel about transferring knowledge.

Finally, learning capacity is the other expected strategic outcome of the model. It refers to the ability of a firm to internalise new knowledge into their existing stocks and flows. At a practical level, learning capacity will depend on the way a partner codifies its knowledge, how that knowledge is communicated to their partners, and its willingness and ability to change existing or organisational routines to accommodate the new knowledge. Rolland and Chauvel (2000) argued that new knowledge is internalised more easily when partners share similar cognitive bases (e.g. assumptions and frameworks) and communication structures.

Rolland and Chauvel's (2000) model could be the basis of a framework for a cluster due to its emphasis on knowledge transfer between business parties. From studying the model, I infer that ICT could play an important role in supporting the two outcome strategies. Transparency will benefit the most as ICT could be used to ensure the fluidity and exchange of knowledge through soft and hard technologies. Although learning capacity will depend on the cluster's ability to internalise knowledge, ICT could support some of its practical aspects, such as standardising the coding of knowledge, providing the communication structures, and storing knowledge.

Although this framework could assist in identifying the factors or elements that would impact knowledge transfer in clusters, this is not the focus of this study. My interest lies in exploring the knowledge transfer mechanisms, which this framework vaguely refers to in the transparency factor. For this reason, I decided to search for other theoretical frameworks that could fit better in answering the research questions of this study.

\subsubsection{Tallman, Jenkins, Henry and Pinch's knowledge-base framework}

In the context of clusters, Tallman, Jenkins, Henry, and Pinch (2004) looked at knowledge-based theories at firm and cluster levels, and integrated concepts from economic geography (such as spillovers and physical location) and strategic management (such as knowledge stock and flow at firm level) to develop a framework 
which categorises knowledge and how it flows between individual firms and the cluster (see Table 1).

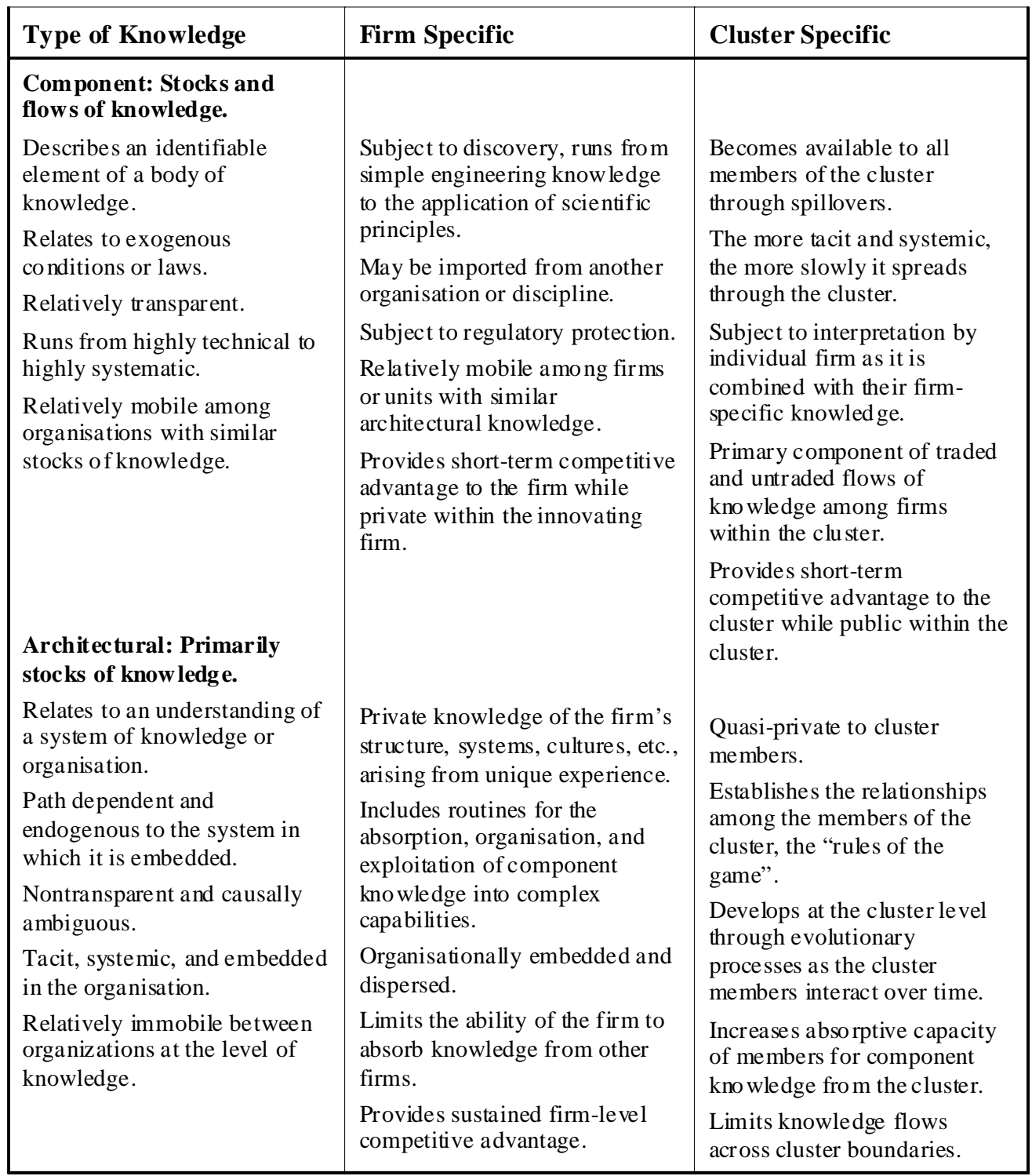

Table 1. Types of knowledge at firm and cluster level (Source: Tallman et al., 2004).

Tallman et al. (2004) describe component knowledge as the specific knowledge and skills for a particular industry or field. The authors provide the examples of design for technology-driven industries, and marketing for consumer-oriented ones. Component knowledge tends to be easy to transfer across a cluster as it is usually logical, easy to package, and understanding it does not rely on the organisation's contextual background. Component knowledge is also highly transferable because firms in a cluster share the same location, use similar ontology of terms, and frequently interact with one another at business and social levels. 
Architectural knowledge is described as organisation-specific, highly complex, and tacit. It is knowledge embedded in a firm's own structures and routines, which has evolved over time and thus is not readily transferable. However, the authors ;make an interesting observation: through their interaction, interdependencies, and common interests cluster members will develop a "cluster-specific" architectural knowledge over time that will differentiate them from others in the industry. Examples of clusterspecific architectural knowledge given by Tallman et al. (2004) are understandings of reciprocity, reputation, and how members relate to each other. In fact, the authors assert that the presence of cluster-specific architectural knowledge increases the exchange of component knowledge among cluster members.

Tallman et al. (2004) also argue that, although knowledge is transferred to achieve competitive advantage at cluster level, some knowledge remains within the firm to reach competitive advantage. The authors describe how competitive advantage from component knowledge will be short-lived for an individual firm once this knowledge is absorbed by the cluster; this knowledge is, again, a short-lived competitive advantage to the cluster over the rest of the industry as it becomes public. Architectural knowledge moves more slowly than component knowledge and therefore gives a more stable competitive advantage to both individual firms and clusters.

Tallman et al's (2004) framework gives insights into the dynamics of knowledge flow that could be expected to be found within a cluster. Their framework has the potential to guide my study, as what is proposed as component and architectural knowledge in clusters could lead to identifying some of the knowledge management mechanisms. The framework, however, focuses on the composition of a cluster's knowledge-base, which is not the main focus of my study. I am trying to develop further theory on knowledge transfer mechanisms in the context of clusters. Nevertheless I have referred back to their framework, particularly when discussing my findings in Chapter 12.

\subsection{The genre theory framework}

Genre theory is the theoretical framework adopted. Compared with the other two, this framework better meets the purposes of the study. This section and the subsequent ones describe genre theory, how it has been applied in the information systems domain, and finally how it was applied in this study. 
The current business environment and technological advancements in information and communications are producing novel ways of collaboration across time, distance, and organisations, such as virtual teams and networks (DeFillipi, 2002; Snow et al., 1992; Yates \& Orlikowski, 2002). This sparked Yates and Orlikowski's interest in the communication interactions of these communities and proposed the use of genres to understand such interactions.

Traditionally, genre has been used to describe and classify rhetorical discourse and literary work, such as the novel, the sonnet, and the essay. From the mid-1980s onwards there has been a growing interest in the fields of applied linguistics, communications, systems design, and education in employing genre to define and organise the underlying social situations and activities that are reflected in the text - or any other communicative action (Bawarshi, 2000; Freedman \& Medway, 1994; Yates \& Orlikowski, 2002).

Yates and Orlikowski (1992) adopted the concept of genre as the analytical unit for their structurational approach to:

examine the production, reproduction and modification of different types of organisational communication over time and under different circumstances (p. 301).

For them, various genres are the physical manifestations of social structures that continually interact with individuals through social rules.

As an example of a social structure, Yates and Orlikowski (1992) mentioned the business letter genre. The social rules for this genre stipulate that individuals should use business letters when there is need for a documented communication outside the organisation's boundaries. The social rules - or "genre rules," as these authors call them - also specify that the business letter should be written in formal language and it should include an inside address, salutation, body, and complimentary close.

By drawing from these genre rules when writing a business letter, individuals are legitimising these rules and consequently ratifying the social structure (i.e. the business letter genre). "A genre exists only in the recognitions and attributions of the users" (Bazerman, 1994:81).

According to Yates and Orlikowski (1992), genre rules can be made explicit by codifying them into specific standards, which are then circulated within the organisation to regulate when and how a genre should be used. They can be embedded in a medium, 
such as a paper-base form or an electronic template, thus guaranteeing adherence to the rules. Alternatively, genre rules can be tacitly invoked through common practice: it is what is done in a particular situation.

Orlikowski and Yates (1994) define genre from an organisational perspective as:

... socially recognized types of communicative actions - such as memos, meetings, expense forms, and training seminars - that are habitually enacted by members of a community to realize particular social purposes (p.542).

A genre works as an "institutionalised template" for social interaction. Genres are characterised by their purpose and form (Orlikowski \& Yates, 1994; Yates, Orlikowski $\&$ Okamura, 1999). The purpose expresses the social motives, themes, and topics of a genre, such as proposing a project, meeting to review project progress, and/or making a presentation to show results or findings. The genre's purpose has previously been defined and recognised by the organisational community.

Form, on the other hand, relates to the way the communication is presented in terms of medium (document, telephone call, face-to-face interaction), structure (if there is a predefined format to follow for the particular type of communication in terms of headings, addressing the reader, main body, and sign-off), and linguistics (level of formality, vocabulary, and graphic expressions).

For Bawarshi (2000) genre is both functional and epistemological: functional because the more a genre is used - out of tradition or mandate - by the community to respond to a repeated social situation, the greater the legitimacy as the proper communicative action to take; and epistemological because the validated genre shapes how the community conceptualises and experiences the situation, thus generating an expectation of appropriate and possible communicative actions.

Nevertheless, genres are not rigid social structures. Individuals can defy the genre rules that dictate actions to be followed in a recurrent situation within the organisation, and consequently modify the genre. For instance, Yates and Orlikowski (2002) mention the case of changing a meeting from its traditional face-to-face form into audioconferencing to include off-site participants.

Repeated and significant modifications of the genre rules will eventually generate a new genre. Yates and Orlikowski (1992) illustrate this with the genesis of the memo genre. 
The socioeconomic, technological, and management ideology changes of the 1870s and onwards transformed the small, single-level, and owner-managed firm that commonly used oral exchanges for internal coordination and control into the big, departmentalised, multi-level management organisation that required written internal communication. The social rules of the business letter genre were initially drawn upon for these written internal communications. In time the headings, language used, range of topics per communication, format, and advent of the typewriter made these internal communications so different from the business letter that the memo genre was born.

\subsection{Genre repertoire and genre systems}

Genres can be examined as stand-alone units; however, in the organisational context they are usually related to other genres. There are instances when studying several related genres will bring a more holistic view of the community, its social actions, and its communication practices. Orlikowski and Yates (1994) suggest two notions of genre relations: genre repertoire and genre system.

In a genre repertoire, individuals in a community will invoke a distinctive communicative set of genres to perform their activities. Østerlund (2006) mentions laboratory test results, radiology results, and discharge notes as part of the genre repertoire available to doctors and nurses in an Emergency Room.

Orlikowski and Yates (1994) employed the notion of genre repertoire to identify the genres that a temporary virtual team of computer designers enacted via e-mail whilst working towards a standardised computer language. The genre repertoire in this instance consisted of memo, proposal, dialogue, and ballot. The authors argue that the presence (or absence) of genres, such as business letter and progress report, in the repertoire indicated the nature of the community - in this case informal, temporal, amongst colleagues, highly participative, and where individuals felt answerable for their own actions. Finally, genre repertoire allowed Orlikowski and Yates (1994) to observe deliberate or inadvertent variations in the composition of genres over time as participants responded to changes in their context, activities, and level of knowledge.

On the contrary, genre systems entail that each communicative action follows a predefined sequence to achieve the desired interaction (Bazerman, 1994). The example by Yates and Orlikowski (2002) of "handling a major project contract" - a situation 
relevant to the clusters of my study - would be initiated by a client's request for proposals, followed by the responding candidates' proposals, then the evaluation report of the proposals, and finally the contract with the successful candidate.

For Yates and Orlikowski (2002), genre systems aid the way these interactions are expected to take place in terms of:

- Purpose: What the community expects to gain. For instance, "hiring personnel" aims to find the most suitable person for the job.

- Content: What genres are included and what information each of these should include. Examples for the case of "hiring personnel" include a job advertisement, applicant's covering letter and CV, invitation to interview, the interview itself, psychometric tests, and the job offer.

- Actors: Who is involved and their roles.

- Form: Media, structure, and linguistic.

- Time: Specific deadlines applicable to each genre in the system.

- Place: Physical or electronic.

To demonstrate how genre systems can explain the employment of a new electronic media for collaborative interactions in the organisational context, Yates and Orlikowski (2002) looked at three teams in a high-tech organisation using Team Room, a collaborative application in Lotus Notes. The authors analysed the messages posted in Team Room by the three teams, using a genre-coding scheme based on purpose and form. In order to interpret the genre systems, they also conducted interviews with team members to gain additional understanding about the organisational conditions under which the collaboration took place. As a result, Yates and Orlikowski (2002) identified three genre systems employed by the teams: the meeting, the collaborative authoring, and the collaborative repository.

The meeting genre system included all the communicative activities associated with face-to-face meetings, such as meeting logistics, meeting agenda, the meeting itself, and meeting minutes. Yates and Orlikowski (2002) noted that this genre system was a direct transfer of the paper-based world to electronic media. 
The collaborative authoring genre system, on the other hand, incorporated all the communicative activities employed to create documents in a cooperative effort by the team, such as a circulated draft, request for comments, responses to the draft, and the final version. The authors pointed out that this system is similar to the paper-based or email-based ones; however, Team Room enabled many-to-many participation and interaction, thus allowing the team to more openly exchange ideas and feedback.

Finally, the collaborative repository genre system entailed establishing a discussion forum, where a topic is proposed, and inviting the rest of the team to contribute comments and opinions. The teams employed this genre system for various activities such as coordinating schedules, brainstorming, initiating discussions, and consolidating topic information.

Table 2 details how the genre systems framework can describe the three teams' communicative interactions in terms of purpose, content, actors, form, time, and place.

\begin{tabular}{|c|c|c|c|}
\hline $\begin{array}{c}\text { Genre System } \\
\text { Dimension }\end{array}$ & Meeting & Collaborative authoring & Collaborative repository \\
\hline $\begin{array}{l}\text { Purpose: } \\
\text { What the community } \\
\text { expects to gain. }\end{array}$ & $\begin{array}{l}\text { Coordination of a } \\
\text { meeting among a } \\
\text { specified set of people } \\
\text { to achieve an explicit } \\
\text { purpose (e.g. organise a } \\
\text { Total Quality } \\
\text { Management project). }\end{array}$ & $\begin{array}{l}\text { Request suggestions and } \\
\text { incorporate them into a } \\
\text { document considered } \\
\text { important to the team and } \\
\text { needing buy-in from other } \\
\text { team members. }\end{array}$ & $\begin{array}{l}\text { Designating a specific } \\
\text { electronic space to collect } \\
\text { and store input about a } \\
\text { specific issue. }\end{array}$ \\
\hline \begin{tabular}{l}
\multicolumn{1}{c}{ Content: } \\
What genres are \\
included, and what \\
information each of \\
these should enclose.
\end{tabular} & $\begin{array}{l}\text { Meeting logistics } \\
\text { messages, agenda, the } \\
\text { meeting itself, and } \\
\text { minutes }\end{array}$ & $\begin{array}{l}\text { Circulated draft, } \\
\text { responses/feedback, } \\
\text { dialogue, and final } \\
\text { version. }\end{array}$ & $\begin{array}{l}\text { Topic message and } \\
\text { contributions (with } \\
\text { comments and opinions) } \\
\text { by other team members. }\end{array}$ \\
\hline $\begin{array}{l}\text { Actors: } \\
\text { Who are involved and } \\
\text { what their roles are. }\end{array}$ & \multicolumn{3}{|c|}{$\begin{array}{l}\text { It depended on each team: if the team suffered from tension and rivalries, then the } \\
\text { owner of the project would initiate the genre system; if the team was cooperative, } \\
\text { then anyone would initiate the genre system. }\end{array}$} \\
\hline $\begin{array}{l}\quad \text { Form: } \\
\text { Media, structure and } \\
\text { linguistic. }\end{array}$ & Mainly face-to-face & $\begin{array}{l}\text { Posting drafts where all } \\
\text { members could see them } \\
\text { and make comments } \\
\text { instead of circulating draft } \\
\text { to selected people. }\end{array}$ & $\begin{array}{l}\text { Replace face-to-face } \\
\text { meetings by allowing } \\
\text { asynchronous } \\
\text { contributions in the } \\
\text { discussion forum. }\end{array}$ \\
\hline $\begin{array}{l}\text { Time: } \\
\text { Specific deadlines for } \\
\text { each genre in the } \\
\text { system to take place. }\end{array}$ & $\begin{array}{l}\text { The meeting organiser } \\
\text { announces date and } \\
\text { time. }\end{array}$ & \multicolumn{2}{|c|}{$\begin{array}{l}\text { Not clearly stated. However prompt responses are } \\
\text { expected once the material is posted in Team Room. }\end{array}$} \\
\hline $\begin{array}{c}\text { Place: } \\
\text { Physical or electronic. }\end{array}$ & $\begin{array}{l}\text { Physical location for } \\
\text { meeting and Team } \\
\text { Room }\end{array}$ & Team Room & Team Room \\
\hline
\end{tabular}

Table 2. Three genre systems (source: Yates \& Orlikowski, 2002). 
Genre, genre repertoire, and genre systems become useful analytical tools in identifying the various communications exchanged within a specific community. In so doing, researchers and practitioners gain knowledge about the community's collaborative practices and how such tasks are structured, coordinated, and executed over time and space (Firth \& Lawrence, 2003; Yates \& Orlikowski, 2002). The remaining question is whether to examine a genre, a genre repertoire, or a genre system. For Orlikowski and Yates (1994) this would depend on "the exact relationship of the genres and the purpose of the research" (p. 545).

\subsection{The genre framework in Information Systems (IS)}

In the IS domain, genre has been used to analyse IT-mediated communications (Firth \& Lawrence, 2003; Pollach, 2006). A review in the IS literature confirms Ihlström's and Henfridsson's (2005) argument that genre theory research has two main contexts: inside the organisation and outside the organisation.

The early studies of genres in IS took place mostly within organisational boundaries, with regular contributions by Yates and Orlikowski $(1992,1994,2002)$ and by these two authors in collaboration with a third researcher (Yates, Orlikowski \& Okamura, 1999; Im, Yates \& Orlikowski, 2005). Studies in this context look at the communicative interactions facilitated by the electronic medium to determine whether a community in the organisation has taken advantage of the opportunities the new medium offers.

Along these lines, Bergquist and Ljungberg (1999) examined the incoming e-mail messages of an individual in a large Swedish IT-company over the course of a week using genre analysis ${ }^{6}$. The purpose of their study was to map the genres employed by the individual when communicating through this electronic medium, and examine how he used them. Their preliminary findings confirmed that genres are "dynamic and living phenomena" (p.11), as the individual negotiated with his colleagues and manager to determine which genres to enact in particular situations. The authors also observed that some genres could have been better communicated through other IT-mediated medium instead of the e-mail system. For example, they suggested the company's intranet for the genre "organisational announcement."

\footnotetext{
${ }^{6}$ Bergquist and Ljungberg (1999) published the preliminary findings of a large and complex study examining the company's communication interactions at three points in the company's life-span: 1930s (paper-based communications), early 1980s (e-mail-based communications), and late 1990s (intranetbased communications).
} 
The second context of genre theory research is outside the organisational boundaries, where different forms of Internet and digital technologies have created a broader, open, and sometimes public environment. There is currently a high level of experimentation by computing and Internet users, to the point that these relatively recent communication media have been identified as powerful enablers for the variation of existing genres or the creation of new ones (Crowston \& Williams, 2000; Ihlström \& Henfridsson, 2005; Shepherd \& Watters, 1998). In this context, researchers have applied genre analysis to online newspapers (Ihlström \& Henfridsson, 2005), spam (Cuckier, Cody \& Nesselroth, 2006), and online product reviews (Pollach, 2006).

Crowston and Williams (2000) used the genre framework in 1000 web pages to identify which were recreating traditional genres and which were emergent genres. The authors also argue that the pages are part of a genre system, as many pages are highly dependent on others due to interactive web design practices. However, this argument views genre systems as biological, instead of the procedural or sequential view depicted by Yates and Orlikowski (2002) and Bazerman (1994).

The fact that the combination of computing, the World Wide Web, and the Internet is such a powerful vehicle for genres has prompted authors like Rhem (2002), Ihlström and Henfridsson (2005) and Pollach (2006) to call them digital genres. Shepherd and Watters (1998) coined the term cybergenre to define the genres found in this new digital medium and proposed the taxonomy depicted in Figure 8 to classify them.

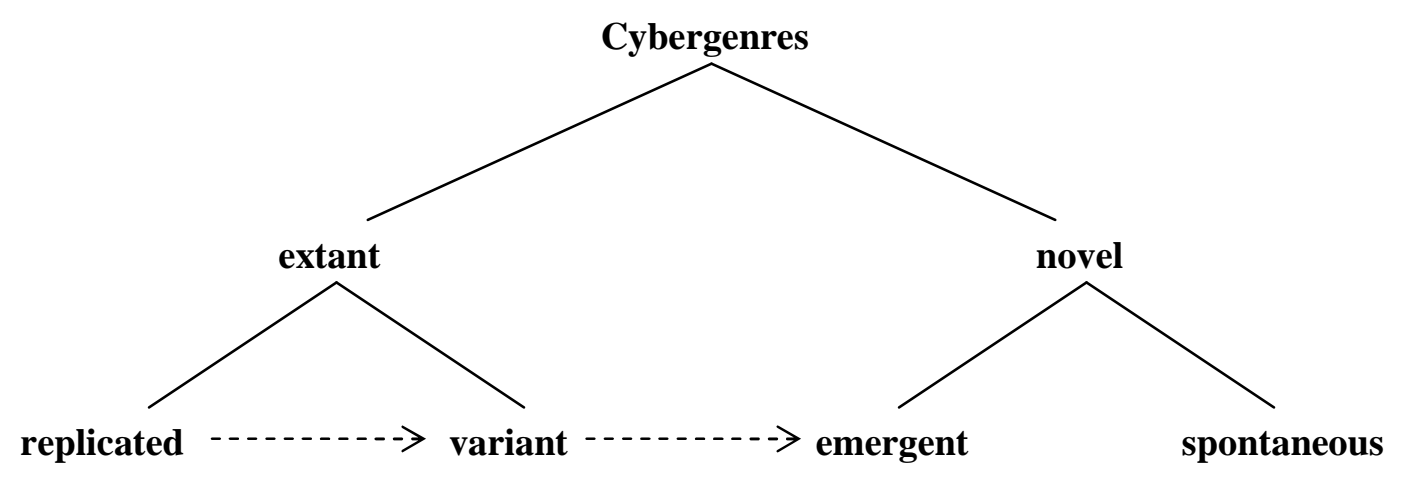

Figure 8. The evolution of cybergenres (source: Shepherd \& Watters, 1998). 
Shepherd and Watters (1998) classified cybergenres into two classes: extant and novel. The extant class comprises genres already existing in other media (e.g. paper, audio, and video) that have made their way into the Internet. Usually these cybergenres started as exact copies of genres in the original medium (replicated). As users of these genres become familiar with the capabilities and functionality of the new medium, they start to be more adventurous with their reinterpretation of the original genres, leading to genre variations over time (variant).

The novel class of cybergenre was defined by Shepherd and Watters (1998:3) as any genre "wholly dependent on the new medium." Due to their high level of functionality, these genres would work only in the digital medium. In addition, novel cybergenres subscribe to one of the following conditions: 1) they have followed a progression path from replicated to variant and beyond (emergent); or 2) they are truly digital genres, with no equivalent in other media (spontaneous) ${ }^{7}$. This progression from replicated to emergent cybergenre is represented in Figure 8 by dotted arrows.

Shepherd and Watters (1998), and Askehave and Nielsen (2005) proposed that in addition to purpose and form, cybergenres should be characterised in terms of functionality of the electronic medium, consequently adding a new dimension to genre analysis. Examples of such functionality mentioned by Askehave and Nielsen (2005) are multimedianess (where text, images, sound, and animation are combined to produce a screen page) and hypertext (where related texts are connected through links, thus allowing non-linear transmission of content).

Ihlström and Henfridsson (2005) subscribed to the concept of cybergenres to examine the evolutionary path of three Scandinavian online newspapers from replicated genres into novel ones. For them, the functionality of the new medium - which enables features such as archives, search engines, multimedia, polls, and interactivity - has made the online newspaper a genre distinct from its paper-based counterpart.

However Ihlström and Henfridsson (2005) state that the technical capability of the new digital medium is insufficient to explain such progression, and hence they adhere to the notion of structuration: "genres do not emerge in a social vacuum" (p.175). In their study, changes in the media organisations' strategies due to social (e.g. attitude toward

\footnotetext{
${ }^{7}$ Shepherd and Watters (1998) mentioned Home Page and Hot List as examples of novel spontaneous cybergenres.
} 
online publishing), economic (e.g. dot-com downturn), and technological (e.g. functionality) factors have shaped the three online newspapers over time.

One interesting finding by Ihlström and Henfridsson (2005) was the interdependency between the paper-based newspaper and the online genres. Cross-references between these two genres are now common practice, thus reinforcing brand recognition and complementing each other's content. The authors conclude that successful newspaper houses are those who learn to manage their interdependent genre repertoire, taking into consideration the changes in the environment.

To conclude this section, attention will be paid to Firth's and Lawrence's (2003) observations, which confirm the relevance of genre as a theoretical framework to advance knowledge in the IS discipline. Firstly, their review of the literature about the use of genre analysis in IS notes that it has been applied to a wide range of synchronous (e.g. computer-mediated communications), asynchronous (e.g. e-mail), and collaborative (e.g. Team Room) IT systems. Secondly, Firth and Lawrence (2003) point out that many authors had explored the communication structures with genre theory, not only in terms of how genres are shaped by the IT systems but also in how the IT systems - such as interface and design - are modified to fit better with genres familiar to users. Finally, they argued that genre analysis applied to a community's computingmediated communications increases our understanding of the IT artefact and associated social and management implications.

\subsection{Using genre theory as the theoretical framework for this study}

While reading the knowledge transfer literature, I noticed that many authors referred to various genres when discussing knowledge transfer mechanisms. Appleyard (1996) lists in her matrix (see Figure 5 on page 38) several knowledge sharing mechanisms such as the newsletter, benchmarking studies, and meetings. Newel (2004) talks about project reviews as a mechanism to capture the lessons learned in a project for future reuse. Owen et al. (2004), on the other hand, mention in their study that intra-project learning was recorded through project reports, status reports, and memos.

This study proposes that genres can be considered knowledge transfer mechanisms because genres communicate knowledge to members of the community following specific social rules. For that reason, genres and the way they are invoked contribute 
towards explaining how knowledge is shared and reused by a community. To answer the first research question of this study I needed to identify the knowledge transfer mechanisms used by the cluster community, and the concept of genre was the analytical unit that helped me do so.

A brief look at the clusters' communicative interactions revealed the use of a wide range of genres in their repertoire to carry out activities, such as marketing, promotion, project bidding, monitoring opportunities, relationship building, organising events, applying for funding, and networking. Consultation with cluster members identified project bidding as one activity where it was felt knowledge transfer could be improved with the introduction of ICT.

In general terms, project bidding in a cluster involves identifying the opportunity, alerting members to it, setting up a team to pursue the opportunity, and submitting a proposal. If the team is successful it negotiates the final contract with the client and begins the project.

Since project bidding encompasses a series of sequential steps and interactions between the various cluster stakeholders, I subscribed to the notion of genre systems to map the sub-systems and genres that play a part in this activity. Mapping the genres employed during project bidding allowed me to assess the parts of the system where ICT could be used to improve knowledge transfer - helping to answer my second research question.

Although cluster members tend to be located close together, thus facilitating face-toface interactions for project bidding, there are instances where ICT can support their communication, co-ordination, location/storage, and collaboration activities. It is therefore important for clusters to have the proper information systems in place to enable knowledge and information to flow as smoothly as possible between partners.

My study expected to discover an evolution of some genres invoked by the cluster during project bidding as a result of introducing an ICT-based medium. To analyse the possible changes in these genres I needed a genre-coding scheme; that is, the set of elements to which I should pay attention when examining each genre. I followed Yates and Orlikowski's (2002) coding scheme for genre systems (purpose, content, actors, form, time, and place), which is an expansion of the traditional purpose-form genre analysis. 
Acknowledging that clusters are embedded in a socio-economic, political, and historical context had led me to consider the traditional stream of genre theory. Any proposed ICT solution to support knowledge transfer was going to be moulded by the clusters' contextual factors. Furthermore, the traditional stream aligned better with my interest in understanding the organisational and management aspects when introducing new technology to a system.

The literature does not discuss in detail any potential disadvantages or limitations of using genre theory. However I can infer that, due to the focus of examining in detail the communication practices by a community, one could overlook the "bigger picture" when immersed in genre analysis despite the importance given to the contextual factors. In addition, applying genre theory is not infallible and one could overlook important but less obvious genres that could affect the final outcome of the analysis.

Finally, genre theory is relatively new to IS research and it has not been applied in the context of business clusters until now. This study therefore could reveal additional elements to consider when using this framework in both of these contexts. Creswell (2003) observes that a theoretical framework may suffer modifications and/or adjustments once it has been tested out in the practical world. Yates and Orlikowski (1992) acknowledge that the genre approach needs to be further developed via empirical studies such as this one. 


\section{Chapter 4: Philosophical Position and Methodology}

This chapter details the philosophical position and the methodology followed in this research inquiry. It includes my statement to the IS community of how I conducted the research and how the community should assess whether the problems, solutions, and validation criteria declared followed a conventional path to advance the state of IS scholarly knowledge (Hirschheim, 1992; Hirschheim \& Klein, 1994).

\subsection{The philosophical paradigm of this study}

The IS community is a multi-disciplined one, with members coming from various backgrounds such as computer science, business studies, management, economics, and behavioural science. These members have brought and applied the perceptions, concepts, and methods of their background disciplines to IS problems, and by so doing have enriched the IS domain (Benville \& Landry, 1992; Lee \& Liebenau, 1997).

At the same time, this has challenged the IS community's philosophical research paradigm to incorporate valuable contributions from other disciplines without threatening the integrity of IS purpose and its importance (Benville \& Landry, 1992; Klein, Nissen \& Hirschheim, 1991; García \& Quek, 1997). For instance, Orlikowski and Baroudi (1991) argue that the IS literature of their time was dominated by the positivist paradigm, and thus IS was restricting itself unnecessarily. They proposed that IS should be receptive to new paradigms from other social sciences that could bring insightful perspectives about the relationships between ICT, people, and organisations.

Ultimately, IS has accepted that there is more than one valid philosophical research paradigm, and numerous authors have proposed various frameworks to introduce them. This section describes some of the philosophical paradigms accepted in IS research. The philosophical stance that this study followed is then stated and justified.

\subsubsection{The philosophical paradigms in IS inquiry}

Lee and Liebenau (1997), Markus (1997), and Creswell (1994) indicate that there are two main paradigms in any social inquiry in general and in IS in particular: quantitative and qualitative. An inquiry following a quantitative path will test the variables of a proposed theory, measuring them by using numbers and statistical analysis to determine 
if the proposed theory can be generalised to the rest of the population. This paradigm is also referred to as the traditional, positivist, experimental, or empiricist paradigm. The qualitative paradigm, on the other hand, aims to understand a social or human problem in its natural context by constructing a holistic picture based on the views of the participants. Other terms used when referring to this paradigm are the constructivist, naturalistic, postpositivist, or postmodern approach.

Both paradigms encompass different assumptions of how an enquiry should be carried out in relation to the view of reality (ontology), the relationship between the researcher and the subjects of the study (epistemology), the values in the study (axiology), the type of language used (rhetoric), and the research process and methodology (Creswell, 1994; Lincoln \& Guba, 1985).

In a quantitative study, there is only one reality that can be measured because it is inherent to the objects of the study, regardless of the observer's perceptions. The quantitative researcher keeps their distance from what is being studied and attempts to control personal bias in order to remain objective in the assessment of the phenomenon. Likewise, the researcher's political, ideological, or moral values are not considered in the study, which is reflected in the use of impersonal language and by reporting "facts" in the written document. Finally, the methodology employed in this paradigm will be deductive in nature, where pre-determined theories, hypotheses, concepts, and variables are tested using statistical and mathematical instruments to understand cause-and-effect relationships (Creswell, 1994; Hirschheim, 1992; Lincoln \& Guba, 1985).

The positivist approach has been successful in many research areas of IS such as data quality (Klein, Goodhue \& Davis, 1997), the usability and quality of information systems (Ghasemzadeh \& Archer, 2000), and the impact of technology on some organisational activities (Ferrán-Urdaneta \& Storck, 1997). Nevertheless, it has been unsuitable for explaining the societal, organisational, group, and human aspects of IS (Lee and Liebenau, 1997).

Qualitative inquiry assumes that reality is perceived and structured by the researcher, the participants, and even the audience interpreting the study, and therefore these multiple realities are acknowledged and reported. The qualitative researcher does not distance themselves from those being studied; instead, they get involved by either experiencing or observing participants' interactions over a period of time. The researcher recognises that their values and those of the people being studied influence 
the study, and thus the language employed tends to be personal. The methodology that best fits this line of enquiry is inductive in nature, incorporating factors based on the participants' and the researcher's experience of the phenomenon in its context. Such factors are then analysed to assess the existence of patterns or theories that can explain the situation (García \& Quek, 1997; Lincoln \& Guba, 1985).

Another known framework in IS literature is that mentioned by Jönsson (1991), Ngwenyama and Lee (1997), and Orlikowski and Baroudi (1991), which articulates three research philosophies: positivist (i.e. quantitative), interpretive (i.e. qualitative), and critical. Both the positivist and interpretive views share the same features as the quantitative and qualitative paradigms from the previous framework, and thus their descriptions will not be repeated here.

The critical view, however, has an evaluative dimension; that is, it goes beyond predicting (positivist) or explaining (interpretive) a phenomenon. Critical studies assess the status quo of a social system - such as an organisation or an information system through dialectical analysis to reveal the historical, ideological, and contradictory nature of "taken-for-granted" social practices about position, tradition, resources, and power. The aim of critical studies is to make individuals aware of the limiting conditions of their surroundings and induce them to challenge these structures in order to reach their potential (Jönsson, 1991; Orlikowski \& Baroudi, 1991). This paradigm is also termed neohumanism (Hirschheim \& Klein, 1994).

Critical inquiry assumes that reality cannot be isolated from its contextual and historical dimensions. Reality is created and recreated; nevertheless it is not something permanent, as reality can be modified after recognising the restrictions in the prevailing economic, political, and cultural contexts (Jönsson, 1991; Orlikowski \& Baroudi, 1991). The critical researcher bonds with those being studied and they are not mere observers of the phenomenon; they influence and are influenced by the study. The critical researcher believes its duty to go beyond understanding how participants perceive, view, and respond to a situation, by critiquing and pointing out to participants those unfounded beliefs, assumptions, and constraints that are restricting them so they can free themselves. The methodologies suitable for critical inquiry are long-term historical studies and ethnography (Ngwenyana \& Lee, 1997; Orlikowski \& Baroudi, 1991).

In 2003 Creswell revisited his position about the philosophical assumptions in research. He argues that progress on how research has been undertaken, and the acceptance of 
other philosophical assumptions in the literature over the past decade, give merit to the re-evaluation of the stance that he once propounded back in 1994. Creswell's Research Design (2003) discusses four streams: postpositivism, social constructivism, advocacy/participatory, and pragmatism.

Postpositivism replaces Creswell's (1994) old term of quantitative (or positivist) assumption. This label attempts to indicate that the positivist stance has moved forward and accepts the fact that claims of absolute truth are not accurate, and thus postpositivist researchers cannot prove hypotheses, but rather indicate failure to reject them. Other positivist beliefs still remain in postpositivism, including a deterministic view of causeand-effect, an attempt to reduce ideas into small sets of statements that can be tested using measurement-like instruments, a belief that reality exists "out there," and a researcher who remains objective.

Creswell's (2003) social constructivism appears to combine the concepts of qualitative and interpretive approaches discussed in the two previous frameworks. A point raised is the fact that subjective meanings of reality have been socially and historically reconciled by people, and therefore social constructivism looks at the context and the "process" of interaction among individuals. In a similar manner to the previous qualitative or interpretive positions, the researcher acknowledges that their own views, values, and background will influence the way they see the study.

The advocacy/participatory approach maintains a resemblance to the critical inquiry discussed by Jönsson (1991), Orlikowski and Baroudi (1991), and Ngwenyama and Lee (1997). However, Creswell (2003) explains more about the role of the researcher and the participants in an advocacy/participatory approach inquiry. He indicates that the researcher will work in collaboration with participants to decrease the amount participants are marginalised from decisions or actions that affect them. Consequently, participants have opportunity to assist in the design of questions, data collection, and the analysis of this.

Finally, Creswell (2003) proposes the pragmatic position, which does not adhere to any particular philosophical stance or reality; the research problem will dictate that. Therefore researchers are free to call on various philosophical assumptions, worldviews, methods, techniques, and procedures of research, and to generate knowledge regarding the problem in question. This stance applies mixed methods, drawing on both quantitative-like and qualitative-like methodologies. 


\subsubsection{Advocacy/participatory as the study's philosophical paradigm}

This research followed the advocacy/participatory philosophical paradigm as stated by Creswell (2003). The decision was based mainly on the nature of the problem that has been put forward, how the research question has been stated, and the way I want to address it given my values and beliefs.

Firstly, advocacy/participatory studies explore a problem that has not been welldocumented in the scientific community, especially when the variables influencing the phenomenon are yet to be identified. In the case of this study, a careful review of the literature revealed a need for research that develops models for knowledge transfer mechanisms in the context of collaborative arrangements, such as clusters, in order to increase the understanding of how these mechanisms are used in such settings. The cluster literature acknowledges that ICT plays a key role in enabling collaboration amongst partners; however, there are very few cases that explore in detail how ICT has been used to support cluster initiatives, and none illustrating how ICT could enable knowledge transfer in clusters.

A second reason to follow the advocacy/participatory stance is the fact that the study cannot be isolated from its contextual and historical dimensions. The three main concepts of this research - clusters, knowledge, and ICT - are strongly intertwined with their context. Clusters' development and performance are influenced by political, socioeconomic, historical, and personal factors which I believe affect how knowledge transfer takes place within the cluster. The literature indicates that knowledge is being created, disseminated, and enhanced in social settings, which I need to consider in order to understand how ICT could support knowledge transfer.

A third motivation for selecting this approach is that it allows me to interact with people. By stating that I am following the advocacy/participatory paradigm, my role as a researcher would not be viewed as compromised by the fact that I have become closer to cluster members and other stakeholders. As a researcher, I want to get involved by observing, interacting, and collaborating with participants over a period of time.

A fourth reason why I found the advocacy/participatory stance appealing is because it enables me to actively contribute towards achieving good knowledge transfer mechanisms, rather than merely interpreting what the cluster experienced to address their challenges. This allowed me to provide useful feedback and tools to the clusters. 
In addition, the advocacy/participatory paradigm fitted in with my belief that participants should play an active role in designing their own solutions. It is important for me to acknowledge the contributions of cluster members and other stakeholders to the research, and to consider myself a part of a team that is addressing a problem.

Finally, I focussed on the participatory aspect of this paradigm rather than the advocacy one. This is because the research participants do not belong to any marginalised social group that needs a representative. On the contrary, they are pro-active towards change that may help improve their situation. This study has the potential to make participants aware of limitations of the current knowledge transfer mechanisms, and induce them to challenge these structures and reach better knowledge transfer practices.

\subsection{Selecting the study's research methodology}

Any study needs a strategy or methodology to direct its inquiry with regard to data collection, analysis, reporting, and validation procedures (Creswell, 2003; Denzin \& Lincoln, 1998; Morse, 1998). For this reason, I looked into three methodologies: case research, grounded theory and action research.

Yin (1994) and Leedy (1997) define case research as a qualitative enquiry where the researcher studies a contemporary phenomenon - i.e. 'the case' - within its natural context at a particular moment in time. The researcher uses multiple sources to collect the evidence needed for their study, including the points of view of the participants. Benbasat, Goldstein, and Mead (1987) also mention that case research does not involve any experimental control or manipulation of the participants. Case research methodology has been used by researchers to study particular phenomena with unique or exceptional qualities, but also it has been useful to make comparisons, build theory, and propose generalisations (Leedy, 1997).

Grounded theory was developed by Glaser and Strauss (1967). This methodology is used to derive theory from data which has been systematically gathered and analysed (Strauss \& Corbin, 1994). To achieve this, grounded theory interplays stages of data collection with analysis to refine the interrelationship of emerging categories (Creswell, 2003). The researcher is expected to have an open mind to what they will find in the data, rather than coming into the process with a theoretical framework or hypothesis to 
test or verify (Mansourian, 2006). Consequently, this is a very inductive and interpretative research method.

Case research and grounded theory tend to report on what was observed, rather than on what was carried out. In addition, these methodologies limit the level of involvement by the researcher in causing change. Furthermore, grounded theory advocates not using a pre-conceived theoretical framework for the inquiry, which is opposite to what I have stated in Chapter 3. For these reasons, neither of these methodologies was adopted.

Action research was the third methodology considered for this study. In action research, researchers and participants collaborate in the analysis of a problem within a social context to produce two outcomes: a solution to the practical problem, and the enhancement of knowledge. To achieve this, researchers and practitioners will make some assumptions based on their observation of the problem and will formulate actions to change the status quo (Baskerville, 1999). A description of action research and the rationale for using it as the research method for this study, as well as how it will be applied, are discussed in Section 4.3 and Section 4.4 respectively.

\subsection{The action research methodology}

The term action research has, unfortunately, been used to identify many different types of activities, and therefore it has become confusing to understand this research strategy. Even worse, some studies claim to be based on action research but in fact share only a couple of its characteristics. This is the case of positivist studies in a "field" context, or studies that describe organisational consultation based on mere information gathering and feedback (Reason \& Bradbury, 2001).

French and Bell (1999) and Lau (1997) explain how action research could be described as a process or an approach to problem solving. The former alludes to a sequence of actions involving the collection of data about an ongoing system, suggesting certain actions based on hunches or hypothesis performed after analysing the collected data, altering selected variables in the system according to the hypothesis, and evaluating the effects of such actions by collecting more data. Prototyping, multiview, and participant observation are two techniques employed in the IS discipline that fits this description ${ }^{8}$.

\footnotetext{
${ }^{8}$ It is important to point out that there is debate on whether to consider these techniques as more than just processes. For further details, see Baskeville and Wood-Harper (1998) and McKay and Marshall (2001).
} 
On the other hand, action research described as an approach or methodology - which is the stance for this study - is more holistic. This implies that action research could be used as a guide to assist in making sense of the research experience (French \& Bell, 1999; Lau, 1997). According to French and Bell (1999), when action research is viewed as an approach, there are three important features to consider: how action research fits into the scientific method of inquiry; the type of relationships between researchers, practitioners, and laypersons; and its contribution to knowledge.

This Section explains action research as an approach and how it is used to carry out research, while Chapter 5 will specify the action research design I followed for this study. The next Sub-sections will provide a description of action research and how it fits into the advocacy/participatory paradigm (Creswell, 2003). Attention will then be focused upon defining action research in the context of information systems.

\subsubsection{Action research in the literature}

A search of the literature discovered many variations of action research depending on its emphasis and the field of inquiry. For instance, Reason and Bradbury (2001) incorporate in their Handbook of Action Research a wide range of contributions to showcase the various approaches and fields of application for action research. This text also illustrates how difficult it is to provide a clear-cut definition for action research.

The diversity of approaches could be explained by the early death of Kurt Lewin, one of the originators of action research, which prevented him from formulating a well-built description for this approach. This task was consequently left to fellow researchers, who reinterpreted Lewin's ideas and added their own views to the concept (Dickens \& Watkins, 1999). Baskerville and Wood-Harper (1998) also argue that a renewed interest in action research from the late 1970s onwards led researchers to propose new forms and applications for this methodology.

Hult and Lennung's (1980) description for action research is repeatedly mentioned in the literature (see DeLuca, Gallivan \& Kock, 2008; Lau, 1997; McKay \& Marshall, 2001; and Simon, 2000): 
Action research simultaneously assists in practical problem-solving and expands scientific knowledge, as well as enhances the competencies of the respective actors, being performed collaboratively in an immediate situation using data feedback in a cyclical process aiming at an increased understanding of a given social situation, primarily applicable for the understanding of change processes in social systems and undertaken within a mutually acceptable ethical framework (p. 247).

Perhaps the reason for the popularity of this description is the fact that Hult and Lennung (1980) built it after examining and analysing the action research literature available at the time. They identified the common constructive elements that described action research in many studies and integrated them into this definition.

A more recent attempt to formulate a working definition for action research was presented by Altrichter, Kemmis, McTaggart, and Zuber-Skerrit (2002), which is depicted in Table 3.

\section{If yours is a situation in which:}

- people reflect on and improve (or develop) their own work and their own situations

- by tightly inter-linking their reflection and action; and

- also making their experience public not only to other participants but also to other persons interested in and concerned about the work and the situation, i.e. their (public) theories and practices of the work and the situation.

And if yours is a situation in which there is increasingly:

- data-gathering by participants themselves (or with the help of others) in relation to their own questions;

- participation (in problem-posing and in answering questions) in decision-making;

- power-sharing and the relative suspension of hierarchical ways of working towards industrial democracy;

- collaboration among members of the group as a "critical community";

- self-reflection, self-evaluation and self-management by autonomous and responsible person and groups;

- learning progressively (and publicly) by doing and by making mistakes in a "self-reflective spiral" of planning, acting, observing, reflecting, replanning, etc.;

- reflection which supports the idea of the "(self-)reflective practitioner".

Then yours is a situation in which action research is occurring.

Table 3. Working definition of action research (source: Altrichter et al., 2002).

This definition was the result of a public discussion during the Brisbane International Symposium on Action Research in 1989. The first three points highlighted by this definition deal with the principles of action research in encouraging reflection, change, and dissemination of knowledge. The remaining points in the definition seem more descriptive of the methodology. 
The five most frequently-mentioned characteristics of action research will be discussed in the following paragraphs. These are: close to context, action and change, involvement, cyclical nature, and the ability to address practice and theory.

\subsubsection{Close to context}

Performing action research implies a close link with the context of the phenomena. The object of study is not separated from its environment intentionally. The context provides valuable insights about its situational and historical meaning, that will lead to a definition of the problem and eventually to its solution (Elden \& Chisholm, 1993; Hult \& Lennung, 1980).

Traditional research falls short in providing a viable solution to a problem, because it attempts to isolate a small number of factors about the situation and assumes that the rest remain constant (Hult \& Lennung, 1980; Zuber-Skerrit \& Perry, 2002). On the contrary, an action research approach will study the interconnections, interdependencies, and dynamics taking place within the situation of interest and how the introduced changes affect it (Baskerville, 1999; Dickens \& Watkins, 1999).

\subsubsection{Action and change}

McNiff (1988) argued that "action research means action, both of the system under consideration and of the people involved in the system" (p. 3). An aspect of the system is identified as the problem, and a planned action is taken which involves a degree of change in people's roles and responsibilities, as well as in the system where they are interacting (Baskerville \& Wood-Harper, 1998; Davison, Martinsons \& Kock, 2004).

A system can be any social setting, such as factories, schools, or business organisations, while people can be anyone playing a part in the system. This is precisely why Sommer and Sommer (2002) argue that action research is "value-driven" not "value-neutral," because it openly expresses the aim of improving a particular system.

\subsubsection{Involvement}

A third important characteristic is that action research allows direct involvement and collaboration between the researcher and the "problem owners" - as McKay and Marshall (2001) call the people being studied - in the research process and the solution to the problem (Baskerville \& Wood-Harper, 1996; Dickens \& Watkins, 1999; Sommer \& Sommer, 2001). McNiff (1988) expresses this by saying that action research "is 
research WITH, rather than research ON" (p. 4). In other words, the researcher is no longer researching a group or organisation and passing on the results; rather, they are assisting the problem owners in their inquiry. Both the researcher and the problem owners will depend on each other's skills, experience, and capabilities to carry out the inquiry (Hult \& Lennung, 1980).

Involving the problem owners in the inquiry achieves a better understanding of the context, as well as insights into their idiosyncrasies and a possible reasonable course of action (Baskerville \& Wood-Harper, 1996; Hult \& Lennung, 1980; Mumford, 2001). Including participants gives them a sense of ownership of the problem. They become more inclined to participate in the inquiry, and more committed to trying out possible solutions, than if they had merely to follow up the prescriptive advice of an external expert (Dickens \& Watkins, 1999). On the other hand, the researcher has opportunity to be involved, not simply be an observer or interpreter of the phenomena (Baskerville, Pries-Heje, 1999). They could contribute with an intellectual framework, a preunderstanding of the problem, and suggestions of intervention procedures to carry out the inquiry (McKay \& Marshall, 2001).

Hult \& Lennung (1980) clarify that involvement and collaboration do not mean there would not be a "rational" division of tasks among the problem owners and the researcher. For instance, Checkland and Holwell (2007) indicate that the researcher "must negotiate an exit from the situation" (p. 9) to engage in recording the learning that has taken place.

\subsubsection{Cyclical nature}

Action research is cyclical in nature. The action research team - that is, researcher and problem owners - will start by identifying a problem and the need for change in their system. The team will then propose and implement a solution for the particular problem. The intervention will be observed and its effects recorded, in order to evaluate and assess whether it has addressed the problem or whether the solution needs further refinement (Dickens \& Watkins, 1999; Hult \& Lennung, 1980). The participants will reflect on what happened, reformulate their thinking, and may even propose models or theories that explain the phenomena (Baskerville \& Wood-Harper, 1996; Lau, 1997). As Dickens and Watkins (1999) and Davison et al. (2004) indicate, solving the problem may take only one round of these steps; however, it is common for action research to take several iterations before reaching a satisfactory solution. 
The Susman and Evered (1978) action research cyclical model has frequently being referred to in the IS literature (Baskerville \& Wood-Harper, 1996; Simon, 2000; Rupino \& Dias, 2002). Figure 9 is a modified version of Susman and Evered's model by Baskerville and Wood-Harper (1996).

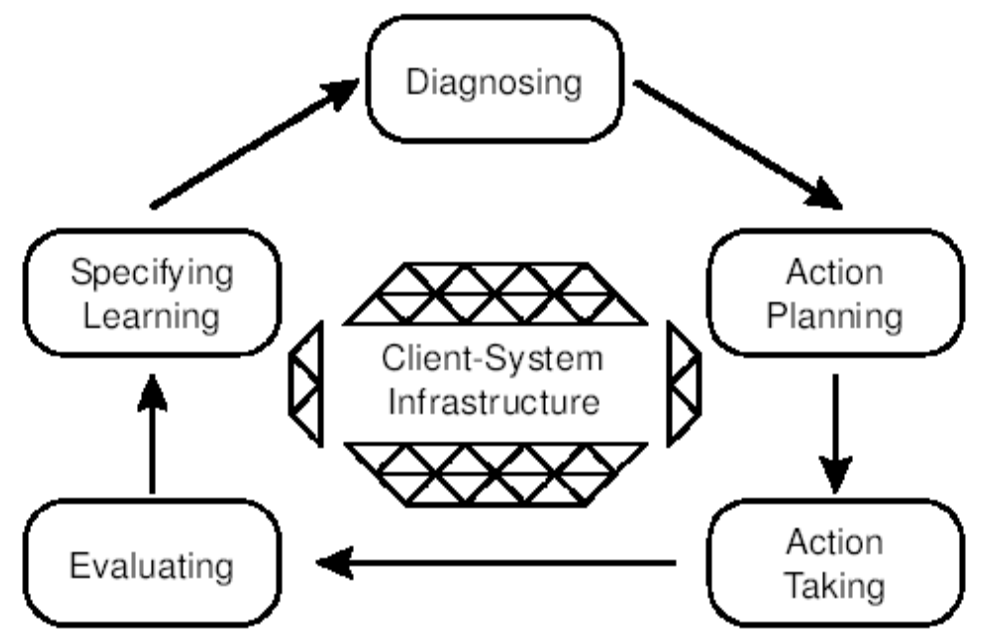

Figure 9. The action research cyclical model (source: Baskerville \& Wood-Harper, 1996).

The client-system infrastructure in the model refers to the specifications and conditions agreed by the researcher and the host organisation; it provides the rules of engagement in terms of research domain, authority and responsibilities, entry and exit considerations, legitimating actions, and allowance for the researcher to disseminate the learning that took place (Baskerville \& Wood-Harper, 1996; Rupino \& Dias, 2002). This model describes action research as a cycle of five stages: diagnosing, action planning, action taking, evaluating, and specifying learning. The following is a brief description of these five phases taken from Baskerville (1999):

- Diagnosing: Is the identification of the problem(s) as a whole and in its context. Here the team of researchers and practitioners will produce some theoretical assumptions about the organisation and the nature of the problem.

- Action planning: Based on the theoretical framework assumed in the first stage, the team will work together towards the design of a strategic plan to tackle the problem. The plan specifies the target and the approaches to be taken. 
- Action taking: The strategic plan is executed. In an attempt to solve the problem(s), researchers and practitioners will introduce some changes in the organisation according to the outlined strategic plan.

- Evaluating: The team will evaluate the outcome of the actions taken. The evaluation will determine if the theory that originated the strategic plan helped to solve the problem or not. With a positive outcome, the team has to assess whether the changes were the only reason for the success. In case of failure, the team can begin to correct the theoretical framework for the next cycle of action research.

- Specifying learning: This is an ongoing process in the cycle, but it becomes more relevant towards the end. Here, some reflection on the knowledge achieved through the exercise will take place, regardless of whether or not the action was successful. The knowledge gained can go to three audiences: the organisation, so that it can restructure its norms and procedures to reflect the new knowledge; the action research team, to use as the base for future action cycles; and the scientific community/general public, as a theoretical framework.

Even with an unsuccessful outcome, the new knowledge obtained will still be useful for the diagnosis phase of a new cycle in the search for a solution. Once the last stage has been completed, it could lead to another cycle of diagnosing, action planning, action taking, evaluating, and specifying learning, and so on. This will continue until there is a decision to stop the process.

It is worth pointing out that this action research cyclical model - and others proposed in the literature, for that matter - are simplified versions of the thinking and acting that occurs in the real world (McKay \& Marshall, 2001), and therefore each step should not be thought of as taking place in a neat, chronological order. As West and Stansfield (2001) comment: "we should take care not to read into the model any 'start' or 'finish' activities, or points of an action research study" (p. 256).

The cyclical process is as far as action research goes in describing how to carry out data collection, analysis, and validation. Action research gives freedom to the researcher and the participants to choose what type of diagnosis, model-building, deduction, data 
collection, data analysis, and evaluation techniques and methods they want to use for their inquiry, as long as such techniques are valid and reliable (Hult \& Lennung, 1980). The decision depends on the nature of the problem and how the researcher and problem owners wish to address this.

\subsubsection{Ability to address practice and theory}

Finally, as Hult and Lennung (1980) state, one key feature of action research is its ability to address a practical problem while at the same time generating knowledge. Researcher and problem owners will begin an action research inquiry with little understanding about a particular situation. It is through their observation, appreciation of what is happening, and reflection on the changes that have been implemented to solve the problem that knowledge takes place (Dickens \& Watkins, 1999; Lau, 1997).

Researchers and practitioners base the action framework on existing theoretical assumptions. At the end of each cycle this theoretical framework may be "reinforced, withdrawn, or modified" so that it better describes the real situation (Baskerville \& Pries-Heje, 1999). Each action cycle enables the evolution of knowledge that will benefit both practice and theory.

In the practical domain, action research proposes workable and sensible solutions to problems, increases collaboration between researchers and practitioners, and provides rich data about real-world problems (French \& Bell, 1999). Regarding knowledge, action research contributes to the understanding of the causes and dynamics of social problems, as well as proposing or refining theories/models of thought about social phenomena and social change, and detailed accounts of what occurred (Dickens \& Watkins, 1999; French \& Bell, 1999; Jönsson, 1991). The practical knowledge gained by the experience is made available to problem owners and their sponsors through reports. The theoretical knowledge will usually be expressed in dissertations or articles in academic journals (Mumford, 2001; Zuber-Skerrit \& Perry, 2002).

Based on the premise that the action research works towards improving a practical situation, while at the same time aims to generate new knowledge, Kock, Avison, Baskerville, Myers, Wood-Harper (1999), and Narayanaswamy and Grover (2007) comment that action research serves two masters. McKay and Marshall (2001) are of the view that many action research models illustrate only the cyclical approach of action research and share little guidance on the aspect of reporting knowledge. 
A similar concern was shared by Zuber-Skerrit and Perry (2002), who noticed that postgraduate students were finding it hard to distinguish between the collaborative project work and their individual thesis work. These authors made a distinction between the two activities - which they called core action research project and thesis action research project - and proposed the cyclical model of Figure 10 to illustrate the interactions.

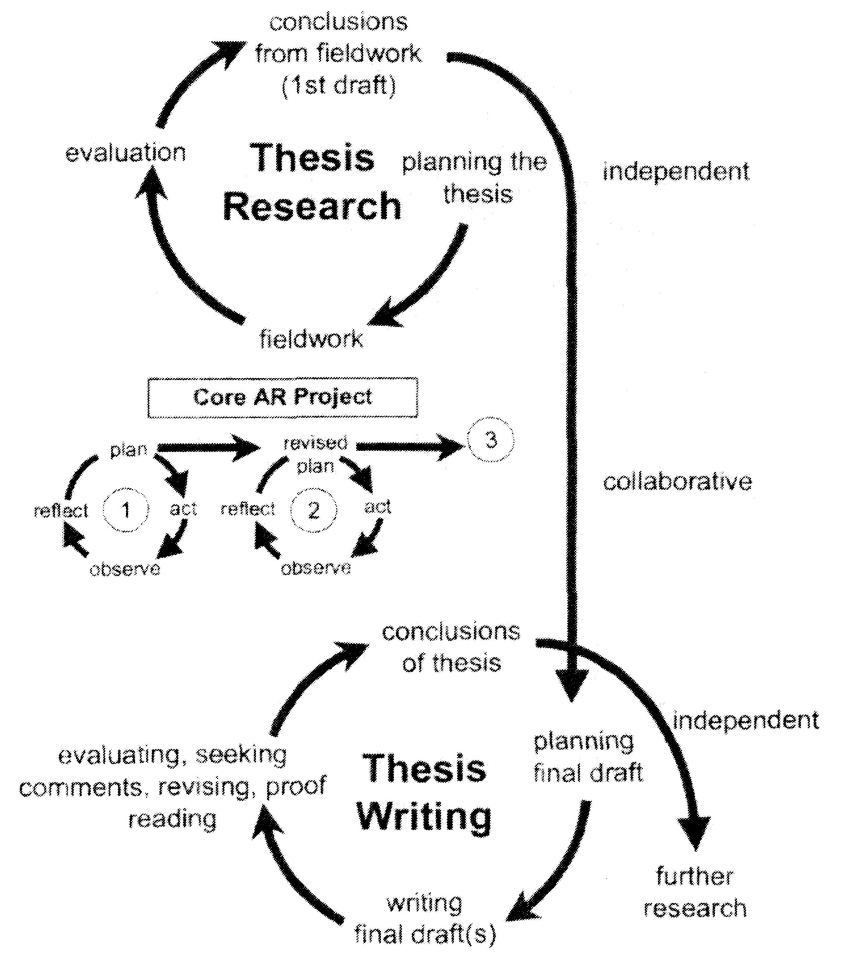

Figure 10. The core action research and thesis action research cycle (source: Zuber-Skerritt \& Perry, 2002)

\subsubsection{Action research in Information Systems (IS)}

During the 1980s and 1990s the IS community engaged in debate about accepting other research paradigms apart from the positivist stand (Benville \& Landry, 1992; Lau, 1997; Orlikowski \& Baroudi, 1991). This was triggered by a growing interest in exploring the organisational, behavioural, and social consequences of introducing technology and information systems in the workplace rather than merely focusing on the technical component (Baskerville \& Wood-Harper, 1996; Lau, 1997).

Furthermore, during the 2007 European Conference of Information Systems it was acknowledged that there was a shortage of IS research relevant to practitioners, and the question of how to address this was raised (DeLuca et al. 2008). These discussions also involved the use of methodologies that could accord with new philosophical stances 
enabling the carrying out of more research in natural business settings, action research being one of these (DeLuca et al., 2008; Galliers, 1991).

Baskerville and Wood-Harper (1996) consider that action research fits nicely in the IS discipline given that "IS is a highly applied field, almost vocational in nature" (p. 235) and action research is a hands-on methodology. For instance, Davison (2001) found the cyclical structure of action research useful when he introduced a Group Support Systems (GSS) to a management skills training course for the Hong Kong Police Department. Each training session embodied a cycle of planning, executing, observing, and reflecting on the experience of using GSS for group discussion, deliberation, and decision-making. By this method the quality of subsequent sessions employing GSS improved. The project addressed the practical problem of encouraging police officers to participate by temporarily fading cultural barriers to communication through GSS, while looking at theories about collaborative learning and technology-supported learning.

Yoong and Gallupe (2001), on the other hand, found action learning - a variant of action research - useful when exploring new research themes. They were interested in learning how facilitators of conventional meetings would make the transition to facilitate face-to-face meetings mediated by GSS. Since there was no prior experience in training facilitators for this type of technology, action learning was the appropriate methodological framework to encourage learning in collaborative groups. Through ongoing action and reflection activities among the participants, key processes were identified that helped in gaining the skills and insights required to manage and support electronic meetings. The theoretical output of the study was a model that depicted the learning processes and experiences by the facilitators/participants during their training time. The practical outputs were facilitators who mastered a new skill, and a sound training programme for preparing future facilitators.

Cockburn-Wootten, Henderson and Rix's (2005) action research project caught my interest because it examined developing a university department's website as an internal and external communication tool, which is similar to what occurred in this study. They found action research a suitable methodology, given the organic and dynamic structure of the department and the task at hand. Action research was also selected because of its cyclical nature which facilitated feedback loops during the design of the website. Action research allowed the researchers to consider the social context of the project, an aspect 
that had a major impact on the study's outcome. Unfortunately the website was not completed by the end of the research project, but the project generated useful knowledge for both practitioners and researchers. The participants were made aware of their present organisational culture and how it hindered them reaching their goal. The knowledge angle of the project highlighted aspects to take into account when implementing changes in an organisation, such as staff knowledge of the technology, issues of power, and credibility.

Some other areas in IS where action research has worked well are in the development and implementation of IT systems (Chiasson \& Dexter, 2001), the reorganisation of information systems (Jönsson, 1991; Simon, 2000), and the design of socio-technical systems (Mumford, 2001). In particular, Baskerville and Wood-Harper (1996) regard action research as an appropriate approach for studying the effects of changes in systems development methodologies. Other research methods such as statistical modelling or laboratory experiments do not consider the complex, multivariate context of the study, which may have some influence on the results.

Lau (1997) conducted a review of the published literature on action research in IS between 1970 and 1995 to observe how action research has been performed in the IS field. At the beginning of this period most of the articles: 1) failed to declare and explain the use of action research, 2) were unclear on the methodology used, or 3) used a definition regarded as debatable. However, by the end of the period a small but increasing number of studies using action research started to appear in IS journals and conference proceedings (Baskerville \& Wood-Harper, 1998; Lau, 1997). This suggested a growing awareness of action research as a promising methodology for IS inquiry.

The small number of studies employing action research in IS by the late 1990s and early 2000s confirmed that this methodology needed to overcome further challenges, among them acceptance by researcher and mainstream IS journals, and the diffusion of studies following action research methodology (Lau, 1999). In particular, Lau (1999) reported that the absence of a framework for action research with an IS flavour was one important reason proposed during the International Federation for Information Processing (IFIP) Conference in 1997 to explain why action research had not taken off in the IS field.

The absence of detailed guidelines is due to the fact that each situation is unique, therefore each project will be designed according to its individual needs (Jönsson, 
1991). Consequently, it is not possible - and besides, it would be against the very nature of action research (Checkland, 1981 cited in Davison, 2001) - to draft rigid procedures for performance (Avison, Baskerville \& Myers, 2001). Nevertheless, Avison, Lau, Myers and Nielsen (1999), and McKay and Marshall (2001) are of the view that an action research framework with some overarching points will not only guide the novice action researcher on how to proceed with this "new" methodology in IS, but will also assist in assessing the quality and rigour of such studies.

Responding to the encouragement to adopt and advance the rigour of action research, accomplished IS action researchers have written articles providing advice and some criteria on how to go about embarking on an action research inquiry. Among them are Kock, Baker, McQueen and Rouse (1996), who discuss choosing and negotiating with organisations for an appropriate action research topic; Lau's (1999) action research framework which collates and refines some dispersed criteria proposed by the IS literature; and Avison, Bakerville and Myers (2001), who propose controlling structures to ensure balance between practice and research.

Lately, Narayanaswamy and Grover (2007) have followed the steps of Lau (1999) and developed an updated framework to guide action research processes. Their comprehensive framework covers six dimensions (study design, control issues, structure, methodology, application, and specifying learning) and was a result of encompassing the current action research literature.

Narayanaswamy and Grover (2007) found that there has been an increase of action research studies in the literature, thus showing a growing acceptance of this methodology in the IS domain in recent years. They also observe that, although the more current studies are incorporating most of the elements of their framework, there are still gaps in terms of explaining aspects of rigour, authority, and ethical issues.

The recent Information Systems Action Research (Kock, 2007) features contributions from IS action researchers on key topics that need further discussion. For instance, Checkland and Holwell (2007) introduce the "recoverability" criterion to validate action research studies. On the other hand, Avison, Baskerville, and Myers (2007) elaborate on the concept of power in action research projects. According to its editor, this text "represents the state-of-the-art of IS action research today" (Kock 2007:xxii). 


\subsubsection{Variants of action research in the IS literature}

Another issue discussed in the IS literature was the different action research variants or streams that are followed by the IS community. Lau (1999) identifies four action research variants in his framework: action research stream, which focuses on changing practice; action science, useful for conflict resolution; participatory action research, for participant collaboration inquiries; and action learning, which focuses on experiential learning.

Baskerville and Wood-Harper (1998), on the other hand, propose more inclusive boundaries for action research in IS, which would suit IS research better than approaches (which they consider exclusive) defined in other social sciences. They argued that IS research tends to be highly empirical - where theoretical frameworks, hypotheses and/or algorithms are grounded in practice - and therefore action research in IS should accept practitioner-oriented forms like prototyping, process consultation, and clinical field work ${ }^{9}$. By doing this, readers would have access to knowledge that before was mistakenly perceived as not emerging from rigorous methodologies. At the same time this would allow researchers to have stronger claims of validity and generalisability for their work.

Baskerville and Wood-Harper (1998) identify ten forms of action research: canonical action research, IS prototyping, soft systems, action science, participant observation, action learning, multiview, ETHICS, clinical field work, and process consulting. They then proceed to describe and compare these forms of action research based on four parameters: process model, structure, researcher involvement, and primary goal. Table 4 summarises their typology.

\footnotetext{
${ }^{9}$ As a note of caution, Baskerville and Wood-Harper (1998) acknowledge that not all the work from these new forms - especially practitioner prototyping - would fit the action research methodology.
} 


\begin{tabular}{|c|c|c|c|c|}
\hline $\begin{array}{l}\text { Form of action } \\
\text { research in IS }\end{array}$ & Process model & Structure & $\begin{array}{l}\text { Researcher } \\
\text { involvement }\end{array}$ & Primary goal \\
\hline $\begin{array}{l}\text { "Canonical" action } \\
\text { research }\end{array}$ & Iterative & Rigorous & Collaborator & $\begin{array}{l}\text { Organisational development } \\
\text { or scientific knowledge }\end{array}$ \\
\hline IS Prototyping & Iterative & Rigorous & Collaborator or facilitator & Systems design \\
\hline Soft Systems & Iterative & Fluid & Facilitator & $\begin{array}{l}\text { Organisational development } \\
\text { or systems design }\end{array}$ \\
\hline Action Science & Reflective & Fluid & Facilitator & $\begin{array}{l}\text { Organisational development } \\
\text { or scientific knowledge }\end{array}$ \\
\hline $\begin{array}{l}\text { Participant } \\
\text { Observation }\end{array}$ & Reflective & Fluid & Expert & Scientific knowledge \\
\hline Action Learning & Reflective & Fluid & Expert & Training/education \\
\hline Multiview & Linear & Rigorous & $\begin{array}{l}\text { Collaborator, facilitator } \\
\text { or expert }\end{array}$ & Systems design \\
\hline ETHICS & Linear & Rigorous & Facilitator & $\begin{array}{l}\text { Organisational development } \\
\text { or systems design }\end{array}$ \\
\hline $\begin{array}{l}\text { Clinical Field } \\
\text { Work }\end{array}$ & Linear & Fluid & Facilitator & $\begin{array}{l}\text { Organisational development } \\
\text { or scientific knowledge }\end{array}$ \\
\hline $\begin{array}{l}\text { Process } \\
\text { consultation }\end{array}$ & Linear & Rigorous & Expert & Organisational development \\
\hline
\end{tabular}

Table 4. Forms of action research in IS (source: Baskerville \& Wood-Harper, 1998).

A process model depicts how the study is carried out. It can be one of three possibilities: a) iterative, which is a repeating sequence of activities to identify and solve the problem in a cyclical manner; b) reflective, which is also iterative but focuses mainly on discovering differences between the theory that the researcher claims to be following and the theory that emerges from action; and c) linear, which is carrying out a sequence of activities just once, without iteration.

According to Baskerville and Wood-Harper (1998), there are two main structures in action research: rigorous and fluid. Rigorous structures have defined stages, steps, or activities to follow in a sequence or cycle. Fluid structures not only define such activities loosely but allow flexibility about how and when to undertake them.

In action research studies, researchers play a more participatory role. Depending on the type of research and/or their personal choice, the researcher's involvement can be that of: a) collaborator, where they are part of the team as an equal, sharing tasks and working alongside participants towards a solution to the problem; b) facilitator, where they not only help in facilitating the group, but are also considered an expert to whom participants can turn for advice, technical knowledge, or opinions during decision- 
making; and c) expert, where they stand out from the rest of the participants and have a direct responsibility for solving the problem.

Baskerville and Wood-Harper (1998) argued that, although each form of action research could focus on many goals, usually one primary goal dominates. Four common goals in action research studies are: a) organisational development, which looks at improving the social conditions of an organisation in aspects such as morale and information flows; $b$ ) systems design, which focuses on creating or improving the organisational systems; c) scientific knowledge, which looks at making contributions to the scientific literature; and d) training/education, which involves participants learning from the study.

Baskerville and Wood-Harper's proposition of including practitioner-oriented forms as action research approaches is not entirely accepted by the IS community. For instance, McKay and Marshall (2001) believe that soft systems methodology, prototyping, and ETHICS are problem-solving processes that - with the exception of prototyping - have been employed in action research methodology. Since these forms have been associated with action research they have acquired some features of action research, hence the confusion. McKay and Marshall's (2001) main argument is that none of these forms explicitly address the research cycle of action research. This issue is the subject of ongoing debate.

\subsubsection{Rationale for using action research in this study}

The main features of action research explored in this Section corroborate the consistency of this methodology with the advocacy/participatory research paradigm, which is my philosophical stance for the present study.

Action research enables the study of knowledge sharing in clusters in its contextual and historical dimensions. As it will be discussed in Chapter 5, the New Zealand Government, the Wellington Regional Council, and local private firms worked towards stimulating the development of clusters in the region. The development programmes they implemented to promote innovation, the support these entities provided (i.e. financial, expertise, logistics, and technical), and the way they interacted with the clusters influenced the setting of this study.

Another reason for selecting action research methodology is the high level of involvement that both researcher and participants experience in the research inquiry. 
Through collaborative work, action research empowers other participants with a voice in the development of theoretical frameworks (Baskerville, 1999). As a researcher, I can contribute towards addressing the practical problem.

In addition, action research goes beyond interpreting what the clusters are experiencing to achieve good knowledge transfer mechanisms and proposes some interventions, thus encouraging change. Finally, action research guided my goal of adding to the knowledge in the IS field.

\subsection{The study's action research design}

The previous Section provided a detailed account of action research and its present situation in the IS discipline. I have also included evidence that this methodology is in line with my philosophical research stance and will be appropriate for exploring my research questions.

Avison, Lau, Myers and Nielsen (1999) explain the importance in action research studies of making clear one's research aim, theory, and methodology; failing to do so may risk the study being perceived as a mere consulting job in a real-life situation. An even worse scenario is what Kock (1997) experienced during an exploratory action research study conducted in a Brazilian company, where the lack of a clear research framework at the start of the study resulted not only in insufficient data to support some findings and conclusions, but also in an excessive collection of irrelevant information.

This Section is based on the guidelines provided by Creswell (2003) and Morse (1998) for qualitative inquiry in general, and on Lau's (1999) and Baskerville's (1999) action research frameworks in particular. Suggestions from recent papers addressing action research design were also considered. The following sub-sections describe how data collection, analysis, and reporting were carried out in this study.

\subsubsection{Canonical action research as the selected stream}

The previous section explained how action research is in fact the generic name for a collection of methodologies. Broadly speaking, the IS literature acknowledges four types: canonical action research, action science, participatory action research, and action learning. 
Lau (1997) indicates that the choice of the type of action research would depend on the focus of the study. He argues that canonical action research fits better when exploring change in practice, action science is useful for conflict resolution, participatory action research works in situations of participant collaboration, and action learning is appropriate for experiential learning.

Based on Lau's (1997) argument, this study followed canonical action research since one of its main purposes was to explore how ICT can support or enhance knowledge transfer in the cluster's project bidding process. This implied that cluster members ${ }^{10}$ and the researcher worked together to create a new information system to better support knowledge sharing.

Participatory action research could have been another possible alternative, because cluster members are working as collaborators on an equal basis with the researcher. I rejected this option because it puts too much emphasis on participants' involvement in all the stages of the research process in terms of data gathering, analysis, and report writing (Bell, Giordano \& Putz, 2002). Most of the cluster members I worked with are service providers, and hence have constraints as to how much time they could grant to a research project of this magnitude.

I followed Susman and Evered's (1978) cyclical model (see Figure 9 in the previous Section), which is in line with canonical action research (Davison et al., 2004). I also considered Zuber-Skerrit and Perry's (2002) model (see Figure 10 in the previous Section) as a reminder of the actions needed in both practice and research outputs.

\subsubsection{The research site}

When I began this study in 2003 there were eight clusters in the Wellington Regional Council's cluster programme (Harvey \& Gray, 2003): Natural Hazards New Zealand, New Zealand Earthquake Engineering, Creative Capital, Creative Manufacturing, ICT, Tertiary Education, Maori Consulting, and Pacific Island Consulting. These clusters can fit Chisholm and Elden's (1993) description of tightly-organised systems, as they have outlined membership boundaries, they share a common understanding of the purpose

\footnotetext{
${ }^{10}$ Strictly speaking, a cluster member is an organisation, which may be a one-person company or a multiemployee company, but not an individual. Each organisation assigns one of its staff members to represent it in cluster activities. For the purpose of this study I am assuming that a cluster member is the individual that represents their company.
} 
and goals of clustering, their roles are clearly defined, and they have some policies and work procedures in place.

I approached Natural Hazards New Zealand (NHNZ) and Earthquake Engineering New Zealand (EENZ) and proposed to their Facilitator and Co-Chairs the possibility of working with them on a project where knowledge transfer was the underlying theme. We discussed the opportunity for me to generate knowledge on this topic, at the same time supporting the clusters in developing an information system to improve knowledge transfer among cluster members. Both clusters were interested in exploring this proposal further, as they found it highly relevant to their business activities.

One of the reasons I chose to approach these two clusters was the fact that I already knew something about them. In 2001 I interviewed the Co-Chairs of these two clusters as part of a report for an Honours paper exploring network firms and the role of ICT. This assignment triggered my interest in the topic, and I also found the Co-Chairs very supportive of any initiative that could benefit their clusters.

In 2002, Dr Pak Yoong and I were awarded a research grant to study knowledgeintensive organisations. Since clusters fitted the grant's criteria we decided to look at the factors affecting knowledge sharing in this type of networked organisation (Molina \& Yoong, 2003). Although representatives of seven clusters in the Wellington region took part in the study, most came from NHNZ and EENZ. This gave me the opportunity to meet more people from these two clusters.

In April 2003, at the Industry New Zealand (now known as New Zealand Trade and Enterprise) Cluster Development Workshop, I met the Facilitator who supports both clusters. Being a natural "net-broker" 11 he was interested in linking my research with the clusters he was looking after, as he perceived the possibility of synergy.

\subsubsection{Entry to the site}

Executing action research in an organisation requires the researcher to negotiate entry. To achieve this, Kock et al. (1996) pointed out that the researcher's and the organisation's views need to be considered: on the one hand, the researcher must see

\footnotetext{
${ }^{11}$ Fanke \& Hickmann (1999) used this term to describe a person acting as a facilitator in a network of firms. The net-broker is a dynamic person who is involved in bringing companies together to set up strategic partnerships, organise network activities, promote cooperation, and be constantly on the look-out for potential business opportunities.
} 
that the study would give them the opportunity to enhance knowledge; on the other hand, the organisation must see that the study is relevant to them, and that by taking part in it they would gain the opportunity to improve. Both views have to be reconciled and the action research study tailored accordingly.

The experience I gained working on the study of knowledge sharing in clusters (Molina \& Yoong, 2003) made me realise that that my appreciation of clusters was going to be limited if I could be only an outsider looking in through a window. I needed to get inside and be part of the cluster, at least to some extent. Fortunately this previous study allowed me to meet some key people such as cluster Co-Chairs, the Facilitator, and officials from Positively Wellington Business (the economic development agency that supported clusters within the region), whom I approached to discuss the possibility of involvement with the clusters.

Conversations were held with cluster stakeholders, negotiating the research topic and my entry to the research site. I was also invited to attend both clusters' quarterly meetings early in the process. My understanding of the cluster context improved, which allowed me to be more specific in my research questions, purpose, and deciding what the practical component should be. In addition, these talks increased my confidence that we could successfully accommodate the clusters' needs in order to make the study relevant for them, while at the same time generating some theories from the role of ICT in knowledge transfer.

While defining my $\mathrm{PhD}$ research proposal in 2003, I worked on a voluntary basis for the Facilitator of Earthquake Engineering NZ (EENZ) and Natural Hazards NZ (NHNZ). The tasks I performed were administrative in nature, such as photocopying, searching for contact details, and preparing PowerPoint presentations. Working as a volunteer for the cluster Facilitator provided me with valuable insights into how this study could be conducted and how to refine my research proposal.

As part of the entry process, Mumford (2001) and Baskerville (1999) recommend writing a document that outlines the research domain, agreed processes, responsibilities, objectives, and outputs of the study that were reached during the negotiation. Mumford (2001) calls such a piece an "action document," while Baskerville (1999) considers it part of the "client-system infrastructure." This document contributes to reducing misunderstandings and false expectations about the action research study. Baskerville 
(1999) also indicates that it is beneficial to express in the document the researcher's intent to disseminate the learning that took place during the research.

I agree with the above advice and therefore prepared two letters of agreement (my versions of an action document) between the clusters and me: one scoping my involvement as a volunteer and the second defining the first stages of my $\mathrm{PhD}$ study. Both letters covered the points suggested by Baskerville (1999) and Mumford (2001).

Despite the fact that there is a written document to support the action research, Mumford (2001) advocates a flexible stance. Her view is that in most cases research does not go as planned, and therefore the "action document" should be viewed as a point of reference from which both researcher and participants may discuss and justify changes made to the initial plan over time.

\subsubsection{The role of the researcher}

Chisholm and Elden (1993) indicate that the role assumed by the researcher in the action research inquiry could range from a dominant stance to a more open and collaborative one. In the dominant position, the researcher keeps control of the key aspects of the research process, such as its design, data collection, analysis, and interpretation. However, the authors warn that the outcomes of a highly controlled inquiry could depict a bias towards the researcher's perceptions of the reality, thus underplaying participants' knowledge and opinions. In my opinion, this contradicts the nature of action research, which is to give participants the opportunity to shape the inquiry and tell their story.

At the other end of the scale, the researcher in a highly collaborative role will work with participants in all aspects of the inquiry. Although participation is something to pursue in action research, I believe that by giving too much control to participants the project could get sidetracked into solving the practical problem and not enhancing knowledge. Baskerville (2001) calls this situation "second-degree outcome failure."

My position as a researcher for this study fell somewhere between Chisholm's and Elden's (1993) two extremes. As a researcher I was responsible for fulfilling the theoretical aspect of this project, and therefore I took the initiative in designing the research process and ensuring that all the pertinent steps had been taken. Conversely, working collaboratively with cluster members increased the value of this study, not only 
because their voices and opinions were heard, but also because they had a better idea of what worked in the context of the study. Cluster members are very knowledgeable about their field and business, so it was sensible to take into account their suggestions and views on the project.

As pointed out by Davison et al. (2004), the level of involvement by the participants and me varied during the various stages of the action cycle of this study: I drove the diagnostic stage, the planning and development stages of the study became highly collaborative between both parties, and finally the evaluation and specifying learning stages once again became my responsibility.

Despite declaring my position with regard to the level of control and collaboration, I am falling short in stating which part I played in the study. The categories proposed by Baskerville and Wood-Harper (1998) for the action researcher role are a practical way to look at this matter. As already explained in Section 4.2, these authors identify three roles for the action researcher: collaborator, facilitator, and expert.

My role was consistent with Baskerville's and Wood-Harper's (1998) proposition that a canonical action research calls for the researcher as a collaborator (see Table 4), especially during the planning and development stages. This is due partly to my personal belief that participants should play an active role in designing solutions to their own problems. Besides, it would have been pretentious on my part to walk into a cluster and advise it on a course of action, given my limited experience in research, cluster activity, and project management.

While working as a volunteer for the cluster Facilitator, I noticed that the clusters tend to form small groups to work on a particular task and then report their progress back to the rest of the cluster. The practical component of this study was carried out by two of such task groups, which I volunteered to join: the Website Task Group and the Content Task Group.

Within the task groups I found myself playing the role of project coordinator. Some activities that I carried out included organising the task group meetings, sharing ideas and suggestions during the meetings, helping conceptualising the system, liaising with the website developer, and writing the draft and final reports. Nevertheless, I made sure that the cluster members made the decisions on how to advance the project: 
It was good we had a brief meeting because there were some questions that I could answer and the developer could carry on with his work. However, there were a few key questions that need the consensus by the rest of the team and therefore [web developer] will have to wait until the Task Group can meet with him. (Email Researcher, 26 July 2004).

The researcher is also committed to continuously reflect on the actions taken and their outcomes (Checkland \& Holwell, 2007; McKay \& Marshall, 2001). This involves making sense of how the experience is progressing, how the declared framework and methodology are performing, and what the emerging themes are. Reflection leads to the formulation of new interventions to solve the practical problem and to the building of theory.

Finally, I am clear that reporting knowledge to the scientific community is entirely my responsibility (McKay \& Marshall, 2001; Zuber-Skerritt \& Perry, 2002). Therefore I took the initiative in designing the study, collecting and analysing data, and interpreting the findings. Collaboration and suggestions to complete these activities were requested from cluster members and other stakeholders (such as cluster Facilitator, Positively Wellington Business staff, and research supervisors) and their feedback incorporated.

\subsubsection{The role of the participants}

Participants are valuable assets for action research as they have a deep understanding of their system, have a feeling on how others in the group perceive the situation, and even have an idea of how to accomplish the task (Chisholm \& Elden, 1993; West \& Stansfield, 2001). Participants in action research studies are no longer passive recipients of the research process. They can contribute with decisions about the study, such as the wording of questions in a survey, who should be interviewed, how the results will be analysed, and what actions will be taken (Sommer \& Sommer, 2001).

Similar to the researcher's stance, the participants' level of involvement in a study could vary from high control to collaboration (Chisholm \& Elden, 1993; Lau, 1999). When both factions are combined, involvement is inversely proportional. In other words, if a researcher has high control then participants' involvement is minimal, but if the researcher exercises less control then participants would have more influence in the study.

Lau (1999) indicated that the participants' role would depend on how the action research inquiry has been framed. He also pointed out that the participants' role should 
be "authentic, appropriate, and effective in helping to solve the problem and learning from the experience" (p. 168).

For this study, cluster members played an active role in the planning and execution stages of the action research cycle. They formed task groups to address their practical problems. They collaborated in the research cycle by providing suggestions, feedback, and ideas, but were not highly involved in the development and reporting of theoretical knowledge.

To guarantee that the cluster's views were considered in the development of theory, I suggested to the cluster the setting up of a Cluster Advisor during my negotiations for entry. This cluster member would mediate between the cluster and me. I submitted my findings to this person for his consideration and input.

\subsubsection{Data collection procedures}

Data collection is concerned with gathering information that will be used to address the research questions and to solve the clusters' practical problems. Since my advocacy/participatory stance about knowledge states that the subjects of my study cannot be isolated from their contextual and historical dimensions, one of the kinds of data I collected assisted me in describing the social, economic, and political factors that influenced the clusters and our performance in answering the research questions. The selection of genre analysis as the theoretical framework for this study also helped me focus on the kinds of data I needed to collect.

Another data type I collected was what Hult and Lennung (1980) and Jönsson (1991) call the changes in the system caused by the interventions of action research. Implementing change generates data that the action research team needs to collect in order to gain understanding of how the system is responding and to decide if another action cycle is required. This is why Elden and Chisholm (1993) consider that it is imperative to have a systematic collection of data over time concerning actions taken and their respective consequences.

Having identified the kinds of data this study required to ensure the development of knowledge and the improvement of practice, I then focused on the techniques to collect these data. McNiff (1988) argues that data collection in action research is an "eclectic" exercise, where the researcher will use any data collection technique that seems 
appropriate for the particular situation. It was helpful to come across Creswell's (2003) classification of data collection techniques for qualitative methodologies. He divides data collection techniques into four groups: observations, interviews, documents, and audiovisual materials.

Observations are notes taken by the researcher at the research site. These notes can include detailed descriptions of events, behaviour of individuals, comments, and any other activity that is pertinent (Creswell, 2003).

Interviews allow the researcher to interact with the interviewees, ask them specific questions, and have the opportunity to receive instant feedback on the enquiry (McNiff, 1988). Interviews can be conducted face-to-face, by telephone, or mediated by computers (e.g. videoconferencing). This technique can take place on a one-on-one basis or in a small group of people, such as a focus group. Less structured interviews with open-ended questions rather than interviews with pre-set questions will tend to encourage interviewees to express their own views and opinions about an issue (Creswell, 2003).

A third form of data collection is through documents. Documents can be public, such as newspapers, newsletters, meetings minutes, official reports, and journal publications; or private, as in the case of personal journals, diaries, letters, and e-mails (Creswell, 2003).

Finally, audiovisual materials are creative sources of data. Some examples are photographs, videotapes, sound records, and computer software (Creswell, 2003; McNiff, 1988).

Table 5 lists some advantages and disadvantages for each of these four techniques of data collection. As a novice action researcher, I needed to be aware of such strengths and weaknesses to assess the technique most appropriate for a particular situation, and how to complement each technique to collect the best data possible. 


\begin{tabular}{|c|c|c|}
\hline $\begin{array}{c}\text { Type of } \\
\text { data } \\
\text { collection }\end{array}$ & Advantages & Disadvantages \\
\hline Observations & $\begin{array}{l}\text { - First-hand collection of data. } \\
\text { - Information is collected as it unfolds. } \\
\text { - Allows the recording of subtle } \\
\text { comments, gestures, hunches, etc. }\end{array}$ & $\begin{array}{l}\text { - Time-consuming exercise. } \\
\text { - May misinterpret what was seen or } \\
\text { heard due to bad attending and } \\
\text { observing skills. } \\
\text { - Difficulty in quantifying data. }\end{array}$ \\
\hline Interviews & $\begin{array}{l}\text { Useful when researcher cannot } \\
\text { observe participants directly. } \\
\text { - Collects data directly from } \\
\text { participants. } \\
\text { - Researcher has some control over } \\
\text { what data s/he is collecting. } \\
\text { - Interactive, so it allows instant } \\
\text { feedback and clarification of ideas. } \\
\text { - Collects non-verbal communication. }\end{array}$ & $\begin{array}{l}\text { - Time-consuming exercise. } \\
\text { - Participants provide information from } \\
\text { their point of view. } \\
\text { - Interviews are not natural. } \\
\text { - Interviewee may not provide honest } \\
\text { replies due to the presence of } \\
\text { researcher. }\end{array}$ \\
\hline Documents & $\begin{array}{l}\text { - Documents can be viewed at any } \\
\text { time. } \\
\text { - Non-intrusive source of information. } \\
\text { - It is not "raw"; that is, some thought } \\
\text { and attention has gone into preparing } \\
\text { the document. } \\
\text { - No need to transcribe. } \\
\text { - Written evidence to support or } \\
\text { contradict claims made during } \\
\text { interviews. }\end{array}$ & $\begin{array}{l}\text { - May be difficult to obtain. } \\
\text { - Document may not be authentic or } \\
\text { - Occurate. } \\
\text { - imme-way information, hence no } \\
\text { - Material may be incomplete. } \\
\text { - Lack of non-verbal communication. } \\
\text { - Diaries are subjective. }\end{array}$ \\
\hline $\begin{array}{c}\text { Audiovisual } \\
\text { materials }\end{array}$ & $\begin{array}{l}\text { Most are not intrusive techniques to } \\
\text { collect data (an exception could be } \\
\text { videotapes). } \\
\text { - Audio and video records are accurate. } \\
\text { - A way for participants to share their } \\
\text { reality. } \\
\text { - Effective way to catch the attention of } \\
\text { viewers. }\end{array}$ & $\begin{array}{l}\text { - Some of them may be difficult to } \\
\text { interpret. } \\
\text { - Presence of the recording appliance } \\
\text { may inhibit individuals. } \\
\text { - Material may be confidential or } \\
\text { difficult to access. } \\
\text { - Some of these are awkward to use and } \\
\text { expensive (e.g. videotapes, software). }\end{array}$ \\
\hline
\end{tabular}

Table 5. Data collection techniques for qualitative methods (sources: Creswell, 2003; McNiff, 1988).

Once the types of data collection techniques for qualitative methods have been identified, the next step is to select which will be used to collect data for this study. I looked at the literature for ideas on how other action researchers - in particular those who have worked on developing information systems - have collected their data.

Before starting to collect data, Morse (1998) believes that the researcher should spend some time learning "who's who," the routines, and the formal and informal rules of the site. This way, the researcher has a better understanding of the roles and relationships amongst participants, allowing them to identify the appropriate informants for data collection. For Morse (1998), good informants have the knowledge and experiences required for the study, are able to reflect and articulate ideas, are available, and are willing to participate in the study. 
Kock (1997) argues that, although observational notes may be one of the most important data collection techniques for action research, it is wrong to think that data should be gathered only in this way. By relying excessively on observational notes, the researcher may give explanations and conclusions about the phenomena that are in tune with their personal preference rather than with what is really happening. To overcome this risk, Kock (1997) recommends combining observational notes with other data collection techniques that do not have the researcher's biases, such as participants' interviews and questionnaires.

Braun (2003a) and McNiff (1988) support the use of journals (or learning diaries) for data collection in action research. The researcher and participants can keep a journal to register their own perspective, feelings about, and experiences of the project. Keeping a learning diary encourages reflection on the actions taken. As in the case of observational notes, the data collected from journals should be handled with caution, as overgeneralisation thinking could distort conclusions (Braun, 2003a). It is significant to note that Wellington (2002) found it difficult to encourage participants to write diaries because this was time-consuming for them.

West and Stansfield (2001), on the other hand, advise researchers to keep track of what is occurring during the project, in particular ideas, changes in practice, and attitudes that emerge over time. In their respective PhDs West and Stansfield (2001) tape-recorded and transcribed meetings with their participants.

The search of the literature provided a better understanding of what data collection techniques are available and how some of them have been employed in previous action research studies. Based on this information I opted for the following combination of data collection to answer the research questions:

I made observational notes on what took place during the cluster meetings I attended. I was especially alert in noting what happened during the Task Groups' meetings while working on the project. This ensured that lessons were documented and reflected upon throughout the project rather than only at the end. By doing this, there was a record of how my own thinking and those of the participants was shaped over time. I also kept a personal journal during this project, to complement my observational notes with my own thoughts and reflections about the experience of carrying out this study. 
Given the troubles encountered by Wellington (2002), I decided not to ask participants to keep a journal. Like the engineers and designers in Wellington's (2002) study, cluster members are very pressed for time.

I used semi-structured interviews to collect participants' views on specific topics. I also interviewed the Facilitator and the official from PWB to enrich the contextual setting of the study. I tape-recorded each interview - subject to permission granted by the participant - to guarantee that all the interviewees' comments and ideas were captured accurately, and transcribed each shortly afterwards. The first interview instrument was reviewed and approved by the Human Ethics Committee of the Faculty of Commerce and Administration, Victoria University of Wellington. Four other interview instruments were used at different points in the study. All the interview instruments are included in Appendix A.

Finally I collected cluster agendas and meeting minutes, task group reports, and any other document produced by the cluster or its stakeholder which was valuable in understanding either the context or the action process. The bulk of the cluster communication took place via e-mail. Therefore I requested permission to use these emails as data for my study, in particular those exchanged within the Task Group(s) in charge of developing the information system.

\subsubsection{Data analysis procedures}

Data analysis in an action research study implies identifying and agreeing on criteria in action which can be used to explain what has happened in the system or to indicate that improvement has taken place. Results derived from the data analysis will lead to the development of theories and models that will contribute to the enhancement of knowledge. For Creswell (2003), data analysis involves three basic components: preparing the data for analysis, analysing the data (including gaining in-depth understanding and representing the data), and interpreting the data.

The data need to be organised and processed before any analysis can take place (Miles \& Huberman, 1999; Creswell, 2003). The collected data are usually too "raw": observations are scribbles in a notebook, there are tapes of interviews, documents are piled up, and entries in the journal may have symbols and abbreviations (and in my case, mixed with some Spanish, which is my first language). Transcribing interviews, typing field-notes, and sorting data by date, event, or type of information are steps 
towards preparing data. Miles and Huberman (1999) also indicate that by preparing the data they become an "intelligible product" for anyone to read, edit for accuracy, comment on, code, and analyse.

The second component is the analysis itself. Qualitative methodologies will begin the analysis by coding the refined data (Creswell, 2003). Coding focuses on arranging "chunks" of data (i.e. words, phrases, sentences, and even whole paragraphs) into categories based on particular meanings (Creswell, 2003; Miles \& Huberman, 1999; Jasesick, 1998). This leads to identifying the relationships between codes.

Advanced analysis will engage in the emergence of patterns, trends, and themes (Creswell, 2003). In other words, the analysis moves from what and how to why (Miles \& Haberman, 1999). Jasesick (1998) indicates that, as the analysis progresses, working models explaining the phenomena begin to appear. She also points out that, during data analysis, the researcher is looking for instances to support or challenge the initial theoretical framework of the study.

Creswell (2003), Miles and Huberman (1994), and Morse (1998) indicate that data analysis should be an ongoing process that starts almost at the same time as data collection. Morse (1998) argues that some initial analysis will help in guiding further data collection, and thus avoid the gathering of unnecessary information. On the other hand, Miles and Huberman (1999) mention that the researcher will be able to detect gaps of data and correct this at an early stage. They also argue that if analysis waits until data collection has been completed, not only will it become a daunting job, but it could also discourage the researcher from thinking about new theories to explain the phenomena because they do not have data to support them.

The third component is data interpretation. Here the data are given meaning and there is a focus on expressing what was learned through the research (Creswell, 2003). In the case of the problem-solving cycle in action research, meaning is drawn from the researcher's and participants' interpretation of the problem and how to solve it, which can be fed back to the system as a new intervention (Dickens \& Watkins, 1999). Other complementary outcomes from data analysis mentioned by Creswell (2003) and in line with the research-cycle of action research are: 1) comparing with other results found in the literature or with the initial theoretical framework - if one was declared - thus confirming or disagreeing with them; 2) formulating new and unforeseen questions; and 3 ) interpretation that may call for action, reform, and change. 
As in the case of data collection, action research is ambiguous about which techniques to use for data analysis. One approach proposed by Baskerville and Pries-Heje (1999) to overcome this limitation is to incorporate some grounded theory techniques into action research. Specifically, the authors suggest the integration of grounded theory coding techniques outlined by Glaser and Strauss (1967) into the various phases of action research. Baskerville and Pries-Heje (1999) called this mixed-method "grounded action research" (p.2).

Baskerville and Pries-Heje (1999) first describe the three types of coding that take place in grounded theory approach: open coding, axial coding, and selective coding. Open coding is the first step, where the data are examined for essential ideas which are then grouped into labels or categories. Axial coding follows, with further analysis to identify how data groups are connected to each other. Finally, selective coding will compose the theory that best explains the phenomenon in question based on the previous two coding steps. The appendix in Baskerville and Pries-Heje's (1999) study provides useful information on how to carry out coding and notation.

A search through the literature produced a small number of articles that explicitly indicated how the authors analysed their data. For example, Bell, Giordano, and Putz (2002) mention the use of "grounded theory approach" (p. 15) to analyse interview transcripts, while Whitmore and McKee (2001) collectively developed themes/categories along with their participants. However, both studies failed to elaborate further on the steps taken for data analysis.

One possible reason for this absence of analysis details in articles suggested by Baskerville and Wood-Harper (1998) could be the fact that many action research studies are presented as case research, which has a more relaxed stance towards declaring its research methodology. Orlikowski and Baroudi (1991) also point out that journals' publishing guidelines may limit the authors in providing thorough descriptions about their studies.

$\mathrm{PhD}$ and Masters dissertations employing action research are the best source for learning how other researchers have approached data analysis. Braun (2003a), in her study about the adoption and diffusion of Internet technologies in regional tourism networks, turned to coding according to seven recurring themes that she had established as her initial 7C theoretical framework: connectivity, clustering, communication, collaboration, community, cooperation, and change. Her analysis revealed the role that 
each theme played in the change process of adopting e-commerce, their interdependencies, and which ones were considered more important than others in achieving change.

What I have learned about data analysis from the literature has helped me to reflect on and consider the approach to follow. I prepared the raw data for its initial open coding analysis by writing up my observational notes. Interviews were transcribed shortly after they had been held and sent to the interviewee for verification and correction. Printed data such as reports and letters collected during the fieldwork were stored in a filing cabinet labelled by type and date. Electronic data, such as e-mails and other files, were stored in a directory I created in my computer.

I followed the advice of Creswell (2003), Miles and Huberman (1999), and Morse (1998) on employing a computer programme designed to assist in content management and data analysis. I opted for Nvivo 2.0. This programme has been useful in my analysis as it allows cross-referencing of entries, locating quotations and remarks, linking with the original sources, and recording the multiple perspectives that one category or theme can have.

I converted the collected observational notes and interviews into Rich Text Format (RTF) files in order to import them into Nvivo. I was able to obtain the electronic versions for most of the clusters' agendas and minutes, which I also converted into RTF files. Other electronic data such as e-mails were also converted to RTF format.

Being a qualitative study, my analysis process is based on reduction and interpretation (Creswell, 1994). I took the collected data and reduced them to certain patterns, categories, or themes. I paid special attention to the grounded theory techniques for data analysis. With the assistance of Nvivo I began my open coding by grouping chunks of text from the data collected into categories (Nvivo calls these categories "nodes"). In my axial coding I started to link categories with one another and drew diagrams illustrating their relationships. Finally with selective coding I mapped the bidding process and proposed where ICT could support knowledge transfer; as well as interpreted how the cluster's digital knowledge transfer mechanisms were developed, how the cluster's knowledge-base in project bidding is developed, and what are the factors that influence the growth of this knowledge-base. 
The specific processes used to reduce the different source of data for the stages of this action research intervention are discussed in Part 2 - Chapters 5, 6, 7, 8, and 9. The interpretation of findings is discussed in Part 3 - Chapters 10, 11, 12, and 13.

\subsubsection{Exiting the site}

McNiff (1988) was of the view that action research has a self-generative capacity; in other words, the main spiral of planning, acting, observing, reflecting, and re-planning may develop spin-off spirals to explore other problems potentially arising during the inquiry. Similarly, West and Stansfield (2001) commented that the cycles in action research can become "never-ending" learning opportunities.

Although learning is one of the reasons for carrying out action research, it is not realistic that the researcher stays on site indefinitely. Checkland and Holwell (2007) are of the view that it is up to the researcher's judgement when enough learning has taken place from following a methodology and applying a theoretical framework to the realworld situation. At this point they should conclude the action research study.

For Jönsson (1991), one aspect of exiting the site is the negotiation of a pre-set termination date with the host organisation. A termination date makes it easier for the researcher to disengage from the ongoing processes that take place in the organisation and to focus on performing the interpretive work of data analysis (Checkland, 1991; Jönsson, 1991).

On the other hand, defining a tentative termination date is also relevant for the host organisation, as West's and Stansfield's (2001) experiences corroborate. Participants in their respective studies wanted to know at the beginning when the studies were expected to conclude in order to assess the time and resources they needed to commit.

Jönsson (1991) also considered that reporting back to the host organisation should be part of exiting the site. For him, the host organisation should be the first one to hear the findings of the study - especially if these were controversial.

For the case of this study, exiting the clusters was determined by the completion of a specific project rather than agreeing to a pre-set termination date. I worked with the clusters for a period of three years (from mid-2003 to mid-2006). As previously stated, in my first year I worked as a volunteer for the clusters while writing up my research proposal. During the following two years, the clusters and I engaged in the practical 
problem-solving cycle of the action research enquiry, which culminated with the development of an online portal for Natural Hazards New Zealand. I exited the site shortly after the portal went live to allow myself time to complete the research-cycle component of the $\mathrm{PhD}$ programme.

As part of my exit from the site, I prepared a written report for the clusters and other stakeholders about the findings and conclusions of the study. I also organised an oral presentation for both clusters.

\subsubsection{The narrative of the study}

My study targets two different audiences: cluster members (practitioners) and the IS community (academics). Therefore, I produced two written reports that follow different types of narrative.

For the case of cluster members and other stakeholders, I followed a similar format to that used by Harvey and Gray (2003) for reporting cluster profiles to Positively Wellington Business. Their report was a concise account of the background to the study, methodology, findings, analysis, and assessment. I paid attention to the practical lessons and findings that emerged during the study rather than to those of a theoretical character.

Regarding the written report for the IS community, I followed the style adopted by Braun (2003a), Yoong (1996), and West and Stansfield (2001). Their studies provided a detailed description of the concepts and context of the systems they studied. They then detailed the action during each of the stages in their action cycles, and finally presented their thoughts and comments on what was learned from the action.

Like these authors, I first introduced the system and its context. Then I carried out one cycle of action and learning.

I was also interested in including the participants' opinions and points of view in the form of embedded quotes. I want my narrative to be rich and descriptive to help the audience understand the situation and the reasons behind the conclusions I reached. 


\section{PART 2:}

\section{THE ACTION RESEARCH INTERVENTION}

Cluster members and I worked together to improve how the clusters managed their knowledge-base to ensure it was readily available for sharing or for future reference. Part 2 firstly provides details of the clusters' context and then discusses how teams tackled the practical problem of developing an ICT solution. The intervention followed the action research stages of diagnosis, planning, development, and evaluation. These stages give their names to the Chapters in Part 2. 


\section{Chapter 5: The context of the study}

The clusters of my study are embedded in a historical, political, and socio-economic dimension that continuously shapes their development and performance. To understand the actions taken during the intervention and their outcome, it is important to acknowledge this external context and how Earthquake Engineering and Natural Hazards were conceived.

This Chapter is a summary produced mainly by analysing the documents gathered during my time with the clusters. In this instance, information was coded following setting and context codes (Creswell, 1994) at three levels: national, regional and cluster. The documents collected and analysed ranged from official government and local authority reports and newsletter publications to internal cluster documentation.

\subsection{The national economic and political context}

New Zealand enjoyed an enviable third position in the world regarding standards of living per capita during the 1950s (ITAG, 1999; MED, 2002); however, by 2007, our country ranked 22nd among the thirty OECD countries (MED, 2007). Although New Zealand's real per capita income continued to grow during those fifty years, other countries managed to outpace our nation. Countries such as Ireland and Singapore embraced the premises of the Knowledge Economy and were successful in making the transition (ITAG, 1999).

In 1991 Professor Michael Porter of Harvard Business School and his colleagues were invited to New Zealand by the Government to assess the country's competitive position and its economic options in the Knowledge Economy. Their project was called Upgrading New Zealand's Competitive Advantage but was more commonly known as the "Porter Project" (Crocombe et al., 1991; Brimblecombe, 1999). The key activity of the project involved using Porter's model of competitive advantage to analyse the sources of advantage of 20 New Zealand export industries.

The findings revealed that New Zealand relied on the exploitation of favourable temperate-climate conditions which allowed the country to produce commodities at low cost, rather than on the creation of more sophisticated advantages. Consequently, New Zealand is an exporter in structurally unattractive industries and segments. The Porter Project made clear the urgent need for a new economic order better matching New 
Zealand's social aspirations but also aligned with the mandates of modern economic competition (Crocombe et al., 1991).

The New Zealand Government studied international trends and assessed the country's position in the international economic arena (ITAG, 1999). Attention was drawn to the fact that the greatest economic benefits in countries such as Ireland and Finland have been generated from innovative and knowledge-based activities rather than from traditional commodity-based ones. From these findings, the New Zealand Government identified innovation as a key component in its strategy for economic growth (The Office of the Prime Minister, 2002; MED, 2002).

The Government has set the economic goal of getting New Zealand back to the top half of the OECD's per capita income rankings - and remaining there (MED, 2002; MED \& The Treasury, 2005). Given the fact that only businesses can generate wealth or economic growth, the New Zealand government will work towards such a goal by enabling business establishment and growth through regulation and direction, active support, and facilitation activities (MED, 2002; The Design Taskforce, 2003; The Office of the Prime Minister, 2002).

Industry New Zealand, the Government's economic and development agency, was given the task of coordinating with local governments and the private sector to promote regional economic growth, by developing specific programmes focused on helping world-class businesses to commercialise their innovative products and services, assisting them in the internationalisation process, and developing collaborative networks (MED, 2002; Industry New Zealand, 2002).

In this context, Industry New Zealand acknowledged the role of clusters for regional economic growth and conceptualised a Programme in collaboration with industry and regional economic development agencies (Industry New Zealand, 2001). The Clusters Development Programme (CDP) was launched in 2003 by New Zealand Trade and Enterprise (NZTE, after the merger of Industry New Zealand with Trade New Zealand).

The CDP aimed at building specialised, knowledge-rich, and internationally competitive clusters by bringing together key companies, funding a cluster Facilitator for a two-year period, and providing the clusters with tools and support materials (MED, 2003 \& 2005). The Programme provided support to eighty-two clusters, amongst them the two clusters of this study: Earthquake Engineering New Zealand (EENZ) and Natural Hazards New Zealand (NHNZ). 
During the Cluster Facilitator Workshop organised by NZTE in June 2004, a change of focus was announced with regard to the Cluster Programme. The Programme would now be part of a sectorial approach rather than standing by itself, and therefore clusters were allocated to one of the following sectors: ICT, tourism, manufacturing, food and beverages, education, creative, and wood processing:

Unfortunately, EENZ and NHNZ did not really fit any of the sectors or groups that NZTE has created. This was reflected during the workshop, as both clusters were placed with the Auckland-based Logistics cluster in an additional category created on the spot as "Services."

The CDP was disestablished in 2006 after a change in policy. It was decided that the Programme did not have clear objectives and quantifiable economic outcomes. Additional factors leading to the decision included the variability of the programme recipients and the existence of other NZTE programmes that duplicated some of the Programme's activities (MED, 2005). Government support to clusters continues, but in an indirect manner through other NZTE programmes and funding mechanisms that do not exclude collaborative groups such as the EGD-MD Programme or the Regional Partnership Programme.

\subsection{The regional context}

The Wellington region is located at the bottom of New Zealand's North Island (see Figure 11). In the 2006 National Census the region had a population of 448,956. It comprises eight councils: Carterton District, Kapiti Coast District, Lower Hutt City, Masterton District, Porirua City, Upper Hutt City, Wellington City, and South Wairarapa District. Wellington is also the name of the region's main city, which is New Zealand's capital and thus the country's political centre. 


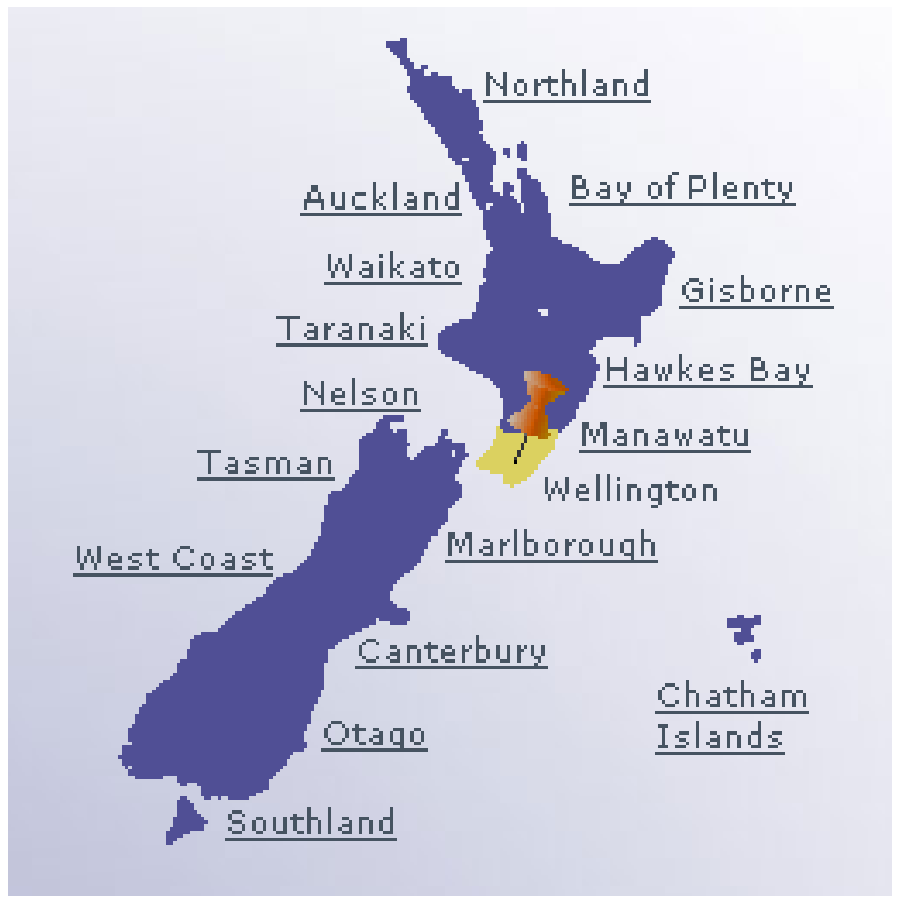

Figure 11. The Wellington region (source: www.localcouncils.govt.nz)

Brimblecombe (1999) gives an overview of the genesis of cluster development in the Wellington region. She first describes how the region's economic make-up faced major changes in the 1980s and 1990s due to the closing-down, down-sizing, or relocation of the then-predominant textile, automotive, food processing, and heavy engineering industries. Nevertheless the region - and in particular Wellington City - was still considered service-driven, as it hosts New Zealand's central government and major corporate financial entities.

In 1997 Wellington City and the region's other three cities (Lower Hutt, Upper Hutt, and Porirua) conceived the Wellington Business Cluster Project. The Project aimed to develop a new economic base for the region with an export-oriented focus (Brimblecombe, 1999; Harvey \& Gray, 2003). The Project was managed by the Wellington Regional Economic Development Agency (WREDA), an agency created in partnership between these cities.

The cluster approach was identified as a critical tool for the future growth of the region's economy. The project organised over 300 local businesses, government, tertiary education, and scientific participants to discuss collaborative initiatives to tackle issues that were commonly affecting them, such as: identifying infrastructural, skills and policy gaps; exploring new markets; and developing joint marketing programmes (WREDA, 2002a). 
The Wellington Business Cluster Project began to take shape with initial surveys and sector group meetings. With the support of NZTE and external consultants the Project triggered networking activity and identified cluster development opportunities based on existing agglomerations of industries in the region (Brimblecombe, 1999).

The Project identified three potential clusters that could be developed further through some short-term catalyst funding: Film and Television, Information Technology Telecommunications and Electronics, and Earthquake Engineering Technology. The Content Creation group was added later as a fourth cluster.

Brimblecombe (1999) concluded that, although there was a crucial "top-down" facilitative role played by the regional development agencies who executed the first stages of the Wellington Business Cluster Project, there was also an effective "bottomup" involvement by cluster members who took ownership of this initiative and further developed it.

By 2002 the Wellington Business Cluster Programme peaked with 11 clusters in the fields of earthquake engineering, natural hazards, software, e-business, creative content, film and television, education, mobile Internet, optics, Maori, and Pacific Island consultants (WREDA, 2002b). These clusters were in various stages of development, from initiation to improvement and renewal.

However at the same time the 2002/2003 Annual Cluster Profiles report prepared by Harvey and Gray (2003) for Positively Wellington Business (PWB) - the new name for the Wellington region's economic development agency - pointed out some key challenges faced by the clusters in the Programme.

For instance, the report indicated that three of the leading clusters in the Programme (Earthquake Engineering, Creative Capital, and Natural Hazards) have reached a stage in their development where members are comfortable working together and have a brand and common objectives. However, additional funding is required to sustain marketing support to continue the clusters' momentum in export markets, developing relationships, gathering market intelligence, identifying prospects, and converting these into business opportunities.

In 2003 two cases were put forward, one to Industry New Zealand (now part of NZTE) and the other to Wellington City Council, requesting increased support for these clusters, both of which were rejected. It seems that the cluster concept catches interest at national and regional level to fund start-ups, but after that funding is reduced: 
[Cluster Facilitator] noted that the base funding for the cluster is about $50 \%$ less than 2 years ago. He noted that Positively Wellington Business is currently negotiating with NZTE for a package of funding support with the aim of providing additional marketing assistance for the clusters. At this stage the outcome as far as EENZ is concerned is uncertain. (EENZ Minutes, 04 March 2004).

Another challenge mentioned in Harvey and Gray's (2003) report was PWB's adoption of a sectoral approach to regional economic development, as a response to Central Government change of policy in this field. By this means the clusters became a subset. According to these authors, PWB needed to clarify to stakeholders where it would place its emphasis in terms of strategy and resources.

\subsection{Earthquake Engineering NZ and Natural Hazards NZ clusters}

New Zealand has been acknowledged internationally as a leader in the development of earthquake mitigating structures, retrofitting techniques, base isolation, natural disaster risk assessment, and insurance systems. One key example is Dr Bill Robinson's leadrubber bearing base isolators for buildings, which were put to the test during the 1994 Los Angeles and 1995 Kobe earthquakes.

Wellington City has had a long association with the earthquake engineering industry due to its high earthquake risk. Several iconic buildings in Wellington have been built or retrofitted using base isolation technology, amongst them Te Papa (The National Museum of New Zealand), Parliament House, and Victoria University's Hunter Building. As the capital city of New Zealand, Wellington congregates Government Ministries and key agencies, such as the Ministry of Civil Defence and Emergency Management, the Earthquake Commission, research institutes, and universities, as well as head offices and/or regional offices of leading private sector companies involved in professional engineering, risk insurance, and relevant disciplines.

Earthquake Engineering New Zealand (EENZ) and Natural Hazards New Zealand (NHNZ) are two clusters of professional services that were conceived in this context. These two clusters have been sponsored by PWB, which assists with some targeted small marketing grants and administration time by one of the PWB officials ${ }^{12}$. In

\footnotetext{
12 PWB also used to fund the part-time facilitator. The costs of the facilitator are now covered by members' fees but his time has been drastically reduced. The facilitator plays a key role for the clusters as he provides business development and marketing support.
} 
addition, the Government has been a supportive partner for specific cluster projects through NZTE, Ministry of Foreign Affairs, and NZAID (EENZ \& NHNZ, 2007).

Given that their inception is closely tightened with regional economic development initiatives and their effort to embody a range of related industries within the region, EENZ and NHNZ can be considered regional clusters.

EENZ is comprised of engineers and scientists in earthquake protection engineering for infrastructure such as buildings, bridges, and roads. The key drivers for the formation of the cluster included: developing the international profile for New Zealand earthquake engineering services and products; growing international revenue for New Zealand business; and providing career prospects to attract and retain professionals in the sector (Carroll, 2006).

This cluster was established in 1998 as a Capital Development Agency (the predecessor of Wellington Regional Development Agency, which later became Positively Wellington Business) initiative to foster knowledge sharing, develop ideas for new business opportunities, and facilitate collaboration to tackle international projects (Brimblecombe, 1999). EENZ currently has over thirty company members, some of which are large players in the international arena. Others are small companies, research institutes, and university departments.

NHNZ was formed in 2000 as a spin-off from EENZ. It is a group with expertise in risk management, recovery, and mitigation from natural hazards, including earthquakes, tsunamis, cyclones, climate change, floods, landslides, volcanic eruption, fires, coastal erosion, and drought. Like EENZ, this cluster is comprised of around thirty members drawn from companies involved in research, emergency management, consultancy, engineering, and education. The size of its members varies from one-man firms to large international companies.

Due to the overlapping nature of their areas of expertise, some members of EENZ are also part of NHNZ. The clusters have collaborated in numerous international bid opportunities and project initiatives in Turkey, Romania, UAE, Iran, the Pacific, and Asia (in particular Indonesia and Vietnam):

Some $\$ 33$ million of projects have been identified and bid on by collaborative initiatives since establishing the Earthquake Engineering NZ and associated Natural Hazards NZ business clusters. \$2.25 million worth of projects have been won with identified prospects and opportunities worth over $\$ 17$ million. (EENZ Minutes, 28 April 2004). 
EENZ and NHNZ joined forces in early 2003 to apply for funding from the Government's Clusters Development Programme (CDP) and were successful in receiving assistance for two years to cover the costs of a facilitator. The clusters have also worked together in marketing initiatives that raised their profile with the Asian Development Bank (ADB), the World Bank, and in specific countries such as Turkey, India, and China. For example, they assisted Wellington City Council in hosting the Earthquake Disaster Mitigation delegation from their sister city Tianjin in 2004.

During the process of international project bidding and marketing, knowledge has been shared and created by the clusters:

We now know a lot more about this new natural hazards international market than when we started 3 years ago. This has positioned the cluster to win significant project work in the next 3 years. In summary, the Cluster is well worth having. (NHNZ Minutes, 11 March 2004).

The extent and sustained nature of the bidding and marketing processes led to the development of common templates, including standardised CVs, experts, and company profiles and track record material. It has also led to an understanding of the specialist skills, capabilities, and resources available within the clusters, and to the building of important relationships and trust (Carroll, 2006).

The level of maturity by both clusters was also acknowledged in the report Collaborative Cluster and Networks Profile 2004 produced by Gittos, van Opdorp, and Gray (2004), where EENZ and NHNZ were considered to be in the Improvement Stage of Gray et al's (2001) cluster development model depicted in Figure 3. EENZ and NHNZ have surpassed their initial development stages and have collaborated internationally in numerous project bidding and marketing opportunities. This has increased the level of information sharing and assistance between cluster members.

Currently both clusters operate with limited resources. This poses a challenge as they are at a stage requiring sustained momentum in export markets, developing relationships, gathering market intelligence, and identifying prospects to convert into business opportunities.

As mature clusters, EENZ and NHNZ possess a good knowledge-base. Both clusters have web presence and some systems and procedures in place. The websites however have focused on the marketing side of things and clusters have not used them as a tool for knowledge transfer in their bidding activities. 


\section{Chapter 6: The Diagnosing Stage}

The Diagnosing Stage was a self-contained piece of research that answered my first research question:

\section{What are the knowledge transfer mechanisms in a cluster when it is performing a business activity such as international project bidding?}

This posed the problem of how to report the findings of this stage in my dissertation. I first tried to report these as part of the action cycle. Although this approach provided a logical flow of the events that took place and the thinking behind these, the findings from the Diagnosis Stage became overshadowed by the subsequent stages of planning, action taking, and evaluating. Furthermore, these findings became separated from the rest of the study's discussion of findings at the specifying learning stage.

After some consideration and discussion with my supervisor, I opted to submit the findings of this stage with my other findings. Therefore in this Chapter I provide a summary so that the reader is not faced with a gap in the occurrences and achievements at the Diagnosis Stage. This may cause the reader to feel that some of the statements in this Chapter do not have supporting evidence. I request the reader to refer to the detailed analysis of the findings for this stage in Chapter 10.

\subsection{Executing the Diagnosis Stage}

The diagnosing stage was the starting point of this action research study. The purpose of diagnosing was to describe the knowledge transfer mechanisms currently employed by NHNZ and EENZ for their international project bidding process. Based on the findings of this stage, the researcher and a team from the NHNZ cluster moved on to planning the intervention.

For the Diagnosis Stage, I took observational notes at the cluster bi-monthly meetings and other meetings I was invited to attend, such as the preparation of the proposal for a project in the Gujarat Province, India. I made regular entries in my journal to record my personal thoughts and reflections about the project bidding process. I interviewed the cluster Facilitator and three cluster members who have recently participated in project bids. Finally, I requested permission to use e-mails regarding project bidding that have been circulated around the clusters. This has allowed me to record the evolution of several projects for which NHNZ and EENZ were bidding. 
I analysed the data collected by coding chunks of text into categories which tend to resemble steps of a process. Refinement of my coding led to a clear definition of the project bidding process. This allowed me to apply genre systems analysis to each of the steps in the process and map the genres being called upon by the clusters.

I maintained a more dominant position in this first stage, as I designed the research process, collected the data, and interpreted it. The participants contributed with feedback on interim results, and modified some of the data interpretation to align it with the actual situation. They also validated the final report for this stage.

\subsection{Project bidding in EENZ and NHNZ}

Bidding for international projects in the natural hazards and earthquake engineering arenas is not only highly competitive, it is also a long, sophisticated, and costly process. Members - regardless of their size - acknowledge that working in a collaborative cluster approach has enabled them to participate in a greater range and number of project bids than would have been possible had they tendered individually:

The assistance of the cluster collaborative approach to help achieve the winning of this World Bank tender from tough international competition appreciated. (Minutes NHNZ cluster meeting, 26 May 2004).

The cluster approach ensures that suitable specialist teams are put forward for particular bids, thus increasing the chances of winning an overseas tender.

When bidding for a specific project, EENZ and NHNZ clusters follow the approach of appointing one of their company members as the lead consultant. The lead company will then assemble a team of top specialists from amongst the cluster members to fit the project's requirements.

In general terms, the bidding process involves identifying the opportunity, alerting the cluster about it, setting up a group of companies who are interested in pursuing the opportunity, and submitting a proposal. If the group is successful, it negotiates the final contract with the client and then starts the project. Beginning work on the project is not considered to be the end of the bidding process but the beginning of a new bidding cycle, as those working on the ground have the chance to build relationships and be alerted to new opportunities that their companies and others in the cluster may be interested in pursuing. 
The cluster as a whole is involved at the beginning stages of the project bidding process, but later it takes a less active role. As one interviewee indicated, the cluster is there "to get you there." The responsibility of preparing a bid falls upon a smaller team and the lead company.

\subsection{The proactive and reactive approaches in project bidding}

The Diagnosis Stage identified two types of approach that the clusters tend to follow during project bidding: reactive and proactive. The reactive approach takes place when the cluster responds to a procurement notice requesting consultants to put forward proposals. In the proactive approach, on the other hand, the cluster has actively been involved in creating the opportunity. It is important to point out that not all bids fit nicely into one of these two approaches; there could be situations where a mix of both approaches occurs. A diagram and detailed description for the reactive and proactive approaches are found in sections 10.1 and 10.2 respectively.

\subsection{Identifying the genres in project bidding}

The collaborative interactions (i.e. genres) of the clusters in both reactive and proactive approaches were mapped through a genre systems lens. "Project Bidding" was the name of a coordinated and distinctive communicative system whose primary purpose from the clusters' perspective - is to be selected as the suitable contractor to carry out a specific project.

The Project Bidding system was broken down into a series of steps or sub-systems, such as: alert cluster, informal networking, research opportunity further, etc. Each sub-system involves a sequence of genres that communicate something in order to achieve an expected action. Appendix E provides the complete list of the genres found in the Project Bidding system with details from Genre Systems analysis.

For example, the step Prepare EOI (R.10 of the reactive project bidding approach) in Figure 17 involves assembling an Expression of Interest (EOI) to introduce the team, their capabilities, and their track record on similar jobs, according to determined specifications by the client's EOI notice. In order to complete this task, the following genres were used (see Table 6): Procurement Notice, Request for Expression of Interest, EOI meeting, Request Call, Reply Message, Expert Profile, Project Sheet, and Company brochure. 


\begin{tabular}{|c|c|c|c|c|c|c|}
\hline Genre & Purpose & Content & Actors (i) & Form & Time & Place \\
\hline $\begin{array}{l}\text { Procurement Notices } \\
\text { (World Bank) }\end{array}$ & $\begin{array}{l}\text { Invite eligible consultants } \\
\text { to register their interest in } \\
\text { carrying out the project. } \\
\text { List what the EOl should } \\
\text { include. }\end{array}$ & $\begin{array}{l}\text { Title: Procurement Notice. } \\
\text { Then there is a set of key } \\
\text { information listed in a } \\
\text { table: country, project, } \\
\text { financing, abstract, } \\
\text { sector, loanicredit } \\
\text { number, contractibid } \\
\text { number, and deadline. } \\
\text { After that, there is a one- } \\
\text { page summary of: context } \\
\text { of the project, objectives, } \\
\text { key activities and eligibility } \\
\text { of bidders. Finally, } \\
\text { specifications of what the } \\
\text { EOl should include and } \\
\text { the contact details where } \\
\text { the EOI should be } \\
\text { subnitted and its } \\
\text { deadline. }\end{array}$ & $\begin{array}{l}\text { World Bank, the funding } \\
\text { recipient (client), NZTE } \\
\text { Washington Office, PME } \\
\text { Liaison, cluster members. }\end{array}$ & $\begin{array}{l}\text { Medium: Email } \\
\text { (distribution list). } \\
\text { Structure: Formal } \\
\text { contractual there is a } \\
\text { table with the key details } \\
\text { such as name of project, } \\
\text { country and project ID. } \\
\text { The procurement notice is } \\
\text { divided into pre- } \\
\text { determined headings. } \\
\text { Linguistics: Formal } \\
\text { contract. }\end{array}$ & $\begin{array}{l}\text { Up to } 3 \text { weeks, but } \\
\text { usually less than that. } \\
\text { View at any time within } \\
\text { that frame. }\end{array}$ & $\begin{array}{l}\text { World Bank Website, } \\
\text { Outlook. Any place. }\end{array}$ \\
\hline $\begin{array}{c}\text { Request for Expressions } \\
\text { of Interest }\end{array}$ & $\begin{array}{l}\text { Invite eligible consultants } \\
\text { to register their interest in } \\
\text { carrying out the project. } \\
\text { List what the EOI should } \\
\text { include. }\end{array}$ & $\begin{array}{l}\text { Title of the project. } \\
\text { Summary of: context of } \\
\text { the project, objectives, } \\
\text { key activities and details } \\
\text { of bidders' eligibility. } \\
\text { Finally, specifications of } \\
\text { what the EOI should } \\
\text { include and the contact } \\
\text { details where the EOI } \\
\text { should be submilted and } \\
\text { its deadline. }\end{array}$ & \begin{tabular}{|l|} 
The client, NZTE \\
commissioner, PMV \\
Liaison, cluster members.
\end{tabular} & $\begin{array}{l}\text { Medium: Email } \\
\text { (distribution list) with } \\
\text { attachnent. } \\
\text { Structure: Formal } \\
\text { contractual The request } \\
\text { is divided in pre- } \\
\text { determined headings. } \\
\text { Linguistics: Formal } \\
\text { contract. }\end{array}$ & $\begin{array}{l}\text { Up to } 3 \text { weeks, but } \\
\text { usually less than that. } \\
\text { View at any time within } \\
\text { that frame. }\end{array}$ & Outlook. Any place. \\
\hline Prepare EOI Meeting & $\begin{array}{l}\text { Coordinate the assembly } \\
\text { of the EOI. }\end{array}$ & $\begin{array}{l}\text { Discussion about what to } \\
\text { include in the EOI, } \\
\text { regarding methodology, } \\
\text { CVs and requests for } \\
\text { material that needs to be } \\
\text { part of the EOI. }\end{array}$ & $\begin{array}{l}\text { Team leader, EOI } \\
\text { Coordinator, rest of EOI } \\
\text { team, cluster Facilitator. }\end{array}$ & $\begin{array}{l}\text { Medium: Face to face } \\
\text { meeting, telephone } \\
\text { conference. } \\
\text { Structure: } \\
\text { tollowsuctured, } \\
\text { the team leadenda set by } \\
\text { Co-ordinator. } \\
\text { Linguistics: Informal, } \\
\text { conversation. } \\
\end{array}$ & $\begin{array}{l}\text { Urgent, within the } \\
\text { submission's deadline. } \\
\text { Same time within that } \\
\text { frame. }\end{array}$ & $\begin{array}{l}\text { At one of the interested } \\
\text { parties' premises. Same } \\
\text { place. If telephone- } \\
\text { conferencing is being } \\
\text { used, then different } \\
\text { places. }\end{array}$ \\
\hline Request Call & $\begin{array}{l}\text { Request tean members } \\
\text { for further information to } \\
\text { complete the EOI } \\
\text { document. }\end{array}$ & $\begin{array}{l}\text { Greetings, request } \\
\text { specific information } \\
\text { farewell. }\end{array}$ & $\begin{array}{l}\text { Cluster member who is } \\
\text { part of the bidding team, } \\
\text { team leader, cluster } \\
\text { Faciltator. }\end{array}$ & $\begin{array}{l}\text { Medium: Telephone } \\
\text { conference. } \\
\text { Structure: Formal } \\
\text { business meeting. } \\
\text { Linguistics: Formal, } \\
\text { business-oriented. }\end{array}$ & Same time. & $\begin{array}{l}\text { Telephone line. Different } \\
\text { place. }\end{array}$ \\
\hline Reply Message & $\begin{array}{l}\text { Reply to request. Provide } \\
\text { the additional information } \\
\text { needed to complete the } \\
\text { EOl document. }\end{array}$ & $\begin{array}{l}\text { Greetings, submission of } \\
\text { specific information, } \\
\text { farewell. }\end{array}$ & $\begin{array}{l}\text { EOl team member, } \\
\text { team leader. }\end{array}$ & $\begin{array}{l}\text { Medium: Email, usually } \\
\text { with key information } \\
\text { attached. } \\
\text { Structure: Greetings, } \\
\text { reasons for writing the } \\
\text { email and farewell. } \\
\text { Language: Informal but } \\
\text { professional. }\end{array}$ & $\begin{array}{l}\text { Urgent, within the } \\
\text { submission's deadline. } \\
\text { Any time within that } \\
\text { frame. }\end{array}$ & Outlook. Any place. \\
\hline Expert Profile & $\begin{array}{l}\text { Provide information about } \\
\text { the expert. }\end{array}$ & $\begin{array}{l}\text { Information about the } \\
\text { expert's contact details, } \\
\text { work history, record of } \\
\text { work in similar projects, } \\
\text { education and training. }\end{array}$ & $\begin{array}{l}\text { Tean leader, EOI team, } \\
\text { cluster Facilitator. }\end{array}$ & $\begin{array}{l}\text { Medium: Document; it is } \\
\text { usually distributed via } \\
\text { email. Structure: Very } \\
\text { structured, it follows pre- } \\
\text { deternined headings. } \\
\text { Linguistics: Formal, } \\
\text { business-oriented. }\end{array}$ & $\begin{array}{l}\text { Urgent, within the } \\
\text { submission's deadline. } \\
\text { Any time within that } \\
\text { frame. }\end{array}$ & Any place. \\
\hline $\begin{array}{l}\text { Project Sheet } \\
\text { (Track Record) }\end{array}$ & $\begin{array}{l}\text { Provide track record of } \\
\text { previous jobs done by } \\
\text { cluster members who are } \\
\text { part of the EOI team. }\end{array}$ & $\begin{array}{l}\text { Information about work in } \\
\text { previous projects with } \\
\text { similar characteristics. } \\
\text { Some details are: project } \\
\text { name, client, value of } \\
\text { project, description of } \\
\text { project, specific tasks } \\
\text { performed in the project. }\end{array}$ & $\begin{array}{l}\text { Team member, team } \\
\text { leader. }\end{array}$ & $\begin{array}{l}\text { Medium: Document; it is } \\
\text { usually distributed via } \\
\text { ernail. } \\
\text { Structure: Very } \\
\text { structured, it follows a } \\
\text { template. } \\
\text { Linguistics: Formal, } \\
\text { highly technical business- } \\
\text { oriented. } \\
\end{array}$ & $\begin{array}{l}\text { Within the EOl's deadline. } \\
\text { Any time within that } \\
\text { trame. }\end{array}$ & Same place. \\
\hline $\begin{array}{l}\text { Expression of Interest } \\
\text { (EOI) }\end{array}$ & $\begin{array}{l}\text { To be considered for the } \\
\text { tender short-list. } \\
\text { Introduce the team of } \\
\text { experts and showcase } \\
\text { their work experience on } \\
\text { the field. }\end{array}$ & $\begin{array}{l}\text { Cover page with pictures, } \\
\text { introduction (why use this } \\
\text { group of consultants, our } \\
\text { understanding of the } \\
\text { project), areas of } \\
\text { expertise, companies' } \\
\text { description and } \\
\text { capabilities, records of } \\
\text { previous jobs, list of } \\
\text { experts and their CVs, } \\
\text { contact details, } \\
\text { conclusion. }\end{array}$ & $\begin{array}{l}\text { EOI team, local partner, } \\
\text { client. }\end{array}$ & $\begin{array}{l}\text { Medium: Printed } \\
\text { document, fax, email. } \\
\text { Structure: Very } \\
\text { structured, it follows a } \\
\text { template. Linguistics: } \\
\text { Formal, highly technical } \\
\text { and business-oriented. }\end{array}$ & $\begin{array}{l}\text { Urgent, within the } \\
\text { submission's deadline. } \\
\text { Any time within that } \\
\text { frame. }\end{array}$ & Any place. \\
\hline Company Erochure & $\begin{array}{l}\text { To showcase the } \\
\text { companies who are part } \\
\text { of the team. }\end{array}$ & $\begin{array}{l}\text { Set of glossy material and } \\
\text { brochures describing the } \\
\text { companies and their } \\
\text { capabilities that is } \\
\text { attached to the EOI. }\end{array}$ & $\begin{array}{l}\text { EOI team, local partners, } \\
\text { client. }\end{array}$ & \begin{tabular}{|l|} 
Medium: Printed \\
document posted by \\
courier \\
Structure: Very \\
structured, marketing- \\
type format. \\
Linguistics: Formal, \\
technical and \\
businessinarketing. \\
\end{tabular} & Any time. & Any place. \\
\hline
\end{tabular}

(i) The highlighted actors are the ones who usually initiate the genre.

Table 6. The genres found in the sub-system "Prepare EOI." 
The Genre Systems framework looked at each collaborative interaction in terms of purpose, content, actors, form, time, and place. In the case of the sub-system "Prepare EOI," there was a variety of genres, many of which were be invoked several times. There is a sense of urgency due to the short time before the deadline. Most of the actors were within the team that was preparing the EOI. There was a high volume of traffic for requests for information, track records, and $\mathrm{CVs}$ by the team leader, the EOI coordinator, and other team members. Usually these genres were delivered via e-mail.

Genre systems analysis also showed that certain sub-systems not only contained a large variety of genres, but also that their traffic level was high. These findings are discussed in detailed in Chapter 10, as such sub-systems were candidates for ICT support initiatives. 


\section{Chapter 7: The Planning Stage}

Around the time that the Diagnosis Stage report was taking shape, Natural Hazards New Zealand (NHNZ) prepared to undertake the task of updating their old website. This enabled the opportunity to consider the preliminary findings of the Diagnosis Stage in the website review and deliver a solution for NHNZ that not only addressed their marketing and product promoting needs, but also supported the project bidding process.

A team comprised of cluster members and I worked together towards designing the new NHNZ website. The main objectives of this stage were to conceptualise the new website and to prepare a Website Specifications Document.

Given the fact that planning for the new NHNZ website was a process taking place between 11 March 2004 and 26 May 2004, my analysis focused on examining key cluster events associated with the project. I followed Miles and Huberman's (1994) advice and created a matrix to systematically display the data in chronological order. By so doing I was able to understand the flow and connection of events and this allowed me to write the "story" of the Diagnosis Stage.

I also continued with open and axial coding of the data collected throughout this stage to develop categories that later fed into the interpretation of findings detailed in Chapters 12 and 13.

To appreciate the relevance of the decision to update the NHNZ old website, it is important to understand the context in which it took place. The following sub-section will provide background information on the need to review the website.

\subsection{The "old" NHNZ website ${ }^{13}$}

NHNZ had a small website, built shortly after the inception of the NHNZ cluster in November 2000. This website remained unchanged except for minor maintenance in content. The "old" website was hosted by a cluster member at a minimal cost.

The old website's design and structure was simple. The Home Page provided general information about what NHNZ do. From there the visitor could go to pages that described NHNZ, detailed the types of services they provide, listed cluster members,

\footnotetext{
${ }^{13}$ For the purposes of this report, this website will be called the "old" website to differentiate it from the one the cluster was planning to develop.
} 
listed projects members have undertaken in the past, and provided interesting links, news, and contact details (see Figure 12).

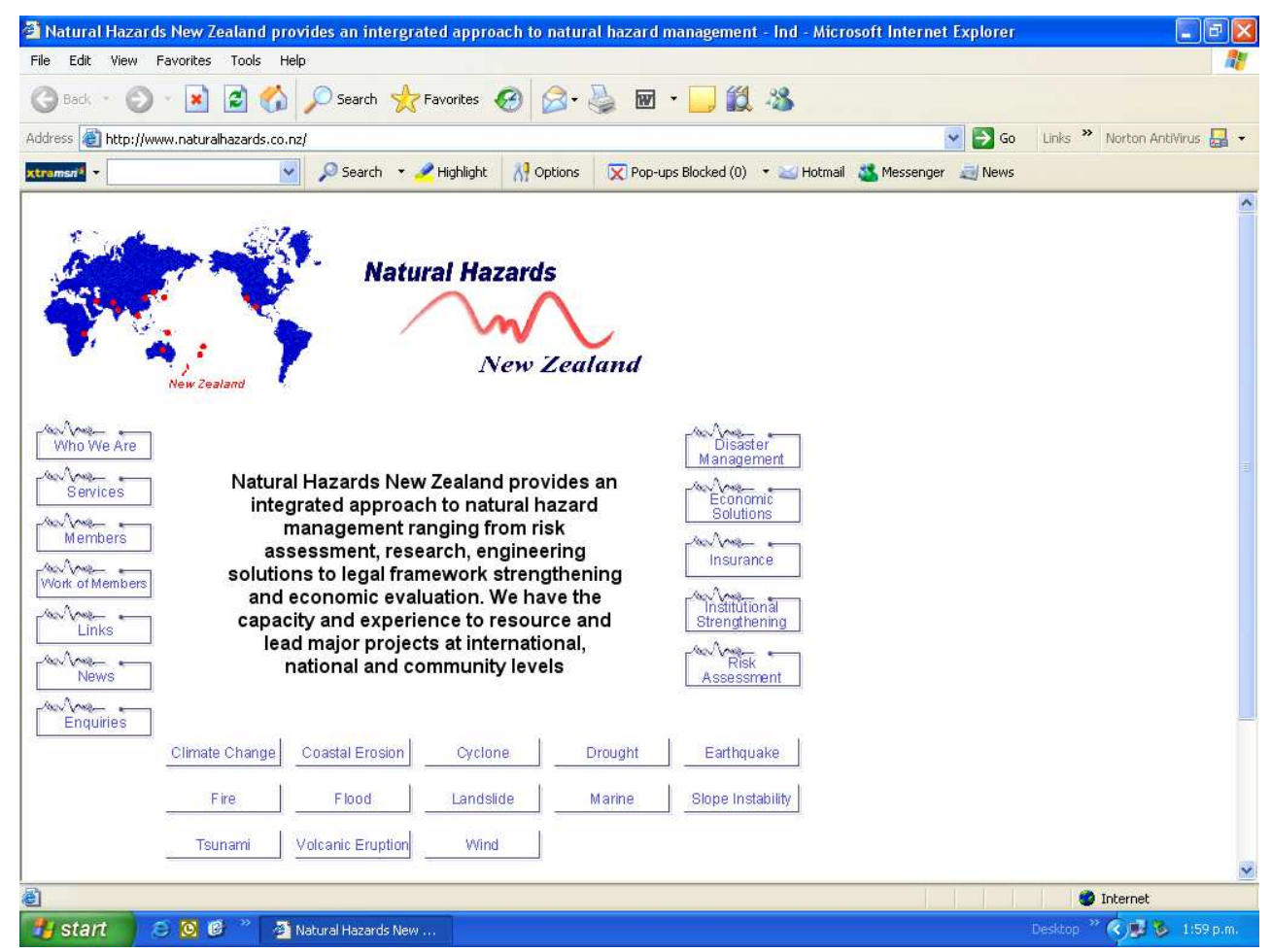

Figure 12. NHNZ's “old” Homepage (source: www.naturalhazards.co.nz).

The old website's navigation focused on promoting individual specialists, as most of its links led visitors to one-page profiles of experts from member organisations who worked on a particular type of service or natural hazard. For example, Figure 13 shows the list of experts' profiles available under the service Risk and Disaster Management. Clicking on one of the names from this page would take the visitor to the one-page profile of that particular expert. 


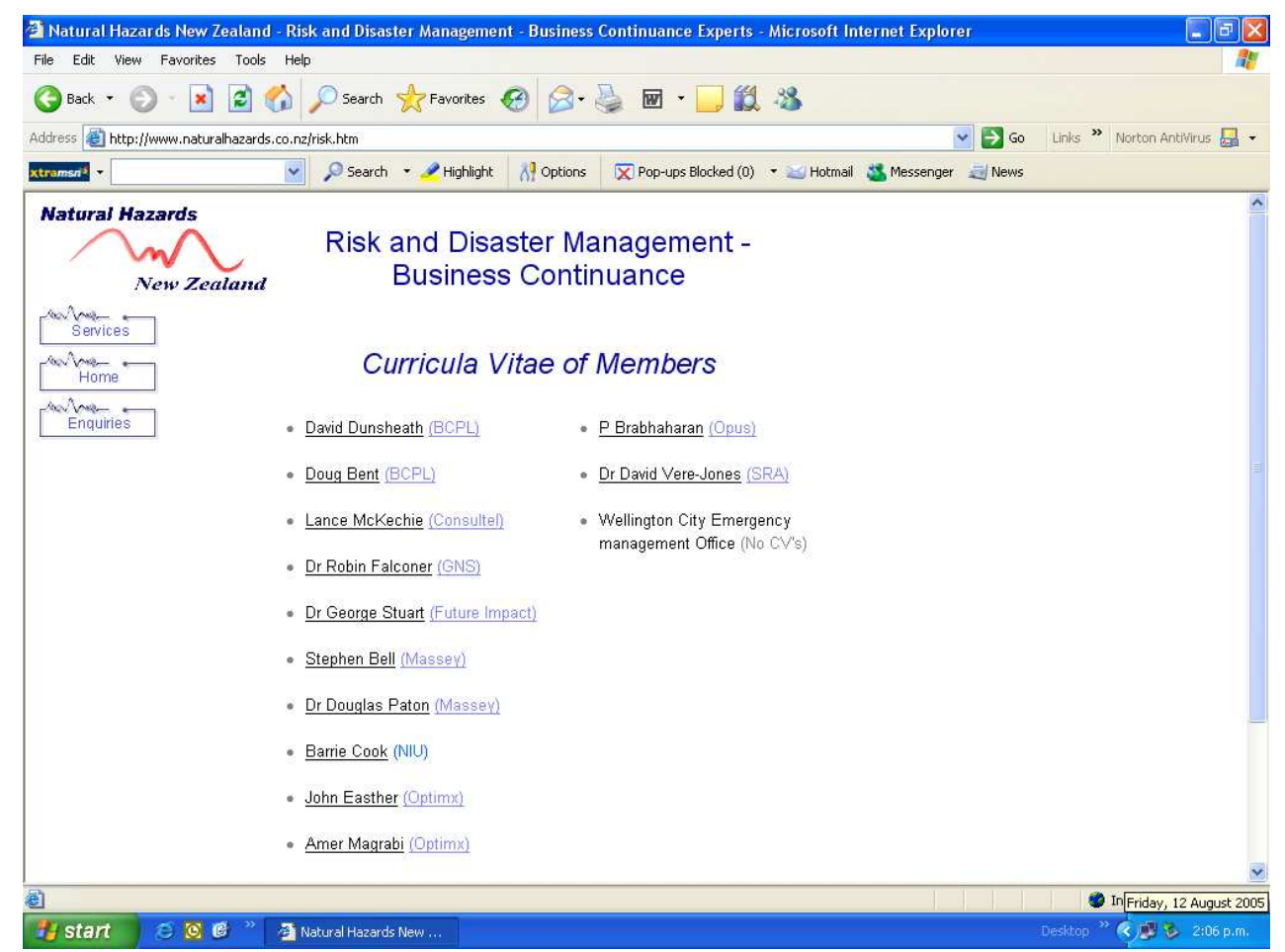

Figure 13. Risk and Disaster Management page from the old NHNZ's website (source: www.naturalhazards.co.nz).

The one-page profile was designed by the cluster. Each profile provided the expert's photograph, contact details, a list of their expertise, a summary of their experience, and their track record. Apart from promoting individuals in the website, these profiles were used for international project bidding, in particular when a bidding team was assembling an Expression of Interest.

The Minutes for the NHNZ meeting held on 16 October 2003 recorded a strong feeling amongst members that the website needed to be reviewed, partly because some of its content was outdated, but mainly to echo the fact that the cluster had matured and accomplished key milestones, such as being shortlisted and winning a couple of overseas projects.

During the cluster meeting of 11 December 2003, a small group was set up - which I volunteered to join - to perform preparatory work on this matter and report back at the first cluster meeting of the following year. This team did not follow through for various reasons, two of which I believe were setting up the team close to the holiday season, and not including an executive as part of the team.

The first cluster meeting of the year was held on 11 March 2005. The urgency of reviewing the website was again raised. One cluster member pointed out that updating and reviewing the content material for the new website would demand "a considerable 
amount of work." From my notes of that meeting, two other points mentioned during the discussion were the facts that the Website should be a valuable tool for the project bidding process and for product promotion.

A second Website Task Group was formed to undertake the review and project management of the website. This time, the two cluster Co-Chairs decided to lead the team. The other constituents of the Website Task Group were two cluster members, the cluster Facilitator, and myself. It took the cluster at least five months to reach the point where they were ready to plan for their new website.

\subsection{The purpose of the new NHNZ website}

The cluster meeting on 26 May 2004 discussed how the new website would fill the following three overarching purposes: 1) be a marketing channel; 2) support the bid process; and 3) showcase cluster products.

The new website was considered a marketing and reference tool for potential clients to learn more about NHNZ. Like the old website, visitors would have access to information about the cluster and a list of its members, the types of services the cluster can offer, and the types of hazards in which they have expertise. In addition, the new website would provide more information about members and their projects, therefore being a channel to promote and showcase the cluster's expertise and capabilities. It was recognised that the website is but one part of the total marketing strategy.

The second purpose of the new website would be to support cluster members while bidding for international projects. The website was to incorporate a restricted section for members, in which explicit knowledge that is usually part of an expression of interest (EOI) or a proposal - such as descriptions of companies, experts' profiles, detailed CVs and examples of previous jobs - would be downloadable. In this way communication transactions between members for required information could be reduced.

There have been instances where a deadline for submission of EOIs closes too quickly and thus gives little time for the lead company to coordinate the rest of the team. Having to request key information for the bid directly from each team member every time a group prepares an EOI or a bid proposal is not an efficient method of working and can be stressful. One example of a situation where the new website could have contributed greatly was while preparing an EOI for one of the reconstruction projects after the 2004 Boxing Day Tsunami: 
Christmas time: no one was around. They wanted all this information. Half of the people were on holidays, me included. But if you want to be able to get something together quickly, you want to get to the website and download it (Interview Task Group member, 04 February 2005).

The product-focus component of the website aimed at promoting specific products that members have developed individually or jointly with others. The potential had been discussed for this space on the website to trigger members to think innovatively and prepare "product packages."

This third component has been the least developed of the outlined purposes for the website because, in the initial stages, the direction the cluster wanted to take was not clear. Nevertheless, two ways the website could fulfil its product-focus purpose have been discussed by cluster members. One option is for the website to include a listing of products, with a brief description of each product and a link to those members supplying it. Alternatively, an e-commerce solution could be developed to sell the products online through the website. The second option is a complex exercise as it would require functionalities such as "shopping basket," electronic payment, and security, but it has potential to become a core business service provided by the cluster in the future.

Defining the purposes of the new NHNZ website was not reached at one moment in time. On the contrary, it was an ongoing process of discussions during cluster meetings, reflection by cluster stakeholders, and dialogue within the Website Task Group that took responsibility for conceptualising the website on behalf of the cluster.

\subsection{Preparing the NHNZ Specifications Document}

A Website Task Group was formed during the cluster meeting held on 11 March 2004. This time the team sprang into action, lead by Co-Chair One who called for a meeting at his premises on 19 March 2004 to start preparing the Specifications Document for the website upgrade.

A few days before this meeting, the cluster Facilitator provided a copy of the Earthquake Engineering New Zealand (EENZ) Cluster Project Brief for their website. This document was a starting point for the team, as it described web pages and some functionality that was designed for EENZ which could be considered for NHNZ's website.

The Task Group firstly brainstormed ideas about what was expected from the new website and raised the following issues: 
- Be able to explain who we are

- The website as a handy online repository of material for the cluster

- Identify the website's audience

- The website as a key source to showcase what the cluster has done

- To be able to view experts CVs from the website.

- The level of detail of content that the website should have

- The maintenance of the site in general, and the CVs and project records in particular.

The Task Group spent time determining some of the key web pages to be included and the navigation of the site. As a group, we agreed on three routes to lead a web visitor to the projects done by the cluster: by hazard, by country, and by service (see figures 14 and 15).

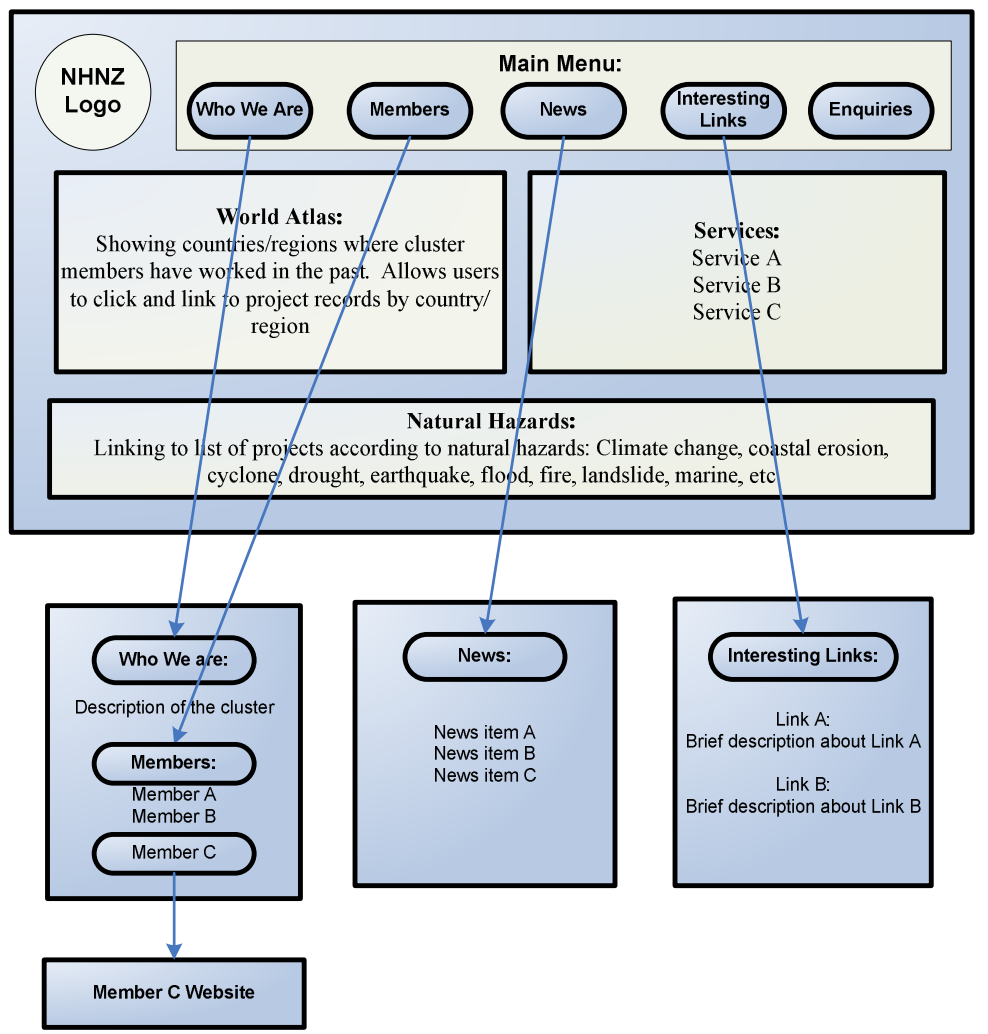

Figure 14. Proposed web pages and navigation from new Homepage. 


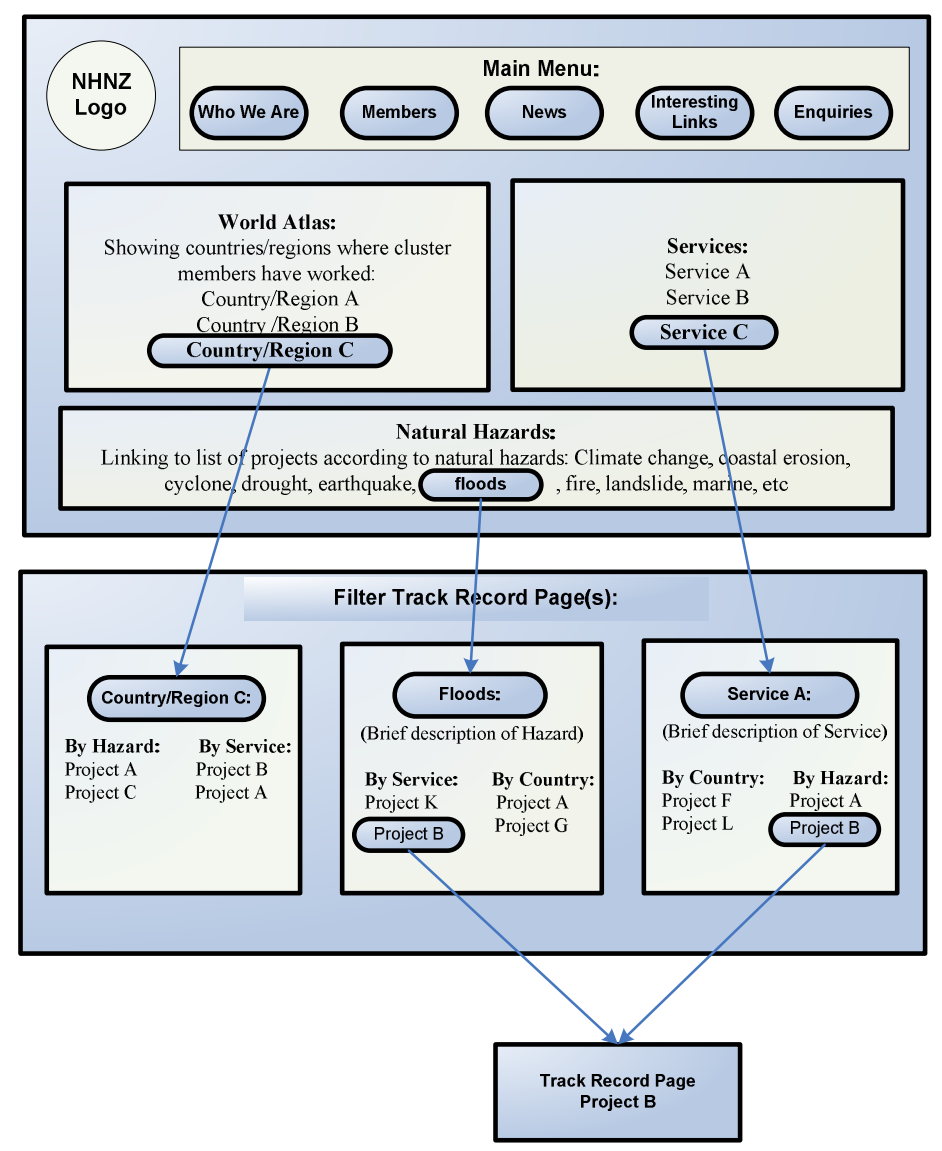

Figure 15. Proposed web pages and navigation to see the projects.

I prepared the first draft of the Website Specifications based on the ideas from the meeting, and this was circulated amongst the Website Task Group via e-mail on 24 March 2004. During the following weeks Task Group members provided detailed feedback to the draft via e-mail. One was the idea of setting up an automated function in the website to enable cluster members to create new online projects and CVs, rather than relying on sending the information to the web administrator to input manually. Although this would save website maintenance costs, the Task Group members were aware that it could bring out other issues such as quality control.

Another Task Group member recommended the use of a database instead of static pages for projects. He argued that, over time, the number of projects would increase and the database would scale more gracefully than static pages without adding overhead costs. Static pages would require the creation of new pages for each additional project and constant maintenance to keep the links working, whereas the database would merely require initial set-up work and after that would generate the pages automatically. A database could also allow search capability to look for projects or experts. 
Showcasing a couple of case studies was also suggested for the new NHNZ website. Case studies could cover the process as facilitated by the cluster from start to finish, thus providing good selling points for NHNZ.

The team members acknowledged that developing such functionality for the website could be a lot of work - and even expensive - but was worth considering. A comment from one Task Group member reminded the others of the importance of keeping the website revision simple:

It is probably important to keep the initial revision of the web site fairly simple for budget and for 80/20 results, but at the same time keep it future-proof by adding other features. (E-mail Task Group Member, 06 April 2004).

Further discussions amongst the Task Group endeavoured to narrow the scope to key features and leave the rest for future stages of the website development.

The team's suggestions were included in the Website Specifications. The updated version of this document was sent to a web developer from one of the cluster organisations for his review and comments. The Task Group met with this web developer on 29 April 2004 seeking further clarification on what the team should expect from website design.

The meeting with the web developer clarified that the team was seeking a simple structure and construction for efficient maintenance of the website, to lessen costs and reduce reliance on a web administrator. It was also agreed that incorporating a database in the website to store information for projects, cluster companies, and individual experts was a good investment.

To make the database a useful tool for the cluster, it was suggested that it work with templates so the system could automatically generate expert profiles, CVs, summaries of projects, or any other type of genre using a standardised format. Templates were not a new concept for the cluster, as they had been using one for their one-page expert profiles in the "old" NHNZ website. However, implementing this approach in the new website would imply further work in creating such templates for the required genres. At the time it was not clear if such a task was within the scope of the Website Task Group:

Need to provide two updated templates for use by members' individual staff, for CVs and projects respectively. These templates need to be userfriendly and stable. Action Point who will revise the CV template? Action Point who will create the Project template? (E-mail Task Group member, 29 April 2004). 
The final Website Specifications were ready by early May in order to be sent to the developers interested in working on this project. The document identified the new NHNZ Website audience (prospective clients, key national stakeholders, cluster members, and general public) and how the website should cater for each of them. The document also provided a table summarising the web pages that should be included in the website, including "Who we are?", "News and events," "Contact us," etc. Finally, desired features and/or functionality were listed in the document, such as search capability, traffic statistics, uploading/downloading information, use of templates, easy printer-friendly pages, etc.

The initial inclination was to keep this job in-house, as the cluster member would have a vested interest in the project succeeding and they would understand the cluster's needs. In a subsequent meeting this argument won, and eventually two cluster members, who had shown interest in developing the website, were invited to tender.

During the cluster meeting held on 26 May 2004 one of the Co-Chairs championing this project reported on the progress made by the Website Task Group. Due to delays, the Website Task Group was unable to bring to this meeting the bids of the two in-house developers interested in the project. Given the fact that the next meeting would take place in August, the cluster agreed to pass on the responsibility of selecting the website developer to the Website Task Group. The Website Task Group would meet to make a final decision on who to contract.

\subsection{Discussion Planning Stage}

The "old" NHNZ website follows the Poster/billboard model (Lawrence, Corbitt, Fisher, Lawrence \& Tidwell, 2000), which involves providing information about the organisation, what it offers, and its contact details. This model is a low-cost approach and is considered appropriate for beginners in Internet commerce. It is a one-way channel of communication between the cluster and its potential clients.

NHNZ has matured and accomplished new milestones, such as being shortlisted and winning a couple of contracts overseas. With these achievements "under their belt" and taking into account the preliminary findings of the Diagnosis Stage, the cluster felt it was time to upgrade the website to something more complex that could provide support for members during project bidding and marketing activities. 
The proposed solution sought by the cluster is a simple web-based information system (Wang, 2001; Yang \& Tang, 2003), where web technology is integrated with conventional information systems - in this case it proposes a database and possibly a content management system. According to Wang (2001), web-based information systems are becoming popular due to the convenience of carrying out business processes online and the importance of electronic commerce.

The Website Task Group took responsibility for conceptualising the new NHNZ website. The Diagnosis Stage involved understanding the purpose of the application and its desired characteristics and functionality. This stage demanded dialogue, learning about ICT and website design, and reflection by the Task Group over a period of time. The output of this stage was the Website Specifications document, which detailed the requirements for the new website. This document was then distributed to web designers interested in developing the project.

The Website Specifications document produced by the Task Group is the first document that is usually developed in systems development, in order to scope the project and detail the user requirements (Kliem, 1992). The Task Group - comprised by future users of the system, management and the researcher, who carried out the role of systems analyst - conceptualised what was expected from the system.

It was interesting to observe some emerging themes at this early stage of the project that were similar to those raised by Braun (2003a). For example, the levels of knowledge about ICT and website design, and how to tackle any shortfall, were aspects experienced in both studies. The difference however was in the approach followed by the groups. The NHNZ Task Group - who appeared to be more comfortable with the technology than those in Braun's study - were keen to learn more and proactively sought clarification about website design by meeting with a web developer.

Given the several purposes that the new NHNZ website is expected to fulfil and the various features it will encompass in both public and private areas, it was suggested that it be thought of as a portal. A corporate portal uses browser interface and web standards to develop a solution that offers communication, collaboration, and access to company information from a single point to employees, business partners, and customers (Turban \& King, 2003; Detlor, 2000).

Corporate portals have been described as the "natural evolution" of intranets (Watson \& Fenner, 2000; Dias, 2001) as they have the potential to include links to other systems, 
internal and external data sources, and the Internet. The portal model offer cluster members, business partners, and customers a focal point for their interactions with NHNZ.

Sharing and reusing knowledge is a common practice in NHNZ, even at this early stage of the intervention. For instance, EENZ's Cluster Project Brief was a starting point for the team, as it described web pages and some functionality to be considered for the new website. This was also an example of knowledge sharing across clusters.

Another example of knowledge reuse was the one-page expert's profile that the cluster designed for their "old" website. The cluster found this genre useful for marketing and project bidding and wanted not only to improve its features with web-based and database technologies, but to create a similar template for their project summaries.

Finally, during the Diagnosis Stage the Task Group identified some of the genres they required for project bidding. In addition to the already available Expert Profile genre, the team proposed project summaries and case studies as new genres to be created. The Task Group also discussed the functionality that database and Internet technologies could bring to genres, such as being able to link them and being dynamically created from templates.

As Website Task Group members become familiar with Internet and database technologies, they became increasingly interested in the possibility of digitising and incorporating into the system some of the genres they currently use in project bidding. 


\section{Chapter 8: The Development Stage}

The Development Stage was the longest and most complex stage of this study. It started in late April 2004 when the team selected the web developer who was going to work on the new NHNZ portal, and concluded in November 2005, after the official launch of the portal by New Zealand's Prime Minister, the Right Honourable Helen Clark.

This stage was also the most "hands-on" of the study, where two Task Groups (the Website Task Group and later the Content Task Group), on behalf of the cluster, interacted with the web developer to build the website according to the Website Specifications Document produced at the Diagnosis Stage. The cluster Facilitator, the two cluster Co-Chairs, and I participated in both Task Groups. Other cluster members and their staff and a Positively Wellington Business (PWB) Liaison Official joined these Task Groups at various times.

Most of the data collected for this stage came from the e-mails exchanged by the Task Groups while working on the project. This was complemented with interviews, documents produced by the Task Groups, cluster Minutes, observational notes taken during the Task Groups meetings, and the entries from my diary.

Similar to the Diagnosis Stage, my analysis focused on examining the events associated with the project over time. Again I applied Miles and Huberman's (1994) matrix to systematically display the data in chronological order, which enabled me to write the "story" of the Development Stage in this Chapter. I also carried out open and axial coding of the data collected during this stage, to develop categories that fed into the interpretation of findings detailed in Chapter 11, 12 and 13.

The web developer followed the evolutionary prototype approach to create the NHNZ portal. Prototyping is a dynamic process that involves building a model based on business requirements. The model is then reviewed by end users, who suggest changes that are usually incorporated by the developer to further refine and enhance the prototype (Haag, Cummings \& McCubbrey, 2005). In evolutionary prototyping, several cycles of development and user evaluation would take place before the final system is achieved (Baskerville \& Stage, 1996).

In our case, the prototype was mainly reviewed by the Website Task Group and was refined until it became the NHNZ Portal. The website content was mainly reviewed by the Content Task Group. Prototyping fitted nicely within the action research framework, 
as the researcher was able to identify and record each iteration following a planning, changing, and evaluation sequence.

The overall project involved five iterations between the Website Task Group (and later the Content Task Group) and the web developer. Each of these iterations began with a document addressed to the web developer, in which the Website Task Group indicated the changes to be made to the portal's prototype. During these iterations, meetings between the Website Task Group and the web developer were held to assess the progress of the project. The iteration concluded with a document outlining further instructions to the web developer on how to proceed. This document would be the start of the next iteration.

The final version of the NHNZ portal was launched on 10 November 2005 during the official commemorations of the cluster's fifth anniversary. Although launching the new NHNZ portal was considered a huge achievement and the milestone for this stage of the $\mathrm{PhD}$, cluster executives were of the view that the new portal should undergo further work to make it a more valuable tool in the marketing and project bidding processes than it is at present. The following is a description of the events that took place during the Portal Development Stage of this study.

\subsection{Preceding Step: selecting the web developer for NHNZ Portal}

The preceding step involved a set of activities to select the web developer at the beginning of the Portal Development Stage. It is not considered an iteration of the portal development process as it was a transition between what was decided at the Planning and the actions taken at the Development Stages of this study.

\subsubsection{What happened in the Preceding Step}

In the portal Diagnosis Stage, a decision was made that tender for the portal would be in-house, given the fact that the IT Departments of two cluster members were interested in the project and were charging a competitive rate for the job. The Website Task Group distributed the Website Specifications document to the bidding parties and they in turn provided their respective proposals for consideration.

The Website Task Group met on 20 May 2004 to review the proposals and decide which developer to recommend in the upcoming cluster meeting on 26 May 2004. The team needed further clarification from the developers. Both developers assumed that the 
cluster only wanted to store PDF files of projects and CVs, and consequently the option of a complex database was not considered in their quotes, so new costs were provided. I produced a combined matrix with the answers from both developers and submitted it to the Task Group the day before the cluster meeting.

Since the Website Task Group was unable to complete the assessment for both quotes, the NHNZ Minutes for this meeting recorded the agreement for the Website Task Group to oversee the rest of the website development and to ensure that ongoing hosting and maintenance costs be kept to a modest level.

The Website Task Group selected the developers to perform the work. During the meeting with the successful candidate, the developers showed their organisation's staff profile database, and suggested that a similar system could be implemented in the NHNZ portal. The attractiveness of this database is that other people within the organisation could tap into it to find out who is an expert in a particular field, learn about what projects the person has been involved with, and get their contact details.

A feature that impressed the Website Task Group about this database was the fact that details of an expert could be exported to two predefined printable genres that are fed from specific templates of the database. In principle, the cluster could create templates for the various $\mathrm{CV}$ formats required by the different development agencies such as World Bank, the Asian Development Bank (ADB), and NZAID.

The Co-Chairs and the cluster Facilitator received a final contract from the successful developers on 16 June 2006, detailing each activity the developers would perform and its cost. The Co-Chairs and the cluster Facilitator reviewed the quote and accepted it.

\subsubsection{Discussion Preceding Step}

The activities in the Preceding Step followed a typical tender process; even the request for additional information from both developers is expected when tendering.

Although the Website Task Group members were regular users of Internet applications and had a good understanding of the technology, it took them some time to come to grips with how to conceptualise the NHNZ website and how to assess both proposals in terms of what the cluster wanted.

In this transition step no new knowledge for project bidding was created, shared, or reused as such. However, it marked the beginning execution of the development of a tool that will support knowledge transfer. It could also be argued that the Task Group 
learnt a little more about tendering an IT project. The experience could help to better organise the next tendering process.

The web developers were keen to build a database to handle the cluster's CVs based on a system they had developed in-house for their own staff profile management. Using the framework of types of knowledge at firm and cluster levels proposed by Tallman et al. (2004), the system already in place at the website developers' organisation is an example of component knowledge. This system is highly technical as it is explicit, tangible, and provides step-by-step instructions for use. It will be easily replicable for the cluster and thus transferable, which was reflected by the developers' comments regarding the use of similar tables and coding for the NHNZ database.

Although a cluster member was paid as a contractor to build the portal, all parties agreed that payment was below the market value for developing such a system. The price paid covered the developers' time on the project. The cluster was granted access to the expertise and experience of a team with a proven solution.

\subsection{First Iteration: developing the first prototype of NHNZ Portal}

The first iteration began after the meeting with the developers who won the NHNZ contract on 04 June 2004, and concluded shortly after the first prototype was presented to the cluster during the Annual General Meeting on 26 August 2004. This iteration was characterised by identifying the fields for the database component of the portal, by the development of key web pages, and by providing the feedback to the developer.

\subsubsection{What happened in this First Iteration}

On 8 June 2004 the lead developer sent a document with the data model he used to develop his company's staff profiles, which he suggested using as a base for Experts Profile and Company Profile. The model defined the entities and fields to collect pertinent information, and explained the properties of each field.

The lead developer also commented on the need for further discussions with the Website Task Group to define the Project Sheet, which is the other genre to be created. I was aware that two of the group were overseas on business trips and therefore requested more time to send back instructions on how to proceed. 
On 23 June 2004 I reminded the Website Task Group to provide feedback. One of the respondents attached examples of description sheets that his organisation had prepared for projects. He proposed using these as a base to create the cluster's Project Sheet:

I have attached a couple of project description sheets from work we have done. We may need to develop them further from here to suit Natural Hazards and our target cliental. (E-mail cluster Co-chair One, 23 June 2004).

On 25 June 2004 I passed on to the lead developer those fields that were confirmed by the Website Task Group for Company Profile and Project Sheet. Although progress was made in collecting feedback, further agreement was needed.

On 12 July 2004 the lead developer asked to meet with the Website Task Group to present the first prototype. A meeting was arranged on 23 July 2004 but was cancelled at the last minute as most of the Website Task Group team were attending another cluster Task Group met to discuss a potential business opportunity in Turkey.

The Website Task Group met with the web developer on 30 July 2004. The developer showed the web pages, menus, and forms he had created to manage the portal's content. Some areas in the prototype were sketchy as the developer needed information from the team - in particular details related to the Expert Profile and Project Sheet. Nevertheless, the Website Task Group was excited to see the system taking shape.

Three levels of access to edit information in the website were agreed: Standard User, Primary Contact, and Website Administrator. The Standard User would have access only to edit their information from Expert Profile. The Primary Contact would have access to their company details as well as to the Expert Profiles of the people working in their organisation and therefore is responsible for updating the Company Profile, adding/deleting/modifying the Project Sheets of their company, and if necessary editing information from their colleagues' Expert Profiles ${ }^{14}$. Finally, the Website Administrator would have access to all the pages for content maintenance and updating.

The Website Task Group agreed to show the prototype at the Cluster Annual General Meeting on 26 August 2004. Therefore entering some content into the pages became a priority. I met with one Website Task Group member at his office to prepare the content. We reused the content from the old NHNZ website and the cluster's brochure and marketing PowerPoint presentation.

\footnotetext{
${ }^{14}$ Individuals should be encouraged to review and edit their own Expert Profile regularly.
} 
On 6 August 2004 the lead developer notified Co-Chair Two of the status of the prototype before he took one month's leave. The portal had become this developer's responsibility and it appeared it was not going to be picked up by anyone else in his team. This posed another delay to the portal's development, adding to those already caused by the Website Task Group's late feedback and cancelled meeting. Delays from both sides kept occurring throughout the Portal Development Stage.

The Minutes of the NHNZ Annual General Meeting on 26 August 2004 recorded that members were informed of the website developer selected. Members were told that the Website Task Group decided to develop a more advanced database system than originally considered, which could be used to manage the site's content.

I was asked to lead the demonstration of the prototype to the cluster. I showed the cluster the navigation of the prototype as well as examples of two Company Profiles, one Expert Profile, and Project Sheets. Other Website Task Group members made contributions to the presentation and participated in the discussion that followed. I collected feedback from cluster members during the session.

While examining the Project Sheet examples, members commented on the usefulness of creating another template based on how the World Bank asks bid applicants to describe their projects. Cluster members acknowledged that the information requested by the World Bank encompass details about a project that members may not want to disclose publicly. The suggestion of another way to portray a project was evidence that a "private" project sheet should also be considered.

The cluster expected to have a final version of the portal for review at their next cluster meeting. It was also agreed that, once the new website was completed, the cluster would organise an official launch function event.

On 6 September 2004 I informed the Task Group that the web developer had returned from leave and we had agreed to meet on 9 September 2004 to discuss the prototype. I invited members of the Task Group to attend but only Co-Chair Two, who works in the same organisation, was able to attend.

Prior to our meeting, I e-mailed the developer the Feedback Document. The document gathered all the feedback put forward at various instances: the suggestions made by the Task Group to the developer during the meeting held on 30 July 2004; the prototype demonstrated during the cluster meeting on 26 August 2004; what I noticed in terms of presentation (like not listing the cluster members in alphabetical order); and 
programming errors I detected while populating the portal. This document marked the conclusion of the first iteration of this study's Development Stage.

\subsubsection{Discussion First Iteration}

The Website Task Group focused its attention on three genres they wanted to digitise and make readily available through the new portal: Expert Profile, Company Profile, and Project Sheet. The Expert Profile and the Project Sheet were identified in the Diagnosis Stage of the study as part of the cluster's selection of genres used in the project bidding system. Company Profile was a new genre proposed to be developed in this iteration to describe each organisation that is a cluster member.

From the genre systems analysis applied to Expert Profile and Project Sheet during the Diagnosis Stage, we learnt that both genres employed paper-based or PDF document as their medium of communication. These two genres are also highly- structured and follow well-defined formats. The time frame to have both genres available tends to be "urgent," as they are needed when preparing the Expression of Interest (EOI) or when building relationships with clients. Given these characteristics, it was no surprise to see that the Website Task Group selected them as ideal candidates to start the digitisation process of their genres. The cluster also proposed considering the creation of another genre, the "private" Project Sheet.

The Website Task Group also started to conceptualise social rules around the proposed genres by proposing three levels of access to edit their content: Standard User, Primary Contact, and Website Administrator.

The iteration showed evidence of the readiness by two company members to share some of their knowledge for the benefit of the cluster. The Data Model Document confirmed the developers' commitment - on behalf of their company - to share with the rest of the cluster their knowledge on how to structure the database, so the cluster could reuse these principles to digitise Expert Profile and Company Profile. Another cluster member offered the genre his company uses to prepare the cluster's own Project Sheet. Both contributions helped the Website Task Group define the fields needed in the database, thus avoiding "reinventing the wheel."

The website Task Group's momentum was low at the beginning of the iteration. It took a few weeks for the team to review the suggested fields for the database. This in turn delayed the web developers in creating the prototype. 
Despite the gaps in the specifications, the lead developer was able to produce the first prototype for the Task Group to view and interact with. During the meeting with the web developer on 30 July 2004 much progress was made by asking complex questions while showing the prototype to the Task Group. This allowed the team to visualise problems, discuss them, and agree on solutions.

\subsection{Second Iteration: developing the second prototype of NHNZ portal}

The second iteration began on 9 September 2004 with a meeting to discuss the feedback collected for the first prototype, and concluded on 17 January 2005. This iteration was characterised by a shift in how the Website Task Group wanted the Expert Profiles and the Project Sheets to be displayed online and in print, thus causing major modifications to what was developed for the first prototype. Delays caused by all parties slowed down the development of the Portal.

\subsubsection{What happened in Second Iteration}

On 9 September the lead developer, the cluster Co-Chair Two, and I met to discuss the Feedback Document. We explained to the developer that cluster members commented on the usefulness of creating a Project Sheet based on World Bank specifications that only members could view. The Co-Chair showed a copy of a World Bank template he used in a recent bid ${ }^{15}$. The developer pointed out that this was additional work.

I wrote to the rest of the team on 14 September 2004 briefing them about our meeting with the developer. The e-mail listed the key issues on which the web developer wanted the Website Task Group make decisions. A reply from the cluster Co-Chair One informed the rest of the team that he and Co-Chair Two were going away on a business trip; therefore any progress on the portal was postponed until their return. Co-Chair One also commented that the cluster should focus on developing a system to automatically generate only the "public" Project Sheet at this stage, and make available an unfilled form following the World Bank format to be shared by cluster members for the "private" Project Sheet ${ }^{16}$.

\footnotetext{
${ }^{15}$ I was allowed to keep this template for my records. I also collected examples of CVs from the web developer and the Co-Chair. I showed both sets of genres to the Website Task Group at a later meeting.

${ }^{16}$ I would revisit the "private" Project Sheet with the cluster Co-Chairs at a later development cycle outside the timeframe of this study.
} 
The Website Task Group met with the developer on 1 October 2004. For this meeting I prepared a table comparing each of the items from our requirements document with the actual contract with the developers and with what had been already delivered so far. This table was useful to view progress made in the portal.

Several points regarding the Expert Profile were finalised, such as making it available in PDF format to the public, and stipulating that the layout would be similar to that used on the old website with some additional fields. It was decided there would be two ways for a visitor to navigate the Project Sheets: by region and by country. Under "by regions" the visitor would click on a world map divided into regions, while visitors following the "by country" navigation would choose a country from a list.

I prepared the draft of a second Feedback Document summarising what was agreed in the meeting. A key component of this document was a table listing all the fields to be considered for the Expert Profile. I sent the feedback document to the Website Task Group for verification. With exception of one member, by 26 October 2004 - almost a month after the team met with the web developer - the group had not responded on whether the feedback document could be sent to the web developer.

To help the team return its focus to the project, I updated the draft of the feedback. I also included another table listing the fields for the Project Sheet based on our meeting of 1 October 2004. Two Website Task Group members replied to this second reminder and both validated the feedback document.

I met with the web developer to discuss the final version of this Feedback Document on 5 November 2004. I brought a printout of the template for the Expert Profile currently being used by the cluster in the old NHNZ website so that he could start creating the PDF template. We also discussed the properties for each field in the Expert Profile and the Project Sheet that needed further clarification. The developer was to make further modifications to most of the tables and forms developed for the Expert Profile in the first prototype to comply with the revised fields. He aimed to complete the second prototype by 25 November 2004; not in time for the next cluster meeting scheduled for 18 November 2004.

During the cluster meeting, I reported on the progress made on the Portal. The cluster was informed about the decision of reusing the one-page CV template created for the old website (i.e. Expert Profile). The Minutes for that meeting recorded the expectation 
to have the Portal completed and ready for final approval by the next cluster meeting scheduled in early March.

On 26 November 2004 I e-mailed the web developer to inquire about his progress with the prototype. I also informed him that I would be away until 9 December 2004. Later that day the web developer released the second prototype. This prototype allowed the automatic generation of Expert Profile in PDF based on the information held in the database. This aspect of the NHNZ Portal was a huge improvement from the old NHNZ Website, which held only static PDFs about the experts. The one-page Expert Profiles were to be used by cluster members for project bidding, but were also to be accessible to users visiting the public area of the NHNZ Portal.

Christmas and the end-of-year holidays arrived and this put a halt to the progress on the Portal. I talked with the cluster Facilitator in January 2005 about the lack of progress and my concern that the Portal would not be completed by March as expected by the cluster. The Facilitator suggested the two of us meet with the developer and see what we could do to kick-start the project once again. The Facilitator also suggested inviting Co-Chair Two, who works in the same organisation. He agreed to attend briefly.

I arranged a meeting with the developer for 13 January 2005. During the meeting attention was focused on the layout, design, and content of the web pages. Changes were requested for the wording used on some of the buttons and links. Comments and suggestions were recorded which complemented my notes in order to produce a third version of the Feedback Document, which was handed to the web developer on 17 January 2005.

The feedback document reflected the need to review the content already entered in some of the Portal's pages. There were also indications that other web pages - defined in the Website Specifications Document - were to be created, such as "Overseas Visitors," "News and Events," and "Interesting Links." The Feedback Document marked the conclusion of the second iteration of this study's Development Stage.

\subsubsection{Discussion Second Iteration}

The Task Group faced one factor of project failure, namely a weak commitment by the project team (Kappelman, McKeeman \& Zhang, 2006). Although acknowledging that the Task Group has other higher priority working commitments than the portal development, the project suffered delays and backtracking as a result of not dedicating sufficient time to reflect on the social rules around the information to be contained and 
the format of the outputs for the genres during the first iteration. In this iteration the Task Group was forced to clarify aspects of the Portal that until then had been sketchy, in order to move the project forward.

However it can be argued that the prototype approach tried to address this issue of insufficient requirement definition by developing a rudimentary system to involve users in the design of the system (Baskerville \& Stage, 1996). Therefore the work undertaken to define fields in the online forms and the templates in the previous iteration should not be considered wasted.

A key focus of this iteration was finalising the Expert Profile and the Project Sheet. The Task Group resorted to some of the genres that individual cluster members had used in previous EOIs and tenders as a starting point. The cluster Facilitator and a Task Group member provided the templates to be studied from their personal repositories. These are further examples of component knowledge that was transferred to the cluster (Tallman et al., 2004).

Finally, the Portal was emerging as the central repository proposed by Markus (2001) to encourage knowledge reuse. The Portal would make available to the cluster community knowledge about individuals' expertise and companies' capabilities.

\subsection{Third iteration: Developing the third prototype of NHNZ portal}

The third iteration began on 17 January 2005, with the feedback collected from the second prototype, and concluded on 15 March 2005. This iteration was characterised by a renewed commitment to providing feedback and contributing to the development of the Portal by some Website Task Group members who had been relatively passive in the previous iteration. Most of the coordination and decision-making process for the Portal's development took place via e-mails, as no formal Website Task Group meeting was arranged for this iteration.

\subsubsection{What happened in the Third Iteration}

In the following days the developer worked on an alternative look for the Portal, based on the Feedback Document and the input he collected during the meeting on 13 January 2005:

I've put together an alternative look for the NHNZ website [...] Could you and the guys take a look and see if it is more along the lines of what you were thinking? (E-mail Developer, 21 January 2005). 
Both versions needed further design work, but the developer wanted to know which one was more in line with the cluster's preference. I invited the Task Group to visit the links where the two versions of the Portal's look were located.

I received extensive input about the Portal's look via e-mail from two Website Task Group members who had been relatively quiet in the previous iteration. One of them is not based in Wellington, so he made an effort to prepare a mock-up along with his comments to compensate for his inability to meet face-to-face with the team.

On 1 March 2005 I met with the web developer to discuss how to proceed with the design of the Portal based on the input from these two Task Group members and discussions with two other members over the phone and face-to-face. I reported back to the Website Task Group about this meeting with the web developer and confirmed that the changes would be part of a third prototype to be presented at the next cluster meeting on 10 March, 2005.

One Website Task Group member commented that focus should now be directed the wording of each of the key pages that make up the NHNZ Portal (i.e. Home Page, Hazards page, Services page, and Products page). For him, the text needed to be packed with keywords and phrases relevant to the topic matter in each page. Unfortunately he was unable to lead this task:

Can anyone else please take the lead to guide Maria? I'm very hard pressed to personally contribute at this stage. (E-mail Task Group member, 04 March 2005).

Although content had been entered in the Portal for illustrative purposes, no actions were taken toward its quality control during this iteration. This issue would be addressed later in preparation for the Portal's launch. Examining the content was a demanding task and at this stage no one volunteered to lead it.

On 3 March 2005 I tested the content management system of the Portal and entered some text for the Services section based on the cluster's PowerPoint presentation. Despite encountering some programming glitches I found the experience positive. It was good to see how the system was going to make content updates easy.

For this version of the NHNZ Portal some web pages, such as News and Events, Interesting Links, and Who We Are, would not be updated through the content management system. During my time with the Website Task Group I volunteered to create and update these pages. 
Co-Chair One wanted some Expert Profiles, Company Profile, and Project Sheets to be entered into the system to show to members during the cluster meeting on 10 March 2005. An employee from his company contacted me on 4 March 2005 to assist in testing the database for this purpose.

I met with the tester on 8 March 2005 and gave her instructions on the type of information she needed to collect, such as the logo of her company, photos of experts, descriptions of projects, and a summary about her company. I also explained to her how to enter the information. I registered the tester as the "Primary Contact" company and I gave her the URL address where the prototype was located.

The testing of the prototype highlighted errors in the routines and forms when executed by a Primary Contact. The tester also identified aspects of the system that could be improved, such as the navigation between forms to make data entry easier.

I contacted the Website Task Group members to report on progress made on the Portal's look. I invited them to check the new look and requested comments. I set up their accounts and passwords with which to log in and test the system by entering details about their respective companies. Finally, I made it clear that I needed the guidance of at least one of them to write good content.

The reply from one of the Website Task Group member with IT experience focused on the issue of ensuring consistency of the web design across various web browsers. To illustrate this point, he sent screenshots of how different the Portal's Home page looked when viewed using other web browsers (Safari, Opera, MSIE, and Firefox) as opposed to Internet Explorer and Netscape. He then offered to help in fine-tuning the Portal's web pages. This was an opportunity to have a member of the Website Task Group take responsibility for an aspect of the project, especially when this member was keen to apply his technical knowledge for the benefit of the rest of the cluster.

A second Website Task Group member gave feedback about his experience when entering information about his company and his profile. Like our main tester, he indicated that many of the forms needed to be better explained. This could be addressed with clear instructions embedded in the forms and a User Handbook to guide the firsttime user. In addition, this member raised the need to improve the "friendliness" of the system:

\footnotetext{
${ }^{17}$ She would have access to edit/add/remove information related to her company.
} 
One thing that irritated me was that when I went outside of secure screens to view my changes in the public areas, the only way back again was to log out and then log back in - rather tedious so probably should be improved. (E-mail Task Group member, 10 March 2005).

The cluster Co-chair Two introduced me to one of his work colleagues, who was to contribute to the Portal's development as an extra team member:

I would like to introduce you to [new person] who is now working in [Co-Chair Two's Company]. I have asked [new person] to take over from me on leading the [Company] contribution to the future development and input to the site. I will still contribute but with me being away so often it's better to have a more consistent and reliable person to make sure that things get done. (E-mail cluster Co-chair Two, 09 March 2005).

The cluster meeting held on 10 March 2005 focused on a special presentation by two members who took part in a scoping mission funded by NZAID to Aceh, a region in Indonesia badly affected by the 2004 Boxing Day Tsunami. Nevertheless I was able to deliver a quick progress report on the NHNZ Portal outlining the third prototype. The cluster members wanted to know when the website would be ready for companies to start entering data and when it would be ready for launch. My reply was that the Portal was nearly completed in terms of structure and pages but needed further testing.

The next day I prepared an extensive e-mail to the Website Task Group and the web developer. I offered to review the October 2004 and January 2005 Feedback Documents to check which of the items listed then had not been addressed by either the Website Task Group or the web developer. It was essential for the Website Task Group to make final decisions on some aspects mentioned in those Feedback Documents. Based on observations made during the cluster meeting regarding some functionality missing in the Portal ${ }^{18}$, I also suggested revisiting the contract with the lead developer and checking which items of the contract had not yet been delivered.

On 15 March 2005 the Website Task Member who had committed to standardising the Portal web pages completed this task. Now the Portal was working well in the following browsers: Windows XP (Mozilla Firefox, Opera, Internet Explorer); Mac OS X (Mozilla Firefox, Safari, Opera, Internet Explorer); and Linux/Fedora Core 3 (Mozilla Firefox, Konquerer).

\footnotetext{
${ }^{18}$ For example, a cluster member asked about the search functionality, which was listed as part of the Specifications Document for this Portal.
} 
That same day I delivered an updated version of the Feedback Document to the web developer and the Task Group. This document marked the conclusion of the third iteration of this study's Development Stage.

\subsubsection{Discussion Third Iteration}

In this iteration the look and feel of the Portal was reviewed and was standardised to the most common web browsers. It also focused on reviewing what had been developed for the Portal, rather than working on new features, by carrying out usability testing. Usability testing is an empirical method where a small group of users are asked to carry out tasks which the system has been designed to do (Agawarl \& Venkatesh, 2002; Prasse, 1991). For instance, the tester and one of the Website Task Group members tested the database, while I tested the content management system. As users, we identified shortfalls that did not make the Portal user-friendly and provided our views to the web developer on how we would like it to work.

Testing the portal highlighted how important it was to produce good instructions in order to capture the right knowledge. For example, many parts of the forms that collected information about the experts were not clear on the type of information needed to produce the Expert Profile. As our main tester indicated, there was no "list of questions" for the data entry person to guide their preparatory work.

This shows that the forms created in the previous iteration - although useful to produce the Expert Profile, the Company Profile, and the Project Sheet - would need to incorporate additional instructions to assist the end user. To complement the work on the forms, the Task Group, on behalf of the cluster, would have to define procedures on how users should engage with the Portal. Both of these activities would produce what Tallman et al. (2004) defined as component and architectural knowledge about the use of a technology that is new, thus enhancing the cluster's knowledge-base.

The Website Task Group did not meet with the web developer in the third iteration; instead the Website Task Group channelled their feedback and queries for the web developer through me. I used the e-mail extensively to communicate with the web developer and only met with him once, mainly to discuss the Portal's look and feel.

During the cluster meeting on 10 March 2005, one member asked about the search engine functionality that was part of the requirements for this project. This reminded us of the importance of revisiting the Specifications Document and the Contract to verify what had been agreed with the web developer. 


\subsection{Fourth iteration: Developing the fourth prototype of NHNZ portal}

The fourth iteration began on 15 March 2005 with the feedback on the third prototype, and concluded on 28 July 2005 with the presentation of the prototype during the cluster's annual general meeting. This iteration was characterised by a focus on the Portal's content and solving remaining technical issues. A review of the contract with the developer showed that some expected Portal functionality was not included and thus an additional contract was signed to cover this shortfall. Some decisions were made by key people instead of going through the process of calling for a Website Task Group meeting. A second round of testing took place, which provided ways to improve the system's usability. By the end of this iteration, the Website Task Group was disbanded and a Content Task Group formed in its place to oversee the Portal's content.

\subsubsection{What happened in this fourth iteration}

The fourth iteration initiated with a meeting between two members of the Website Task Group and me on 24 March 2005. In this meeting we listed all the pages in the NHNZ Portal and assessed their content. I brought print-outs of NHNZ's marketing strategy and profile to reuse words and phrases for the Portal.

The main menu of the Portal listed 11 static web pages: "Home Page," "Our Services," "Our Products," "By Hazards," "By Region," "Members," "Who We Are," "Overseas Visitors," "News and Events," "Interesting Links," and "Contact Us."

Apart from these pages, there were others created dynamically by the content management system in the Portal: the Company Profile page for each of the 33 cluster members; 11 pages for the services provided by the cluster; and 13 pages for the types of hazards. There are also a non-specified number of pages for products ${ }^{19}$.

We agreed that the content for the Company Profile should be the responsibility of the individual cluster member. Finally, we focused on defining the content needed for each of the Services and Natural Hazards pages.

One of the Website Task Group members offered to prepare the description for the Hazards pages. She then e-mailed a draft to cluster members for feedback. Like me, this Website Task Group member had difficulties in obtaining feedback:

\footnotetext{
${ }^{19}$ The pages for products had not been developed at this time; eventually pages for three products were incorporated in the first Portal version. The task of looking at more products will be left for future Portal development.
} 
I have not heard from anyone else (other than [cluster member]) regarding the hazards pages, but the deadline I put on that is tomorrow, so I'll wait to see if any more comments come in, and keep you posted. Again, it is hard to make progress if others don't give any feedback! (Email Task Group Member, 19 April 2005).

As this Website Task Group member and I discussed informally, it was "a long shot" to ask cluster members to be proactively involved in producing feedback at this stage. Further refinements would be made to these pages on a later cycle.

On 29 March 2005 I e-mailed a progress report to the Website Task Group in which I outlined a tricky issue. I had recently compared what had been delivered by the web developer up till then against what was agreed in the contract. A couple of content management functions and some web pages mentioned in the Specifications Document were not clearly stated in the contract, and therefore the developer was not obliged to deliver them ${ }^{20}$. Although the developer had been accommodating and included some of these functions in the current agreement, more complex routines were to be negotiated in a second contract.

Given the number of decisions regarding the content and the technical components of the Portal, the Website Task Group agreed to meet on 11 April 2005. Coincidentally, the Website Task Group member based out of Wellington was in town visiting clients and managed to attend our meeting.

The meeting allowed the Website Task Group to focus on: making decisions about the Home Page; discussing the development of the remaining static pages; and defining new action points. Apart from those features that were not included in the original contract, the Task Group identified three others they would like to see in the Home Page: labels under the hazards icons, hot links, and a world map. The Task Group departed from the premise that the cluster would be able to cover the additional costs of the work, and discussed in detail the features to be implemented in order to have the Portal ready for the launch.

Members of the Website Task Group also committed time to some of the pending tasks. One offered to create the world map. Another volunteered to again test the database's routines for the Expert Profile and Company Profile.

\footnotetext{
${ }^{20}$ Such as the search engine, the content management modules for products, and the "private" Project Sheet, as well as the development of web pages for "International Visitors" and "News and Events."
} 
I wrote a memo inviting cluster members to submit websites for the "Interesting Links" page. Lack of time and commitment hindered attempts to collect content for this page. Other pressing matters were at hand and "Interesting Links" was not a critical page. Moreover, the cluster Facilitator informed me that after a general invitation like this it was common to follow up on a one-on-one basis. He also promised to provide some links later on.

I met with the developer on 14 April 2005 to show him a draft of a new Feedback Document based on the comments from the Website Task Group meeting. We both went through the draft and negotiated which of the listed tasks were within the current contract and which would require an additional contract.

On 18 April 2005 I distributed the revised Feedback Document. I also circulated the developer's quote for the additional job. Unfortunately the cluster Co-Chairs and the cluster Facilitator were on business abroad, and thus any decision about which jobs would be done for the Portal would have to wait. Co-Chair Two requested a meeting with the developer. He was keen to organise the additional contract and finalise how to move the project forward as soon as he returned.

During our meeting with the developer on 28 April 2005 we identified the four main jobs for the new contract. The first two jobs in the quote (i.e. rearrangement of the template, and adding the world map and hotlinks) needed to be done to have the web site working by early May. The last two items (i.e. create administration systems for the "Interesting Links" and "News and Events" pages) could be developed in future versions of the Portal.

Co-Chair Two provided the extra funding for the developer to start work on the first two points of the second quote. A decision had been made without going through the process of e-mailing around. This Co-Chair had decided to take a more proactive role.

On 10 May 2005 the Website Task Group member who volunteered to work on the world map e-mailed the final version of the world map.

The next cluster meeting took place on 25 May 2005. The developer was unable to deliver an updated prototype of the Portal on time to show the cluster. Nevertheless, CoChair Two and I briefed the cluster on our progress. Appreciation of the additional funding contribution made by the Co-Chair's organisation to ensure the completion of the Portal was recorded in the Minutes. 
Co-Chair Two expressed his belief that the website was going to be a useful tool for members when they are abroad. He gave the example of his recent trip overseas, where he had to contact his colleague in New Zealand several times to get information about experts. Co-Chair Two was of the view that he could have avoided this inconvenience if the Expert Profiles and Project Sheets were available from the website at "the tips of his fingers."

Around this time, a cluster member who had been inactive contacted the cluster Facilitator. He wanted to update his CV and photograph. As the cluster member was unaware of the upcoming "new" Portal, he also offered to look at the content of the old website. I sent an e-mail to this cluster member and asked him if he could review content of the prototype instead. He agreed and promised to respond with comments.

On 28 June 2005 the developer e-mailed me to say that he had completed the corrections to the Expert Profile routines and therefore this part was ready for testing. I requested the Website Task Group member who had offered to test the Portal to proceed with some data entry. As with the first tester, I asked her to keep a record of errors she encountered, as well as noting suggestions on how to improve the system.

The second round of testing showed improvements in the system. The second tester's feedback focused on how to make the routines and online forms to perform as she expected them to. Like the first tester, she believed it would be valuable to develop "smart" templates that show how the information is going to be displayed and that provide sufficient details about the type, format, and style of the information needed in the system.

On 5 July 2005 I met with two Website Task Group members to discuss the NHNZ website. I raised the fact that it would be good to have a Task Group meeting to define guidelines for the cluster about what data to enter into the system, for example, how far back would cluster members be allowed to go to enter their experience or track record.

The next day I met the cluster Facilitator. I told him about my meeting with the other Website Task Group members and that we believed it would be a good idea to organise a Website Task Group meeting to address the issues raised. The Facilitator, however, considered it difficult to assemble the team as members were focused on bid projects (e.g. Aceh and the 2004 Boxing Day tsunami). He also expressed concerns about making each cluster member responsible for entering their own Expert Profiles and Company Profiles for the first time, because completion of this could not be guaranteed. 
Instead, he was inclined to call for volunteers to enter this information on cluster members' behalf and then ask the members to review it.

The Facilitator called Co-Chair One to propose this option. The Co-Chair agreed to provide staff to enter Expert Profiles. The Facilitator then spoke with the Liaison person from Positively Wellington Business (PWB) and asked if she could also help. She volunteered some time to do so. Despite this positive outcome, I was concerned about not having a Website Task Group meeting to examine some rules or guidelines on how to use the Portal's system and the new genres.

Days later I contacted the cluster Facilitator to initiate work on the "News \& Events" page. This section - which was not featured in the old website - would provide a space in the Portal for the cluster and individual members to post ready-made news and events stories about themselves (e.g. clips from various conference papers/news, initiatives they have put out).

On 15 July 2005 the cluster member who agreed to review the Portal's content contacted me with the awaited feedback. He provided in-depth analysis of the content for the main pages. I incorporated his suggestions regarding wording and layout, but those observations about the way the information had been presented would need some kind of consensus from a group - not necessarily the Website Task Group ${ }^{21}$.

During NHNZ's Annual General Meeting, I presented the latest prototype. I demonstrated the improved look of the website (e.g. the altered font size, the Home Page, the included hot links and world map). I also entered into the restricted area of the Portal where members could soon enter/edit information about their companies and staff. I then informed the meeting that a cluster member and PWB had volunteered staff to enter the Expert Profiles, so members would need only to log in and make the pertinent corrections to the information after their data has been entered.

Finally, I suggested creating a Content Task Group to review the Portal's content to release the current Website Task Group, which had worked on the project for a long time. Apart from the two Co-Chairs and the Facilitator, who felt they should remain involved, three new cluster members and a fourth person from the old Website Task Group volunteered to be part of the new Task Group.

\footnotetext{
${ }^{21}$ A few days later I talked with this cluster member over the phone and asked him if he would be happy to help out in a new Task Group to look at the Portal's content.
} 


\subsubsection{Discussion Fourth Iteration}

In the fourth iteration some Website Task Group members phased out their participation. Fortunately at the same time others started to get more involved, so the level of commitment by the team remained relatively constant:

And then, [another Task Group member] started to pick up somewhere in the time that I started to drop-off. [...] He does website design himself so he was coming with a whole bunch of ideas... (Interview Task Group member, 03 November 2005).

By now the Portal development had taken over a year and there were signs of change in the dynamics of the Website Task Group. The sense of urgency to complete the Portal was starting to build, which lead to the use of alternative means to make decisions rather than calling for the whole team to do so.

Major decisions on how to proceed with the Portal's development began to be made by key players with authority. For instance, one of the cluster Co-Chairs negotiated the second contract with the web developer and found funds to cover the additional work. Another example in this iteration was the intervention by the cluster Facilitator, who directly approached two organisations and asked for their assistance to enter CVs into the system. Thanks to his action the main problems were addressed without calling for a Website Task Group meeting.

The decisions carried out by different people in the Task Group are consistent with the concept of distributed leadership proposed Zhang and Faerman (2007). For these authors the leadership role is fulfilled by several individuals during the development and implementation stages of an information system, instead of having all the responsibility vested in one person as proposed by traditional leadership models.

There were two instances in this fourth iteration where we involved the rest of the cluster community in the Portal's development. We requested their feedback for the descriptions of each hazard and we asked for contributions to the "Interesting Links" page. Unfortunately there was only one response to the first request and none for the second. At this stage, we did not worry about the lack of response by the members as there were other urgent jobs to be completed before the Portal was fully operational. However this signalled a potential problem - identified by Kappelman et al. (2006) as lack of stakeholder commitment or participation - for the next iteration, when we would need their participation to populate the Portal. 
The second round of usability testing of the Portal's database during the fourth iteration confirmed the need to create the "smart" template for the Expert Profile, which should have detailed descriptions on the type of information it required. It was expected that in the following iterations members would contribute their experts' details; therefore this template should be ready by then.

We continued to see instances of members in the Website Task Group putting forward their knowledge and expertise towards building the Portal, such as in the case of developing the world map.

The content for the Portal was another way of reusing material already available. Examples of this material were: old brochures, the marketing strategic plan, and a cluster profile. The material required some rewording to fit the needs of the Portal, but it was a good starting point.

The "News and Events" page started to be developed in this iteration. The primary goal for this page was to inform the target market and other cluster members about NHNZ activities, events, and relevant news. Nevertheless, this section could also play a second important role in terms of the cluster's knowledge-base. "News and Events" could give the cluster an organised record of its activities and achievements, thus improving the cluster's organisational memory.

\subsection{Fifth Iteration: Developing the final version of the NHNZ portal}

The fifth and final iteration of the Development Stage began on 29 July 2005, shortly after the NHNZ Annual General Meeting, and concluded on November 2005 with the Portal's launch at Te Papa National Museum ${ }^{22}$. This iteration comprised of preparing content for the portal, entering the details for the Expert Profiles and Company Profiles, developing the Project Sheet functionality, and entering examples of Project Sheets.

\subsubsection{What happened in this Fifth Iteration}

With the help of the PWB Liaison I prepared two letters: Letter to the Primary Contact and Letter to the Standard User. These letters would be sent to members once their details had been entered into the system.

\footnotetext{
${ }^{22}$ Te Papa is New Zealand's National Museum, located in Wellington.
} 
The Primary Contact is the person in the organisation assigned to be the first point of contact for issues related to the Portal. This person will have access to edit their Company Profile, the details of their colleagues' Expert Profiles, and their Project Sheets. The Letter to the Standard User was addressed to those experts who were going to be registered in the system by their organisations. Their letter introduced the Portal and asked them to visit the portal and review their information. Standard Users would have access to edit only their own Expert Profile.

On the website content front, one Content Task Group member submitted links to be included in the "Interesting Links" web page. Some of her suggestions were incorporated along with others provided by the cluster Facilitator.

I visited one of the volunteers working on the Expert Profiles to check on her progress and to collect impressions on how the forms were performing. She found the system easier to work with now than on her first occasion (she was the tester of the system back in March 2005). Although she was able to complete the online forms, she recommended the inclusion of further instructions on how to fill in some of the fields to avoid users entering errors which they could not delete. For instance, the way to enter new categories for the fields "Areas of Expertise" and "Qualifications" was not clearly explained in the form and thus the drop-down list was filled with incorrect or misspelled categories, which made the list long and confusing ${ }^{23}$.

During September 2005 the PWB Liaison - the other volunteer working on the Expert Profiles - noticed that not all the registered specialists had a one-page CV in the old website. She therefore e-mailed these members requesting their details for her to enter onto the new Expert Profiles. The PWB Liaison also contacted people in the organisations who would become the Primary Contact for the website and sent them the Letter to Primary Contact and the Letter to Standard User.

During the NHNZ cluster meeting on 15 September 2005 it was alerted that the cluster will be celebrating its fifth anniversary in November and therefore that could be the appropriate time to launch the new Portal. The PWB Liaison mentioned the disposition of her organisation to support such an event and associated promotional activities.

\footnotetext{
${ }^{23}$ This problem escalated when cluster members started to indiscriminately enter categories into these fields. It was not until January 2006 that the developer prepared the routines and forms for the portal administrator to delete entries that were misspelled, repeated, or incorrect. On 13 February 2006 I undertook an initial clean-up of these items.
} 
A Launch Task Group was created to organise this event. This Task Group comprised of this cluster Co-Chair and two other cluster members, along with support from the PWB Liaison and the cluster Facilitator. This Task Group was effective in securing New Zealand's Prime Minister, the Right Honourable Helen Clark, and Wellington's Mayor Kerry Prendergast to attend the event and officially launch the new website.

The other topic covered during the cluster meeting was the Portal's content. It was agreed that it would be useful to have a two-hour session to review the Portal's content by the newly formed Website Content Task Group. Finally Co-Chair One mentioned the urgency of having the Project Sheets completed before the official launch.

PWB agreed to fund the services of a small Wellington-based web design firm to edit and upload the Project Sheets into the new Portal. Having a sample of Project Sheets edited and uploaded was a pressing priority for the launch; consequently the content in other pages of the Portal remained unchanged ${ }^{24}$.

As a follow-up to this cluster meeting, I met with Co-Chair One on 21 September 2005 to discuss the Project Sheet genre. Back in October 2004 the Website Task Group agreed to some fields for Project Sheet, but hold-ups in the development of the Expert Profile delayed work on this second component of the database.

I brought to the meeting the document with the table about the fields for the Project Sheet. I also brought a printout of the project summaries that this Co-Chair circulated in the first iteration, with my notes on the fields as agreed by the Website Task Group in October 2004. We reviewed these documents and made minor changes to the template.

Why did I rely solely on this Co-Chair to check the fields for the Project Sheet? Firstly, he was the Website Task Group member who had been pushing this component of the Portal. Secondly, he was one of the NHNZ Co-Chairs and a champion for this project. Thirdly, this meeting with him was to confirm what the Website Task Group had agreed back in October 2004. Finally, the other members of the already-dissolved Website Task Group had distanced themselves from the project ${ }^{25}$.

\footnotetext{
${ }^{24}$ The idea of having a copywriter review and create content for the NHNZ Portal was left on hold until April 2007, and is thus outside the timeframe of this study.

${ }^{25}$ The cluster Facilitator suffered funding cuts for his services this year; he stayed involved on the core cluster activities and relied on my assistance to drive the Portal's development. Co-Chair Two had been away, but I had been communicating with his work colleagues who were also members of the Website Task Group team. The remaining two Website Task Group members expressed some time ago that they were going to step down from active involvement.
} 
On 26 September 2005 the PWB Liaison sent a memo to the cluster announcing that the Natural Hazards New Zealand cluster function would take place on 10 November at Te Papa National Museum from 5:30 pm onwards. She estimated 120 people would attend. According to her memo, the purpose of the function was:

- To celebrate NHNZ's 5th birthday.

- To thank those who have assisted - i.e. stakeholders, embassies and other agencies.

- To launch the new website, which will enable prospective customers to search for specific capability within the cluster.

- To showcase a successful Wellington cluster.

- To promote the cluster to people who may be able to help enter new markets, or deepen existing markets. (E-mail PWB Liaison, 26 September 2005).

Shortly after this announcement I met with the web developer. I mentioned to the developer that an official date for website launch had been set. We discussed the next deliverable of the project, which was the Project Sheet. The web developer believed he could have this component completed before the launch date, as he was planning to travel overseas in late October.

On 29 September 2005 I sent a memo informing the cluster of progress. By then threequarters of the Expert Profiles had been entered into the system. I encouraged members to visit the website and review/edit the information that had been entered for them and their organisation. The PWB Liaison replied with the following comment:

Whilst most people have been sent the letter inviting them to check the details of their CV on the site, I doubt many have followed this up ... and it may take quite a few phone calls to make this happen. (E-mail PWB Liaison, 30 September 2005).

That was precisely what occurred throughout the month of October. The PWB Liaison and I spent many hours reviewing the Company Profiles and their Expert Profiles. We contacted individual members via telephone or e-mail to remind them that the quality and accuracy of the information in the new NHNZ Portal would depend on them.

By early October more cluster members started to contact us confirming their primary contact, requesting access to the website, or asking for technical help in using the database. The exchange of e-mails between the PWB Liaison, cluster members, and myself regarding the website intensified as the launch day drew near. On the eve of the launch the cluster Facilitator contacted the lagging companies to collect their 
information for the Company Profile, but still a small number did not submit their content on time.

The Content Task Group agreed to meet on 14 October 2005. The meeting was attended by the Facilitator, the two cluster Co-Chairs, three cluster members, and myself. The meeting began with good attendance; however people left at various times to attend other businesses. On discovering that several members were going to leave early I looked at the most pressing issues first - those where consensus on how to proceed was needed. For example, I showed the draft for the public Project Sheet genre that CoChair One and I had been working on. The group approved it as the final version.

On 14 October 2005 the web developer notified that he was not confident that the Project Sheet functionality would be working properly by the time of the launch. He was taking leave at the end of October and although the system would be developed before his departure he would not have time to test it thoroughly. The web developer was concerned about introducing an unstable element to a mostly stable website. He left it to the cluster to decide whether to include the Project Sheet on the system for the launch. After consulting with the Co-Chairs and the cluster Facilitator, it was agreed that the Project Sheet component should go live as planned. That was a risk the cluster was willing to take.

On 3 November 2005 I met with the cluster Facilitator to review the status of the Portal. Attention was paid to the Company Profiles and the Contact Us, Hazards, and Services pages. We were in "launch mode," that is, not worrying about the content for all the pages in the site, but making sure that the key pages had some content.

On 7 November 2005 the company contracted to edit and upload the Project Sheets into the system sent a memo to cluster members with a Project Sheet template. They gave general instructions on how to fill the template, to whom it should be submitted, and by what date. This template was in Word and was a simplified version of the online forms that feed the Project Sheet ${ }^{26}$. It had clear instructions and was easy for users to fill in.

Prior to his departure, the web developer introduced me to another developer who would address technical questions in his absence. Interactions with this second developer demonstrated that he was committed to the project despite having his own

\footnotetext{
${ }^{26}$ At a later date I produced another template version for Project Sheet, which combined the layout of Project Sheet and the instructions found in the template by the web design contractor. The contractor showed me a better way of asking users how to fill-in the template.
} 
workload. He was quick to respond to my requests for help in solving programming glitches (especially on the Project Sheet functionality which was not fully tested), uploading web pages, and fixing a major system failure two days before the launch when the website was migrated from its old host. In certain instances the developer provided temporary patches, leaving the proper solution to be implemented by the lead developer upon his return.

On the day of the launch the cluster Facilitator and the two cluster Co-Chairs were at the entrance to the Te Papa Museum greeting guests and waiting for the Prime Minister to arrive. The event facility upstairs was already crowded with people. Figure 16 is a screenshot of the Home Page of the new NHNZ Portal.

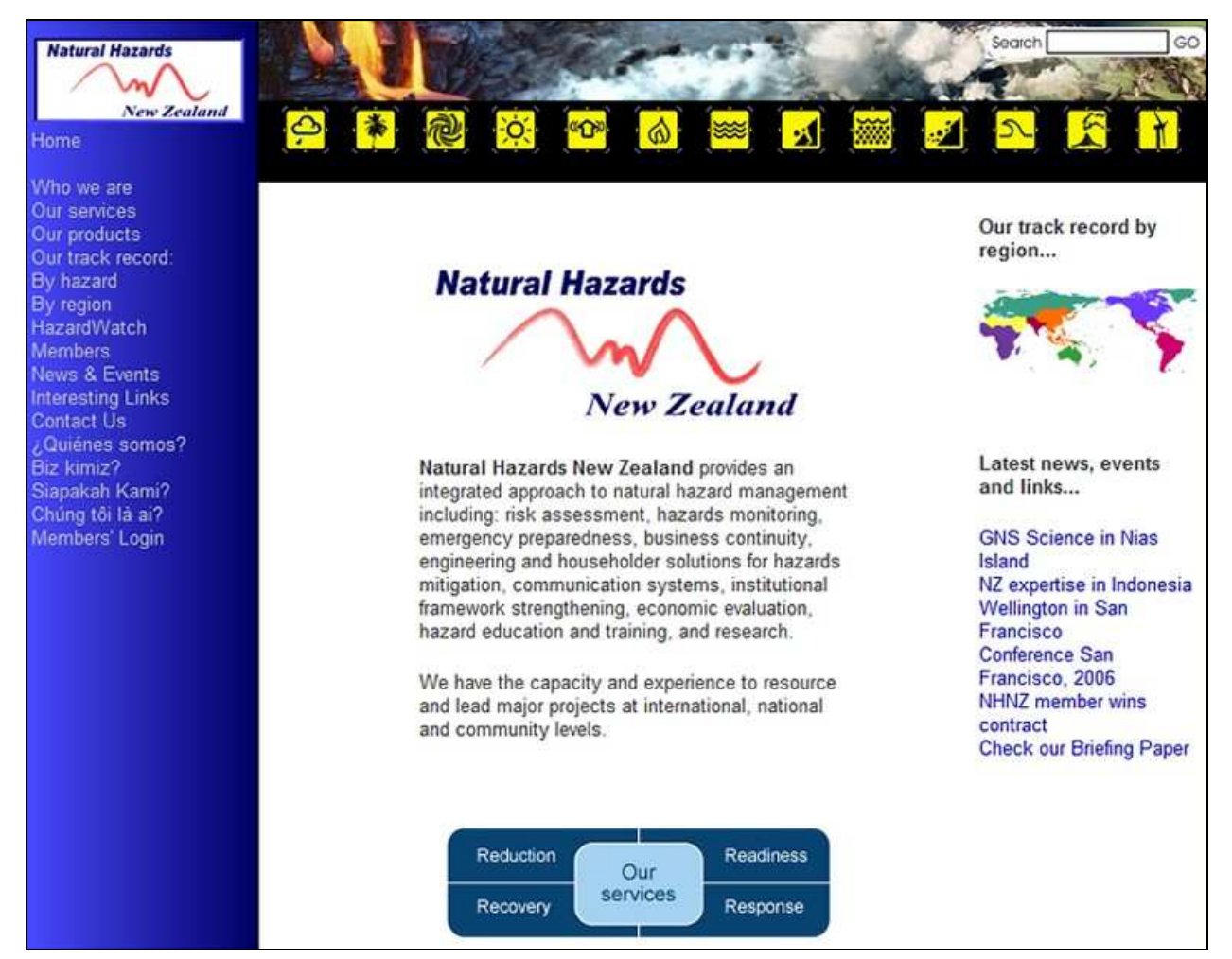

Figure 16. The new NHNZ Portal's Homepage (source: www.naturalhazards.co.nz).

After the launch, the Co-Chairs, the PWB Liaison, one Content Task Group member, and I went out for dinner. There was an atmosphere of celebration amongst us. Both CoChairs were pleased with the success of the website launch and with the opportunity to talk to the Prime Minister about the cluster. It was interesting to witness their conversations and their ideas about what to do next with the Portal to make the most of this renewed interest in the cluster. Here we could see the intention to gear up to another action cycle. 


\subsubsection{Discussion Fifth Iteration}

The cluster decided to launch the Portal in its fifth anniversary celebrations. The Portal launch became an important event for the cluster when Prime Minister Helen Clark and Mayor Kerry Prendergast confirmed they would be guest speakers. Defining a date for the Portal's official launch motivated cluster stakeholders to complete the Portal.

In the final iteration members were involved in the development of the Portal at various levels. Some volunteered to be part of either the Website Launch Task Group or the Content Task Group and thus were directly involved in the process. Many submitted examples of projects to showcase in the Portal according to the Project Sheet template and reviewed their online Expert Profiles.

Some members were proactive and accessed the private area of the Portal to enter the information for their Company Profile genre and Project Sheets rather than asking us to do it on their behalf. Having cluster members interacting with the system to create their specific instances of the genres, gave us the opportunity to test how well these genres were conceptualised by a larger group of users. Based on their feedback, the genres were improved to ensure they deliver what it was expected.

In this iteration, little time and resources were allocated to developing detailed documentation on how to interact with the system, except for the Primary Contact and Standard User letters. There was a reliance on the system being self-explanatory, and thus an effort was made in writing clear instructions in the online data entry forms based on the results from the usability tests. However, feedback from members indicated that the instructions in some of the forms were insufficient. Garceau (1990) pointed out that, without proper documentation, users are unable to employ the system to its full potential, and over time details on how to do things with the system are lost. 


\section{Chapter 9: The Portal Evaluation Stage}

As discussed in the Methodology Chapter of this study, action research gives the participants freedom to choose which type of evaluation they want to use. This is understandable, given that it depends on the nature of the problem, the context, and the needs of the action research team.

The form of evaluation carried out in this study followed what Owen (2006) describes as "interactive evaluation." Here, those who participated in the intervention stage are closely involved in assessing the outcome of the actions taken. There is an expectation that findings resulting from the evaluation will enrich the organisational knowledgebase and improve future interventions.

This study considered three types of evaluations: the first where portal development was assessed following a prototyping approach and user-centred design (systems design evaluation); the second where the system was compared against the initial specifications and contract with the website developer (project evaluation); and the third where the project research team reflected on what happened during the development process (participant evaluation). It is in this last type of evaluation that the team discussed how the ICT intervention improved knowledge creation and transfer within the cluster, as well as suggested ways to improve the system.

The reader is reminded that, due to the scope of this research, I did not develop an assessment tool to evaluate the effectiveness of the new digital genres. Instead, I relied on anecdotal accounts by the participants to provide an indication of the usefulness of these knowledge transfer mechanisms. Developing and applying an evaluation tool to assess if the digital genres remain relevant for the cluster can be considered in a future action research cycle.

\subsection{Systems design evaluation}

Since the project was ICT in nature, it was logical to follow a system design evaluation, where the routines, data entry, screens, and overall functionality of the system are checked to ensure they work according to expectations. Due to the prototype approach used by the web developer to create the new NHNZ Portal, evaluation was an ongoing activity during the five iterations of the Development Stage (Baskerville \& Stage, 1996). Usually each iteration concluded with the Website Task Group (or some of its 
members) meeting with the developer to review the prototype. A feedback document was prepared to record the required changes.

Prototyping allowed the Website Task Group to identify the gaps in the portal and to visualise the final system, which in turn led to them considering additional features that were omitted in the initial specifications. For instance, after reviewing the first prototype, the Website Task Group requested a form by which users provide their email addresses to the cluster members rather than cluster members having their contact details displayed on the website; this was designed to avoid unwanted e-mail harvesting for spamming purposes.

Prompted by the prototype, the Website Task Group was also able to reach consensus about what changes should be made. For example, assessing the prototype at the beginning of the second iteration helped the Website Task Group realise that some fields for Expert Profile and the Project Sheet were not suitable. Sadly, in this instance, programming work done by the web developer had to be rewritten.

In addition to the review by the Website Task Group at the end of each iteration, I logged in to the Portal and test it out as soon as a component or function was released by the web developer by carrying out the tasks that the particular component was expected to perform. When something was not working properly, did not look good, or was confusing, I notified the web developer via e-mail and kept a record of the error and included it in the next feedback document:

Hi [web developer], I have been playing with the NHNZ web prototype and spotted some things that hopefully are not too difficult to sort out... (E-mail Researcher, 17 December 2004).

Up till the third iteration, the web developer and I were the only ones testing parts of the portal and its database. In the third iteration and afterwards, the prototype was sufficiently complete to be trialled by a small number of users to ensure the system followed user-centred design.

User-centred design aims to create consistent and better "usable" applications or artefacts by focusing on how the user interacts with it (Manzari \& Trinidad-Christensen, 2006; Prasse, 1991). It involves the user at early stages of development to ensure user needs and ease-of use expectations are incorporated into the design. User-centred design has been applied in website and e-commerce developments for hospitals (Gallant, Irrizarry \& Kreps, 2007), corporations (Agarwal \& Venkatesh, 2002), and university libraries (Manzari \& Trinidad-Christensen, 2006; George, 2005). 
Usability testing is an empirical method for user-centred design. Prasse (1991) defined usability testing as "the process of obtaining information on how easy is to learn and use a product from the expected users of the product" (p.10). The purpose of this information-gathering process is to improve the design of the product - which in our case is a website portal - and thus ensure the user's experience of it is satisfactory.

Usability testing of the NHNZ Portal was performed primarily by two participants from two different companies belonging to the cluster at two different points in time. The first round of testing took place in early March 2005, while the second occurred in July 2005. In both cases I organised instructions for the testers and provided them with training on how to use the system. Once the testers completed the tasks, I interviewed them about their experience with the system. I collected their observations, which became part of the feedback document.

The usability testing in both instances allowed us to identify errors in the routines and forms that would have caused frustration to cluster members if we had omitted this part of the development process. Both testers identified areas of the system that could be improved. In their roles as users, they provided practical suggestions on how to address these shortfalls. Finally both testers pointed out the need to develop "smart" templates that not only show how the information is to be displayed but give sufficient details about the type, format, and style of the information to be entered.

\subsection{Project evaluation}

Following the traditional project management perspective, project evaluations are events that take place to assess whether the project is progressing within its scope and according to the specified cost, schedule, and technical performance. Cleland and Ireland (2006) indicate that project evaluations can be either a routine exercise in a project, or carried out only when there is a perceived problem.

In this study, a simple tool was developed to assess the progress of the Portal development against the initial requirements. This tool was a table that compared each of the items from the Website Specification document, the actual contract with the developer, and what had been delivered. In essence, this table was a checklist of which features had been completed and which ones were pending at a particular point in time. An example of the assessment tool can be found in Appendix B. 
I first completed the table based on what I could see in the prototype and then submitted it to the Website Task Group (or only to the cluster Co-Chairs) for their consideration and input. A meeting with the web developer followed to collect his views on the findings of the assessment.

This evaluation tool was used three times during the life of the project: the first time as support material for the meeting on 1 October 2004 when assessing an early version of the prototype; the second time on 13 January 2005, as a reminder of where the project was at after a few months of low commitment by the Website Task Group; and finally on 19 December 2005, after a request from the Co-Chairs, who wanted to know what had been delivered for the purpose of signing-off on the project.

There was one instance during the fourth iteration where I assessed the prototype against the initial requirements, given that it had been a year since the Website Task Group first met to discuss the development of the Portal. We discover that some functionality expected for the Portal was not included in the signed contract. The discrepancy was noticed by the assessment tool the first time it was used; however neither the Website Task Group nor I noted it at the time as we were focused on defining the database and the genres to include in the Portal. The omissions were notified to the Task Group and corrective measures followed. An additional contract had to be made with the developer.

Kappelman et al. (2006) indicated that weak commitment by the team in an IT project may cause delays and risk going over budget. In our case, we lost sight of the Portal's initial development requirements for a time as all members of the Task Group were working on the project on a voluntary basis. All of us had other primary responsibilities (i.e. paid work and studies) and consequently our attention to the Portal was intermittent. Resuming the project from where it had been left entailed additional time spent recalling important points about the Portal's purpose and recalibrating our attention on addressing the web developer's questions.

\subsection{Participant evaluation}

Ad hoc evaluations were made by cluster members after they were invited to populate their respective sections of the new NHNZ portal. These members e-mailed their comments about their experience with the system and provided suggestions on how to improve the portal in terms of ease of use, content, and functionality: 
It took me some time to work out how to put my own entries into the drop-down menus via "other" and "continue" - you should arrange to install a helpful note to avoid time-wasting by others. (E-mail cluster member, 03 November 2005).

I was trying to find out some more information about cost of membership to the cluster on the website, but couldn't seem to find more information. [...] Is this information that should be included on the site in future for prospective members? (E-mail cluster member, 16 November 2005).

Another suggestion is to make provision for video clips such as the TV One National News Item about the Te Papa tsunami design featured on our [company website] - this often presents something in a way which does not get across in a sterile written paragraph. (E-mail cluster member, 22 November 2005).

The action research inquiry, however, expects a more careful and structured evaluation process after an intervention than the feedback provided by cluster members. The reason for this is to gain understanding about what happened and why, as well as to have a space where participants and the researcher can reflect on the intervention and suggest ideas that could be implemented in future action research cycles (Baskerville, 1999; Davison et al. 2004).

The third type of evaluation in this study was introspective in nature. It subscribed to the principle of having a space where the participants recall the experience and make inferences, generalisations, and their own assessments about the intervention (Wong, Loke, Wong, Tse, Kan, and Kember, 1997). It provided opportunity for the team to critically question whether the intervention was successful, and to identify a framework for future action research (Baskerville, 1999).

This approach is consistent with recent project management views that project success cannot be evaluated just in terms of project implementation by achieving the intended outcomes of specification, time, and budget (Andersen, Birchall, Jessen \& Money, 2006; Ojiako, Johansen \& Greenwood, 2008; Jugdev \& Müller, 2005). It has been argued that a broader perspective where long term economic, social, and environmental wellbeing of the project stakeholders should also be considered.

Members from both the Website and the Content Task Groups reflected on their experience of developing the Portal and how it had improved knowledge creation and transfer within the cluster community. They also contributed with suggestions for features and templates to incorporate in the Portal and how the development process could be improved for the next intervention. 
Their reflections were collected through interviews: three Task Group members were interviewed on the eve of the NHNZ portal launch or shortly thereafter, and four others were interviewed seven months later. In addition, the reflective comments made by the two prototype testers during their interviews were taken into consideration.

The following sub-sections are the main themes that emerged from the comments of those interviewed. In-depth analysis of these themes would take place in the specifying learning stage, which is covered in Part 3 of this study.

\subsubsection{Subscribing to the Task Group approach}

NHNZ culture involves delegating specific activities to Task Groups. Once a cluster has decided that an initiative is worth pursuing, a Task Group is formed to follow it up and report back on it during cluster meetings. Members of the Website Task Group acknowledged the practicality of having a team rather than the whole cluster working on the design of the new Portal and therefore volunteered their time to do so.

Several participants agreed that the Task Group approach was effective in defining the Portal's Specifications Document at the Diagnosis Stage. However they felt that having a Task Group overlooking the development of the project was inappropriate. In the Development Stage there were too many individuals whose inputs needed to be considered and usually consensus was reached only during face-to-face meetings; something that was difficult to organise due to the individuals' work commitments, business travel, and responsibilities with their own companies. Consequently the Portal took longer to be completed.

It was pointed out that ongoing project management should have been assigned to a smaller number of people than those involved in the Website and Content Task Groups, something to be considered in future portal developments:

I don't think you need the committee any longer, really. I think with someone like yourself driving it and then just shooting the question out when you need to - say the Co-Chairs and the Facilitator - that's about all we need now. (Interview Task Group member, 14 December 2005).

\subsubsection{Learning about system design}

Members of the Website Task Group learnt about system design from the experience of preparing the Specifications Document and then going through the Portal development process: 
I learnt lots, tons! It just made us think much more how do we want to structure things up and what we really wanted from things, and do it in a thorough way. And also to understand, especially when you are thinking of databases and whatever, what are the different approaches. [...] And how the users would use it! (Interview Task Group member, 06 December 2005).

Although the Website Task Group had a general idea what it wanted the system to do, it was not expecting to make detailed decisions about components of the Portal. Consequently, it took the team some time to understand it, especially because most of them were not familiar with database and website technology.

One participant justified the time taken in defining the Portal given its complex nature where more aspects needed to be taken into account:

Well, I think you need to be more thorough in a situation like this because you [sic] got so many more people not just viewing it but being able to edit it. And that's when you get into a dangerous situation if it is not working correctly. [...] So, unlike a normal website that is just read only and is information for people. This is a little bit different. [...] It may take a little bit longer but the end result will be a lot better. (Interview Tester One, 08 March 2005).

Team members mentioned that incorporating a database, using templates, and working with a prototype were at times challenging concepts to grasp. This raised the question of whether it would have been better to put the Portal development on hold until the Website Task Group was ready to discuss the specific principles of the system. People underestimate the relevance of having a good understanding of an ICT system in its early stages of development.

Upon reflection, a member of the Task Group said he would have followed a different approach to speed up the team's learning curve based on his current knowledge of the situation:

If I were approaching all this again [developing the Portal] with a budget, I would say, even to bring in someone like that [who understands the technology] at an early stage and say, "Look, it would help to figure out what you actually want." It is worth pulling someone like that, paying two, three days work, just to help to inform us up and make the strategic sense right at the beginning. (Interview Task Group member, 06 December 2005).

\subsubsection{The cluster knowledge-base}

Participants are pleased that the new Portal provides a space for storing experts' profiles, project summaries, contact details, and general information about company 
members. For instance, a participant mentioned that some companies do not regularly attend the cluster meetings and therefore there is no opportunity to learn from them about their areas of expertise and their staff. The Company and Expert Profiles now available online have been a way of overcoming this shortfall with information about these companies, thus increasing awareness within the cluster of its own capability pool:

It's good reading about other organisations. I have taken the time to read through because not all businesses turn up at the cluster meetings. In reality you don't get the in-depth time you might need or I want to find exactly what they are on about [during cluster meetings]. So I found it very useful like that. (Interview Task Group member, 04 July 2006).

Along these lines, another participant commented that the Portal had the potential of helping members to identify complementary partners for bidding on projects.

An aspect about the intervention that delighted one of the participants was the fact that a number of Project Sheets were finally produced during this project; resources that could be used in their expressions of interest or as part of marketing material.

Several participants mentioned the convenience of being able to make changes to their information as needed and when they were ready to do so without having to go to a third party (i.e. the web administrator or cluster Facilitator). Such functionality in the Portal would allow regular updates of parts of the cluster's knowledge-base.

\subsubsection{The use of the resources in the Portal for project bidding}

Since the Portal went live, there was limited use of its resources for project bidding in the subsequent months by those interviewed. One participant had used the Expert Profile of one of her colleagues to submit as part of an EOI. This participant also used the Expert Profile of another cluster member who was collaborating with them in a particular project to prepare a press release. Even though this second instance was not directly related to project bidding, it showed that the Portal was providing the virtual space to place information for reuse that cluster members have agreed to share.

Another participant mentioned how easy it was for him to prepare hand-out material about the cluster and its capabilities for a delegation from Turkey, where several cluster members have recently won a project. This material complemented his relationshipbuilding endeavours, an activity critical in the proactive project bidding approach.

Many participants reflected on the various levels of commitment showed by cluster members with regard to this project (and any other cluster initiatives put forward for 
that matter). Although it would be nice to have every member on board, they are realistic that this would depend on each member's perception of what they would gain from involvement.

\subsubsection{What can be done to improve the NHNZ Portal}

Completing the Portal for its official launch on 10 November 2005 was a very intensive and demanding exercise for the Task Groups and for the rest of the cluster. Therefore it was not a surprise to hear several participants commenting that they wanted to leave the Portal as it is for a while - apart from keeping the content current.

Nevertheless, there were expressions of commitment by several of the interviewed participants in developing the Portal further:

The nature of working in technology and the nature of working in the field we are, it would always have a kind of ongoing development. That's the nature of it. In particular, you know there are so many businesses in the cluster, I wouldn't imagine [further development] not happening. (Interview Task Group member, 04 July 2006).

As another participant pointed out, the Portal is always going to be a "work in progress." On this premise, I asked the Task Group members to suggest refinements or features they would like to add to the Portal in future versions.

Most of the interviewees felt that this intervention focused on building the framework of the portal, the database, its routines, and the new genres. Therefore for them the next development cycle should go over the Portal's content to ensure the descriptions of services and capabilities reflect what the cluster members do, reviewing the wording and meta-tags used to guarantee the Portal will get high rankings in search engine results, and increasing the pool of Experts Profiles and Project Sheets.

A different - but not conflicting - suggestion made by another team member argued for the next cycle of intervention to review the website's presentation:

The design, just the design! ...does it look like a real place I want to be or does it look a little bit pedestrian? It is not saying anything against the information that it is in there, it is just branding. [...] How it would look to this famous World Bank task manager? Because at this stage... I would like it another notch or two up before I really go shouting it from the rooftops. I don't think it is quite rooftop material yet. (Interview Task Group member, 14 December 2005).

In addition, this same participant proposed that future versions of the Portal link to other websites that organisations like the United Nations, the US Geological Survey, Oxfam, and World Bank set up after a major natural disaster - such as the 2004 tsunami in Asia 
or the 2005 earthquake in Pakistan. These sites post information about the natural disaster as well as their response and recovery work in the affected regions, information which cluster members may find relevant for their bidding efforts. Cluster members could also contribute with suggestions of links to other relevant organisations. According to this participant, this could be a useful feature to include in the NHNZ Portal to facilitate knowledge sharing within the cluster:

I think that would be very useful to get on the website. [...] We don't have to simulate all the news and put them in the website. All we need to do is get the URL and just a quick one-liner about what that's about. In that way as members we just quickly get on to it. I'd find that very useful because even around here [in my company] various individuals know which website to go and look up, but they don't share it. (Interview Task Group member, 14 December 2005).

By becoming the port of exit to other resources available in the Internet, the website would behave more like a true Portal.

\subsubsection{The challenges for the new Portal}

Finally, participants reflected on the challenges ahead for the Portal. Their main concern was keeping the Portal and its content current. One participant suggested creating the role of Content Administrator to remind cluster members to update their parts of the Portal at various points in time. Participants were aware that the new Portal would require a more active involvement from the Portal Administrator than the old NHNZ website required. However it was not yet clear how much support would be needed.

The second challenge identified was carrying out the improvements to the Portal, in other words, to incorporate those features identified in the previous sub-section. Other pressing matters directly linked to winning bids would take priority over the limited time and resources available to the cluster:

And the reality is you always run that fine line between responding to a key thing that is happening now, you have to have a proposal now, versus taking the time to build the databases and to build comprehensive information systems when there is no immediate call on any particular aspect of that. (Interview Task Group member, 14 December 2005).

The cluster is realistic about its limitations. It has been creative in finding ways to overcome these shortfalls. Setting up small stages that are easy to complete and again relying on the goodwill of members to donate their time and expertise has been their strategy. 


\section{PART 3:}

\section{SPECIFYING LEARNING STAGE}

Part 3 outlines the findings of this research enquiry, which I am putting forward as my contributions to knowledge in the IS domain. I consider Part 3 to be the Specifying Learning Stage of this action research intervention.

Chapter 10 provides an answer to the first research question and suggests options for ICT to improve clusters' knowledge transfer during project bidding.

Chapter 11 and Chapter 12 address the second research question. In Chapter 11 a process of digitising genres is proposed; this model is the result of working with the cluster in one of the options where ICT assisted with knowledge transfer in project bidding. Chapter 12 provides an overall picture, based on the anecdotal evidence and the reflections of the participants on how ICT has assisted the cluster in creating a space for managing its knowledge-base.

Chapter 13 details supplementary findings of my study. While carrying out the research intervention, I was able to observe seven factors that influence how the clusters increase their knowledge-bases. This Chapter discusses these seven factors. 


\section{Chapter 10: The Project Bidding System}

The Diagnosis Stage aimed at understanding the project bidding process of EENZ and NHNZ - the subjects of this study. It identified two types of cluster bidding approach: reactive and proactive. Each of these approaches involves a series of steps or activities that need to be carried out in order to move the bid forward.

The collaborative interactions within each step were then mapped using the Genre Systems framework, which identified "Project Bidding" as the coordinated and communicative system of genres employed by the clusters to win an international contract. Please refer to Chapter 6 for an overview on how project bidding takes place in the clusters and how the Genre Systems framework was applied in this study.

The following two sections describe the reactive and proactive approaches used by EENZ and NHNZ. Then, the findings from mapping this process with the Genre Systems framework are put forward.

\subsection{The reactive project bidding approach}

Project bidding following a reactive approach is the result of responding to requests such as procurement notices and callings for expressions of interest (EOI), which have been put forward by external entities. In particular, the clusters pay attention to the official notices coming from the World Bank, the Asian Development Bank (ADB), and sometimes NZAID (New Zealand's operational arm for international aid).

The reactive approach involves competing with other companies and groups from all over the world, as these notices are openly advertised. In addition, it tends to be a long selection process (sometimes up to a year) that follows strict guidelines. Despite being a rigid and lengthy process, the projects in the reactive approach are considered "safe" in terms of receiving payment upon completion, due to the good reputation of the funding agencies.

Figure 17 depicts the activities that the clusters usually carry out during the reactive project bid approach. This diagram is the product of a series of interactions between the interviewees and the researcher. After each interview, the diagram was modified and then sent back to the interviewee for review and feedback. The interviewees found that the diagram illustrated what goes on during the bidding process. 


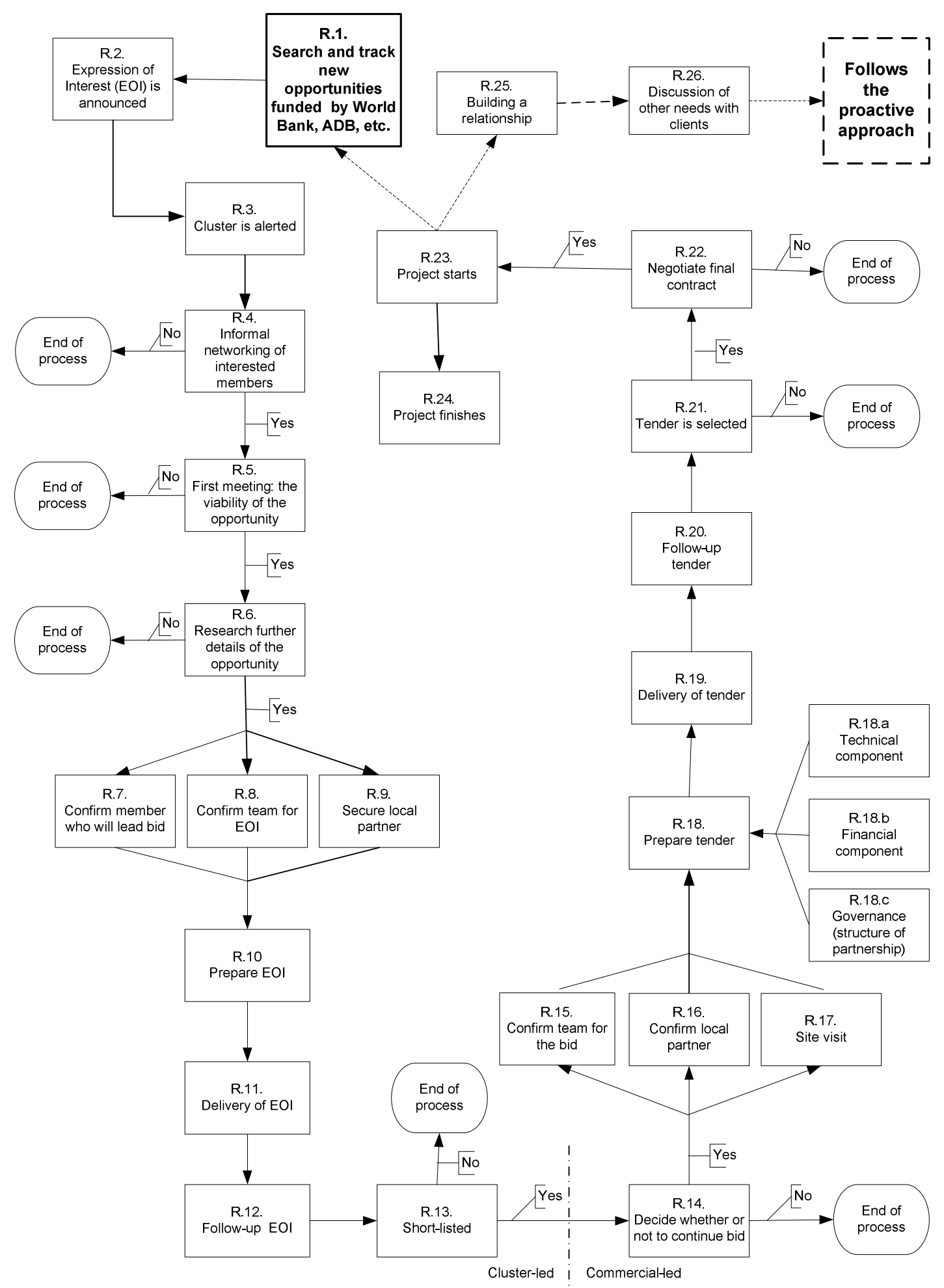

Figure 17. The reactive project bidding process followed by NHNZ and EENZ.

The activities are numbered starting from R. 1 to R. $26^{26}$ to facilitate easy reading of the diagram. A detailed description for each of the activities in the diagram can be found in

${ }^{26} \mathbf{R}$ stands for "reactive". A similar numbering code will be introduced for the proactive project bid diagram, but using a $\mathbf{P}$ for "proactive" instead. 
Appendix C. This numbering does not imply that each activity in a real situation follows a sequential order. In fact, many of these activities have been considered simultaneously, have been visited at a later stage, or are an ongoing exercise throughout the process - such as the case of building a relationship.

From the diagram we can see that the reactive approach is a complex process. There are two key selection stages - the short-listing of the EOI, and the selection of the tender before the winning team is allowed to negotiate the final contract with the clients. The diagram also shows that there is a constant assessment of the project's viability by those interested in pursuing the opportunity. Those involved in the bid make decisions at various points in regards to whether to continue or end the process.

EENZ and NHNZ clusters are actively involved in searching and tracking potential projects (box R.1). Within the clusters, there is an assembling of companies that are interested in particular countries or regions in the world. Usually they form Task Groups to watch for procurement announcements and calls for EOIs from the World Bank, the ADB and other funding institutions in those locations. Two examples are: the EENZ Turkey Istanbul Enterprise Network, and the NHNZ-EENZ Asia Earthquake \& Tsunami Recovery Task Group.

Calls for expressions of interest are not usually a surprise. An opportunity can be tracked for months - sometimes even years - before it materialises into an EOI. For example, the World Bank will first do appraisals of potential projects before committing any funding, and these reports are made available on their Website and their weekly newsletter. Tracking an opportunity involves planning, checking with local contacts, and even doing preliminary trips well in advance, so that when the opportunity arrives, the clusters are ready to tackle it.

One action the clusters have taken to track new opportunities is contracting New Zealand Trade and Enterprise (NZTE) services to monitor ADB and World Bank announcements. NZTE officials collect and send any information about projects, reports and events related to the clusters' areas of interest to Positively Wellington Business $(\mathrm{PWB})^{27}$. PWB will then circulate NZTE's information to the clusters via a distribution list. These monitoring services have proved valuable for the clusters, as most of the EOIs they have submitted have come from these sources.

\footnotetext{
${ }^{27}$ In this particular situation, PWB acts as the contact point for NZTE's officials.
} 
Nevertheless, the interviewees ${ }^{28}$ acknowledged that these announcements are not adequately filtered to the specific needs of the clusters. Large volumes of information tend to be passed on to cluster members, who have to sift through it before finding something relevant to their market or area of interest:

I spent a lot of time in the past opening up these attachments and scanning through the information and finding it haven't any link to our marketing plan, for instance. We have a marketing plan which says that we are going to target or be interested in certain regions and so on, for certain reasons. But my perception is that the information that arrives through my e-mails [...] to whet my appetite or to help my interest is not filtered by any means towards the marketing plan. (Interview cluster member, 15 October 2004).

The interviewees would like to see more value from the monitoring service than just passing messages on to the next link in the chain. They are also aware that the clusters may need to pay a premium for this additional service.

The clusters are also involved in alerting members about bid opportunities (box R.3). Any member can contact the cluster Facilitator, the PWB Official, or one of the clusters' Co-chairs to alert them about the EOI. They in turn will send a memo via email to notify the rest of the members:

Are any members interested in this World Bank Project opportunity?! We have one of our lead companies express a serious interest in leading an EOI bid - and keen to hear from anyone else who is also interested in being involved too! (EENZ-NHNZ Memo, 09 February 2005).

The particular cluster will then start networking to find out if others are interested in looking at the opportunity further (box R.4).

Although there might be interest in the project, there is no firm commitment yet, as many attendants need to consult with other people in their respective organisations. Some companies - mainly the larger ones - may have to go through their own official channels, such as submitting a mini-case to an International Bid Committee for approval of their involvement. In addition, the company which is considering leading the project needs to assess if it is going to get a fair share of the work, as they are taking a huge liability risk by being the lead contractor. It is a balancing act between their interests and those of the cluster.

${ }^{28}$ There is a reference in the Minutes about discussing the issue during the NHNZ meeting in 22 May 2004. 
Being an informal process, the alert of a World Bank/ADB funded project sometimes is received too late and the particular cluster has to rush into action. There have been instances where the decision to lead an EOI was made very close to the deadline, and thus there was no time to circulate a notice. Instead, the lead company contacted individuals directly, or prepared an EOI mentioning both EENZ \& NHNZ clusters to enable other cluster members to be part of the tender if the EOI is short-listed.

Calls for EOIs are generally brief in describing the project; therefore, the interested group needs to research the opportunity further (box R.5). Gathering information to decide whether to submit an EOI or not has to be done very quickly due to the generally short timeframe - usually a couple of weeks - between notification and the due date. The cluster is a useful source of contacts that can provide more information about the project and who is organising it, as somebody in the cluster may know somebody in the country where the project is going to take place. Contacts may be local NZTE officials, New Zealand Embassy staff, local partners, colleagues, and/or known government officials.

A member may invest time and effort in meetings and networking in the early stages of the bid process, but at the end they do not make it into the EOI team, or are assigned a secondary role as backroom support. The risk of not being included is accepted by cluster members as part of doing business in this industry.

Usually though, the situation is the opposite: a small number of interested members realise that they need to recruit the expertise of other cluster members - or even expertise outside the cluster - in order to have a winning team. The initial group will invite others to take part in the opportunity. As one interviewee commented, at this stage the cluster is speculating on the right mix of experts for the particular project, based on a few lines of description from the call for EOI or procurement notice.

Even if the member makes it to the EOI team, there is still no guarantee that they will be in the final team that is going to bid for the project later on if the group gets short-listed. The only exception is the lead company and in particular the person they put forward to be in charge of the project.

At this early stage in the bidding process, it is important for the group to consider a local partner (box R.9). Local partners have the relationships and the local knowledge in the country where the project is going to be carried out. Besides, clusters have found 
that, by including a local partner at the EOI stage, they have a stronger chance of being short-listed.

The clusters have developed quick and cost-effective ways to prepare EOIs based on the growing trust between members and their experience with previous bids (box R.10). The group exchanges templates, CVs, details about their respective organisations, and track record materials in order to assemble the EOI.

The co-ordination for preparing the EOI does not always fall entirely on the leading company. The interviewees provided instances where another interested party offered to collect the material from the various team members and put together a first draft. This allowed the lead company to focus on fine-tuning other aspects of the EOI, making it look professional and consistent. The lead company is ultimately responsible for producing the final EOI document and delivering it to the client.

Not all the members involved in the EOI have the chance see the final document; neither do they get a copy for their records or future reference. The interviewees clarified that this is more an issue of not having time, or forgetting to do so, than not wishing to share the document with the rest of the team:

\section{Have you ever thought about keeping a record of the cluster memory? We are supposed to be, but it is rough; it needs further improvements. Basically what happens is the [...] bid leaders retain copies of all [...] that stuff. The intention is there, the good intention is there. We are just not resource enough to be doing that, as it should be. (Interview Facilitator, 30 August 2004).}

There is no accessible common repository for these materials and templates, and consequently they tend to be scattered amongst cluster members.

The delivery of the EOI is not always a simple task, especially when the client requests a hard copy to be submitted by a particular date. This includes the time it takes to courier the documentation to its final destination and so gives the team even less time to finish their EOI.

What the teams have come up with is to send the material that cannot be e-mailed (such as folders, hard covers, and graphics) in advance to the designated local partner, NZTE official, or NZ Embassy staff who are going to deliver the EOI on their behalf. Closer to the deadline, the team will send the rest of the material electronically. The person/s at the other end will put together all the material from New Zealand, plus the local partner's contribution into the agreed EOI, and deliver the document to the client. There 
are instances where the client accepts an electronic version of the EOI to meet the deadline; however, a printed document should arrive from New Zealand within a few days of the deadline coming due.

It may take some time before hearing anything from the client about which companies or groups have been short-listed based on their submitted EOIs ${ }^{29}$ (box R.13). The client notifies the lead company that their EOI has been accepted and to continue the tender process. The client also attaches a comprehensive document with specifications on how to write the tenders and how they will be scored.

Once the team has been successful in the short-listing, the specific cluster normally plays a secondary role of keeping the rest of the members informed on the progress of the project:

\section{A full update will be given at the EENZ Cluster Meeting now being held next week on the recent intensive bid activity into Turkey and other actions in India, and elsewhere! (EENZ Memo, 25 February 2004).}

It is up to the lead company and its team to prepare their tender and, if successful, negotiate the contract. The cluster continues providing pertinent assistance to the bidding team to increase their chances of winning. For example, the cluster could make a series of actions such as site visits (if staff of a cluster member happen to be visiting the particular market), work closely with local partners and contacts, and use the local NZTE Commission to check for developments on the project.

Full tendering is an expensive exercise (in the order of tens of thousands of dollars), and hence the leader and the team must consider all the facts in regards to the viability of the project and their chances of winning it (box R.14). They examine the details in the specifications required for tender, verify that the clients are requesting something reasonable, and verify that the clients are going in the right direction in terms of methodology and approach ${ }^{30}$. The team will also assess if it would be advantageous to win the bid as leverage for follow-on work (i.e. "preferred provider").

\footnotetext{
${ }^{29}$ It is common that this could take up to 3 months, but there was a case where a client took almost 18 months to reply.

30 For example, the EOI lead by GNS for the consultancy services, for needs assessment to upgrade the emergency management response capacity in Istanbul, was short-listed, but on reviewing the detailed bid requirements it was decided not to proceed to the tender stage. Another example was the feasibility study of the earthquake strengthening of multi-storey buildings in Istanbul. It was after the team was short-listed and got the detailed specifications that they found out that the scope of the work was going to be 369 buildings. This time they decided to go ahead with the tender.
} 
Sometimes the lead company realises that there are not many direct benefits for them in the project, as they would only be performing a small percentage of the total job. However, they may decide to go ahead for the sake of the cluster and other indirect benefits:

\section{So why did you decide to go ahead?}

For the cluster. For the new market. Because it is something we want to get a further reputation. In another way, we had an inexperienced local partner valuable because he is local, a risk because he is inexperienced. In that case, it is actually better for us to be in charge of our destiny. (Interview cluster member, 03 November 2004).

If the team decides to go forward, they will then engage in preparing the tender. There may be some reshuffling of the original team - local partner included - that was put forward at the EOI stage, based on the specifications detailed in the tender document. Some reasons to re-visit the team's composition are: the project is much bigger than first thought; key people in their companies that were in the EOI team are already committed to other jobs; or the initial team does not possess the particular skills for the project or for that particular market. Ultimately, the bid leader would decide the composition of the team as they have seen the "helicopter view" of the project and have a better idea on how to portray the New Zealand expertise.

A decision will be made to send somebody abroad if there is no in-depth knowledge of the area, the market, the local partners and the clients. The team member who goes would look at the site of the project and the area, meet the key decision makers, visit the office which is putting out the tender, and spend time with the local partner. Site visits may have taken place earlier on in the process, when the cluster was tracking new opportunities or before submitting the EOI.

Preparing tenders in the reactive approach (box R.18) is an intensive and usually last around four weeks with a high traffic of e-mails, calls, and meetings. Tenders usually follow a strict format that sets out: 1) the terms of reference for the project; 2) the methodology that will be employed; 3) who will be in the team; and 4) budget and financials. Tenders are now starting to come together faster as the teams have learnt how to do them from previous experience.

Once again, the group exchanges templates, CVs, details about their respective organisations, and track records to assemble the proposal. Financial details such as costs and pricing are also shared. Some of the information required for these tenders has already been shared due to submissions on previous bids, and hence the clusters have 
developed quick and cost-effective ways for the teams to prepare tenders. Nevertheless, introducing somebody else's style may cause some shifting of information, and consequently somebody needs to go through the documents and reformat them for consistency.

The tender that goes to the clients involves two critical components: technical and financial. In the reactive bid process, these two components are delivered in two separate envelopes. The first envelope that the client opens is the technical one. The received tenders are ranked following specific evaluation criteria. An official communication from the client to the winning teams will be sent, notifying them that they have successfully passed the technical criteria. The successful candidates are invited to attend a public meeting where the financial envelopes will be opened.

If the team is notified that it has won the tender, it will start to negotiate the final contract with the client. Within the team, members will revisit the governance aspect of the partnership. Some tenders clearly specify that "associations" must take the form of Joint Ventures or sub consultancy arrangements, and therefore the team needs to formalise their partnership accordingly.

Negotiation is an important milestone. The team is no longer competing against others to win a project. Now it is only the team and the client who are working out the details of the contract. There is a lot of effort put into reaching final agreement. Negotiations will often require the team leader to go abroad for face-to-face meetings.

When agreement on the final contract is reached, the team will start the project. While working on the project, the team gets to know its client better which may lead to discussions of other needs and new projects ${ }^{31}$ (boxes R.25 and R.26). Track records and CVs can be used as support material during these discussions. The successful completion of a project will open up new opportunities for the team and the cluster in that market:

The success of the Bhuj Hospital Project has been built on through a series of now regular marketing visits to India, with the development of key relationships, identification and following up of project prospects (EENZ Minutes AGM meeting, 28 April 2004).

\footnotetext{
31 This was the case in Samoa, where a NHNZ team won the next job - the development of a coastal strategy and disaster management plan - as they were the "preferred provider".
} 
Alternatively, members can start a new cycle of reactive approach by searching and tracking new opportunities from the funding agencies.

\subsection{The proactive project bidding approach}

The proactive approach is the result of the cluster working towards the creation of a new business opportunity. This approach usually takes shape while members of the cluster are working on a project that they have already won - perhaps through a reactive bidding process. Those who are on the ground have been building relationships with their current client, local partners, and Government officials.

Any of these contacts could lead to discussions about other needs, and this in turn can lead to an invitation for the cluster to submit a proposal to meet these new needs. As one of the interviewees mentioned, "In the proactive [approach] we create the situation."

Projects in the proactive approach are normally smaller in scale compared to those from the reactive approach, but they are attractive for other reasons. For example, the client prefers to negotiate with the team as their "preferred provider" rather than putting the project out for tender. In other occasions, the client may approach a small number of candidates and invite them to forward a proposal for consideration. Either way, there is a minimal investment put towards winning the job by the team because it is not following a formal tender approach. In addition, there are direct and open discussions with the clients about the nature of the project and therefore there is less risk to be factored into the costs of the project. This also benefits the clients, as they are getting the job done at a lower price from a team that they already know.

Figure 18 illustrates the activities that the cluster usually carries out during the proactive project bid approach. This is based on the interviews granted by some cluster members who have been involved in these types of bids. 


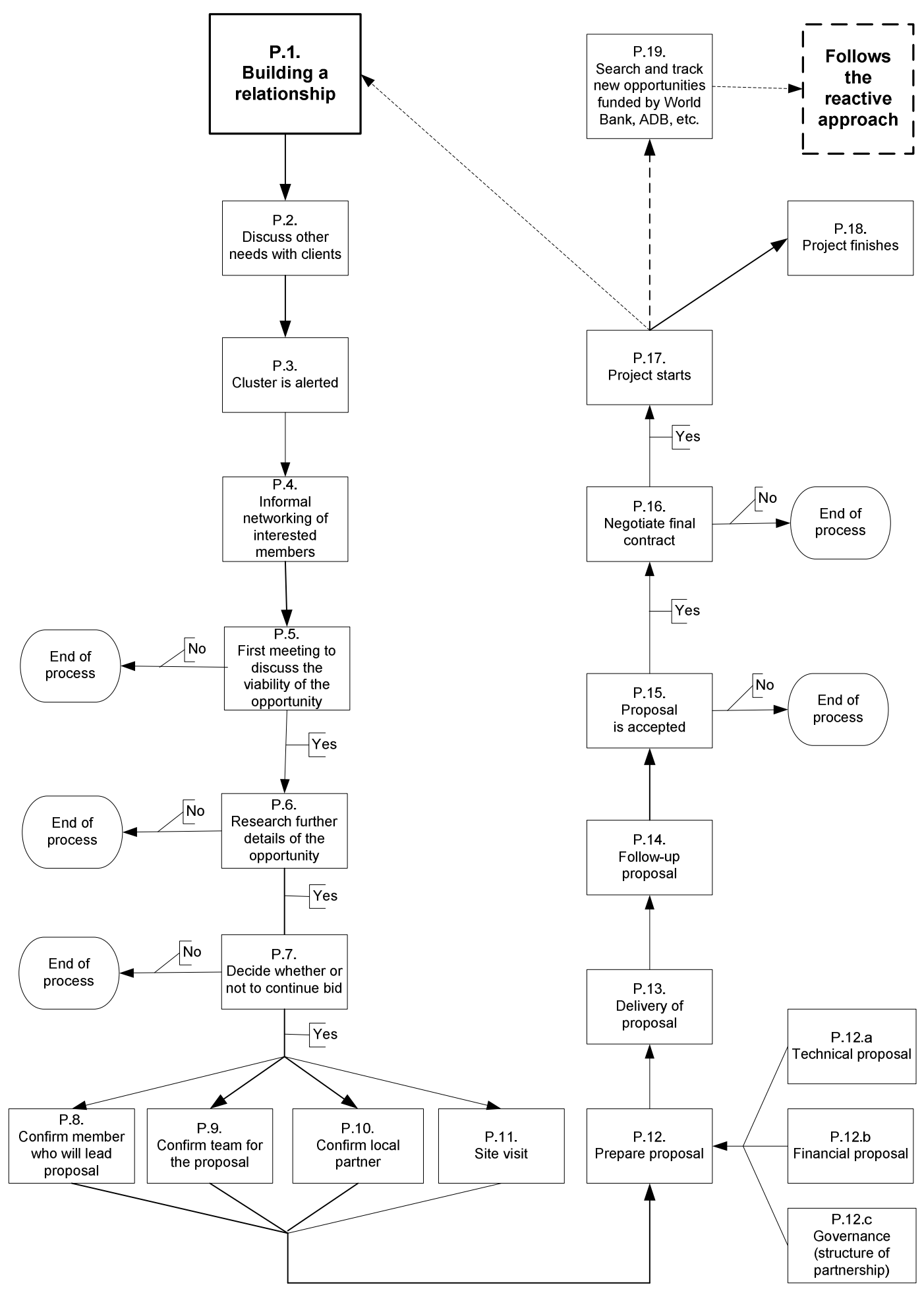

Figure 18. The proactive project bidding process followed by NHNZ and EENZ.

Like the reactive approach, this diagram is the result of interactions between the interviewees and the researcher. The activities have also been numbered from P.1 to P.19 to facilitate the easy reading of the diagram. Again, this numbering does not imply that each activity follows a sequential order in a real bid situation. A detailed description of each of the activities in the diagram can be found in Appendix D. 
The diagram shows that there are fewer activities involved in this process compared to a bid that follows the reactive approach. The diagram also indicates that the team is assessing the viability of the project throughout the process.

Many of the activities that occurred in the reactive approach take place in the proactive approach in the same manner. Examples of this are alerting the cluster (box P.3), informal networking of interested members (box P.4), researching further details of the opportunity (box P.6), getting the team of experts organised (boxes P.8, P.9 and P.10), delivering the proposal (box P.13), and negotiating the final contract (box P.16). Other activities, however, are dealt with slightly differently, such as the cases of preparing the proposal (box P.12) and doing follow-ups (box P.13).

Like in the reactive approach, the proposal will usually have the following content: the terms of reference for the project, the methodology that will be employed, the person in charge of the team, and the budget and financials. Nevertheless, these proposals tend to be more flexible in order to fulfil the client's specific requirements. There is constant negotiation with the client throughout the proactive bidding process, so the proposal evolves over time in terms of cost and scope. Deadlines to submit proposals in the proactive approach are also not as rigid as those in the reactive one.

Based on their past experience, the clusters have developed quick ways to prepare proposals under this situation. The group exchanges templates, CVs, details about their respective organisations, track records, and financial information to assemble the proposal. Like the reactive approach, preparing the proposal will involve going through the documents provided by the rest of the team and reformatting them for consistency. There have been situations where the proposal - or parts of it - has been translated into the client's native language.

There are three activities in the proactive approach that the interviewees pointed out as being more in-depth than in the reactive one: building the relationship (box P.1), discussing other needs with client (box P.2), and following up on the proposal (box P.14).

Building relationships is a critical activity for the cluster because, from these interactions, the clusters may gain extra work:

EENZ are no longer responding to all project tenders. They are proactively targeting countries and development agencies and as a result are being invited to submit bids (Harvey \& Gray, 2003:32) 
As a cluster they organise meetings with key individuals in organisations that tend to issue new projects (such as SOPAC ${ }^{32}$, Red Cross International, and the Ministry of Foreign Affairs), local partners, and Government officials. The clusters have also contributed towards the costs of marketing visits of members to key countries, and they have been involved in official visits to New Zealand from international delegates, such as those from Turkey and China in 2004.

One of the interviewees raised the issue that the cluster could do more to build relationships. Comments were made regarding the need to increase the frequency of emails and phone calls to key people, and complementing such communications with site visits whenever possible.

In the proactive approach there is an open discussion of other needs with the client. As one interviewee mentioned, there is no "veil of secrecy and diplomacy" that usually occurs with the reactive approach. Here, the client has expressed their wish to work with the cluster's team. There is more dialogue and feedback from the client in order for the team to prepare a viable proposal.

The situation is similar during the follow-up after the team has submitted their final proposal (box P.14). Since the client is working directly with them, it is not seen as unprofessional or tactless for the team to ask the client for the status of the decision to accept or reject their proposal.

If the client accepts the proposal, the team will start the project once the contracting paperwork is done. While working on the project, discussions of other needs and possible new projects ${ }^{33}$ could emerge. Alternatively, members start a new cycle of reactive approach by searching and tracking new opportunities.

\subsection{Mapping the knowledge transfer mechanisms}

"Project Bidding" was the name for the clusters' coordinated and distinctive communicative system whose primary purpose was to win a suitable contract. Chapter 6 explains how Project Bidding was broken down into a series of steps or sub-systems, such as: alert cluster, informal networking, and further research opportunity. Each of

\footnotetext{
32 South Pacific Applied Geoscience Commission; this is an inter-governmental, regional organisation that promotes sustainable development in the South Pacific countries.

33 This was the case in Samoa, where a NHNZ team won the next job - the development of a coastal strategy and disaster management plan - as they were the "preferred provider."
} 
these sub-systems involves a sequence of collaborative interactions (i.e. genres) that communicate something in order to achieve an expected action (see Appendix E for the complete list of the genres).

Since many of the sub-systems were either the same or very similar for both approaches (for example, alerting of the cluster, or informal networking of interested members), I was seeing the same genres for the reactive and proactive approaches. Consequently, it seemed practical to merge these sub-systems rather than doubling-up the results. The genres have been organised under the sub-systems they are called upon.

By mapping the genres in Project Bidding, the researcher and the team of practitioners were able to understand the clusters' collaborative practices and how such tasks are structured and executed across time, space, and organisations (Yates \& Orlikowski, 2002). It was the first time the project bidding process was mapped, and cluster members commented on the usefulness of having diagrams depicting the process.

As already stated in Chapter 3, this study proposes that genres are knowledge transfer mechanisms. The Project Bidding system revealed that clusters' experiences and interactions have enabled knowledge to be created and shared by members, through some formal (e.g. cluster meetings, EOIs/Proposals, and memos) and informal (networking, conversations over the phone, personal e-mails) knowledge transfer mechanisms. These mechanisms form part of the clusters' knowledge-base, and many of them are in an electronic form which, according to Markus (2001), could be preserved in an organisational memory system for reuse in future bidding initiatives.

Genre Systems complements Jasimuddin's (2007) decision tree of media use for different transfer situations, depicted in Figure 6. By looking at each genre in terms of purpose, content, actors, form, time, and place, it considers other variables that also have a significant influence in how appropriate a mechanism is for knowledge transfer.

The analysis for Project Bidding identified three sets of collaborative interactions in the clusters: 1) between the team leader and the client, 2) within the bidding team, and 3) between the bidding team and the rest of the cluster.

Collaboration between the team leader and the client is usually structured and formal. Due to the business nature of the relationship, any decision or agreement reached between both parties has to be recorded in a written form. One interviewee explained that the reason for this is to make sure that there are no misunderstandings in regards to decisions made. 
Collaboration within the bidding team is more casual but it remains professional. Due to the small timeframe given for preparing the EOI or the final proposal, there are intense moments of interaction. The team is usually relaxed about documenting its agreements as there is a good level of trust and goodwill within the team. Only when the contract is secured does the team formalise its partnership in some kind of contractual document.

The last set of collaborative interaction is the one between the bidding team and the rest of the cluster. The EENZ or NHNZ is the point of contact for one member to notify the rest about a potential bid opportunity. It is also the entity that triggers the networking efforts to find interested parties to pursue an opportunity:

Robinson Seismic following up on opportunities that [other cluster member] assisted to identify from his APEC meeting visits to Taiwan. This includes quoting for 500 plus bearings for highway bridges. (EENZ Minutes, 04 March 2004).

NHNZ and EENZ also engage in building relationships with local partners, Government officials, and experts from key markets:

Looking forward to seeing you this Wednesday 12 noon for our special lunchtime meeting at Positively Wellington Business re INDIA Earthquake Engineering Natural Hazards Projects Progress \& Opportunities with NZ Trade Commissioner [...] and Deputy Trade Commissioner... (EENZ-NHNZ Memo, 19 August 2003).

Genre Systems also proved to be a useful tool to assist in the evaluation of how appropriate a mechanism is for knowledge transfer, as Brandt-Husman (2001) and Jasimuddin (2007) have advocated. Based on the genres employed in the Project Bidding system, it could be inferred that the tacit knowledge is well served through phone calls and face-to-face meetings. However, managing the explicit knowledge could be made more effective by having a centralised point where team members can deposit and access it, instead of the lead company and the EOI co-ordinator making requests and e-mailing genres backwards and forwards.

\subsection{Areas where ICT could support project bidding}

From the three sets of collaborative interactions that were identified in the Project Bidding system - that is, between the team leader and the client, within the bidding team, and between the bidding team and the rest of the cluster - the last two are worth examining in terms of new ways ICT could facilitate communication, the exchange of knowledge and the availability of information. In particular, searching and tracking new 
opportunities (boxes R.1. and P.19), preparing EOI proposals (box R.10), preparing tender/proposal (boxes R.18 and P.12), and building a relationship (boxes R.25 and P.1) are activities that could be improved.

The activity "Search and track new opportunities funded by World Bank, ADB, etc." (boxes R.1 and P.19) deals with many genres coming from NZTE monitoring services. With the exception of cluster meetings, all of them have quite similar characteristics in terms of actors, form, time, and place. Their main distinction is that they address different purposes.

The interviewees agreed that e-mail has been the appropriate medium to distribute these genres; however, the way it currently operates is not catering to the clusters' needs. Firstly, large volumes of notices tend to be passed on to cluster members without any consideration of relevance. By indiscriminately e-mailing the genres, there is a risk that people will start to ignore e-mails that come from NZTE and hence miss ones with relevant information for them. Secondly, e-mails are good for "on the spot" notification, but the reality for many members is that, if they do not read them immediately on arrival, other e-mails will pile up on top. This increases the risk of a genre detailing a business opportunity being read near its deadline or, worse, not read at all.

Some kind of filtering mechanism could be implemented where members could select the types of genres from NZTE they want to receive. ICT can support this in two ways: by creating an electronic form where users can tick their choice of NZTE genres; and by preparing distribution lists that will e-mail the genres to the individuals. This can be easily implemented, as the types of genres from NZTE were identified during the 'Diagnosis' stage, but careful planning and follow-up would be required to set it up. The issue of not missing an important bid notification could be address by having a more visible "space" to post the announcements of key events (e.g. calls for EOIs) with their respective deadlines, for example an online planner or calendar.

Another two activities with high traffic of genres are the preparation of the EOI (box R.10) and the tender/proposal (boxes R.18 and P.12). Here many templates, CVs, track record, and bits of information are exchanged via e-mail. Requests for additional details are done by phone, meetings, or e-mail. Although the clusters have developed quick and cost-effective ways to prepare EOIs and proposals, they acknowledge that more could be done in this regard. 
For instance, part of the information required to prepare a new EOI or tender could be recycled from previous bids. However, these documents are scattered throughout the cluster and therefore e-mails have to be sent requesting this information. In addition, $\mathrm{CVs}$, track records, and company details are constantly moving backwards and forwards within the bidding team.

A data repository could be the way to address this issue. $\mathrm{CVs}$, track records, and general information about the cluster members could be stored in a centralised data repository. The data repository could also improve the consistency of the documents' formats and could be used to store past EOIs and proposals, and any other material that was produced during the process, for future reference.

Finally, a third activity with high traffic of genres is "Building relationships" (boxes R.25 and P.1). There are meetings, letters, e-mails, telephone calls, and site visits that have been carried out with the purpose of developing ties with external parties, raising the cluster's profile, and creating new business opportunities for the clusters.

Building relationships with clients, local partners, Government officials, funding and development agencies, and international experts is a critical activity performed by NHNZ and EENZ. The cluster concept is a good vehicle to do so, as it offers an image of a consolidated group of firms. New contacts are easy to make, but for them to become helpful relationships for the cluster they need to be looked after and maintained over time. ICT could assist with this issue from various angles. For example, an e-mail distribution list could keep these contacts informed on a regular basis about the clusters' activities. Having a record of key contacts and their areas of expertise, for future reference, is another possible alternative.

The findings from the Diagnosis Stage were used to draft the action plan in the Planning Stage of the study already depicted in Chapter 7. 


\section{Chapter 11: The process of digitising genres}

As already mentioned in Chapter 3, genres are the analytical units used to identify the knowledge transfer mechanisms for sharing and reuse in the clusters. Therefore, attention was focused on how the introduction of ICT transformed some of these genres. Yates and Orlikowski (2002) described genres as stable but not rigid communication structures. Therefore, users are able to create and modify genres over time in order to fit a given circumstance. In the case of NHNZ, the revamping of their website introduced a new medium to share and store some genres associated with the Project Bidding System. Participants in both the Website and Content Task Groups engaged in reviewing the "institutionalised templates" already in place, to make the most of the new technology they now have available.

Given that these genres were converted during the Development Stage to exist in this new electronic medium and take advantage of its functionality, I am adopting the term "digital genre", which has been commonly used by the literature (Rhem, 2002; Ihlström \& Henfridsson, 2005; Pollach, 2006), to acknowledge their new property.

This chapter will first introduce three digital genres created by the NHNZ Task Groups during the Development Stage. Attention will then focus on describing the process that emerged while developing such digital genres for their new IT environment. The final section will discuss the current status of these digital genres.

\subsection{Three digital genres in the NHNZ Project Bidding system}

Genre Systems Analysis was used during the Diagnosis Stage to identify and gain understanding of the collaborative interactions within the "Project Bidding" system prior to the intervention. The analysis showed that some subsystems, such as "Prepare EOI" (box R.10 in Figure 17 and genre details in Table 6), experienced high traffic of diverse genres moving back and forth via meetings, phone calls and e-mail amongst the bidding team.

Three genres with highly tacit knowledge content employed in the sub-system "Prepare EOI" and "Building a Relationship" (box R.25 in Figure 17, or P.1 in Figure 18) were therefore considered to be digitised by the Task Groups during the Planning and Development Stages: the Expert Profile, the Project Sheet, and the Company Profile. 


\subsubsection{Expert Profile}

The Expert Profile is a one-page document about an expert in the natural hazards field who is an employee of a member organisation. Its purpose is to provide a profile of the expert. The content of this genre includes information about the individual's areas of expertise, qualification, contact details, languages spoken, experience, and track record. The Expert Profile is very structured, written succinctly, and uses formal businessoriented language.

Shortly after its inception, NHNZ recognised the importance of its knowledge-base in terms of its members' human resources pool. That is why, at this time, the cluster created and distributed the Expert Profile template for its members to complete. The completed Expert Profiles were then sent to the web administrator who would convert them to PDF format and manually upload each of them to NHNZ's old website. These profiles lacked the flexibility which would make them searchable and easy to update.

The cluster found the Expert Profiles useful for marketing and project bidding activities, and wanted to address the limitations of the original genre using the new database environment. A content management system was set up on the website to enable cluster members to access the Expert Profile template from the website and create/update the one-page profiles themselves:

In the old one, the CVs go on and you can't edit them because they were very simple. Now we have that facility where people can edit them and then download them again themselves. (Interview cluster member, 30 August 2005).

\subsubsection{Project Sheet}

Project Sheet is a one-page document describing a project carried out by cluster members. It provides factual information such as the name of the project, the country where it took place, the beginning and completion dates, which cluster members were involved, and sometimes the value of the overall project ${ }^{34}$. The Project Sheet also provides a couple of paragraphs outlining what the project was about and the specific work done by the cluster members, and an image related to the project for illustrative purposes.

\footnotetext{
${ }^{34}$ Some projects were confidential in nature and therefore the total value was not displayed in the Project Sheet.
} 
In past cluster bidding activities, some members had used their own templates to showcase examples of their work. The intervention provided the cluster with the opportunity to create its own Project Sheet and make it a consistent genre for its members to use. The new Project Sheets can be searched by keyword, country, company, and by type of service rendered.

\subsubsection{Company Profile}

The introduced database and Internet technologies gave the cluster the opportunity to create the Company Profile digital genre, which describes each organisation that is a cluster member. The original NHNZ only listed the member companies in terms of a particular type of service they provided, or by hazard. The Company Profile web page provided information about each organisation in terms of: capabilities and areas of expertise, contact details, a list of its key experts, and a list of key projects it has carried out. The Company Profile is very structured and uses formal business and marketing language.

The Company Profile genre took advantage of ICT by being searchable, and inserting hyperlinks to the relevant Expert Profiles and Project Sheets digital genres. Company Profile also has links out from the portal to the company's own website.

The use of content management systems and a database to manage the Expert Profiles, Company Profiles, and Project Sheets digital genres, instead of static pages, will scale more gracefully over time without adding the overhead costs of opting for static pages. Static pages require the creation of a new page for each additional expert or project, and constant maintenance to keep the links working, whereas the database just requires some set-up work at the beginning to generate the pages automatically. In addition, a database with a search capability allows cluster members to quickly find information about other experts, companies, and projects in a particular field or country for project bidding purposes.

\subsection{The process of creating digital genres}

The three digital genres described in the previous section have differences in their purpose and form. Nevertheless, the data collected during the Development Stage indicated that the Website and Content Task Groups performed a similar set of steps to create them: finding a reference point for the digital genre; defining the social rules for 
the digital genre; embedding the social rules into electronic forms and templates; testing the digital genre; and legitimising the digital genre. Figure 19 illustrates the process of creating digital genres.

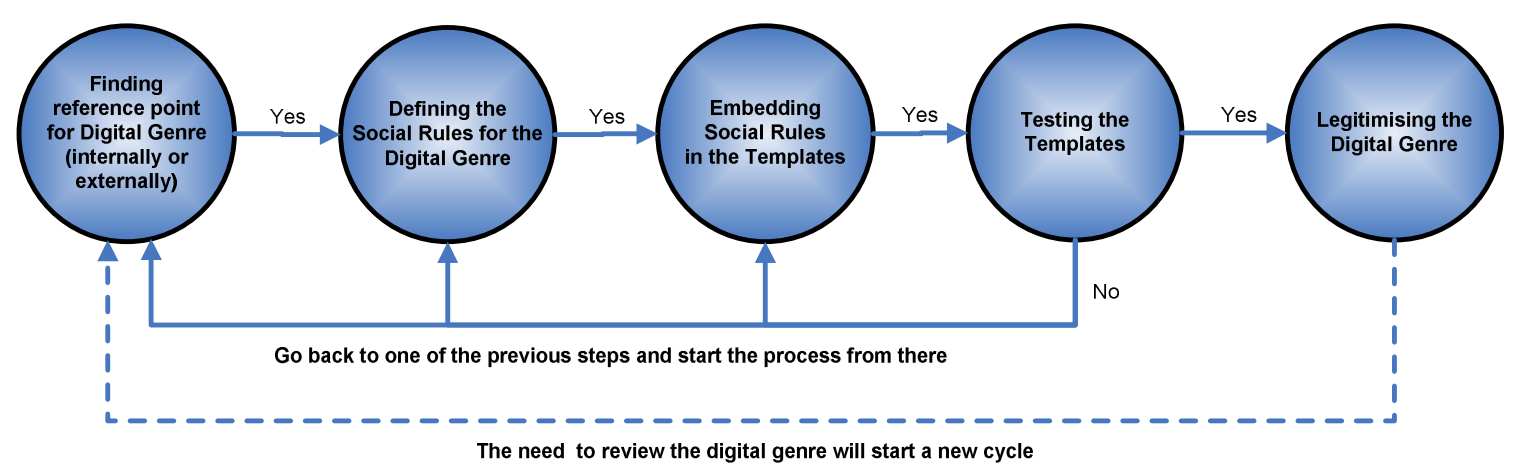

Figure 19. The process of creating digital genres

The following sub-sections describe each of the five steps involved in the process. Given the fact that the Website and Content Task Groups played a similar role and functions during this process, I will use the generic name "Task Group." For this discussion it is only important to acknowledge that a team made decisions about the digital genres on behalf of the cluster; naming the specific team which carried out a certain activity at every instance may cause unnecessary confusion.

\subsubsection{Finding reference point for digital genre}

The initial step in this process involved the search for similar genres available to the cluster in other source media. In this particular case, we mainly collected paper-base and electronic (PDF) versions of CVs, project sheets, and expert profiles. We also gathered a computer-base data model used by one of the cluster members for its own staff profiles.

The Task Group first considered genres within the cluster, such as the case of the Expert Profile where a PDF version already existed. The team opted for genres from cluster members when there were no samples internally. For instance, Project Sheet came about thanks to the contribution of cluster Co-Chair One, who shared examples of one-page description sheets that his organisation had prepared for their projects.

This step is relatively straightforward, since it just collects existing genres for reference. There might be some preliminary assessment of what is at hand and whether the 
community needs to develop something new, but a full assessment of this matter is the focus of the next step of the process.

The first step was consistent with Shepherd's and Waters' (1998) proposal that some digital genres come from existing genres which migrated to the computer and Internet environment. The Task Group team and the website developer were practical people, and looked for a genre in another medium that could be used as a reference point from which to build up the new digital genre.

On another note, this first step provided many examples for Tallman et al.'s (2004) argument of how knowledge is constantly being transferred to the cluster's knowledgebase. Sharing original genres and discussing them not only increased awareness of what it is available within the cluster domain, but also provided the opportunity to learn, from other cluster members, how information could be arranged and displayed.

This knowledge was easy to transfer across due to its component nature; CVs and project sheets are explicit parcels of knowledge. In addition, cluster members share a similar ontology of terms when employing this type of genre. The transfer of such knowledge is not going to disadvantage any cluster member over the rest, as preparation of CVs, company profiles, and project sheets is not their core business activity.

\subsubsection{Defining the social rules for the digital genre}

Defining the social rules for the digital genres was the second step of this process. The community had to agree on how and under what circumstances a specific communication action is appropriate. Attention was focussed on the genre's purpose and content, taking into consideration the functionality of the new digital medium.

The cluster community acknowledged that computing and Internet technologies provide a powerful communication medium to disseminate digital genres; but they were also apprehensive of potential misuse of these genres:

...we need to be sure they [Expert Profiles] can not be tinkered with when taken from the web site. (Email Facilitator, 15 September 2004).

Therefore, social rules needed to be made for the information a particular digital genre should contain, who could view it, who could edit it, how the genre should be used, and what measures should be put in place to ensure its integrity.

NHNZ had a general consensus that information about their companies already displayed in the cluster's website could be used to quickly put together an EOI. 
Attention then focused on defining specific social rules around the new digital genres. In the context of the NHNZ cluster, where they follow the approach of delegating activities to small teams, this step was carried out in two phases: defining social rules by the Task Group, and approving the social rules by the cluster.

\section{a) Defining social rules by the Task Group}

The Task Group on behalf of the cluster started to shape the Expert Profile, Company Profile, and Project Sheet genres by reviewing the fields from the data model collected in the previous step. They decided which details should be included in the new genres, and their respective format, for the first prototype of the system.

During the Planning Stage - and then revisited in more detail at the second iteration of the Development Stage - the Task Group focused on the placement of these genres in the website, and the path that visitors would have to follow to reach them. For example, it was decided that Project Sheets would be accessed through links by region and by country from the Home Page. Company Profile would also have links to the Project Sheets of the specific cluster members who were involved in the project, as well as the type of natural hazard it described or the type of service.

In the first iteration of the Development Stage, the Task Group worked with the web developer in defining three levels of access (Standard User, Primary Contact, and Website Administrator). Depending on the assigned status, a cluster user would be able to edit and create some or all of the genres.

The Task Group was responsible for the final design and layout of the genres. For instance, they defined the fonts, colours, and distribution of elements in the sheets. The Task Group also decided to follow the PDF format to display the Expert Profile and the Project Sheet ${ }^{35}$, and activate the security features provided by this format. By doing so, the risk of people changing these genres was minimised.

\section{b) Approving the social rules by the cluster}

Although the Task Group conceptualised the social rules for the three genres, these were reviewed and approved by the rest of the cluster community during the regular quarterly cluster meetings. The cluster had the opportunity to discuss the associated social rules, and made modifications when necessary.

\footnotetext{
${ }^{35}$ For the time being, the Company Profile would only be displayed as an online page due to the limited resources available for the completion of this project.
} 
For instance, the cluster community agreed that each member would be in charge of the maintenance of the digital genres they produce. Each company would assign a person to be the Primary Contact for issues related to the website. The Primary Contact would be responsible for updating the company details, adding/deleting colleagues from the system, and, when needed, editing information from the digital genres. Individuals whose profiles have been entered in the system would only have access to their own information for keeping their details current.

The study showed that defining social rules is not always an easy task to accomplish even when there are existing genres to use as templates for the digital ones. For example, during the first and second iteration in the Development Stage, the cluster community debated the social rules associated with Project Sheet. Some members suggested following the World Bank specifications to describe a track record. This option, however, involved displaying information that other members considered commercially sensitive, such as the client's address, value of services provided, and the names of staff involved in the project. Eventually, for this round of portal development, the cluster settled for a simple Project Sheet that could be posted in the public area, but would still be useful when preparing EOIs.

In this second step, the cluster's social context can explain some of the decisions that shaped the digital genres, something that Ihlström and Henfridsson (2005) also found in their longitudinal study of online newspapers in Scandinavia. In their case, for example, the internal organisation, the in-house attitude, and the work division defined the level of support, resources, and dedication allocated to online newspapers at three different points in time.

In our case, the voluntary nature of the contributions made by cluster members, their little experience in carrying out this type of work, and the low budget allocated to this project, led to the development of simpler digital genres than first discussed during the early iterations of the Development Stage:

I think the other thing is just the extent - well some of us know - but people failed to recognise on the budget line is the sheer amount of time you got to put in to create the content, to shape the content. (Interview Task Group member, 06 December 2005).

As already pointed out, the cluster has defined procedures on how members should engage them. Addressing these issues produced explicit knowledge about the use of 
new technology for the group, thus enhancing the cluster's component and architectural knowledge-base.

\subsubsection{Embedding social rules in the online forms and electronic templates}

Consistent with Yates' and Orlikowski's (1992) proposition, social rules were embedded in a medium to guarantee adherence to the rules. The Task Group worked along with the web developer to create online forms and electronic templates to incorporate the cluster's social rules, so they in turn could produce the required digital genres automatically. This third step can be demanding on those involved:

I found the sessions that we had out there [with the web developer] quite challenging in a sense. You know, when we had questions and feedback to be answered because I haven't ever thought about those issues before. And then some of them go back to content, such as how much of a short $C V$ did we need, and how many other CVs - a medium, a long one, or whatever - and that was outside my experience or knowledge. (Interview Task Group member, 03 November 2005).

Although the Task Group had a general idea of what they wanted the digital genres to do and look like, they did not anticipate the level of attention to detail that this process entailed, or having to make decisions they regarded as technical, such as defining properties of fields. In addition, this stage showed the importance of having an understanding of the technologies used to embed the social rules.

The social rules were "translated" into programming rules. These programming rules are represented as online forms and electronic templates.

\section{a) Electronic online forms}

The fact that the cluster had decided that each member was going to be responsible for the maintenance of their respective digital genres demanded that the online forms be carefully designed, with clear instructions on how to complete them. The programming rules dictated the fields to be included in the electronic forms and defined their properties - such as word limit for free text fields, or the number of options for drop-down lists - to control the type of input entered.

The Task Group realised the importance of striking a balance between the flexibility of the online forms expected by cluster members, and the quality of the output (i.e. the digital genre). For instance, having "open text" for many of the fields would enable users to quickly enter their data without much thought on how to make them fit to predefined standards. On the other hand, "open text" would increase the 
chances of spelling mistakes, adding several terms for one concept into the system, and not having the genres presented in a consistent manner.

Guessing user behaviour was another issue to consider during the development and design of the electronic forms, in order to effectively guide the users through the data entry process. Sub-routines and error messages were embedded into the forms to alert the users when they enter information in the incorrect format.

Travel and work commitments by most of the Task Group members during the first iteration of the Development Stage prevented proper discussions on how to embed the social rules into the electronic forms for Expert Profile and Project Sheet. The team contented itself with submitting feedback via e-mail in order to make progress. However, at the next step of the process (testing the digital genre), the Task Group realised that the resulting digital genres were not working and they had to revisit this step.

\section{b) Electronic templates}

Based on feedback from a small group of users who tested the system, the Task Group also created electronic templates for the Expert Profile and Project Sheet to show the actual layout of these genres. The templates provided details about the type of information to be collected. This way, the person entering the data would be able to prepare the information before engaging with the online forms.

In our case, the electronic templates were useful tools during the last iteration of the Development Stage, to speed up the process of populating the portal for the launch. At the time, a small group volunteered to enter all the Expert Profiles for the first time into the system; the task of entering the Project Sheets was commissioned to a small web design company. In both instances the electronic templates were a valuable means for cluster members to submit their content to the people in charge of entering the data into the system. Subsequent data entry and maintenance of the digital genres would be the responsibility of the Primary Contact from the respective organisation, as agreed in the social rules.

\subsubsection{Testing the digital genre}

The new electronic forms were tested to ensure that the programming rules were working, that their layouts were user-friendly, and to make certain the system was going to deliver the expected digital genre. Usually it was only when the team reached this point that we found out if the efforts put in the previous steps worked according to our 
expectations. This step involved three set of testers: the Task Group, a small group of users, and the cluster community.

\section{a) Testing by the Task Group}

Testing was first carried out by the web developer and the Task Group, as part of the prototype systems design approach followed for this project. Here the team focused in ensuring that all the needed electronic forms were created and that they captured the social rules.

When a genre needed revision, the Task Group went back to one of the previous steps and started the process from there. For example, testing the first prototype helped the Task Group visualise what the NHNZ Portal was going to deliver, and hence made the team realise that some fields for the Expert Profile and the Project Sheet were not up to scratch. Sadly, in this case, some programming work already done by the web developer had to be reviewed and redone.

\section{b) Testing by a small group of users}

Once the Task Group felt the electronic forms were ready, a second round of testing was performed by a small group of users. The users were asked to interact with the prototype and create their respective Company Profile, Expert Profiles, and Project Sheets.

The small testing group requested some features that would make the system easier to work with, such as deleting unnecessary routines and improving the navigation in the system. For instance, the testers wanted to have the ability to preview the Expert Profile or the Project Sheet from any electronic form. As the system was initially set, the user would have to suspend data entry and go to the public area of the Portal in order to see how their work was taking shape:

Because for me I have to go [...] to Members, the company, and the person that I was entering the data and then download the PDF file to see which ones were the fields that show there. I wouldn't be able to tell from looking at the fields while I was entering the data. So, it is just something that would be useful (Interview Tester Two, 13 July 2005).

Another outcome from this round of testing was for the Task Group to go back to the previous step (embedding the social rules) and create the electronic templates to assist users in completing the electronic forms for Experts Profile and Project Sheet as already explained. 


\section{c) Testing by the cluster community}

Finally, the rest of the cluster community was invited to work with the system to confirm that these were the genres they wanted to use as knowledge sharing and reuse mechanisms. The upcoming website launch during the cluster's fifth anniversary celebrations motivated many cluster members to interact with the system. This new group of testers requested alternative options to fill the electronic forms:

Today the cluster members have started to send examples of track records. The people who are entering this material have encountered another situation... Some of the projects are still ongoing ones and therefore there is no "End Date". Is there a way for the system to accommodate this situation? (Email Researcher, 08 November 2005).

The electronic forms for each of the three digital genres went through several testing iterations by these three set of testers, during and even some months after the Development Stage of the study, before the genres were considered "stable" enough to move on to the final step of this process.

\subsubsection{Legitimising the digital genre}

This step is the end of the journey for the specific genres. The digital genres are made available through the NHNZ portal for members to start using them. As Bawarshi (2000) pointed out, the more cluster members use the digital genres to perform their project bidding activities, the more these are confirmed as the right genres. The digital genres are thus legitimised by the cluster community to be the appropriate communicative actions for particular situations during project bidding.

This final step can also be viewed as a "state of being" for the specific genre. A genre can stay in this state for a long time, until the community feels that it no longer is fulfilling its purpose to a satisfactory level (Yates \& Orlikowski, 2002). A review of the genre is the next point of action. This starts the process of creating digital genres all over again.

Given that I exited the research site a few months after the official NHNZ Portal's launch in November 2005, I was unable to closely follow the uptake of these genres by the cluster. This was also compounded by the fact that bidding opportunities tend to take a while before they reach the point where the cluster needs to call upon these genres. 
Nevertheless, I was able to collect anecdotal evidence that these digital genres have been used for some project bidding and other cluster endeavours in the subsequent years after the Portal's launch. Expert Profile genres were used as part of an Expression of Interest and to prepare a news release in 2006. Project Sheets have been presented to Indonesian Government officials in late 2007 as part of the cluster's effort to build relationships. The online Company Profiles were a reference source in early 2008 for the cluster Facilitator, when he was preparing an EOI to the World Bank for the cluster to be considered as a Procurement Agent for rapid response work after a disaster.

The usefulness of the new digital genres in project bidding activities depends on the efforts by its members in creating further Expert Profiles and Project Sheets, as well as ensuring their Company Profiles reflect their capabilities:

And certainly with a recent expression of interest we put through, I actually went on to the site to look for other partners, looking at their expertise in a particular hazard area. I guess the disappointing thing was that I knew there was one company that had the expertise but it wasn't well reflected still on the website... So I think we got a fair way to go. (Interview Task Group member, 14 December 2005). 


\section{Chapter 12: The development of a cluster's knowledge-base on project bidding}

This Chapter proposes that a cluster has "natural" and "induced" ways to increase its knowledge-base on project bidding. The first case takes place during the normal practices of cluster members working together towards business opportunities over a period of time; whereas the latter case is triggered by a specific event or initiative.

Within the context of this study, the decision to introduce a website and database technology to assist in managing NHNZ's knowledge-base provided an opportunity to explore the role of ICT in increasing this cluster's knowledge-base. The findings of this Chapter are based on anecdotal evidence and the reflections of the participants.

\subsection{The cluster's "natural” growth of its knowledge-base}

The Diagnosis Stage revealed that information on how to bid for international projects or how to address a challenge on a specific market is shared amongst cluster members. EENZ and NHNZ have been a useful source of information about the potential project and/or who is coordinating it, as a cluster member may have relevant contacts in the funding agency or country where the project is going to take place. Contacts may be local NZTE officials, New Zealand Embassy staff, local partners, colleagues, and known government officials.

[Cluster member] noted that [work colleague] recently visited the World Bank in Washington, and appreciated the background information provided to him on the NH-NZ cluster and World Bank links. (NHNZ Minutes, 26 May 2004).

The circle in Figure 20 represents the boundaries of the cluster's knowledge-base in terms of project bidding. Specific bits of knowledge - the small circles - could have been generated by the cluster while pursuing a bid opportunity or been contributed by members for the benefit of the cluster. 


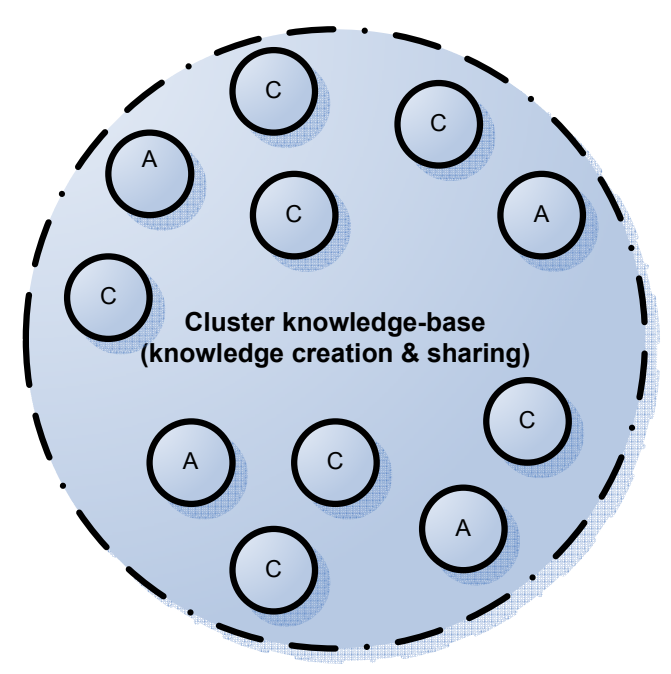

Figure 20: The cluster's knowledge-base on project bidding. (Note: $\mathrm{C}=$ component knowledge; $\mathrm{A}=$ architectural knowledge)

Following Tallman et al.'s (2004) typology, a cluster's knowledge-base can be categorised as component (explicit, transferable, technical, non-contextual, and definable) or architectural (complex, intangible, tacit, highly contextual, and endogenous to the cluster). The genres Procurement Notices, CVs, and Expressions of Interest are examples of EENZ/NHNZ's component knowledge identified in the Project Bidding system. The clusters' respective strategic plans or agreed commission schedules for successful project bids are architectural knowledge mentioned by participants during the interviews.

Evidence from the Diagnosis Stage of this study showed that the clusters have arranged special "spaces" at cluster and team level that stimulate knowledge transfer for project bidding. This substantiates Maskell's (2001) argument that knowledge-base in clusters is the result of horizontal (rivals and competitors) and vertical (business partners and collaborators) dimensions.

Knowledge in the horizontal dimension has taken place when cluster members monitor, discuss, compare, imitate, learn, and add ideas in a common space. For example, there has been an assembling of companies and individuals interested in particular countries and regions in the world, which form Task Groups to watch for opportunities. Two of these Task Groups were: the EENZ Turkey Istanbul Enterprise Network, and the NHNZ-EENZ Asia Earthquake \& Tsunami Recovery Task Group:

... our Cluster Co Chairs last Friday have quickly established a Natural Hazards NZ \& Earthquake Engineering NZ Asia Earthquake \& Tsunamis Recovery Task Group to help co-ordinate and facilitate our cluster initiatives, particularly with respect to liaison with the NZ 
Government and NZAID [...] Thus having a Task Group focused on the recovery will help to provide a focus and central point of contact for Members and other organisations with respect to helping to maximise our input into the development of longer term disaster recovery... (EENZ-NHNZ memo, 10 January 2005).

In addition, EENZ and NHNZ clusters arrange quarterly meetings to network, catch up, and make decisions regarding how the cluster should proceed with particular administrative and/or marketing initiatives. The meetings are casual but structured; they follow an agenda and minutes are kept. It is during these meetings that the team leader of a specific bid will brief the rest of the cluster on their progress:

Tonga World Bank Projects - [Team leader] of [cluster organisation member] gave an update on the successfully won World Bank funded Tonga project. The first stage led by [cluster members] is now well underway, with prospect of possible further work in due course. (Minutes NHNZ AGM cluster meeting, 26 May 2004).

There is a culture in both clusters to circulate via e-mails genres such as the Minutes (which are testimonials of the clusters' evolution of architectural knowledge, their tracking of opportunities, and a record of the clusters' successes), memos encapsulating the learning from a bid, templates for CVs, or networking opportunities.

Knowledge in Maskell's (2001) vertical dimension is the result of co-ordination and communication when members within the cluster join forces as business partners/collaborators to bid for a particular international project. From past bidding experiences, the clusters have gained insights into the material, systems, and procedures they need to develop in order to become a more effective vehicle for their members. Over time, EENZ and NHNZ clusters have enhanced the knowledge-base that could help them improve their next bid proposal, which is reflected in the following passage:

The collaborative experience of making several EOI bids for projects has led to an excellent resource of template and case study material that is able to be adapted for subsequent bids. This coupled with the growing trust relationships between members has led to faster and lower costs of mounting quick response EOIs that previously would have been very expensive for individual companies. (Minutes NHNZ annual general meeting, 22 August, 2002).

The clusters, left alone, are capable of increasing their knowledge-base over time, given a continual flow of market opportunities for them to interact and learn, as depicted in Figure 21 by the increased size of the circles. 


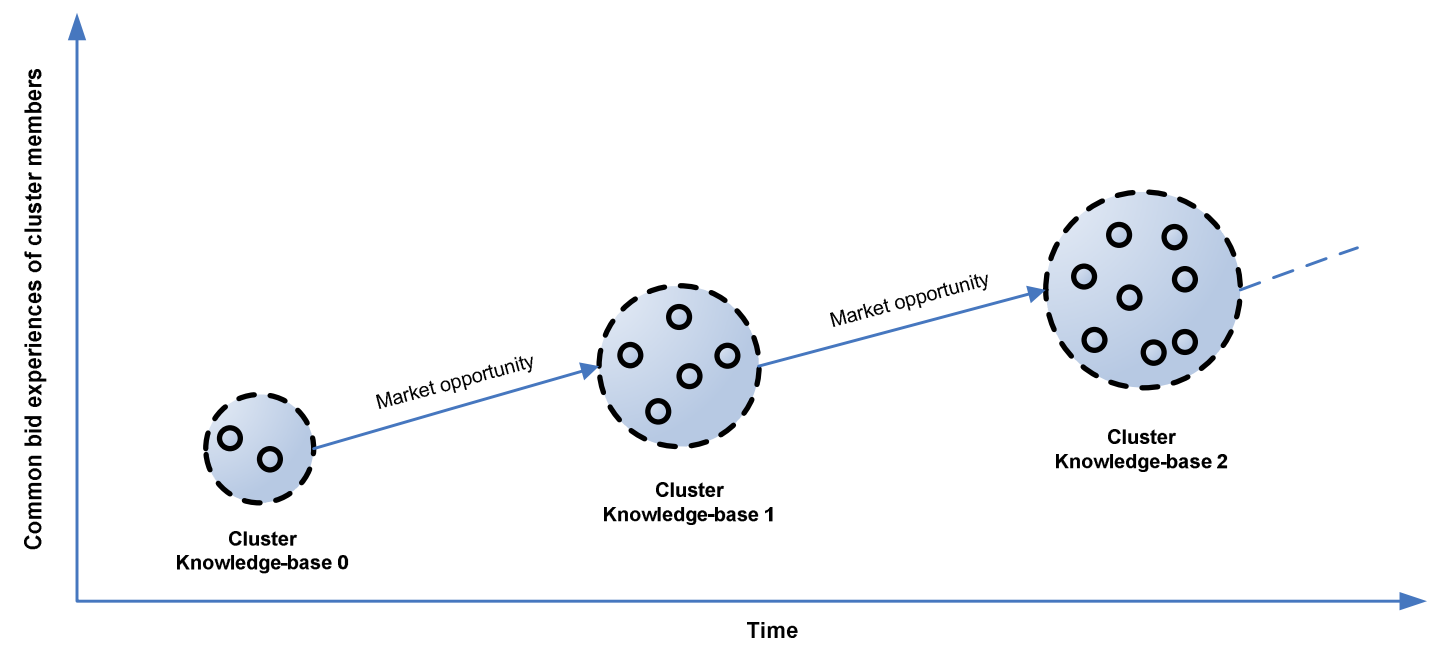

Figure 21: Development of cluster's project bidding knowledge-base over time.

The Diagnosis Stage found, however, that EENZ and NHNZ do not have a "space" to store their respective genres detailing the project bidding knowledge for future reference and re-use. Once the EOI or proposal is completed and sent to the client, cluster members usually move on quickly to tackle the next tender, which is not necessarily cluster-driven $^{36}$.

There is no designated space to store a copy of the EOI, proposal document, or any other material produced during the project bidding process. Only those who participated in the bid would have bits and pieces of these resources. If individual members have not implemented a system to organise the incoming/outgoing material about the specific project - or the cluster in general - at their end, such resources risk getting lost in the rest of their workload:

\section{Have you ever got a copy of the final version or something, or do you} ask for it?

Only if I ask. Even on asking I only have bits and pieces of it, I think.

Looking through my records the other day [...] I could only find fragments of things. (Interview cluster member, 15 October 2004).

Both EENZ and NHNZ have their knowledge-bases dispersed amongst their respective members and other cluster stakeholders, such as the Facilitator and PWB (the regional economic development agency) who may have been involved in the project bidding process.

\footnotetext{
${ }^{36} \mathrm{We}$ have to remember that each cluster member is also pursuing jobs for their own company.
} 
The cluster Facilitator is the stakeholder who may hold a large portion of the cluster's component and architectural knowledge, due to his role in connecting people and prompting members to tackle opportunities. Cluster members and stakeholders contact the Facilitator via e-mail or phone and request a copy - usually an electronic version of some of the resources he holds.

The cluster Facilitator is the closest to what Markus (2001) described as a knowledge intermediary for knowledge reuse. Through his involvement in many of the clusters' initiatives over the years, the Facilitator has authored and collected component material produced by both clusters in terms of EOIs, marketing profiles, drafts of proposals, and even photographs from relationship-building events (such as the case of the Turkish delegation of Government officials visiting New Zealand in September 2004). The Facilitator also has good networks, and architectural knowledge gained from his experiences working with the clusters.

The cluster Facilitator has been partly fulfilling Nonaka's and Konno's (1998) role of "Ba" in an ad hoc manner; that is a "space" where the clusters" knowledge-base has been placed. Nevertheless, it still takes time and additional iterations with the Facilitator before a cluster member has access to the required information; this is particularly critical when the deadline for submissions of an EOI or proposal is close.

The Diagnosis Stage demonstrated that the cluster has a wealth of knowledge in its members' heads and computers which has not been properly stored and/or mapped. Members' experiences and expertise could be overlooked, and advice, the name of a key contact person abroad, or experience of doing business in a specific market may not be considered for a particular project bid.

\subsection{The cluster's "induced" growth of its knowledge-base}

EENZ and NHNZ are continuously increasing their knowledge-base by interacting and learning from their experiences in project bidding. However, there have been instances where the cluster reached a point where it decided to do something about the status quo. This decision then triggered an intensive surge of activity that led to an increase in its knowledge-base. In the case of NHNZ, one of such events took place on 11 March 2004, when the cluster decided to undertake the task of updating its 4-year-old website to make it play a more active role in its core activities. 
The original NHNZ website provided basic information about the organisation, what it offered, and contact details. This was a basic and simple model:

We deliberately made it simple. Partly because we didn't have enough money to make it complicated, but partly because the idea behind it was very simple. Now that we have a bit more money to commit to the website we can bring it up in terms of presentation, to suit a more modern up-to-date presentation. And secondly, the cluster is a bit more mature now: it has track records, it has newsletters, it has quite a few things under its belt. So that's why we can make the website a bit more complicated (Interview cluster member, 30 August 2005).

The Genre Systems Analysis carried out in the Diagnosis Stage identified four subsystems in the cluster's project bidding process that could benefit by the incorporation of new ICT: "Search and track new opportunities funded by World Bank, ADB, etc"; "Preparation of the EOI"; "Preparation of the tender/proposal"; and "Building relationships".

During the Planning Stage of the intervention, the Website Task Group considered the findings of the Diagnosis Stage and thought about how to improve these sub-systems and their knowledge needs for marketing, and for showcasing the cluster's products. They proposed a solution to organise NHNZ's increasing knowledge-base in terms of project bidding by becoming a knowledge repository with Internet access.

With regards to knowledge reuse, the new portal was conceptualised as a tool to facilitate knowledge sharing by capturing, packaging, distributing, and reusing the knowledge already held in the cluster. In the process, three digital genres were created to assist with this: Company Profile, Expert Profile, and Project Sheet.

The new Natural Hazards portal incorporated a content management system and a database, so knowledge that usually is part of an expression of interest (EOI) or a proposal - such as company's capabilities, expert's experience, and track record - could be downloadable following a predetermined template.

This way, bits of knowledge that used to be dispersed in the heads of members and their computers now have a "space" that can be accessible to everybody for future reference. Figure 22 illustrates NHNZ's knowledge-base after the intervention. The outer circle encloses the cluster's component and architectural knowledge. 


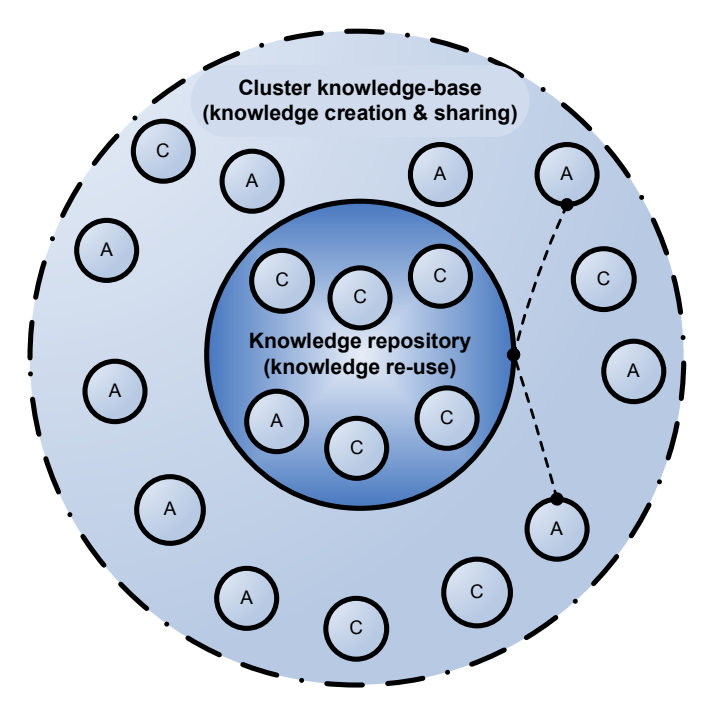

Figure 22: NHNZ's knowledge-base after the intervention.

As already discussed, the cluster's knowledge-base is the result of new knowledge being created or shared by members during project bidding interactions. The inner circle represents the repository where some of this knowledge is now placed for future re-use and reference.

The findings in the study showed that most of the knowledge within this repository tends to be component in nature, such as Expert Profiles and Project Sheets. Some of the architectural knowledge that was represented in a document, such as the Minutes, has also been included in this repository.

Due to their tacit and complex nature, some pieces of knowledge could not be placed inside the knowledge repository. Pointers could be created to indicate where this knowledge resides within the cluster knowledge-base, as represented by the links to the smaller circles outside the knowledge repository. This was partially achieved in the new NHNZ Portal by listing the pool of experts and their contact details ${ }^{37}$ :

I think a couple of times I have gone to the website to get contact details of people. Wanting to contact someone in the cluster and it is just as quick and easy to go to the website and look up the contact details and that sort of thing (Interview Task Group member, 08 August 2006).

\subsection{The role of the Portal in increasing the cluster's knowledge-base}

The intervention was a deliberate act by the NHNZ cluster to organise its knowledgebase. Planning and developing the Portal demanded time, money, and energy from the

\footnotetext{
${ }^{37}$ Other sources of tacit knowledge will need to be identified and linked in a future version of the Portal.
} 
cluster and its members - especially the Website and the Content Task Groups - to change the status quo.

As already discussed, the cluster's knowledge-base would have grown organically, given time and business opportunities to create and share knowledge. However, the portal was conceptualised as a tool to facilitate knowledge by capturing, packaging, distributing, and reusing the knowledge already held in the cluster, such as the explicit knowledge embedded in the Experts Profiles. The Project Sheet and Company Profile, on the other hand, were new knowledge transfer mechanisms that the cluster produced to assist members in their promotion and project bidding activities.

The Portal provided the needed space for storage and re-use of cluster's information and its key capabilities:

... a good example it just happened yesterday for the Prime Minister of Turkey and his diverse delegation. It was a quick way to put some handout material. Simply go to the website. [...] I did the front page, I did the page with the [...] Turkish translation, and the contact details right on the front... the home page and the page with the cluster co-chairs [...] photographs and things like this. And the list of companies, and the list of specialities. [...]. In a few minutes I had a package to photocopy off (Interview Task Group member, 06 December 2005).

Developing a portal was an ambitious plan for a cluster with limited resources, and therefore it is no surprise to see that NHNZ had to do it in stages. That is why the portal design has to be scalable to allow further templates to be incorporated in the future.

Figure 23 is a graphical representation of what the data tell us about the role of the Portal in increasing the cluster's knowledge-base. 


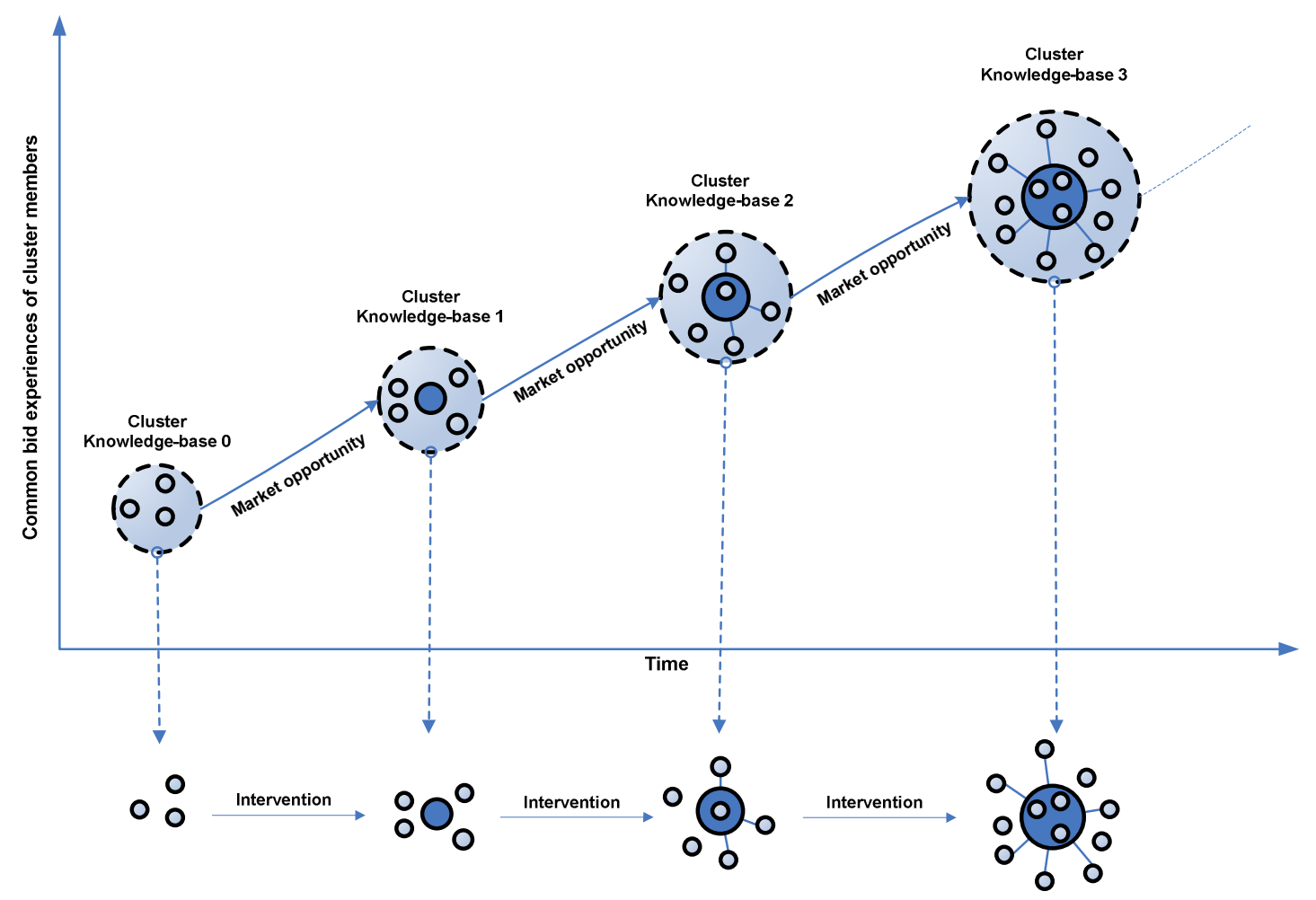

Figure 23. Level of intermediation by ICT due to the interventions.

The Portal prompted the modification of some of the current knowledge-base, to take advantage of the functionality that the ICT technology offered. This is represented by the small circles being placed within the inner circle that corresponds to the knowledgebase repository. The Portal also triggered the creation of new component knowledge, such as the one-page Project Sheets and Company Profiles. This is represented by the increasing size of the inner circle.

Furthermore, the exercise of planning and developing the Portal increased the NHNZ cluster's architectural knowledge on ICT, and how it can be incorporated to provide a better solution for its needs. In particular, those cluster members involved in the Planning and Development Stages learnt about website design as the project progressed. For example, on 06 June 2004, the lead developer received a modified version of the Specifications Document because the Website Task Group had increased its understanding of web technology and wished to implement some of that functionality in the Portal:

...there have been a few items in the document that had evolved and changed as we gain better understanding of what the technology can do. For example, the possibility of using a database to manage the CVs and track projects instead of PDF, e-mailing list and the content management [system]. (Email Researcher, 06 June 2004). 
Members of the Website and Content Task Groups learnt about managing a project of this nature, knowledge that the cluster could find useful in future upgrades of the Portal, or in any other ICT-related initiatives for that matter ${ }^{38}$.

... I learnt that there were numerous ways of dealing with website design, which is understandable because I was not an expert. I suppose one of the challenges we had was demonstrating what we want... (Interview Task Group member, 03 November 2005).

It is important to highlight that the intervention was not isolated from the cluster's complex context:

It has been a very good example of an evolving development. That is because of the range of people that were involved and the ever changing set of circumstances. As we won new work, as we got into new areas of work, we went into new countries, our experiences collectively grew and our specifications for the website probably did change. Initially there were kind of almost conflicting expectations of what the website was for. I am not sure that we could have actually done it any more smoother (Interview Task Group member, 14 December 2005).

While the Portal was being developed, the cluster was gaining knowledge about bidding in the international arena, and what they learnt and understood about the bidding process needed to be taken into account in the cluster's new portal and then embedded into its routines and structures. Using Tallman et al.'s (2004) framework of the types of knowledge at firm and cluster levels, it can be argued that this acquired knowledge was going to feed into the cluster's architectural knowledge.

A series of themes, factors, and conditions emerged while carrying out the intervention, which explains how the NHNZ cluster's knowledge-base was transformed from Figure 20 to become something closer to Figure 22. These findings are the focus of the next Chapter.

\footnotetext{
${ }^{38}$ The Sections "Implications for Practice" and "Update" in Chapter 15 of this study will explain how this architectural knowledge has been reused in subsequent revisions of the Portal.
} 


\section{Chapter 13: Factors that influence the growth of the cluster's knowledge-base}

The action research intervention of this study confirmed that the nature of a cluster does pose a challenge on how quickly its knowledge-base would grow. During my time with the clusters I was able to observe, and discuss with cluster stakeholders, how both EENZ and NHNZ worked on their respective knowledge-bases, in particular through the experience of developing the NHNZ Portal.

While collecting data to answer the two research questions of this study, I came across evidence supporting seven factors that influence the growth of the clusters' knowledgebases: small budget, committed volunteers, active champions, the presence of a project coordinator, a trigger event, supportive economic development agency, and the level of up-take (see Figure 24).

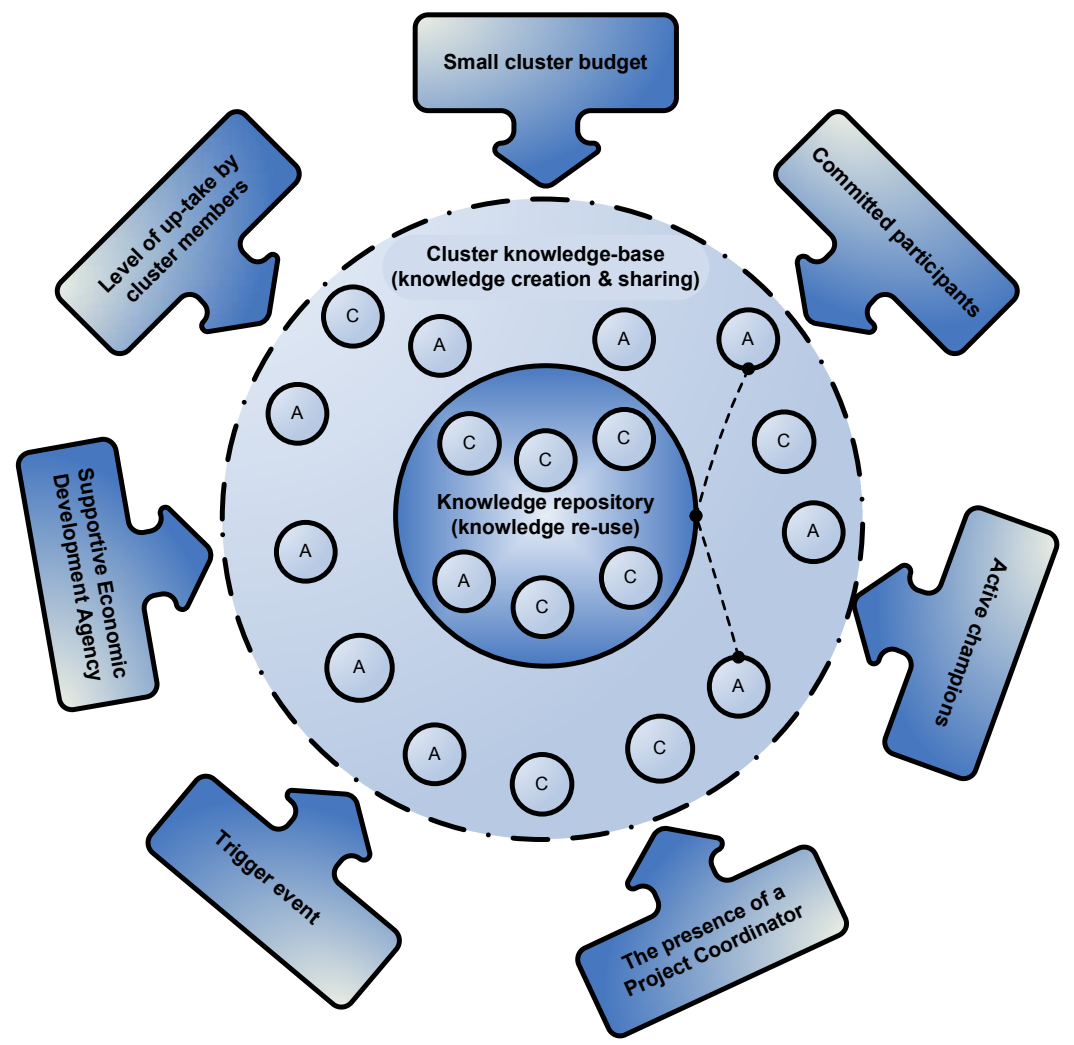

Figure 24: Factors influencing a cluster's knowledge-base.

This Chapter discusses these supplementary findings, which I consider worth reporting. Such factors have been covered in the literature, mainly in the project management field; however I believe there is value in examining them from a cluster perspective. The following sub-sections describe each of these seven factors. 


\subsection{Small budget}

During their initiation stages, PWB (the Wellington's regional development agency) and then the Government funded the services (through New Zealand Trade and Enterprise, NZTE) of a cluster facilitator and setting-up activities as part of their respective cluster development Programmes. However, there was an expectation for the clusters to be self-sustained for the subsequent development stages:

[Cluster Co-chair] noted that the budget this year has been cut back to reflect a tighter income budget estimate [...] now that most income is from memberships and the earlier NZTE and PWB grant funding support almost all at an end. (NHNZ Minutes, 30 November 2005).

By then both clusters were established enough to be able to cover minimal operations ${ }^{39}$ through their membership fees, but they were not yet at the 10-year threshold argued by Porter (1998) for them to become an effective source of competitive advantage.

As already mentioned in Chapter 5, Government support to clusters continued in an indirect manner through other NZTE programmes and funding mechanisms such as the EGD-MD Programme or the Regional Partnership Programme. Nevertheless, these resources are not destined to cover the cluster facilitation, business development, and website development and maintenance - activities that directly and indirectly assist in the management of the clusters' knowledge-base.

Insufficient budget for cluster initiatives was one of the factors leading towards cluster initiative failures mentioned in "The Cluster Initiative Greenbook" by Sölvell, Lindqvist and Ketels (2003). Lesca and Caron-Fasan (2008) pointed out that inadequate budget unable projects to be properly equipped and sustain itself.

The shortage of financial funding for EENZ and NHNZ was a concern raised by Harvey and Gray (2003) in their report to PWB, as it delays the clusters developing innovative product packages and bringing specialised services to market, something that has been on the clusters' drawing boards for a long time. Sufficient resources would also allow the clusters to improve their ability to identify and exploit opportunities, by increasing their knowledge-base about their target markets through good market intelligence and networking.

\footnotetext{
${ }^{39}$ PWB continued providing funding to some cluster targeted marketing initiatives and administration, and it hosted many of the clusters meeting on its premises until July 2007.
} 
As a result of the limited funding, both clusters opted to spread any knowledge-base initiatives in stages over a long period of time:

Given that we are running this on the skin of an oily rag to begin with, as a part-time collaborative venture, there were certain priorities given. Therefore each of them is seen as a progression through over the years. (Interview Task Group member, 06 December 2005).

In the case of the NHNZ Portal, one Task Group member pointed out that the cluster's limited finance hindered the development of a full-scale portal upgrade as desired. The Specifications Document prepared during the Planning Stage detailed how the cluster wanted to a secure repository where it could upload cluster documents. In addition, during the second iteration the cluster community discussed having a more detailed "private" Project Sheet for cluster use only. In both instances, these features were not developed for the first release of the portal. In fact, one cluster member had to fund a couple of jobs to ensure the portal would be a complete unit by the launch date in November 2005. The cluster had to be content with a smaller and simpler solution.

EENZ and NHNZ discussed raising their fees during several of their quarterly meetings in 2005. In addition they revisited their respective policy for Commissions from Projects ${ }^{40}$, which is a voluntary contribution from members who have won a project via assistance from the cluster. Payment of this contribution has not always been forthcoming due to complex circumstances external to this study.

\subsection{Committed participants}

Molina and Yoong (2003) and Braun (2003b) identified in their studies the importance of committed participants to ensure that "things happen" in a cluster. This is critical, as members work for the cluster on a voluntary basis.

One aspect observed by Braun (2003a) was "the lack of time and commitment to the process due to actors' volunteer status" (p. 235). This was also experienced in the Planning Stage of this study, when the first Task Group assigned to do preparatory work to upgrade the website did not follow through. Fortunately, in this case a second Task Group team was convened in the following cluster meeting, which advanced the project.

\footnotetext{
${ }^{40}$ For example the "Range of Scale of Fee Commissions" was moved during NHNZ Cluster annual general meeting in August 26, 2004 in accordance with the cluster's Incorporation Rule 12.3.
} 
Since EENZ and NHNZ are voluntary communities of organisations with a common area of expertise, members can be involved in activities towards enhancing the cluster knowledge-base as much as they are able or desire to. The clusters do not have mechanisms in place to enforce what individuals have offered to do, other than perhaps peer pressure.

Kappelman et al. (2006) and Lesca and Caron-Fasan (2008) noted that project team members with a weak commitment to the project find other daily operational activities to work on that could lead to the postponement or abandonment of the project. Furthermore, in the context of clusters there is an underlying understanding that an individual's priority would be the organisation that has employed them, rather than the cluster; therefore their availability to commit time for cluster initiatives would depend on their workload.

For instance, the NHNZ Content Task Group meeting in 14 October 2005 was scheduled to take two hours, but some of the attendees were only able to stay half of that time as they needed to go to other business engagements. Those who volunteered to work in the two NHNZ Task Groups sometimes took longer to deliver what they were asked, which in turn caused delays in the project.

Even though cluster members - as a collective - are a committed group, people would respond to cluster initiatives depending on how relevant these were for their individual organisation. Consequently, any initiative towards enhancing the cluster knowledgebase would focus on those areas that active individual members find most useful and relevant for their business:

At various points you make that decision [...] where you say, "OK, I am putting this effort in and is benefiting everybody but ultimately I think it's benefiting ourselves. If we have to rationalise it in terms of that effort, then we will do it and I can justify it on the basis of, "it's good for [my company]”. (Interview Task Group member, 14 December 2005).

Given the fact that those involved in the NHNZ Task Group for the Portal development were doing it on a voluntary basis, and that the Portal initiative was not going to bring immediate financial benefits to cluster members, the project was going to move at a much slower pace than that of a commercial initiative:

In terms of time scale of course, being a volunteer organisation it sort of crept along. If there were big bucks involved directly to members, then it probably would've gone much quicker (Interview Task Group member, 03 November 2005). 
Another reality of volunteer work is that people come and go at different stages of the development, and this is disruptive for any cluster initiative (Molina \& Yoong, 2003). There is a feeling of lack of continuity for the project and lack of context for the individual. This was the case in a couple of instances during the NHNZ Portal development:

I'm conscious of the fact that I came into the process after a fair amount of discussion and don't have all the information, so some of my suggestions might not be appropriate. (Email Task Group member, 15 October 2005).

\subsection{Active champions}

The role played by the NHNZ Co-chairs as the champions of the Portal was critical. Without their involvement, the NHNZ portal revamp may have not taken off. Supportive champions were identified by Molina and Yoong (2003) as a key factor to ensure knowledge sharing initiatives, and thus facilitate the increase of the cluster's knowledge-base. Champions lead the rest of the group with their vision, enthusiasm, and example. In other words, these leaders are risk takers, collaborative, and they strongly believe in the cluster idea, which are some of the attributes that Henton, Melville, and Walesh (1997) considered essential to good leadership in the new economy.

Although not a requirement, champions do tend to come from top management, as they have the position and personal relationships to influence and bring together the resources needed to ensure completion of the project (Sipior, 2000). This was confirmed by the fact that the first Website Task Group formed in December 2003 to do preparatory work did not follow through. It was only when the two cluster Co-chairs got involved and led the second team that the Portal project took off.

The two Co-chairs also volunteered human resources from their organisations to perform several tasks for the project, such as testing the prototype and entering data for the Expert Profiles. When we realised that a couple of features for the new portal were not clearly stated in the initial contract, one of the Co-chairs met with the website developer to discuss the issue and negotiated a second agreement. This Co-chair was successful in securing the extra funding from his organisation to cover the additional work. 
Both Co-chairs actively participated in providing feedback throughout the development process and at least one of them was present during the meetings with the web developer ${ }^{41}$. It was interesting to note that when one Co-chair's participation started to decline, the other Co-chair became more active, thus keeping the project moving forward, slowly but surely. Even the cluster Facilitator showed leadership by "making things happen" at various instances of the Portal project when there was a need to fill gaps, such as looking for volunteers to upload Expert Profiles.

This transfer of leadership between individuals in the cluster was also observed by Zhang and Faerman (2007) while examining the dynamics of leadership in the development of a knowledge sharing system. The authors propose that leadership can be distributed amongst several individuals during the development and implementation stages of a project, rather than just being vested in a single person for the duration.

Finally, the Co-chairs were enthusiastic supporters of the project at the cluster meetings. For example, during the cluster meeting that took place on 25 May 2005, Co-chair Two shared his belief that the website was going to be a useful tool for members when they are abroad, as they would have access to Expert Profiles, Company Profiles, and Project Sheets from the website at "the tip of [their] fingers". By actively and publicly supporting the project and showing interest, top management validates the work carried out by the project team (Lesca \& Caron-Fasan, 2008; Nah \& Lau, 2001).

\subsection{The presence of a project coordinator}

Project champions are good in "seeing the big picture," but they do not necessarily have the time to coordinate the project at an operational level. This is particularly true in the cluster context, when they have to justify to their employer why they are spending time on a cluster initiative rather than on chargeable time with clients (Molina \& Yoong, 2003).

Nah and Lau (2001) pointed out that a person or a group of people needs to be responsible for driving the project. Developing the NHNZ Portal showed how important it was to have somebody who kept the project moving along, as the following comment made by one cluster member points out:

\footnotetext{
${ }^{41}$ With the exception of the meeting on 26 October 2004, where only the web developer and I attended.
} 
The problem with the cluster is that it is a sort of volunteer group, isn't it? People are very stretched on doing good-will work, I suppose... [...] to push the boundaries on it requires a considered effort by [...] someone like an enthusiast. (Interview Task Group member, 03 November 2005).

The literature usually allocates this coordinating role to a project manager (Kappelman et al., 2006; Sutterfield, Friday-Stroud \& Shivers-Blackwell, 2006). In the case of this study, the Facilitator and I carried out the role of project coordinator. We did not consider ourselves to be proper project managers as we did not perform many of the functions this position entails, such as planning, defining tasks, and estimating required effort. However, we reminded the Task Group teams - and the rest of the cluster as the website launch approached - to provide feedback, arranged meetings, and prepared notes for the rest of the team to make decisions. It was a time-consuming and demanding job, where patience and perseverance were key ingredients.

My main motivation in playing this role was my desire for the project to succeed, as it was becoming a major component of my research. Even though from an academic perspective I could still deliver insightful findings from a failed project, being able to take this website through to completion would be a more enriching experience to study.

\subsection{Trigger event}

I propose that a trigger event is a factor that influences the growth of the clusters' knowledge-bases. Setting up an important event as a deadline for the project helped to trigger action in completing the project, and creating and transferring knowledge. For the NHNZ cluster, this event was on 11 November 2005, when the new website was going to be officially launched by the New Zealand Prime Minister, Rt. Hon. Helen Clark, during the cluster's fifth anniversary celebrations:

This process, especially with having a launch with the Prime Minister and everything, it made it a real focus for our members to actually complete getting the material ready in time, then we helped to massage it into shape [...] And there is nothing like once the date was set for the event to move people! (Interview Task Group member, 06 December 2005).

The portal project started on 25 May 2004 when the second Website Task Group was formed during the cluster meeting, but it dragged on for a long time due to delays by the Task Groups and the web developer. However, once this deadline was set by the cluster 
- and the day for the official launch was drawing near - a surge of activity took place to create the component and architectural knowledge associated with the Portal.

The cluster secured funding from Positively Wellington Business to contract the services of a small web design firm to edit and upload the Project Sheets into the new Portal. Individuals volunteered their time to do some data entry. In addition, the Content Task Group scaled down the portal's size and features to ensure that it was going to be operational for the launch.

Cluster members started to be more involved in the project. They provided the requested information about their companies, staff, and track records to populate the digital genres; they even supplied content for other web pages. Some cluster members accessed the private area of the portal and tested the online forms and functionality.

The reality of the cluster's context meant that things had to be kept small and simple in order to deliver a good outcome. There is still more work to be done to the NHNZ Portal in terms of content and functionality to make it work as a "true" portal.

\subsection{Supportive Economic Development Agency}

The cluster literature has pointed out the important role played by economic agencies in supporting clusters at the regional level (Bergman \& Feser, 1999; Lagendijk \& Charles, 1999). Positively Wellington Business (PWB) is the Economic Development Agency for the Wellington region. Throughout the years, PWB (and its predecessors WREDA and CDA) has assisted the clusters in building their knowledge-base with various forms of financial, in-kind, and staff assistance.

Despite funding reductions for the clusters in the past years, PWB still approved small grants to partially assist them in marketing and bidding initiatives through their Active Marketing Programme. These grants enabled the clusters to contract experts to assist them in developing key architectural knowledge, like strategic and marketing plans, and hire man-power to speed up the creation of component knowledge, such as the Project Sheets in the NHNZ portal. Grants were also issued to cover marketing bid costs (such as the case of three Tonga World Bank projects in 2003), update brochures, building relationships (such as supporting preparations for the Turkey and Tianjin delegations in 2004), and provide the services of the cluster Facilitator.

Another way that PWB supported the clusters has been in kind. For instance, the NHNZ Minutes of 26 May 2004 recorded that PWB delivered 100 CD copies of the new 
NHNZ promotional PowerPoint presentation for members to use and distribute at their convenience. PWB has also printed small quantities of material for the clusters at various points in time.

Many of the NHNZ and EENZ meetings took place at PWB's board room. PWB is centrally located in Wellington's CBD, relatively close to the offices of many cluster members. They contributed with refreshments before and after the meetings, which encouraged many members stay, network and transfer knowledge. PWB also provided communication technologies to facilitate cluster members attending the meetings remotely:

There may be an opportunity for you to join the meeting via teleconference. If any one would like to do this - please let me know by Tuesday 30 November and I will send you instructions. (Email PWB Liaison, 23 November 2004).

In these instances, PWB facilitated the physical "space" at cluster level that stimulated knowledge creation and sharing for project bidding.

PWB showed its commitment in supporting the clusters by assigning a Sector Manager to liaise with them. The staff fulfilling this role generally attended the cluster meetings and channels requests and communications between PWB and the clusters. Other staff at PWB assisted the clusters in preparing news releases and - although not directly related to knowledge-base - accounting book-keeping.

For the case of the NHNZ portal development, the Sector Manager volunteered some of her time to co-ordinate the entry of the Expert Profiles in the system. With her assistance we prepared the Letter to the Primary Contact and the Letter to the Standard User to distribute to cluster members once their details had been entered.

The PWB Liaison volunteered to be a member of the Launch Task Group which was to organise the official launch of the portal at the fifth anniversary celebrations. The Sector Manager and PWB worked with the NHNZ cluster to secure New Zealand's Prime Minister, Rt. Hon. Helen Clark, and Wellington Mayor Kerry Prendergast to attend the event.

\subsection{Level of up-take by cluster members}

Finally, and closely related to the level of commitment, is the level of take-up by cluster members for any initiative to increase the cluster's knowledge-base. The literature indicates that all stakeholders need to be engaged in the project for them to make it a 
high priority and be willing to participate towards its success (Kappelman et al., 2006; Lesca \& Caron-Fasan, 2008). Actions to encourage this engagement are: keeping everybody well informed on the progress on the project - this also helps to manage expectations - (Nah \& Liu, 2001), and giving them the opportunity to provide feedback and contribute to its development (Andersen et al., 2006).

In the case of the Portal development, NHNZ members received progress updates at every cluster meeting. Cluster members were also shown prototypes of the Portal during some of these meetings, and their impressions and suggestions to improve the solution were noted. Once the Portal was ready to be populated with content, cluster members responded to the call to submit content for the Company Profile, Project Sheets, and Experts Profiles as preparation for the website official launch, but many of them did so only after follow-up calls by the PWB Liaison or the Facilitator.

The reality is that a proportion of both clusters' membership bases is comprised of passive members. Due to this, any cluster initiative is not expected to get $100 \%$ buy-in from members:

I mean, it is always nice to have the participation of everybody, but the reality is that in this kind of thing [developing the portal] is that it is always a handful rather than everybody to motivate it through (Interview Task Group member, 14 December 2005).

The cluster Co-chairs and the Facilitator have indicated on various occasions that, as long as a majority of members are participating and being supportive of an initiative, they would consider it successful. 


\section{PART 4:}

\section{DISCUSSION AND CONCLUSION}

Part 4 discusses further considerations associated with carrying out this research and the conclusions of this dissertation. 


\section{Chapter 14: Considerations associated with the study}

This chapter discusses three sets of considerations I was confronted with while carrying out the study: executing action research, ethical issues, and the credibility of the study.

\subsection{Considerations when executing the action research study}

Action research has its challengers. The idea of developing theory through practice has been questioned by researchers who view action research as a "fragile" methodology (Burns, 1999). McNiff (1988) mentioned that some critics argue that action research is inherently subjective, and consequently it is unreliable and cannot be universally tested. Levin and Greenwood (2001) pointed out that due to its narrative form of reporting findings, action research is viewed by some academics as mere "storytelling."

Baskerville and Wood-Harper (1996) also mentioned that action research has been tagged as "consulting masquerading as research" (p.241). Furthermore, as many action research enquiries are based on reflection of individuals' own practices (e.g. teachers and nurses), claims are raised that action research is just a procedure to improve professional performance (Burns, 1999; McNiff, 1988). All these arguments have caused slow acceptance of action research into mainstream research.

Nevertheless, this trend is changing. In recent years a gradual increase of studies in tourism (Braun, 2003), information systems (Wellington, 2002; Kock \& DeLuca, 2007), and training (Davison, 2001) to name but a few, have opted for action research as their methodology. The findings and proposed theoretical frameworks of these studies have enriched the knowledge-base in their respective fields.

Reason and Bradbury (2001) indicated that action research does not fit with the traditional concept of academic research. Action research follows a different purpose, is based on different relationships, and has a different way of creating knowledge. Therefore, action research studies face a set of issues that traditional academic research may not be exposed to - or at least not to the same extent.

A search of the literature and my own reflection has identified the following action research issues: outcome failure, availability of participants, issue of power, and managing expectations. The sub-sections below will describe each of these issues, and how I handled them to minimise their effects in my study. 


\subsubsection{Outcome failure}

The major risk I took by employing action research was failing to deliver a satisfactory solution for the clusters and/or failing to contribute to the IS knowledge. This shows the delicate balance that this study must have in order to be able to serve "two demanding masters" (Kock \& Lau, 2001:6). Baskerville (2001) labelled this risk outcome failure, which can be first-degree outcome failure, or second-degree outcome failure.

A first-degree outcome failure takes place when the action research inquiry is unable to resolve the practical problem. For Baskerville (2001), this is worse than the seconddegree outcome failure due to the fact that any theoretical contribution is tightly related to the resolution of the problem; if the problem is not solved, then it is difficult to validate any knowledge derived from the inquiry. A first-degree outcome failure affects both the researcher and the host organisation.

Other action researchers, however, do not share Baskerville's (2001) views that a firstdegree outcome failure cannot contribute to knowledge enhancement. For instance Olesen and Myers's (1999) action research project, which aimed to produce organisational change in the areas of communication and coordination by introducing a groupware system, failed in the sense that it did not meet the researchers and management goals. In their study, the organisational culture inhibited the expected changes, and the groupware system ended up being used in a manner that maintained the status quo. Even under these circumstances, Olesen and Myers (1999) argued that knowledge was generated which benefited both practice and theory. Participants achieved valuable insights into their organisation's culture and norms. Their contribution to theory focused on how strong resistance to change could be, and the possible challenges faced when implementing communication and collaboration technologies in an organisation.

A second-degree outcome failure occurs when the action research inquiry finds a solution for the practical problem, but does not provide any significant contribution towards knowledge. In my case, this could have happened if the study developed appropriate information systems for the NHNZ cluster to support knowledge transfer in their project bidding processes, but the lessons that emerged from the study were not rich enough to generate new themes and patterns for knowledge enhancement.

To manage outcome failure, Baskerville (2001) suggested being vigilant while executing the project to recognise early signs of problems. He listed four types of underlying risks to look for, which left unchecked would lead to outcome failure: 
completion risk, domination risk, detachment risk, and abstraction risk. Table 7 describes these four risks.

\begin{tabular}{|c|l|}
\hline Underlying risk & \multicolumn{1}{c|}{ Description } \\
\hline Completion risk & $\begin{array}{l}\text { The researcher leaves the site too soon and therefore does not achieve a sound } \\
\text { understanding of the actions and reactions that took place. This occurs because } \\
\text { the researcher misinterpreted the first indications of improvement as "success" } \\
\text { and declared the study was completed. From the practical point of view this may } \\
\text { lead to short-lived solutions; while from the theoretical point of view, this lack of } \\
\text { understanding may lead to the development of flawed models. }\end{array}$ \\
\hline Domination risk & $\begin{array}{l}\text { The action research takes place without the right collaborative balance between } \\
\text { the researcher and the problem-owners. If the study is dominated by the } \\
\text { researcher's drive to generate knowledge then there is risk that the actions taken } \\
\text { overlook the urgency of solving the immediate problem. On the other hand, if the } \\
\text { problem-owners' interests in fixing their problem dominates the study then there } \\
\text { is risk of generating a weak theoretical explanation. }\end{array}$ \\
\hline Detachment risk & $\begin{array}{l}\text { The researcher is not committed to either solving the immediate problem or } \\
\text { exploring theories to explain what is going on. }\end{array}$ \\
\hline Abstraction risk & $\begin{array}{l}\text { Involves the challenge of balancing action and reflection in action research. The } \\
\text { researcher is tempted to spend time examining and reflecting about the problem } \\
\text { and its complex setting to reduce the risk of poorly defined actions. However, } \\
\text { extensive periods of abstraction may contradict action research's swift iterative } \\
\text { nature of testing, evaluating and refining imperfect theories until no more changes } \\
\text { are required. }\end{array}$ \\
\hline
\end{tabular}

Table 7. The four underlying risks that could lead to outcome failure (Source: Baskerville, 2001).

Fortunately, my study did not suffer from first-degree outcome failure. The participants and I delivered a practical solution: a first version of the NHNZ Portal. Although the solution needs further development to reach its full potential as a true portal, the participants found it to be an improvement on their "old" NHNZ website.

Furthermore, the clusters also gained understanding of their own project bidding processes (proactive and reactive approaches) as a result of my analysis during the Diagnosis Stage of the study:

Maria Molina gave an update on the first stage of her action research study re international bid work by the EENZ and NHNZ business clusters. She has documented the various approaches, providing a useful knowledge base as part of on going reviewing of [...] processes to improve cluster international bid initiative results. (EENZ Minutes, 17 February 2005).

I also avoided second-degree outcome failure. While working on the practical solution I collected data that enabled me to contribute to the IS stock of knowledge. Firstly, I applied Genre Systems as a tool to map genres in a business process that takes place in 
an inter-organisational context. Secondly, I proposed that digitising genres follows a five-step process. Thirdly, I described how a cluster's knowledge-base grows over time, following what I called natural and induced approaches. Fourthly, I explored the role of ICT in knowledge transfer. Finally, my study identified factors that influence a cluster's initiative to enhance its knowledge-base.

There were, however, instances towards the end of the Development Stage when addressing the practical problem became the predominant activity; this made me question if there was sufficient interesting material to allow me to develop theories:

Sometimes I wonder if I have enough time to cover the study and if I have enough material to make this study academically enough. At this stage everything feels so blurry! Not sure how much of a breakthrough my study is going to be. (Journal Entry, 27 July 2005).

I kept thinking of the "trap" that many action researchers warned of regarding putting too much focus on solving the practical problem and not enough on contributing to the enhancement of knowledge. This was raised during my conversation with my Supervisor, who provided much appreciated guidance.

With regards to the four underlying risks for outcome failure mentioned by Baskerville (2001), I found that domination risk and detachment risk did not play a relevant part in my study. The cluster participants and I worked well together towards the achievement of the practical solution:

The meeting went very well. I actually took a secondary role. [...] I am there to support and learn. However, I asked questions and contributed a little. I got up and used the whiteboard to "draw" the pages after [Task Group member] had done it first - that was a good idea. (Journal Entry, 19 March 2004).

As a researcher, I was committed to both solving the practical problem and exploring theories to explain what I observed during my time with the clusters. Cluster members were supportive of my efforts in developing further theories, by allocating time to review interim summaries of my findings, granting me interviews, and by simply being interested in my progress.

Completion risk was the prevalent one in my study. I had a limited time to be involved with NHNZ, which did not neatly fit with the slow pace of how things occur at the cluster. To overcome this, I collected the final impressions from participants up to seven months after the official launch of the portal, and I followed-up their progress - but not as a researcher - in the subsequent years. This allowed me to capture anecdotal 
evidence of the type of use the portal was getting for project bidding and the commitment by the cluster to continue improving the portal that fell outside my time with the cluster as an action researcher.

Abstraction risk was another issue in the study. I collected a large amount of data for each of the stages in intervention. I was aware that processing and making sense of this material would take a long time. However, the cluster was not going to wait until I had completed my analysis to address their practical problem, and therefore I needed to move on to the next stages of the intervention.

For instance, on March 2004 a cluster Task Group was ready to work on revamping NHNZ's website. This project had the information technology component for an IS study and it aligned with the Diagnosis Stage preliminary findings. I could not stay behind until I completed my analysis on project bidding before becoming part of this team. I had to take this opportunity despite it not being neatly timed.

Given the nature of action research, I believed we have to accept the fact that the academic timing is not the same as the practitioner's one. Therefore, as academics, we have to be flexible and accommodate our schedule to be able to tackle good research opportunities. Once I exited the site I focussed on processing the data and theory development.

\subsubsection{Availability of participants}

During the intervention I worked with people who had volunteered their time and services for the cluster. I accepted the fact that cluster members were free to come and go from the Task Groups assigned to develop the NHNZ portal. For instance, participants left temporarily (e.g. business trip) or permanently (withdrawal from the Task Group), and their replacements brought fresh views about the direction of the project.

My own availability and that of the developer and cluster members impacted on our progress, given the fact that the study was not in a controlled environment. We had personal and professional commitments at different times that needed to be accommodated within the parameters of this study. Unfortunately, once we came back to work on the project we needed further time to get familiar with how things then stood. Consequently, the study took longer than anticipated, as decisions to address the practical problem could not be made until most of us were present and ready to consider the facts or to do the job. 
The availability of participants was out of my control. Their willingness to collaborate in this study depended on their good-will and desire to achieve a practical solution to the problem.

The only measure I could take to minimise the negative effects of participants' availability in my study was to ensure that the time they allocated to the project focused on moving it forward rather than catching up. For this reason, I opted to play the role of the "project coordinator" in the project. I prepared material for their consideration, arranged meetings, sent reminders, and kept participants informed while they were away, thus keeping the momentum going:

A quick note to let [web developer] - and those who could not make it to yesterday's cluster meeting - know that the Website presentation went very well [...] During yesterday's meeting, the cluster members were happy with the progress of the project. Two key questions were asked: 1) when the website will be ready for them to start entering their data; and 2) when it will be ready to launch it. (Email Researcher, 11 March 2005).

\subsubsection{Issues of power}

Consistent with the advocacy/participatory research paradigm proposed by Creswell (2003), action research entails a degree of involvement by the researcher and the problem owners. As a researcher, I played a more active role than being just an observer or interpreter of what was happening in the clusters, something I found very appealing. Nevertheless, there is a price to pay for such an allowance: the researcher gives up a degree of authority to become more of a collaborator, while participants are no longer passive recipients of the research process (Chisholm \& Elden, 1993; Sommer \& Sommer, 2001).

A recent view on this matter proposed by Avison et al. (2007) puts power as the umbrella concept from which the following themes are exercised: authority, which comes from organisational (position) or institutional (degree, training, expertise) structures; resource rights, being able to assign people, funds or material to a particular project; influence, as the result of social attributes or charisma; and politics, as a result of exercising strategic processes.

For Avison et al. (2007), each action research study would have a unique mix of these power themes due to its context. The authors put forward three aspects to be considered when determining the appropriate power structures for the study: the initiation of the 
action research project, the determination of authority for action in the research project, and the degree of formalisation of the project.

Initiation refers to who started the action research project: was it the participants, the researcher, or collaboration from both? This intervention fits best as a collaborative effort. The study evolved from previous work I had carried out on clusters, which gave me the opportunity to meet NHNZ and EENZ members and their Facilitator. Both parties - the researcher and the practitioners - acknowledged the synergies of working together. Nevertheless I did not have complete freedom to "pick and choose" the problem to study (Baskerville, 1999), as it had to be something that the cluster was interested in tackling. This lack of control could make the study lean towards searching for a practical solution and neglecting the knowledge creation aspect.

The second aspect outlined by Avison et al. (2007) is the determination of authority that is, "who is really in charge of the research project" (p.28). The authors identified three patterns: client domination, where the research team reports and recommend actions to managers outside the team for their approval; staged domination, which involves passing power on to different stakeholders at various stages of the research; and identity domination, where the researchers are also members of the organisation and thus already have the authority to execute any actions.

The pattern followed in this study was staged domination. I first exerted authority at the Diagnosis Stage for what to consider, how many interviews to carry out, who to talk to, when to stop, etc. In the Planning Stage authority was shared between the Website Task Group and the cluster. Given the fact that the Development Stage took a long time, power domination was distributed between various members of the Task Groups - in particular between the Co-chairs and the cluster Facilitator.

Passing authority to practitioners reduces the researcher's control over the process of the study (Baskerville, 1999; Sommer \& Sommer, 2001). As a researcher, I did not have control over the pace of the Planning and Development Stages. On the other hand, having cluster members as active collaborators gave them a sense of ownership of the project. This ensured that the research participants readily contributed their ideas, talents, and knowledge of local conditions, and advocated for the project. It was important to find a balance to obtain the best of both worlds.

I ensured this balance by subscribing to canonical action research, and therefore the responsibility of data gathering, analysis, and report writing of this study remained with 
me. Therefore I took over for the Evaluation and Specifying Learning Stages, due to my interest in capturing the reflections and learning from this experience.

Finally, Avison et al.'s (2007) third aspect is the degree of formalisation of the power structures, which can range from formal written agreements (e.g. contracts or letters) to informal non-written ones. The authors point out that strictly formal/informal power structures are not common in practice, but rather something in between - according to the circumstances it could progress from one state to the other over time. The degree of formality would depend on: the nature of the researcher, the practitioner organisation, resource provisions, and the problem setting.

This study started with a couple of formal power structures in the form of two letters addressed to the clusters: one scoping my involvement as a volunteer and the second one defining the first stages of the study. Both of them covered most of the points suggested by Baskerville (1999) and Mumford (2001), such as the research domain, objectives, agreed processes, responsibilities, and outputs of the study. Over time, this level of formality was not needed for the clusters, as they were very grateful for any external help they got to assist them in improving their systems.

\subsubsection{Managing expectations}

Before starting the project I discussed with the clusters the nature and potential outcomes of the study, to ensure there were no false expectations about the work that would be done and my role in it. The "action document" proposed by Mumford (2001) and Baskerville (1999) was a written testimony of what was agreed.

Mumford (2001), and West and Stansfield (2001) argue, however, that as the study evolves it may take unforeseen turns which could lead to a change in expectations by those participating in the project. Therefore a degree of flexibility is essential.

Mumford (2001) advocates for open lines of communication, which I constantly maintained with the clusters' Co-chairs and the Facilitator. I reported my progress to them and discussed emerging trends in my study. I also prepared presentations for the rest of the cluster community. For example, on the NHNZ cluster meeting of 26 May 2004, I made a brief presentation to introduce my study, its purpose, my role, and what was the expected level of participation of the cluster. Another example was the presentation I made to EENZ on 17 February 2005, where I briefed the cluster about the results of the Diagnosis Stage. The cluster and I were aware of each other's needs throughout the life of the project and accommodated each other accordingly. 


\subsection{Ethical considerations}

The dual function in action research, of conducting an intervention and generating knowledge, may bring ethical dilemmas to the researcher. The following are some of these dilemmas that have been mentioned in the literature which I encountered in my study.

\subsubsection{Confidentiality}

As Morton (1999) and Mumford (2001) indicated, carrying out action research entails the researcher being more involved with participants and hence they are going to be in close contact with them at various times and settings where some confidential information may be discussed. This raises the ethical issue of the extent to which the researcher should use some of that confidential information in their theory development. I was exposed to several situations where participants made comments outside the cluster or Task Groups meetings that were related to my study. For some of them I decided not to use the information, as I was not prepared to breach the participant's trust. In those cases where I believed the information could add value to the study, I made a mental note to raise the issue again with the same person during an interview session. This way, if the participant felt comfortable sharing it again I was able to capture the relevant information in a formal manner and with their consent.

\subsubsection{Remaining neutral}

As an action researcher I enjoyed a high degree of involvement with the clusters. Nonetheless, I remained neutral regarding their decisions and internal politics. At the time of the research I was a guest in the clusters and did not pass judgment on what was going on. Mumford (2001) warns the action researcher to be cautious and avoid being manipulated into taking sides.

Although no major issues were encountered in this regard during my time with the clusters, I had the support and experience of my two co-supervisors to guide me throughout the inquiry. They provided an objective view of the situations that arose, as they were not immersed in the cluster environment like I was.

\subsubsection{Anonymity}

There were two levels of anonymity considerations in the study: at cluster level and at individual level. I was first inclined not to name the clusters of my study. However, 
given the nature of action research which relies on rich descriptions and the close ties between the subject and its context, it became apparent that people in certain government, academic, and private circles in New Zealand would be able to make an educated guess regarding which clusters I had been working with.

In addition, I was of the view that the clusters and the research would gain more if I could use their actual names. The study could be less ambiguous and gain more credibility, while the clusters could gain recognition of the good work they are doing. Therefore, I approached the clusters and requested their consent to name them, which they kindly granted.

At individual level, on the other hand, participants' anonymity was maintained. Before collecting data from participants - through individual interviews, e-mails, or any other means - I produced letters assuring them their identity would remain anonymous. I also explained to the participants that the data collected were only going to be used for the purposes of this study and would not be released to third parties.

Unfortunately, total anonymity is not going to be possible for some key individuals in the study, due to the fact that they performed specific roles such as the cluster Facilitator, the PWB Liaison Officer, and the Co-chairs. Other cluster members and some stakeholders who work closely with the clusters may be able to identify them. I have informed these participants of this limitation and they have accepted it.

\subsection{Considerations about the credibility of the study}

Checkland and Holwell (2007) argue that knowledge is acquired through an array of processes, ranging from experimental science at one end of the spectrum to story-telling at the other end. For this reason, they propose that the credibility of any knowledge claim should be assessed using criteria that take into consideration the process involved. For example, Checkland and Holwell (2007) mentioned "repeatability" as a credibility criterion for a laboratory experiment, a concept that is not applicable in a qualitative study of a social phenomenon.

Credibility is important if my dissertation is to be accepted by the IS community as a sound study that contributes to knowledge. In this sub-section I focus on detailing the verification steps I took for this study, which have not yet been explicitly discussed. I put forward the credibility criteria that guided my actions: internal validity, recoverability, and generalisability/transferability. 


\subsubsection{Internal validity}

In qualitative studies such as in this one, internal validity is subject to whether the findings and explanations are consistent with the views of the researcher, the participant, or the reader of a particular account (Creswell, 2003; Janesick, 1998). Internal validity was addressed in my study by the following strategies: data triangulation and member-checking, audit trail, and rich descriptions of events.

Data triangulation refers to the use of several independent sources of data to reach a cause from different directions (Creswell, 2003; McNiff, 1988). I collected my data through interviews with various people in charge of different tasks in the cluster(s), newsletter items, meeting minutes and agendas, reports, e-mails, observational notes, my personal diary, and PowerPoint presentations. By considering all these possible sources of data I reduced the risk of bias and incomplete conclusions.

Another important internal validation strategy that I subscribed to was memberchecking. This involved handing over descriptions and findings to participants for them to review and determine if the information was correct (Creswell, 2003). I performed member-checking for various situations in the study. Firstly, transcribed interviews went back to the respective interviewees for verification of their content. Secondly, as a member of the Task Group team that addressed a practical problem, I produced draft documents that were reviewed by the rest of the team. Thirdly, I made interim reports throughout my time with the clusters about findings and themes that I believed were emerging from the data, which I took back to the relevant cluster members for their feedback. And finally, I showed the resulting model to the cluster for them to confirm its accuracy before submitting it for academic scrutiny.

The third internal validation strategy that I pursued was an audit trail, which conveys the careful documentation of the steps taken by the researcher and participants to develop ideas and conclusions (Morse, 1998; West \& Stansfield, 2001). This way, those who are interested in tracking the process back can do so.

Morse (1998) provided a useful list of the types of documentation employed in audit trails which I followed: raw data, data reduction and analysis products, data reconstruction and synthesis products, process notes, material relating to intentions and dispositions, and instrument development information. In addition, West and Stansfield (2001) mentioned that they included diagrams and rough workings of discussions as part of their audit trail. 
Finally, rich descriptions of events and findings was another strategy for validation purposes that I subscribed to. By providing detailed descriptions of the cluster's context and the actions taken during the intervention stages, the reader can gain an understanding of what happened in the study.

\subsubsection{Recoverability}

Checkland and Holwell (2007) proposed "recoverability" as an important criterion for action research studies. That is:

...the aim in AR should be to enact a process based on a declared-inadvance methodology (encompassing a particular framework of ideas) in such a way that the process is recoverable by anyone interested in subjecting the research to critical scrutiny (Checkland \& Holwell, 2007:13).

Throughout this thesis I have stated the underlying philosophical stance of this study; I have proposed a theoretical framework to guide my inquiry; I have given a detailed description of the methodology that I used; I have explained my contributions and role as a researcher; and finally I have discussed how I performed the entry to the site, data collection, data analysis, and exit of the site. By doing so, I hope to provide sufficient evidence for the reader to agree that this study not only can be "recoverable", but can also produce credible findings and conclusions.

\subsubsection{Generalisability/transferability}

Generalisability is another criterion used to assess credibility (Creswell, 2003; Hussey \& Hussey, 1997). This term has to do with applying the results from a study to other situations. Nielsen (2007) prefers the term transferability when referring to this criterion, given the fact that action research studies produce concrete results - not abstract or general ones - that can then be "transferred."

Baskerville and Wood-Harper (1998) and Hussey and Hussey (1997) are of the view that it is possible to generalise some patterns and themes that emerge from a qualitative research to other settings, when the analysis gives sufficient detail of the interactions and characteristics of the situation to help the reader make sense of the situation and be able to relate the findings to other social settings. By providing rich descriptions of the context, the events, and the findings of my study, I have given enough information for the reader to relate this study to other settings with which they are familiar, and then assess how the theory that emerged in my study could be applied in those new settings. 


\section{Chapter 15: Conclusion}

In the introduction I stated my intent of studying how ICT can support knowledge sharing and reuse in clusters. More specifically, I wanted to explore how to improve the knowledge mechanisms that led to successful knowledge transfer for project bidding purposes.

The literature review provided an overview of the business economic environment, and the concepts of cluster, knowledge, and ICT. Firms' business environments are being changed, and many of them acknowledge that they no longer succeed entirely on their own. This study focused on clusters, a "bunching together" of firms, to produce what the markets want or to tackle new opportunities in this business environment.

The literature is still pondering on the role of clusters, with some authors arguing that alternative inter-firm arrangements are as good as clusters in explaining innovation and growth. Regardless of this debate, I believe that clusters play a significant role in the current economic environment and therefore were suitable research subjects to explore how ICT can support them in managing their knowledge-base.

Recent views in the literature see knowledge as a continuum - knowledge has both tacit and explicit dimensions. This is the position I have followed for this study. Similarly, I have subscribed to the notion that information can be viewed as the explicit representation of knowledge. My study has mainly addressed knowledge with a high explicit dimension which, taken outside its context, could be considered as information by some readers.

I particularly explored what the literature has to say about the dynamics of knowledge transfer within a cluster context and how it increases the collective knowledge-base. There was evidence that ICT could play a key role in facilitating this by providing communication, storage, location, and sharing of knowledge functionality.

I have declared that I followed the advocacy/participatory philosophical stance, as stated by Creswell (2003). This was based on the nature of the problem, the research questions, and the way I wanted to address them given my values and beliefs that the phenomena to be studied cannot be detached from their context, and that participants should be given the opportunity to be actively involved in an inquiry that is about them. I also subscribed to the notion that the researcher can get closer to the subjects and contribute towards achieving the solution of the practical problem without such actions 
being viewed as compromising the study. Therefore I used action research methodology to direct my data collection and analysis.

As part of the intervention, the participants and I produced a new portal for one of the clusters. Although our work contributed towards addressing the practical problem for the cluster, it was not the focus of this study. Consequently, expanding knowledge in web-based information systems development or in project management principles was outside the scope of this study. Nevertheless, I reported supplementary findings associated to these areas in Chapter 13 as I believe there was value in examining them from a cluster context.

The following sections cover my concluding statements of the study. I revisit my research questions and related them to my findings. I discuss the findings of this study in the context of the literature review. I then state my contributions to knowledge. Based on this experience, I also offer my take on the implications for practice and directions for future research. The final section is an update of what the clusters and key players have been doing since my exit as a researcher; attention is paid to what the cluster has done with its new portal.

\subsection{Revisiting the research questions}

The two research questions this study has embarked on answering were:

1) What are the knowledge transfer mechanisms in a cluster when it performs a business activity such as international project bidding?

2) How can ICT be used to improve cluster knowledge transfer during project bidding?

The subjects of my study were Earthquake Engineering NZ (EENZ) and Natural Hazards NZ (NHNZ), two clusters based in Wellington, New Zealand. These are matured clusters: EENZ was formed nearly 10 years ago, while NHNH is over eight years old. The clusters are actively pursuing their goals; they have strong track records and have been successful in winning international projects.

Both clusters count on the support of their regional development agency. The clusters have some cross-pollination due to facts that they share the same Facilitator and that key companies have joined both clusters. In addition, these clusters constantly accessed 
their internal and external relationships for new knowledge in order to maintain their innovation and performance over time.

For this study, I departed from the premise that genres are knowledge transfer mechanisms. In Chapter 3 - specifically Section 3.5 - I made the association between the genre and the knowledge management literature about these two concepts. Consequently, the concept of genre was the analytical unit that helped me answer my research questions.

My first question was answered in the Diagnosis Stage of the study. To do so I first mapped the clusters' bidding process. While working on this, the participants and I recognised that, in fact, project bidding may follow two approaches: one where consultants reactively respond to a procurement notice, and the other where the clusters take the initiative of approaching a client to discuss a potential project. The outcomes were two diagrams (Figures 17 and 18) that illustrated the steps taken in each approach.

I then applied Genre Theory - more specifically Genre Systems - to identify the collaborative interactions (i.e. genres) for each step in both project bidding approaches. These collaborative interactions are precisely the clusters' knowledge transfer mechanisms that I set myself to find.

Genre Systems looked at each collaborative interaction in terms of purpose, content, actors, form, time, and place. The result was a "Project Bidding" system of coordinated and distinctive genres whose primary purpose was to win a contract. In the case of the clusters, a large variety of genres are exchanged and invoked many times in "Project Bidding." The complete list of genres in this system can be found in Appendix E. Answering the first research question was a straightforward but time-consuming exercise.

From the level of detail that Genre Systems demanded when analysing each genre, I inferred that those genres with high tacit content have been well served through conventional media such as phone calls and face-to-face meetings. However, those genres with a high content of explicit knowledge could be more effectively managed by having a centralised "space" - something that ICT can provide.

Genre Systems Analysis also allowed me to observe that, in five sub-systems of Project Bidding, not only was a large variety of genres invoked, but their traffic was also high: Searching and Tracking new Opportunities, Preparing EOIs, Preparing Tender 
(reactive) or Proposal (proactive), and Building Relationships. These sub-systems have the potential to benefit from an ICT solution.

The second research question was more complex to tackle. Being a "how" question, there were a number of alternatives for ICT to improve the clusters' knowledge transfer mechanisms. The final decision on the areas of ICT to explore further rested with the clusters.

At the time, NHNZ was the only cluster ready to engage in the next stages of action research, and implemented an ICT solution to improve its knowledge transfer. The cluster opted to revamp its website to follow the portal model, and to incorporate a database to manage its content and documents. I volunteered to be part of its Task Groups. The job encompassed working with the Task Groups, taking into account the limited resources and time available for those involved.

The revamped NHNZ website introduced a tool to assist in the management of the cluster's knowledge-base. It provided a space to share and store some of the genres associated with the Project Bidding system. To make the most of this new medium, three commonly used genres were "digitised": the Expert Profile, the Company Profile, and Project Sheet. The database and Internet enabled these genres to be searchable, easy to update, accessible anywhere and anytime, and even (in the case of Company Profile) to have hyperlinks. This was how ICT played a role in improving the clusters' knowledge transfer mechanisms during my study.

The study showed how ICT can play a fundamental role in facilitating knowledge transfer in a business cluster by providing fast, reliable, and cheap communication channels, and by allowing cluster members to store and access resources and information to accomplish specific tasks.

\subsection{Discussion of findings}

The literature on knowledge transfer has focused on identifying the elements and conditions needed for successful knowledge transfer, such as the models proposed by Easterby-Smith et al. (2008), Minbaeva (2007), and Rolland and Chauvel (2000). All of them have identified the need for knowledge to be "articulated" or "packed" to make the transfer possible. I called these "packages" a knowledge transfer mechanism. The literature confirmed that this aspect was worth exploring further. 
I proposed that genres are knowledge transfer mechanisms. This allowed me to tap into the literature on genres to expand on what has already been put forward on knowledge transfer mechanisms. For instance, the typologies put forward by Jasimuddin (2007) and Smeds et al. (2001) can be enriched by considering the purpose, content, actors, form, time, and place of the knowledge transfer mechanisms - something that genre analysis already does. By doing so, other variables that also influence knowledge transfer can be considered when assessing the appropriateness of a mechanism. This study, however, went further than merely mapping and assessing the knowledge transfer mechanisms using Genre Systems approach.

In genre and knowledge transfer literature there have been references to a transformative process. The work by Shepherd and Watters (1998) and Ihlström and Henfridsson (2005) in Genre Theory proposes that genres experience a progression when they are taken from the physical world to the electronic one. Similarly, in the knowledge camp, Guzman and Trivelato (2008) refer to a three-stage process for knowledge transfer (knowledge codification, mechanisms used to transfer knowledge, and knowledge assimilation and application). I parted from these views to explore the process of how knowledge transfer mechanisms are digitised to take advantage of the functionality of the new electronic medium that the Internet and other technologies can offer. The outcome was a five-step model that explains the process of creating digital genres.

Going through the exercise of developing an ICT application for a cluster provided the opportunity to explore the steps undertaken for some of the cluster's genres in order to be incorporated into the new system. As Ihlström and Henfridsson (2005) pointed out, such progression does not emerge in a social vacuum. In addition to the coding work required by the developer to create the templates and online forms for the chosen genres, and the data entry work carried out by the participants, the cluster needed to establish social rules around the content of these genres, such as who could view and edit them, and how they should be used. The cluster, as a social group, needed to legitimise the digital genres as the right ones for the particular job.

As a result of the intervention, the clusters' knowledge-base in project bidding was mapped during the Diagnosis Stage; and for one of them it was increased through the experience of developing a portal in the Planning and Development Stages. Tallman et al.'s (2004) framework on the types of knowledge that are being shared within a cluster was a good starting point for this study to explain how the clusters increased their 
knowledge-bases on project bidding. The concept of component and architectural knowledge - which I applied to the genres - allowed me to analyse the clusters' dynamics of knowledge flow and how ICT could support them.

From this analysis, I proposed that a cluster's knowledge-base grows in natural and induced ways. Natural ways occur when the cluster interacts while pursuing bids opportunities, and therefore the cluster left alone is capable of increasing its knowledgebase over time. Induced ways occur when the cluster proactively develops knowledge to change its status quo. This was the case when the Task Groups and I developed NHNZ's Portal.

We brought together existing component knowledge from some of the Task Group participants. For example, the cluster Facilitator provided some of the templates that the cluster had already used in previous EOIs and tenders as a starting point, and a Task Group member provided the templates to be studied from their personal data repositories to prepare the one-page Project Sheet genre. These contributions brought new knowledge to the cluster and avoided "reinventing the wheel."

By planning and developing the Portal, we increased the NHNZ cluster's architectural knowledge on website development and project management - in particular, those cluster members involved in the Task Groups. The cluster also increased its architectural knowledge when it defined the social rules around the digital genres that were created as part of the Portal development.

The development of a Portal - with the potential to become the cluster's knowledge repository - and digital genres as mechanisms to transfer some of this knowledge gave the opportunity for NHNZ to have their knowledge available in a form that is easy to share and reuse amongst members. As Haefliger et al. (2008) pointed out, success in knowledge sharing and reuse depends more on organisational factors than on technical ones. In this case, the cluster was committed to developing the tools and rules to enable reuse, and the Co-chairs championed - and partly funded - the project to make it happen. This study confirmed the conditions that Molina and Yoong (2003) outlined for favouring knowledge sharing, such as having a common understanding and commitment to the cluster idea, a high level of trust, and strong leadership.

EENZ and NHNZ initiatives have been developed under a set of conditions that would mould the clusters' knowledge-base (depicted in Figure 24). The small budget they were able to allocate tended to limit their breadth. This was compounded by the fact that 
cluster members who volunteered time to work on such initiatives always prioritised their individual work over the cluster, and thus slowed progress down. Furthermore, members' level of participation and up-take depended on how relevant the new knowledge was for their individual company. On the other hand, using a project coordinator, the support of champions, and the local economic development agency, and setting up an event as a deadline, have been identified as conditions that motivate the cluster to move ahead in completing an initiative.

It is therefore no surprise to see that, in order to succeed under these circumstances, many initiatives to enhance the clusters' knowledge-base were done as gradual progressions over a long period of time.

\subsection{Contributions to knowledge}

This study has contributed to the enhancement of knowledge on various fronts. Firstly, by proposing that genres are knowledge transfer mechanisms, I have considered new ways to identify and analyse knowledge transfer mechanisms. Genre Systems can be used as a diagnostic tool to understand the communicative interactions of a business process, such as project bidding, and thus shed light on the appropriateness of each of its transfer mechanisms. The richness of Genre Systems Analysis allowed the identification of what sub-systems and which genres to consider for further assessment in terms of knowledge management.

The second contribution of this study was to explore the evolution of some genres invoked by the cluster during project bidding into digital ones as a result of introducing ICT. The arrival of faster and powerful computing capacity, combined with revolutionary applications such as the Internet and the World Wide Web, provided a new communication medium where these digital genres are hosted.

The proposed five-step digitisation model revealed that digitising genres entail more than converting text into bytes. There are social rules, testing, and validation around these genres before they become appropriate knowledge transfer mechanisms.

The study has brought together genre and knowledge transfer concepts to a new business context, which is the cluster environment. By studying several related genres, a more holistic view of the clusters being part of this study, their social actions, and communication practices was reached. 
Most of the literature on business clusters has an economic perspective, which discusses the effectiveness of this approach for regional and national development. There are few studies focused on the cluster's knowledge-base and its management, and even fewer that look at the role of ICT in supporting knowledge transfer in a cluster environment. This study has contributed to the current stream of research on business clusters by considering these two aspects.

Clusters have the potential to generate knowledge to satisfy the needs of Markus's (2001) types of knowledge re-users. The lessons learnt and documentation generated in each project bid that the cluster engaged with could be shared and reused to either improve the quality or speed-up the cluster's business processes, products, and services. The study proposed ICT as a means to ensure knowledge sharing and re-use.

The new NHNZ portal played various key roles. Firstly, it facilitated knowledge by capturing, packaging, distributing and reusing the knowledge already held in the cluster. It also triggered the creation of new component knowledge, such as the one-page Project Sheets and Company Profiles. Finally, the experience of planning and developing the Portal increased the NHNZ cluster's architectural knowledge on ICT and how it can be employed to provide better solutions.

A fourth contribution of this study was identifying the factors that clusters have to consider when developing initiatives to enhance their knowledge-base. Being entwined with the context, the Task Groups and I experienced the influence that these factors have on the growth of the cluster's knowledge-base. Some of the challenges that emerged were in accordance to the literature of project development (i.e. budget, supportive champions, and user up-take). However, new considerations and themes emerged due to the context of a cluster, such as commitment of volunteers, support of an economic development agency, and a trigger event.

Finally, this study contributed to the IS discipline by providing an example where action research was employed to enhance knowledge. This enriches the knowledge base in IS with the findings of a study that did not follow the traditional quantitative/positivist/post positivist view.

In several instances during my study I wondered if action research was the right methodology to address my research questions. I believe that other methodologies, such as case study, could have also been suitable for this enquiry. Nonetheless, the richness of the descriptions, being involved with the cluster for a long time, and being allowed to 
participate as a team member, enabled me to get good insights into how the clusters operate, their context, and what factors influenced their knowledge-bases.

\subsection{Implications for practice}

By taking part in the study, the participating clusters gained better understanding of their own project bidding processes and how ICT can support them. This in turn would help clusters to develop future strategies and consider other ICT systems to improve their knowledge transfer mechanisms in project bidding.

Apart from addressing the practical problem, the clusters have a written account of the situation and how we went on to solve it, thus increasing the clusters' organisational memory. This knowledge will be valuable if the clusters decided to develop digital genres for other of their key steps in the Project Bidding System. Furthermore, there is potential to explore other business cluster processes such as marketing, product development, or applying for funding, as they were outside the scope of this study.

Through the exercise of developing a portal, NHNZ in particular increased its architectural knowledge on systems development, and how ICT can be incorporated to provide better solution for their project bidding needs. In addition, they became aware of the issues and challenges to tackle when engaging in a deliberate act to organise their knowledge-base.

By having a more organised knowledge-base for their project bidding, the knowledge creation process is more focused, as gaps in knowledge are identified. For example, the NHNZ cluster noticed the need to develop case studies (which are more in-depth descriptions) of key projects carried out by members. These summaries were made available through the Portal.

The lessons learnt throughout this study could be "cross-fertilised" to other clusters in the region, as already suggested by one participant:

And that's one of the good things about making all this documenting and writing up. What are the learnings from it all? I think there is a brilliant case study here for others. I hope you are going to do some good presentations of case studies or some seminars about this. (Interview Task Group member, 06 December 2005). 


\subsection{Directions for future research}

The study showed how Genre Systems can assist business clusters in identifying the nature of their collaborative interactions within a set of key clusters' activities and provide a mapping of these activities to assist where and how ICT could be deployed to facilitate better knowledge sharing and re-use. Future research could explore how Genre Systems be applied to other collaborative groups such as business networks and communities of practice.

The combination of computing and the Internet have created a new medium where digital genres are flourishing. This study just explored one aspect of this frontier by proposing a five-step process that explains how old genres are being digitised to exist in this medium; there is still more ground to cover in this domain where new genres are emerging with no equivalent in other media.

The five-step process of digitising genres was developed based on our experience working with three genres in the NHNZ cluster. Like the case of Genre Systems, this framework should be studied further to verify it can explain how groups in other contexts take their known genres into the digital medium.

More studies should be carried out to gain further insights on the role of ICT in the dynamics of knowledge transfer in clusters. Researchers may be interested in analysing the use of technology by this type of collaborative group. For instance, this study focused on the transfer of highly explicit knowledge, leaving room for exploring how ICT could support clusters in managing their tacit knowledge. Furthermore, it would be particularly useful to find ways to improve the level of uptake of new technologies by the cluster members, given the challenges described in Chapter 13.

Finally, I would encourage researchers in the IS discipline to consider action research in their enquiry. The IS knowledge-base will be enriched with the findings of studies that do not follow the traditional quantitative/positivist/postpositivist view.

\subsection{Update}

Natural Hazards New Zealand and Earthquake Engineering New Zealand continue operating in the Wellington region. Both clusters have been successful vehicles to tackle international opportunities and this was reflected by becoming finalists for the 2007 Wellington Region Gold Awards, which is a highly regarded business award for enterprise and excellence. 
Although I stopped collecting data and exited the site in December 2005 to engage in theory development and write-up of the final report for the research component of the study, I maintained a relationship with the clusters. As a result of this experience I was offered employment at one of the organisations that is a member of both clusters. One of my tasks in my new job is to provide website administration support to the NHNZ portal. This way the cluster has guaranteed some continuity on the work we started back in 2004.

The cluster has shown commitment to developing and maintaining their knowledgebase infrastructure further by including in its budget 10 hours of IT development a year, which they managed to negotiate with the hosting organisation. The amount sounds small, but considering the limited resources NHNZ has available to perform its activities, it is sending the right signal that the cluster is prepared to carry out ongoing IT development.

Since the website launch in November 2005, NHNZ has engaged in three development rounds for the portal, none of which were of the scale of the cycle described in this thesis. The first one took place in early 2006 , and created the data share functionality in the private section of the portal for the cluster to store their documents and resources: minutes, agendas, marketing material, old proposals, genre templates, briefing papers, and the cluster incorporated society rules. NHNZ now counts on a repository for their written knowledge and organisational memory for reference and future reuse.

The second development round took place in mid 2007, when the cluster received a small grant from Positively Wellington Business before it was disestablished as the region's development agency. This grant allowed the review of the portal's content and a redesign of its look and feel. Taken into account the learning from this study's intervention, a small team comprised of the NHNZ executive and I worked closely with a copywriter to create content for many of the portal's pages. This cluster team also engaged with a design company to deliver the specifications for a new design of the portal with additional functionality to make the site appealing to potential clients, including showcasing an expert and a feature project every time a user visits the website.

A third round started in early 2008, when funding was secured by one of the cluster members to develop the proposed designs for the new look and functionality of the portal. In addition, the administration system for the "Interesting Links" - which couldn't be included during the intervention of this study - finally got developed for the 
Portal. Once again, the same challenges outlined in Chapter 14 were faced by the small team, but progress was steady and the new portal went live in August 2008. Figure 25 is a snapshot of the current's NHNZ Homepage.

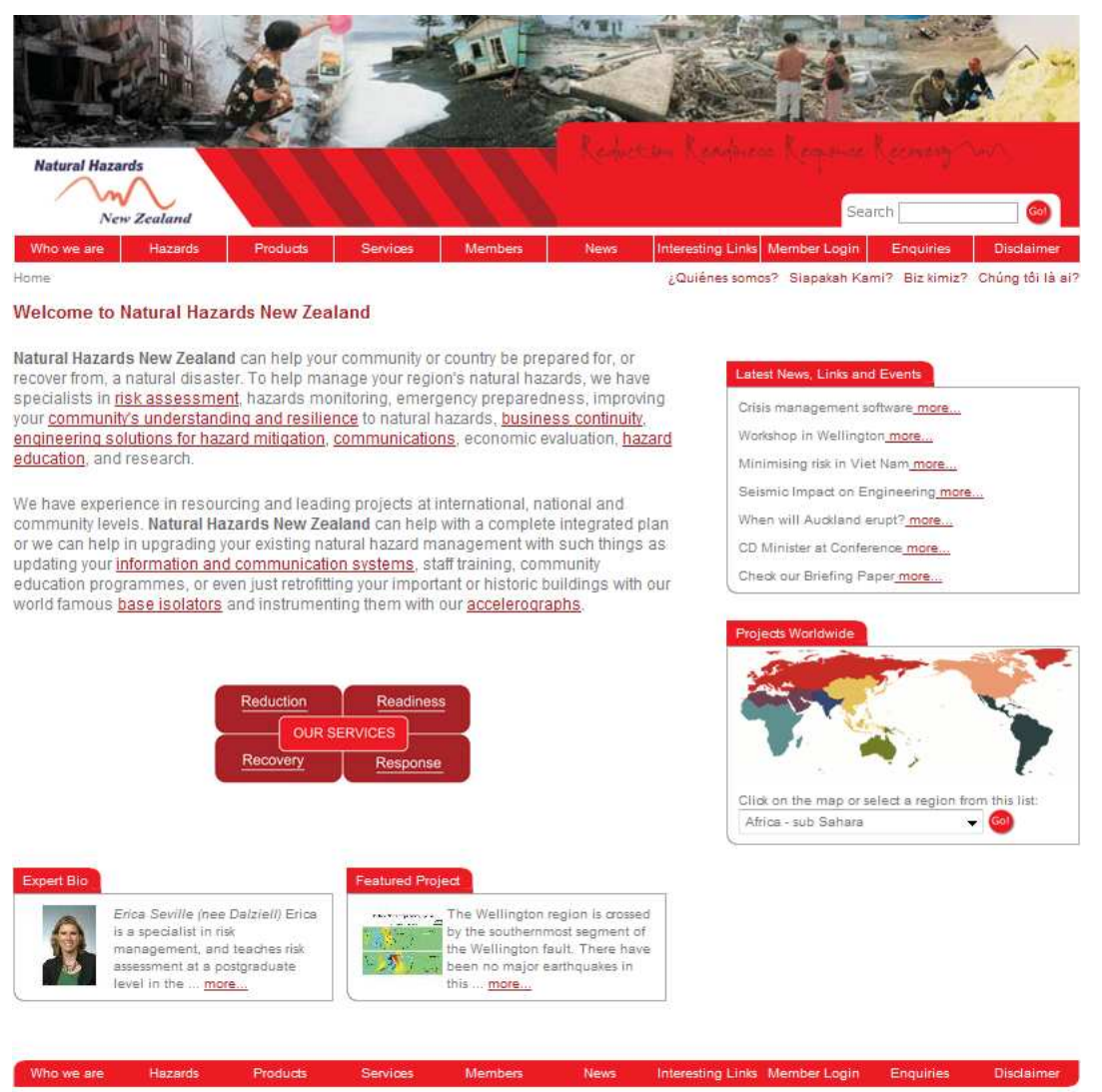

Figure 25: Current NHNZ Portal's Homepage. (Source: www.naturalhazards.co.nz)

With regards to the three genres that were developed during the study, they remain the same, although the look of the Project Sheet has changed as part of the third round of development. The use of the portal as a project bidding tool by cluster members remain low, something that the NHNZ Executive would like to address.

Grow Wellington is the new region's development agency. This agency is working closely with both EENZ and NHNZ clusters, as one of its objectives is to develop a world-leading Centre of Excellence in the area of natural hazards by 2010 . 


\section{References}

Agarwal, R. \& Venkatesh, V. (2002). Assessing a firm's web presence: A heuristic evaluation procedure for the measurement of usability. Information Systems Research, 13(2), 168-186.

Alavi, M. \& Leidner, D. E. (2001). Knowledge management and knowledge management systems: Conceptual foundations and research issues. MIS Quarterly, 25(1), 107-136.

Albino, V., Garavelli, A.C. \& Gorgoglione, M. (2004). Organization and technology in knowledge transfer. Benchmarking, 11(6), 584-600.

Albino, V., Garavelli, A.C. \& Schiuma, G. (1999). Knowledge transfer and interfirm relationships in industrial districts: The role of the leader firm. Technovation, 19(1), 53-63.

Alsleben, C. (2005). The downside of knowledge spillovers: An explanation for the dispersion of high-tech industries. Journal of Economics, 84(3), 217-248.

Altrichter, H., Kemmis, S., McTaggart, R. \& Zuber-Skerrit, O. (2002). The concept of action research. The Learning Organistion, 9(3/4), 125-131.

Alvesson, M. \& Kärreman, D. (2001) Odd couple: making sense of the curious concept of knowledge management. Journal of Management Studies, 38(7), 997-1018.

Anderson, E.S., Birchall, D., Jessen, S.A. \& Money A.H. (2006). Exploring project success. Baltic Journal of Management, 1(2), 127-147.

Appleyard, M. M. (1996). How does knowledge flow? Interfirm patterns in the semiconductor industry. Strategic Management Journal, 17(Special Issue), 137-154.

Argote, L. and Ingram, P. (2000). Knowledge transfer in organizations: Learning from the experience of others. Organizational Behaviour and Human Decision Processes, 82(1), 150-169.

Asheim, B.T. \& Isaksen, A. (2002). Regional innovation systems: The integration of local 'sticky' and global 'ubiquitous' knowledge. Journal of Technology Transfer, 27(1), 77-86.

Askehave, I. \& Nielsen A.E. (2005). Digital genres: A challenge to traditional genre theory. Information Technology \& People, 18(2), 120-141. 
Avison, D., Baskerville, R. \& Myers, M. (2001). Controlling action research projects. Information Technology \& People, 14(1), 28-45.

Avison, D., Baskerville, R. \& Myers, M. (2001). The structure of power in action research projects. Information Technology \& People, 14(1), 28-45.

Avison, D., Baskerville, R. \& Myers, M. (2007). The structure of power in action research projects. In Kock, N. (Ed.), Information Systems Action Research (pp.19-41). New York: Springer Science.

Avison, D., Lau, F., Myers, M. \& Nielsen, P. A. (1999). Action research. Communications of the ACM, 42(1), 94-97.

Bailey R., Koziarski, A. \& Knight, L. (August, 2002). Evaluation of the cluster development pilot. Wellington, New Zealand: Industry New Zealand. Available at: http://www.industrynz.gvt.nz/redion/_documents/evaluationclusterdevelopmentpilot.pdf . [Date accessed: April 25, 2003].

Baskerville, R. (1999). Investigating information systems with action research. Communication of the AIS, 2(19), 1-23.

Baskerville, R. (2001). Conducting action research: High risk and high reward in theory and practice. In Trauth, E.M (Ed.), Qualitative research in IS: Issues and trends (pp.192-217). Hershey, PA, USA: Idea Group Publishing.

Baskerville, R. \& Pries-Heje, J. (1999). Grounded action research: A method for understanding IT in practice. Accounding Management \& Information Technology, 9(1), 1-23.

Baskerville, R. \& Stage, J.. (1996). Controlling prototype development through risk analysis. MIS Quarterly, 20(4), 481-504.

Baskerville, R. \& Wood-Harper, A. T. (1996). A critical perspective on action research as a method for information systems research. Journal of Information Technology, 11(3), 235-246.

Baskerville, R. \& Wood-Harper, A. T. (1998). Diversity in information systems action research methods. European Journal of Information Systems, 7(2), 90-107.

Bawarshi, A. (2000). The Genre function. College English, 62(3), 335-360. 
Bazerman, C. (1994). Systems of genres and the enactment of social intentions. In Freedman, EA \& Medway, P. (Eds.), Genre and the New Rhetoric (pp.79-101). London: Taylor \& Francis.

Bazerman, M. (1994). Judgement in managerial decision making $\left(6^{\text {th }}\right.$ ed.). New York: J. Wiley

Bell, G.G. (2005). Clusters, networks, and firm innovativeness. Strategic Management Journal, 26(3), 287-295.

Bell, D.G., Giordano, R., \& Putz, P. (2002). Inter-firm sharing of process knowledge: Exploring knowledge markets. Knowledge and Process Management, 9(1), $12-22$.

Benbasat, I., Goldstein, D. \& Mead, M. (1987). The case research strategy in studies of information systems. MIS Quarterly, 11(3), 369-386.

Bentley, T. (1998). Information systems strategy for business. London: CIMA.

Benville, C. \& Landry, M. (1992). Can the field of MIS be disciplined? In Galliers, R. (Ed.), Information systems research: Issues, methods and practical guidelines (pp.61-88). Oxford: Blackwell Scientific Publications.

Bergman, E. \& Feser, E. (1999). Industry clusters: A methodology and framework for regional development policy in the United States. In OECD proceedings boosting innovation:The cluster approach (pp. 243-268). Paris: OECD.

Bergquist, M. \& Ljungberg, J. (1999). Genres in action: negotiating genres in practice. In Proceedings of the 32nd Hawaii International Conference on System Sciences, 5-8 January 1999 (pp. 11). Los Alamitos: IEEE Press.

Bergquist, W., Betwee, J. \& Meuel, D. (1995). Building strategic relationships. San Francisco, USA: Jossey-Bass Publishers.

Berranger, P. \& Meldrum, M.C. (2000). The development of intelligent local clusters to increase global competitiveness and local cohesion: The case of small business in the creative industries. Urban Studies, 37(10), 1827-1835.

Best, M. (1990). The new competition: Institutions of industrial restructuring. Cambridge: Harvard University Press. 
Bhatt, G. (2001). Knowledge management in organizations: Examining the interaction between technologies, techniques, and people. Journal of Knowledge Management, 5(1), 68-75.

Brandt-Husmann, T. (2001) Inter-organistional learning and knowledge transfer: A taxonomy of knowledge transfer costs. In DRUID (Danish Research Unit for Industrial Dynamics) Winter Conference. Klarskovgaard, Denmark. Available at: http://www.druid.dk/conferences/winter2001/paper-winter/Paper/tina1.pdf [Date accessed: May 17, 2004].

Braun, P. (2003a). .comUnity: A study on the adoption and difussion of Internet technologies in a regional tourism network. Unpublished doctoral dissertation, University of Ballarat, Victoria, Australia.

Braun, P. (2003b). Regional tourims networks: The nexus between ICT diffusion and change in Australia. Information Technology \& Tourism, 6(4), 231-243.

Braun, B., Gaebe, W., Grotz, R., Okamoto, Y. \& Yamamoto, K. (2002). Regional networking of small and medium-sized enterprises in Japan and Germany evidence from a comparative study. Environment and Planning A, 34(1), 81-99.

Brimblecombe, P. (1999). The Wellington business clusters project: A case study. Unpublished master's thesis, Victoria University of Wellington, Wellington.

Brioschi, F., Brioschi, M. \& Cainelli, G. (2002). From the industrial district to the district group: An insight into the evolution of local capitalism in Italy. Regional Studies, 36(9), 1037-1052.

Bryant, J. (2003). The six dilemmas of collaboration: Inter-organisational relationships ad drama. West Sussex, England: John Wiley \& Sons, Ltd.

Burns, A. (1999). Collaborative action research for English language teachers. Cambridge: Cambridge University Press.

Cainelli, G. \& De Liso, N. (2005). Innovation in industrial districts: Evidence from Italy. Industry and Innovation, 12(3), 383-398.

Carbonara, N. (2005). Information and communication technology and geographical clusters: opportunities and spread. Technovation, 25(3), 213-222).

Carroll, G. (2006). Earthquake Engineering NZ business cluster leveraging benefits and opportunities, a facilitator's perspective. In Proceeding of the 2006 NZSEE Conference, 10-12 March 2006 (paper No.43). Napier, New Zealand:NZSEE 
Ceglie, G., Clara, M. \& Dini, M. (1999). Cluster and networks development projects in developing countries: Lessons learned through the UNIDO experience. In OECD proceedings boosting innovation: The cluster approach (pp. 269-289). Paris: OECD.

Chaminade, C. (1999). Innovation processes and knowledge flow in the information and communications technologies (ICT) clusters in Spain. In $O E C D$ proceedings boosting innovation: The cluster approach (pp. 219-242). Paris: OECD.

Checkland, P. (1991). From framework through experience to learning: The essential nature of action research. In Nissen, H., Klein, H. \& Hirschheim, R. (Eds.), Information systems research: contemporary approaches \& emergent traditions (pp.397-403). Amsterdam, the Netherlands: Elsevier Science Publishers.

Checkland, P. \& Holwell, S. (2007). Action research: Its nature and validity. In Kock, N. (Ed.), Information systems action research (pp. 3-17). New York: Springer Science.

Chiasson, M. \& Dexter, A.S. (2001). System development conflict during the use of an information systems prototyping method of action research. Information Technology \& People, 14(1), 91-108.

Chiesa, V. \& Chiaroni, D. (2005). Industrial clusters in biotechnology - driving forces, development processes and management practices. London: Imperial College Press.

Chisholm, R.F. \& Elden, M. (1993). Features of emerging action research. Human Relations, 46(2), 275-298.

Ciborra, C.U. \& Andreu, R. (2001). Sharing knowledge across boundaries. Journal of Information Technology 16(2), 73-81.

Cleland D.I. \& Ireland, L.R. (2006). Project management: Strategic design and implementation. ( $5^{\text {th }}$ ed.). New York: McGraw-Hill.

Coakes, E. (2006). Storing and sharing knowledge: Supporting the management of knowledge made explicit in transnational organisations. The Learning Organization, 13(6), 579-593.

Cockburn-Wootten, C., Henderson, A. \& Rix, C. (2005). Learning from the apprentice: an account of action research practice in a university department. Action Learning: Research and Practice, 2(1), 71-78. 
Connelly, C. \& Kelloway, E. (2003). Predictors of employees' perceptions of knowledge sharing cultures. Leadership \& Organization, 24(5), 294-301.

Creswell, J.W. (1994). Research design: Qualitative \& quantitative approaches. Thousand Oaks, California, USA: SAGE Publications Inc.

Creswell, J.W. (2003). Research design: Qualitative, quantitative and mixed methods approaches ( $2^{\text {nd }}$ ed.). Thousand Oaks, California, USA: SAGE Publications Inc.

Crocombe, G., Enright, M. \& Porter, M. (1991). Upgrading New Zealand's competitive advantage. Auckland: Oxford University Press.

Crowston, K. \& Williams, M. (2000). Reproduced and emergent genres of communication on the World Wide Web. The Information Society, 16(3), 201-215.

Cuckier, W., Cody, S. \& Nesselroth, E. (2006). Genres of spam: Expectations and deceptions. In Proceedings of the 39th Hawaii International Conference on System Sciences, 4-7 January 2006 (pp. 9). Los Alamitos: IEEE Press.

Dahl, M.S. \& C. Ø. Pedersen (2004). Knowledge flows through informal contacts in industrial clusters: myth or reality? Research Policy, 33(10), 1673-1686.

Davenport, T. \& Prusak, L. (1998). Working knowledge: How organizations manage what they know. Boston: Harvard Business School Press.

Davison, R. (2001). GSS and action research in the Hong Kong police. Information Technology \& People, 14(1), 60-77.

Davison, R., Martinsons, M. \& Kock, N. (2004). Principles of canonical action research. Information Systems Journal, 14(1), 65-86.

Dearlove, D. (2001). The cluster effect: Can Europe clone Silicon Valley? Strategy \& Business [On-line serial] (third quarter), 24: 1-10. Available at http://www.strategy-business.com/press/article/?art=14900\&pg=0 [Date accessed: April 24, 2003].

de Berranger, P. \& Meldrum, M. (2000). The development of intelligent local clusters to increase global competitiveness and local cohesion: The case of small business in the creative industries. Urban Studies, 37(10), 1827-1836.

DeFillippi, R.J. (2002) Organizational models for collaboration in the new economy. Human Resource Planning, 25(4), 7-18. 
Detlor, B. (2000). The corporate portal as information infrastructure: towards a framework for portal design. International Journal of Information Management, 20(2), 91-101.

DeLuca, D., Gallivan, M. \& Kock, N. (2008). Furthering information systems action research: A post-positivist synthesis of four dialectics. Journal of the Association for Information Systems, 9(2), 48-72.

Denzin, N.K. \& Lincoln, Y.S. (1998). Entering the field of qualitative research. In Denzin, N.K. \& Lincoln, Y.S. (Eds.), Strategies of qualitative inquiry (pp.56-85). Thousand Oaks, CA, USA: SAGE Publications, Inc.

Devlin, K. (2001) InfoSense: Turning information into knowledge. New York: W.H. Freeman and Company.

Dias, C. (2001). Corporate portals: A literature review of a new concept in information management. International Journal of Information Management, 21(4), 269-287.

Dickens, L. \& Watkins, K. (1999). Action research: Rethinking Lewin. Management Learning, 30(2), 127-140.

Drucker, P. (1994). The age of social transformation. The Atlantic Monthly, 274(5), 53-80.

Drucker, P. (1998). The coming of the new organisation. In Harvard Business Review on knowledge management (pp. 1-20). Boston: Harvard Business Review.

EENZ \& NHNZ. (2007). Global Gold Awards Entry. Internal Document.

El Sawy, O.A., Malhotra, A., Gosain, S. \& Young, K. M. (1999). IT-intensive value innovation in the electronic economy: Insights from Marshal Industries. MIS Quareterly, 23(3), 305-335.

Elden, M. \& Chisholm, R.F. (1993). Emerging varieties of action research: Introduction to the special issue. Human Relations, 46(2), 121-142.

Enright, M. J. \& Roberts, B. H. (2001). Regional clustering in Australia. Australian Journal of Management, 26(Special Issue), 65-85. Available at http://www.agsm.unsw.edu.au/eajm/current.html [Date accessed: April 5, 2002]. 
Ferrán-Urdaneta, C. \& Storck, J. (1997). Truth or deception: the impact of videoconferencing for job interviews. In Kumar, K. \& DeGross, J. (Eds.), Proceedings of the eighteen international conference on information systems, 15-17 December 1997 (pp. 183-196). Georgia, USA: ICIS.

Ffowcs-Williams, I. (1996). Hard and soft networks: Helping firms co-operate for export growth. New Zealand Strategic Management, 2(2), 30-36.

Ffowcs-Williams, I. (1997). Local clusters and local export growth. New Zealand Strategic Management, 2(4), 24-30.

Firth, D. \& Lawerence, C. (2003). Genre analysis in information systems research. Journal of Information Technology Theory and Application, 5(3), 63-77.

Franke, U. \& Hickmann, B. (1999). Is the net-broker an entrepreneur? What role does the net-broker play in virtual Webs and virtual corporations? Electronic Journal of Organizational Virtualness, 1(1), 120-138. Available at: http://www.virtualorganization.net [Date accessed: March 30, 2004].

Freedman, A. \& Medway, P. (1994). Locating genre studies: Antecedents and prospects. In Freedman, A. \& Medway, P. (Eds.), Genre and the New Rhetoric (pp.120). London: Taylor \& Francis.

French, W.L. \& Bell, C. H. (1999). Organization development: Behavioural science interventions for organization improvement ( $6^{\text {th }}$ ed.). Upper Saddle River, New Jersey, USA: Prentice Hall.

Gallant, L., Irizarry, C., Kreps, G. (2007). User-centric hospital web sites: A case for trust and personalization. E-Service Journal, 5(2), 5-26.

Galliers, R. (1991). Choosing appropriate information systems research approaches: A revised taxonomy. In Nissen, H., Klein, H.K. \& Hirschheim, R. (Eds.), Information systems research: Contemporary approaches \& emergent traditions (pp.327-345). Amsterdam, The Netherlands: Elsevier Science Publishers.

Garceau, L.R. (1990). Documentation and training as a systems development tool. The CPA Journal, 60(11), 84-89.

García, L., \& Quek, F. (1997). Qualitative research in information systems: Time to be subjective? In Lee, A.S, Liebenau, J. \& DeGross, J.I. (Eds.), Information systems and qualitative research (pp.444-465). London: Chapman \& Hall.

George, Carole. (2005). Usability testing and design of a library website: An iterative approach. OCLC Systems and Services, 21(3), 167-180. 
Ghasemzadeh, F. \& Archer N.P. (2000). Project portfolio selection through decision support. Decision Support Systems 29(1), 73-88.

Gittos, A., van Opdorp, C.. \& Gray, C. (April, 2005). 2004 Collaborative cluster and networks profile. Wellington, New Zealand: Positively Wellington Business.

Giuliani, E. (2007). The selective nature of knowledge networks in clusters: Evidence from the wine industry. Journal of Economic Geography 7(2), 139-168.

Glaser, B.G. \& Strauss, A.L. (1967). The Discovery of grounded theory: Strategies for qualitative research. Chicago, USA: Aldine Publication Co.

Gordon, I. R. \& McCann, P. (2000). Industrial clusters: Complexes, agglomeration and/or social networks? Urban Studies, 37(3), 513-532.

Gordon, I. R. \& McCann, P. (2005). Innovation, agglomeration, and regional development. Journal of Economic Geography, 5(5), 523-543.

Gray, C., Harvey, O. \& Brimblecombe, P. (November, 2001). Cluster classification and costing research. Report commissioned by Industry New Zealand, New Zealand. 317.

Gulati, R. (1998). Alliances and networks. Strategic Management Journal 19, 293 -

Guzman, G. \& Trivelato, L.F. (2008). Transferring codified knowledge: sociotechnical versus top-down approaches. The Learning Organization, 15(3), 251-276.

Haag, S., Cummings, M. \& McCubbrey, D. (2005). Management information systems for the information age. ( $5^{\text {th }}$ ed.). McGraw-Hill/Irwin: New York.

Haefliger, S., von Krogh, G. \& Spaeth, S. (2008). Code reuse in open source software. Management Science, 54(1), 180-193.

Han, B.M. \& Anantatmula, V.S. (2007). Knowledge sharing in large IT organisations: a case study. VINE: The Journal of Information and Knowledge Management Systems, 37(4), 421-439.

Harvey, O. \& Gray, C. (September, 2003). 2002/2003 Annual cluster profiles. Wellington, New Zealand: Positively Wellington Business.

Helmsing, A. (1999, November). Flexible specialisation, clusters and industrial districts and 'second' and 'third generation' regional policies. Working paper series no. 305. The Hague, The Netherlands: Institute of Social Studies. 
Henton, D., Melville, J. \& Walesh, K. (1997). Grassroots Leaders for a New Economy. San Francisco, USA: Jossey-Bass Publishers

Hirschheim, R.A. (1992). Information systems epistemology: An historical perspective. In Galliers, R. (Ed.), Information systems research:Iissues, methods and practical guidelines (pp.28-60). Oxford: Blackwell Scientific Publications.

Hirschheim, R.A. \& Klein, H.K. (1994). Realizing emancipatory principles in information systems development: The case for ETHICS. MIS Quarterly, 18(1), 83-109.

Hult, M. \& Lennung, S. (1980). Towards a definition of action research: A note and bibliography. Journal of Management Studies, 17, 241-250.

Hussey, J. \& Hussey, R. (1997). Business research: A practical guide for undergraduate and postgraduate students. Great Britain: Macmillan Press Ltd.

Iammarino, S. \& McCann, P. (2006). The structure and evolution of industrial clusters: Transactions, technology and knowledge spillovers. Research Policy, 35(7), 1018-1036.

Ihlström, C. \& Henfridsson, O. (2005) Online newspapers in Scandinavia: A longitudinal study of genre change and interdependency. Information Technology \& People, 18(2), 172-192.

Im, H., Yates, J. \& Orlikowski, W. (2005). Temporal coordination through communication: Using genres in a virtual start-up organization. Information Technology \& People, 18(2), 89-119.

Industry New Zealand. (2001). Annual report, 2000-2001. Wellington, New Zealand: Author. Available at: http://www.industrynz.govt.nz/aboutus/_documents/Annual-Report-2001.pdf [date accessed: April 28, 2003]

Industry New Zealand. (2002). Statement of intent 2002/03. Wellington, New Zealand: Author. Available at: http://www.industrynz.govt.nz/aboutus/_documents/statement-of-intent.pdf [date accessed: April 28, 2003].

Information Technology Advisory Group (ITAG). (August, 1999). The knowledge economy: A submission to the New Zealand Government by the Minister for Information Technology's IT Advisory Group. Wellington, New Zealand: Ernst \& Young. Available at www.med.govt.nz/pbt/infotech/knowledge_economy [Date accessed: December 10, 2002]. 
Janesick, V.J. (1988). The dance of qualitative research design. In Denzin, N.K. \& Lincoln, Y.S. (Eds.), Strategies of qualitative inquiry (pp.35-55). Thousand Oaks, CA, USA: SAGE Publications, Inc.

Jasimuddin, S. M. (2007). Exploring knowledge transfer mechanisms: The case of a UK-based group within a high-tech global corporation. International Journal of Information Management, 27(4), 294-300.

Jasimuddin, S. M., Klein J. H., Connell, C. (2005). The paradox of using tacit and explicit knowledge. Management Decision, 43(1), 102-112.

Jonscher, C. (1994). An economic study of the information technology revolution. In Allen, T. and Scott Morton, M. (Eds.), Information technology and the corporation of the 1990s (pp. 5-42). New York: Oxford University Press.

Jugdev, K. \& Müller, R. (2005). A retrospective look a tour evolving understanding of project success. Project Management Journal, 36(4), 19-31.

Jönsson, S. (1991). Action research. In Nissen, H., Klein, H.K. \& Hirschheim, R. (Eds.), Information systems research: contemporary approaches \& emergent traditions (pp.371-396). Amsterdam, The Netherlands: Elsevier Science Publishers.

Juniper, J. \& Metcalfe, M. (2000). Knowledge sharing in the industrial milieu. Working paper 2000-11. Centre of Business Analysis and Research (CoBAR). http://business.unisa.edu.au/cobar/workingpapers/cobar/200-11.pdf [date accessed: October 30, 2003].

Karev, A., Lenny Koh, S.C. \& Szamosi, L.T. (2007). The cluster approach and SME competitiveness: A review. Journal of Manufacturing Technology Management, 18(7), 818-835.

Kappelman, L., McKeeman, R. \& Zhang, L. (2006). Early warning signs of IT project failure: The dominant dozen. Information Systems Management, 23(4): 31-36.

Keane B.T. \& Mason, R. M. (2006). On the nature of knowledge: rethinking popular assumptions. In Proceedings of the 39th Hawaii International Conference on System Sciences 4-7 January 2006 (pp. 162b-162b). Los Alamitos: IEEE Press.

Klein, B.D., Goodhue, D.L. \& Davis, G.B. (1997). Can humans detect errors in data? Impact of base rates, incentives, and goals. MIS Quarterly 21(2), 169-194. 
Klein, H., Nissen, H. \& Hirschheim, R. (1991). A pluralist perspective of the information systems research arena. In Nissen, H., Klein, H.K. \& Hirschheim, R. (Eds.), Information systems research: Contemporary approaches \& emergent traditions (pp.120). Amsterdam, The Netherlands: Elsevier Science Publishers.

Kliem, R.L. (1992). Back to basics: Developing a good requirements document. Journal of Systems Management, 43(10), 16-19.

Kluge, J., Stein, W. \& Licht, T. (2001). Knowledge unplugged. Basingstoke, Great Britain: Palgrave.

Kock, N. (1997). Myths in organisational action research: Reflections on a study of computer-supported process redesign groups. Organizations \& Society, 4(9), 65-91. Available at: http://www.cis.temple.edu/ kock/public/ro\&s/ro\&s1.html [Date accessed: May 2, 2004].

Kock, N., Avison, D., Baskerville, R. Myers, M. \& Wood-Harper, T. (1999). IS action research: can we serve two masters? In Proceedings of the 20th International Conference on Information Systems, (pp. 269-289). New Yourk: The Association for Computing Machinery.

Kock, N., Baker, M., McQueen, R., \& Rouse, A. (1996). Topic negotiation and failure in IS action research in organisations. New Zealand Journal of Computing, 6(1), 26-36.

Kock, N. \& DeLuca, D. (2007). Improving business processes electronically: an action research study in New Zealand and the US. Journal of Global Information Technology Management, 10(3), 6-27.

Kock, N. (Ed.). (2007). Preface. In Kock, N. (Ed.), Information systems action research (pp. xix-xxii). New York: Springer Science.

Kock, N. \& Lau, F. (2001). Information systems action research: Serving two demanding masters. Information Technology \& People, 14(1), 6-11.

Kumar, K. \& van Dissel, H. (1996). Sustainable collaboration: Managing conflict and cooperation in interorganizational systems. MIS Quarterly, 20(3), 279-300.

Lagendijk, A. \& Charles, D. (1999). Clustering as a new growth strategy for regional economies? A discussion of new forms of regional industrial policy in the United Kingdom. In OECD proceedings boosting innovation:The cluster approach (pp. 127-153). Paris: OECD. 
Lau, F. (1997). A review on the use of action research in information systems studies. In Lee, A., Liebenau, J. \& DeGross, J. (Eds.), Information systems and qualitative research (pp. 31-68). London: Chapman \& Hall.

Lau, F. (1999). Towards a framework for action research in information systems studies. Information Technology \& People, 12(2), 148-176.

Lawrence, E., Corbitt, B., Fisher, J., Lawrence, J. \& Tidwell, A. (2000). Internet Commerce. $\left(2^{\text {nd }} E d\right)$. Brisbane, Australia: John Wiley \& Sons Australia, Ltd.

Lee, A.S. \& Liebenau, J. (1997). Information systems and qualitative research. In Lee, A.S, Liebenau, J. \& DeGross, J.I. (Eds.), Information systems and qualitative research (pp.1-8). London: Chapman \& Hall.

Lee, L. \& Al-Hawamdeh, S. (2002). Factors impacting knowledge sharing. Journal of Information \& Knowledged Management, 1(1), 49-56.

Leedy, P. (1997). Practical Research: Planning and design (6 ${ }^{\text {th }}$ ed.). New Jersey, USA: Prentice-Hall Inc.

Lesca, N. \& Caron-Fasan, M (2008). Strategic scanning project failure and abandonment factors: lessons learnt. European Journal of Information Systems 17(4), 371-386.

Levin, M. \& Greenwood, D. (2001). Pragmatic action research and the struggle to transform universities into learning communities. In Reason, P. \& Bradbury, H. (Eds.), Handbook of action research: participative inquiry and practice (pp. 103-113).

London: SAGE Publications.

Li, F. \& Williams, H. (2001). Interorganizational systems to support strategic collaboration between firms. In Barnes, S \& Hunt, B. (Eds.), E-commerce \& V-business (pp. 153-170). Oxford: Butterworth-Heinemann.

Lincoln, Y. S. \& Guba, E. G. (1985). Naturalistic Inquiry. California, USA: Sage Publications, Inc.

Lindsay, V. J. (2005). The developmnet of international industry clusters: A complexity theory approach. Journal of International Entrepreneurship 3(1), 71-97.

Liu, T. (2007). Knowledge transfer: Past research and future directions. The Business Review, Cambridge 7(1), 273-281. 
Loebbecke, C., Van Fenema, P. C. \& Powell, P. (1999). Co-opetition and knowledge transfer. The DATA BASE for Advances in Information Systems, 30(2), 1425 .

Lorenzoni, G. \& Ferriani, S. (2008). Searching for new units of analysis: Firms, dyads and networks. European Management Review, 5(2), 125-133.

Magaziner, I. \& Patinkin, M. (1989). Fast heat: How Korea won the microwave war. Harvard Business Review, January-February(1), 83-92.

Majchrzak, A., Cooper, L. \& Neece, O. (2004). Knowledge reuse for innovation. Management Science, 50(2), 174-188.

Majchrzak, A., Rice, R.E., King, N., Malhotra, A. \& Ba, S. (2000), Technology adaptation: The case of a computer-supported inter-organizational virtual team. MIS Quarterly, 24(4), 569 - 600

Malmberg, A. \& Power, D. (2005). (How) do (firms in) clusters create knowledge? Industry and Innovation, 12(4), 409-431.

Mansourian, Y. (2006). Adoption of grounded theory in LIS research. New Library World, 107(1228/1229), 386-402.

Manzari, L. \& Trinidad-Christensen. (2006). User-centered design of a web site for library and information science students: Heuristic evaluation and usability testing. Information Technology and Libraries, 25(3), 163-169.

Markus, L. (1997). The qualitative difference in information systems research and practice. In Lee, A.S, Liebenau, J. \& DeGross, J.I. (Eds.), Information systems and qualitative research (pp.1-8). London: Chapman \& Hall.

Markus, L. (2001). Toward a theory of knowledge reuse: Types of knowledge reuse situations and factors in reuse success. Journal of Management Information Systems, 18(1), 57-93.

Markusen, A. (1996). Sticky places in slippery space: A typology of industrial districts. Economic Geography, 72(3), 293-313.

Maskell, P. (2001). Towards a knowledge-based theory of the geographical cluster. Industrial and Corporate Change, 10(4), 921-943.

Maskell, P. \& Malmberg, A. (2007). Myopia, knowledge development and cluster evolution. Journal of Economic Geography, 7(5), 603-618. 
McDonald, F. \& Vertova G. (2001). Geographical concentration and competitiveness in the European Union. European Business Review, 13(3), 157-165.

McKay, J. \& Marshall, P. (2001). The dual imperatives of action research. Information Technology \& People, 14(1), 46-59.

McNiff, J. (1988). Action research: Principles and practice. London: Macmillan Education Ltd.

Mei, S. \& Nie, M. (2007). Relationship between knowledge sharing, knowledge characteristics, absorptive capacity and innovation: An empirical study of Wuhan optoelectronic cluster. The Business Review, Cambridge, 7(2), 154-161.

Miles, M.B. \& Huberman, A.M. (1999). Qualitative data analysis (2 ${ }^{\text {nd }}$ ed.). Thousand Oaks, CA, USA: SAGE Publications, Inc.

Minbaeva, D.B. (2007). Knowledge transfer in multinational corporations. Management International Review, 47(4), 567-593.

Ministry of Economic Development (MED). (2002). Statement of intent 20022005. (Report No. G.46 SOI-2002). Wellington, New Zealand: Author. Available at: http://www.med.govt.nz/about/soi/2002/soi2002.pdf [Date accessed: April 25, 2003]

Ministry of Economic Development (MED). (June, 2003). Regional partnerships programme review - background report. Wellington, New Zealand: Author. Available at: http://www.med.govt.nz/templates/MultipageDocumentTOC__5274.aspx [Date accessed: April 28, 2008]

Ministry of Economic Development (MED). (September, 2005). Cluster development programme review. Wellington, New Zealand: Author. Available at: http://www.med.govt.nz/upload/35254/cdp.pdf [Date accessed: April 28, 2008]

Ministry of Economic Development (MED). (May, 2007). Statement of intent 2007-2010. (Report No. G.46 SOI-2007) Wellington, New Zealand: Author. Available at: http://www.med.govt.nz/templates/MultipageDocumentTOC 26924.aspx [Date accessed: June 7, 2007]

Ministry of Economic Development (MED) \& The Treasury. (2005). Economic Development Indicators. Wellington, New Zealand: Authors. Available at: http://gif.med.govt.nz/aboutgif/indicators-2005/report/report.pdf [Date accessed: May $21,2007]$ 
Molina M. \& Yoong. P. (2003). Knowledge sharing in a co-opetitive environment: The case of business clusters. Journal of Information \& Knowledge Management, 2(4), 321-341.

Morse, J.M. (1998). Designing funded qualitative research. In Denzin, N.K. \& Lincoln, Y.S. (Eds.), Strategies of qualitative inquiry (pp.56-85). Thousand Oaks, CA, USA: SAGE Publications, Inc.

Morton, A. (1999). Ethics in action research. Systemic Practice and Action Research, 12(2), 219-222.

Mumford, E. (2001). Advice for an action researcher. Information, Technology \& People, 14(1), 12-27.

Muscio, A. (2006). Patterns of innovation in industrial districts: An empirical analysis. Industry and Innovation, 13(3), 291-312.

Nah, F.F. \& Lau, J.L. (2001). Critical factors for successful implementation of enterprise systems. Business Process Management Journal, 7(3), 285-296.

Nalebuff, B. \& Brandenburger, A. (1996). Co-opetition. London: HarperCollinsBusiness.

Narayanaswamy, R. \& Grover, V. (2007). A critical assessment of information systems action research. In Kock, N. (Ed.), Information systems action research (pp. 327-353). New York: Springer Science.

Newell, S. (2004). Enhancing cross-project learning. Engineering Management Journal, 16(1), 12-20.

Newell, S., Bresnen, M., Edelman, L., Scarbrough, H. \& Swan, J. (2006). Sharing knowledge across projects. Management Learning, 37(2), 167-185.

Ngwenyama, O.K. \& Lee, A.S. (1997). Communication richness in electronic mail: Critical social theory and the contextuality of meaning. MIS Quarterly, 21(2), 145-167.

Nonaka, I. \& Konno, N. (1998). The concept of "Ba": Building a foundation for knowledge creation. California Management Review, 40(3), 40-54.

Nonaka, I., Toyama, R. \& Konno, N. (2000). SECI, Ba and leadership: A unified model of dynamic knowledge creation. Long Range Planning, 33(1), 5-34. 
Ojiako, U., Johansen, E. \& Greenwood, D. (2008). A qualitative re-construction of project measurement criteria. Industrial Management \& Data Systems, 108(3), 405-417.

Olesen, K. \& Myers, M. D. (1999). Trying to improve communication and collaboration with information technology. Information Technology \& People, 12(4), 317-332.

Organisation for Economic Co-operation and Development (OECD). (1998). Technology, productivity and job creation. Paris: OECD.

Organisation for Economic Co-operation and Development (OECD). (1999). Managing national innovation systems. Paris: OECD.

Orlikowski, W. J. \& Baroudi, J.J. (1991). Studying information technology in organisations: Research approaches and assumptions. Information Systems Reasearch, 2(1), 1-28.

Orlikowski, W.J. \& Yates, J. (1994). Genre repertoire: The structuring of communicative practices in organizations. Administrative Science Quarterly, 39(4), 541-574.

Østerlund, C. (2006). Combining genres: How practice matters. In Proceedings of the 39th Hawaii International Conference on System Sciences 4-7 January 2006 (pp. 10). Los Alamitos: IEEE Press.

Owen, J. M. (2006). Program evaluation: Forms and approaches. ( $3^{\text {rd }}$ ed.). New York: Guilford Press.

Owen, J., Burstein, F. \& Mitchell, S. (2004). Knowledge reuse and transfer in a project management environment. Journal of Information Technology Cases and Applications, 6(4), 21-35.

Park, S-C. \& Lee, S-K. (2004). The regional innovation system in Sweden: A study of regional clusters for the development of high technology. AI \& Society, 18(3), 276-292.

Parto, S. (2008). Innovation and economic activity: An institutional analysis of the role of clusters in industrializing economies. Journal of Economic Issues, 42(4), 10051030 .

Pavlovich, K. \& Akoorie, M. (2005). Cluster analysis: Mapping the Nelson seafood industry. University of Auckland Business Review, 7(2), 55-63. 
Pearlson, K. E., \& Saunders, C. S. (2004). Managing and using information systems: A strategic approach. ( $2^{\text {nd }}$ ed.). New York: John Wiley \& Sons, Inc.

Perry, M. (2007). Seeing trees and forests: A comparative evaluation of business clusters and national industry associations in the New Zealand forest sector. Innovation: Management, Policy \& Practice, 9(1), 46-61.

Pinch, S., Henry, N., Jenkins, M. \& Tallman. (2003). From 'industrial districts' to 'knowledge clusters': A model of knowledge dissemination and competitive advantage in industrial agglomeration. Journal of Economic Geography, 3(4), 373-388.

Pollach, I. (2006) Electronic word of mouth: A genre analysis of product review on consumer opinion web sites. In Proceedings of the 39th Hawaii International Conference on System Sciences 4-7 January 2006 (pp. 10). Los Alamitos: IEEE Press.

Porter, M. E. (1990). The competitive advantage of nations. New York: The Free Press.

Porter, M. E. (1998a). On competition. Boston: Harvard Business School Press.

Porter, M. E. (1998b). Clusters and the new economics of competition. Harvard Business Review 76(November-December), 77-90.

Pöyhönen, A. \& Smedlund, A. (2004). Assessing intellectual capital creation in regional clusters. Journal of Intellectual Capital, 5(3), 351-365.

Prasse, M. (1991). Achieving better systems development through usability testing. Journal of Systems Management, 42(9), 10-12.

Probst, G., Raub, S. \& Romhardt, K. (2000). Managing knowledge: Building blocks for success. Chichester, England: John Wiley \& Sons, Ltd.

Quandt, C. (1999). Virtual technopoles: Exploring the potential of Internet and Web technologies to create innovative environments in Latin America and the Caribbean. In IDRC/CRDI: Pan American Consultation. 11-12 December Montevideo, Uruguay.

Reason, P. \& Bradbury, H. (2001). Introduction: inquiry and participation in search of a world worthy of human aspirations. In Reason, P. \& Bradbury, H. (Eds.), Handbook of action research: participative inquiry and practice (pp. 1-14). London: SAGE Publications. 
Rhem, G. (2002). Towards automatic web genre identification. In Proceedings of the 35th Hawaii International Conference on System Sciences, 7-10 January 2002 (pp. 10). Los Alamitos: IEEE Press.

Roberts, B.H. \& Enright, M.J. (2004). Industry clusters in Australia: recent trends and prospects. European Planning Studies, 12(1), 99-121.

Roelandt, J \& den Hertog, P. (1999). Cluster analysis and cluster-base policy making in OECD countries: An introduction to the theme. In OECD proceedings boosting innovation: the cluster approach (pp. 9-23). Paris: OECD.

Rolland, N. \& Chauvel, D. (2000). Knowledge transfer in strategic alliances. In Despress \& Chauvel (Eds.), Knowledge horizons: the present and the promise of knowledge management. Woburn, MA, USA: Butterworth-Heinemann.

Romanelli, E. \& Khessina, O.M. (2005). Regional industrial identity: Cluster configurations and economic development. Organization Science, 16(4), 344-358.

Romer, P. (1986). Increasing returns and long-run growth. Journal of Political Economy, 94(5), 1002-1037.

Romer, P. (1990). Endogenous technological change. Journal of Political Economy, 98(Suppl. 5), S71-S102.

Rosenfeld, S. (1997). Bringing business clusters into the mainstream of economic development. European Planning Studies, 5(1), 3-24.

Rosenthal, S.S. \& Strange, W.C. (2003). Geography, industrial organization, and agglomeration. The Review of Economics and Statistics, 85(2), 377-393.

Rupino da Cunha, P. \& Dias de Figueiredo, A. (2002). Action-research and critical rationalism: a virtuous marriage. In Proceedings of the 10th European Conference on Information Systems, 6-8 June 2002 (pp. 19-27). Gdańsk, Poland: ECIS.

Santos, R. (2006). Interaction between innovation systems, clustering and sustainability: Regional implications. Internaltional Journal Foresight and Innovation Policy, 2(2). 184-198.

Scott, J. (2000). Facilitating interorganisational learning with information technology. Journal of Management Information Systems, 17(2), 81-113.

Schiele, H. (2008). Location, location: The geography of industry clusters. Journal of Business Strategy, 29(3), 29-36. 
Shepherd, M. \& Watters, C. (1998). The Evolution of Cybergenre. Proceedings 31 st Hawaii International Conference on Systems Science 6-9 January 1998 (pp. 97109.

Simmie, J. (2003). Innovation and urban regions as nations and international nodes for the transfer and sharing of knowledge. Regional Studies, 37(6\&7), 607-620.

Simmie, J. \& Sennett, J. (1999). Innovative clusters: global or local linkages? National Institute Economic Review, 170(1), 87-98.

Simon, S.J. (2000). The reorganization of the information systems of the US naval construction forces: an action research project. European Journal of Information Systems, 9(3), 148-162.

Singh, S.P. (2007). What are we managing - knowledge or information? VINE: the Journal of Information and Knowledge Management Systems, 37(2), 169-179.

Sipior, J. (2000). Expert system stalemate: A case of project champion departure. Information Resources management Journal, 13(4), 16-24.

Smedlung, A. (2006). The roles of intermediaries in a regional knowledge system. Journal of Intellectual Capital, 7(2), 204-220.

Smeds, R., Olivari, P. \& Corso, M. (2001). Continous learning in global product development: A cross-cultural comparison. International Journal of Technology Management, 22(4), 373-392

Snow, C., Miles, R. \& Coleman, H. (1992). Managing $21^{\text {st }}$ century network organizations. Organizational Dynamics, 2(3), 5-16.

Snyder, C, \& Wilson, L. (2000). Implementing knowledge management: Issues for managers. In Challenges of Information Technology Management in the $21^{\text {st }}$ Century International Conference 21-24May 2000 (pp.527-530). Anchorage, Alaska, USA: Information Resource Management Association.

Sölvell, Ö., Lindqvist, G. \& Ketels, C. (2003). The cluster initiative greenbook. Stockholm, Sweden: Bromma tryck AB.

Sommer, R. \& Sommer, B. (2002). A practical guide to behavioral research: Tools and techniques. New York: Oxford University Press.

Steinfield, C. (2002). Conceptualizing the role of collaborative e-commerce in geographically defined business clusters. For presentation at the research workshop "The 
Ambivalent Relationship Between Social Capital and IT". Amsterdam, The Netherlands. Available at: http://www.msu.edu/ steinfie/B2Bsocialcapital.pdf [Date accessed: March 17, 2004]

Strauss, A.L. \& Corbin, J. (1994). Grounded theory methodology: An overview. In Denzin, N.K. \& Lincoln, Y.S. (Eds.), Handbook of Qualitative Research (pp. 273285). Thousand Oaks, California, USA: SAGE Publications Inc.

Su, Y. \& Hung, L. (2009). Spontaneous vs. Policy-driven: The origin and evolution of the biotechnology cluster. Technological Forecasting \& Social Change, 76(5): 608-619.

Susman, G.I. \& Evered, R.D. (1978). An assessment of the scientific merits of action research. Administrative Science Quarterly, 23(4): 582-603.

Sutterfield, J., Friday-Stroud, S.S. \& Shivers-Blackwell, S.L. (2006). A case study of project and stakeholder management failures: Lessons learned. Project Management Journal, 37(5): 26-35.

Sveiby, K. (1997). The new organizational wealth: Managing \& measuring knowledge-based assets. San Francisco, USA: Berret-Koehler Publishers, Inc.

Tallman, S., Jenkins, M., Henry, N. \& Pinch, S. (2004). Knowledge, clusters and competitive advantage. Academy of Management Review, 29(2), 258-271.

Taylor, H. (2007). Tacit knowledge: Conceptualizations and operationalizations. International Journal of Knowledge Management, 3(3), 60-73.

TFPL. (1999). Skills for knowledge management - building a knowledge economy. A report by TFPL Ltd. London: TFPL.

The Design Taskforce (May, 2003). Success by design: A report and strategic plan. Wellington, New Zealand: Ministry of Industry and Regional Development.

The Office of the Prime Minister. (2002). Growing an innovative New Zealand. Wellington, New Zealand: The Office of the Prime Minister. Available: http://www.executive.govt.nz/minister/clark/innovative/index.html [date accessed: Dec $1,2002]$.

Tricker, R.I. (1992). The management of organisational knowledge. In Galliers, R. (Ed.), Information systems research: issues, methods and practical guidelines (pp.1427). Oxford: Blackwell Scientific Publications. 
Turban, E., \& King, D. (2003). Introduction to e-commerce. Upper Saddle River, NJ, USA: Prentice Hall.

Turban, E, McLean E., \& Wetherbe, J. (2001). Information Technology for Management. New York: John Wiley.

United Nations Conference on Trade and Development (UNCTAD). (1997). An overview of activities in the area of inter-firm cooperation. (Report No. GE.97-51735). Geneva, Switzerland: UNCTAD. Available at:

http://www.unctad.org/en/docs//poiteedsd2.en.pdf [Date accessed: April 25, 2003]

Van de Ven, A.. (2005). Running in packs to develop knowledge-intensive technologies. MIS Quarterly, 29(2), 365-378.

Venkatraman, N. (1991). IT-Induced business reconfiguration. In Scott Morton, M. (Ed.), The corporation of the 1990s: Iinformation technology and organizational transformation (pp.122-158). New York: Oxford University Press.

Vera-Muñoz, S.C., Ho, J.L. \& Chow, C.W. (2006). Enhancing knowledge sharing in public accounting firms. Accounting Horizons, 20(2), 133-155.

Wang, S. (2001) Toward a general model for web-based information systems. International Journal of Information Management, 21(5), 385-396.

Waits, M. J. (2000). The added value of the industry cluster approach to economic analysis, strategy development, and service delivery. Economic Development Quarterly, 14(1), 35-50.

Watson, J. \& Fenner, J. (2000). Understanding portals. The Information Management Journal, 34(15), 18-22.

Wellington, R. (2002). Asynchronous communication technology: An organisational perspective on efficacy and use. Unpublished doctoral dissertation, Waikato University, Hamilton, New Zealand.

Wellington Regional Economic Development Trust (WREDA). (2002a). 2002/03 Business plan. Wellington, New Zealand: Author. Available: http://www.smartwellington.co.nz/PDF/REDA\%20Business\%20Plan.pdf [Date accessed: April 28, 2003].

Wellington Regional Economic Development Trust (WREDA). (September, 2002b). New clusters win contracts. Smart Wellington, Issue 5, 6. 
West, D. \& Stansfield, M.H. (2001). Structuring action and reflection in information systems action research studies using Checkland's FMA model. Systemic Practice and Action Research, 14(3), 251-281.

Whitmore E. \& McKee C. (2001). Six street youth who could... In Reason, P. \& Bradbury, H. (Eds.), Handbook of action research: Participative inquiry and practice (pp. 396-402). London: Sage Publications.

Wielemaker, M. W., Elfring, T., Volberda, H. W. (2001). How well-established firms prepare for the new economy. International Studies of Management \& Organisation, 31(1), 7-29.

Wong, F., Loke, A., Wong, M., Tse, H., Kan, E. \& Kember, D. (1997). An action research study into the development of nurses as reflective practitioners. Journal of Nursing Education, 36(10), 476-481.

Yang, H. \& Tang, J. (2003). A three-stage model of requirements elicitation for web-based information systems. Industrial Management + Data Systems, 103(6), 398409.

Yates, J. \& Benjamin, R. I. (1991). The past and present as a window on the future. In Scott Morton, M. (Ed.), The corporation of the 1990s: information technology and organizational transformation (pp.61-92). New York: Oxford University Press.

Yates, J. \& Orlikowski, W. (1992). Genres of organizational communication: A structurational approach to studying communication and media. The Academy of Management Review, 17(2), 299-326.

Yates, J. \& Orlikowski, W. (2002). Genre systems: Structuring interaction through communicative norms. The Journal of Business Communication, 39(1), 13-35.

Yates, J., Orlikowski, W. \& Okamura, K. (1999). Explicit and implicit structuring of genres in electronic communication: Reinforcement and change of social interaction. Organization Science, 10(1), 83-103.

Yin, R. (1994) Case study research: Design and methods ( $2^{\text {nd }}$ ed.). Thousand Oaks, California, USA: SAGE Publications Inc.

Yoong, P. (1996). A grounded theory of reflective facilitation: Making the transition from traditional to GSS facilitation. Unpublished doctoral dissertation. Wellington, New Zealand: Victoria University of Wellington. 
Yoong, P. \& Gallupe B. (2001) The emergence of a theoretical framework for GSS facilitation: the dualities of e-facilitation. Journal of Systems and Information Technology, 5(1), 59-80.

Zhang, M.J. (2007) An empirical assessment of the performance impacts of IS support for knowledge transfer. International Journal of Knowledge Management, 3(1), 66-85.

Zhang, J. \& Faerman, S. R. (2007). Distributed leadership in the development of a knowledge sharing system. European Journal of Information Systems, 16(4), 479-493.

Zuber-Skerritt, O. \& Perry, C. (2002). Action research within organisations and university thesis writing. The Learning Organisation, 9(3/4), 171-179. 


\section{Acronyms}

\begin{tabular}{|l|l|}
\hline ADB & Asian Development Bank \\
\hline CDP & Cluster Development Programme \\
\hline EENZ & Earthquake Engineering New Zealand \\
\hline EOI & Expression of Interest \\
\hline ITAG & Information Technology Advisory Group \\
\hline MED & Ministry of Economic Development \\
\hline NHNZ & Natural Hazards New Zealand \\
\hline NZAID & New Zealand Aid \\
\hline NZTE & New Zealand Trade and Enterprise \\
\hline OCED & Organisation for Economic Co-operation and Development \\
\hline PWB & Positively Wellington Business \\
\hline
\end{tabular}


Appendixes 


\section{Appendix A: Interview tools}

\section{Questionnaire for the Diagnosis stage of the study}

Project Title: Facilitating knowledge sharing in business clusters: the role of information and communication technologies.

1. Review the steps or activities outlined in the attached diagrams, which aim to illustrate a typical project bidding process by the cluster.

2. For each of the steps or activities identified for the project bidding process, what are the information and communication channels or mechanisms that are currently employed to exchange the pertinent knowledge or information?

3. What kind of knowledge and/or information is usually shared during the project bidding process?

4. How does this knowledge/information flow between participants?

5. What do you think about the current process and the information and communication channels? Do you think knowledge and information flow efficiently and effectively to where it is needed?

6. If question 5 is negative, which parts of the bidding process do you think should be improved?

7. What information and communication technologies would you like to see being implemented in order to improve the project bidding/marketing process?

8. What could be some potential issues and challenges that need to be considered if a decision was made to improve the system for project bidding?

9. Do you think there is support by the rest of the cluster members to work towards improving the current project bidding process? Why or why not?

\section{Questionnaire for the Website Planning stage of the study}

Project Title: Facilitating knowledge sharing in business clusters: the role of information and communication technologies.

The second stage of the study involves developing a new website for Natural Hazards New Zealand (NHNZ). The website will enable NHNZ engage in marketing and collaboration activities, as well as providing a repository for cluster documents. The following questions were designed to collect information and opinion about the "old" and the "new" NHNZ websites.

1. When was the "old" NHNZ website created?

2. How would you describe the "old" website? What was/were its purpose/s?

3. Why the 1-page CVs (or profiles) have been important for the cluster? How these profiles have been used? 
4. According to the NHNZ Minutes for the meeting held on 21 August 2003, the cluster initial strategy was to promote individual specialists. Would it be valid to say that this strategy was reflected in the "old" website, where visitors could view 1-page profiles of experts?

5. Why did the cluster decide to review their website?

6. The "new" website is more complex than the "old" one, in terms of content and features. What is your opinion?

7. Do you think cluster members will find this website useful for their project bidding process? How?

8. Do you think the "new" website and the database could be used to share and reuse knowledge during project bidding?

9. What are the issues and challenges that the website may encounter to be adopted by users as a tool to assist them during project bidding?

\section{Questionnaire for the Website Development stage of the study}

Project Title: Facilitating knowledge sharing in business clusters: the role of information and communication technologies.

This stage of the study is developing a new website for Natural Hazards New Zealand (NHNZ). The website will enable NHNZ engage in marketing and collaboration activities, as well as providing a repository for cluster documents. A prototype for this website has been developed and recently tested by a small group of cluster members. The following questions were designed to collect their views and suggestions on how to improve the website.

1. How did you find the process of entering CVs in the website?

2. How has been the response of cluster members to your request for their CVs and asking them to review the information?

3. Do you see a value using the website and the database for the cluster? In particular for project bidding?

4. Do you think cluster members will find this website useful for their project bidding process?

5. What are the issues and challenges that the website may encounter to be adopted by users?

\section{Questionnaire for the Website Evaluation stage of the study Testing the prototype}

Project Title: Facilitating knowledge sharing in business clusters: the role of information and communication technologies. 
This stage of the study is developing a new website for Natural Hazards New Zealand (NHNZ). The website will enable NHNZ engage in marketing and collaboration activities, as well as providing a repository for cluster documents. A prototype for this website has been developed and recently tested by a small group of cluster members. The following questions were designed to collect the tester's views and suggestions on how to improve the prototype.

1. What you did as preparation for the test and then when testing the NHNZ website?

2. How did you find the process of entering CVs in the website?

3. Were the instructions in the forms easy to understand and follow? Did the forms follow a logical flow?

4. Were there any programming errors in the database? Please describe them.

5. Do you have any suggestions on how to improve the forms and the database?

6. Do you see a value using the website and the database for the cluster?

\section{Questionnaire for the Website Evaluation stage of the study}

Project Title: Facilitating knowledge sharing in business clusters: the role of information and communication technologies.

This stage of the study is evaluating the new website for Natural Hazards New Zealand (NHNZ). The website enables NHNZ members to engage in marketing and collaboration activities, as well as providing a repository for cluster documents. The following questions were designed to collect your views and suggestions on how you use the website and how to improve it.

1. When was the last time you visited the NHNZ website?

2. What did you do?

3. Describe a specific experience of using the NHNZ website.

4. Have you used the resources available in the website?

5. What could be done to improve this website? 


\title{
Appendix B: Project evaluation tool
}

\author{
Natural Hazards New Zealand (NHNZ) \\ Website Progress Update (September, 28, 2004)
}

The following table shows the Task Group initial requirements, what GNS agreed to provide as part of their contract with NHNZ, and finally what has been achieved up to this date. The black smiley-face means that the item has been produced; the white smiley-face indicates that work is been done on the item; and finally no smiley-face means that this item has not been done yet. The table is based on a visit to the prototype Website on Tuesday, September 28, 2004 and therefore some items may already have been produced without been setup for online access. This table was prepared to help the Task Group review the progress of the Website development. It is expected that this table will have modifications and corrections during the Task Group meeting in October $1^{\text {st }}, 2004$.

\begin{tabular}{|c|c|c|c|}
\hline TABLE 1. Summary of the Website & $\begin{array}{c}\text { Task } \\
\text { Group }\end{array}$ & Contract & $\begin{array}{c}\text { Item } \\
\text { Produced }\end{array}$ \\
\hline \multicolumn{4}{|l|}{ The Website structure: } \\
\hline - A new design for the NHNZ Website & $\mathrm{X}$ & $\mathrm{X}$ & $\odot$ \\
\hline - Consistent look and feel & $\mathrm{X}$ & $\mathrm{X}$ & (-) \\
\hline $\begin{array}{l}\text { - A simple structure for efficient maintenance of the Web pages and lessen } \\
\text { reliance on original creator }\end{array}$ & $\mathrm{X}$ & & (-) \\
\hline $\begin{array}{l}\text { - Design a setup that enables cluster members to create new track records and } \\
\text { CV's online }\end{array}$ & $\mathrm{X}$ & $\mathrm{X}$ & $\odot$ \\
\hline \multicolumn{4}{|l|}{ The Website navigation: } \\
\hline - Good and consistent navigation across the site & $\mathrm{X}$ & $\mathrm{X}$ & (:) \\
\hline $\begin{array}{l}\text { - Dynamically generated links to enter into the 'database' by product, service or } \\
\text { hazard }\end{array}$ & & $\mathrm{X}$ & $\odot$ \\
\hline \multicolumn{4}{|l|}{ The Website content (i.e. pages) } \\
\hline - Home Page & $\mathrm{X}$ & $\mathrm{X}$ & (๑) \\
\hline - Who we are & $\mathrm{X}$ & $\mathrm{X}$ & $\odot$ \\
\hline - NZNH Contact details & $\mathrm{X}$ & & () \\
\hline - List of company members & $\mathrm{X}$ & $\mathrm{X}$ & $\odot$ \\
\hline - Description of each company member with link to member's own URL & $\mathrm{X}$ & $\mathrm{X}$ & (-) \\
\hline - Dynamically generated page for each person in the cluster (public) & $\mathrm{X}$ & $\mathrm{X}$ & \\
\hline $\begin{array}{l}\text { - Dynamically generated CVs for each person in the cluster available for } \\
\text { download in } 1 \text { export format (members only) }\end{array}$ & $\mathrm{X}$ & $\mathrm{X}$ & \\
\hline - NZNH by service & $\mathrm{X}$ & $\mathrm{X}$ & () \\
\hline - NHNZ by hazard & $\mathrm{X}$ & $\mathrm{X}$ & (๑) \\
\hline - NHNZ by country/region & $\mathrm{X}$ & $\mathrm{X}$ & \\
\hline - NHNZ products & $\mathrm{X}$ & $\mathrm{X}$ & (-) \\
\hline - Overseas visitors (basic information in different languages) & $\mathrm{X}$ & & \\
\hline - News items ('blog' or similar functionality) & $\mathrm{X}$ & $\mathrm{X}$ & \\
\hline - Interesting links & $\mathrm{X}$ & & \\
\hline - Repository for marketing material (public access) & $\mathrm{X}$ & & \\
\hline \multicolumn{4}{|l|}{ The Website administration: } \\
\hline - An administration area (add/remove companies, users, projects) & $\mathrm{X}$ & $\mathrm{X}$ & (๑) \\
\hline - User authentication (login pages, etc.) & $\mathrm{X}$ & $\mathrm{X}$ & () \\
\hline - A company details page where primary contact can alter company data & $\mathrm{X}$ & $\mathrm{X}$ & () \\
\hline - A personal details page where individuals can update their own CVs & $\mathrm{X}$ & $\mathrm{X}$ & () \\
\hline \multicolumn{4}{|l|}{ Other Web functions: } \\
\hline - Use of forms for customers to email the cluster or its members for enquiries & $\mathrm{X}$ & & \\
\hline - A track-record systems & $\mathrm{X}$ & $\mathrm{X}$ & () \\
\hline $\begin{array}{l}\text { - A 'data-share' system for the semi-permanent storage of document which will } \\
\text { be accessible only to other cluster members }\end{array}$ & $\mathrm{X}$ & $\mathrm{X}$ & \\
\hline $\begin{array}{l}\text { - Secure repository to post files so that members - and clients at times - can } \\
\text { collect large documents (restricted access) }\end{array}$ & $\mathrm{X}$ & & \\
\hline - Site usage statistics & $\mathrm{X}$ & $\mathrm{X}$ & \\
\hline $\begin{array}{l}\text { - Place to post the Asian Development Bank's and the World Bank's regular } \\
\text { updates so that they can be highlighted by members }\end{array}$ & $\mathrm{X}$ & & \\
\hline - Latest Hazard Watch information included in the site & $\mathrm{X}$ & $\mathrm{X}$ & \\
\hline - Access to GNS's mail list software - details to be negotiated. & $\mathrm{X}$ & $\mathrm{X}$ & \\
\hline - Search capability & $\mathrm{X}$ & & \\
\hline - Reciprocal syndication between NHNZ and Positively Wellington Business & $\mathrm{X}$ & & \\
\hline \multicolumn{4}{|l|}{ Support: } \\
\hline $\begin{array}{l}\text { - Ongoing support for bugs and systems infrastructure upgrade, whilst the } \\
\text { NHNZ site is hosted at GNS (not including extensions, enhancements or } \\
\text { changes beyond the development period) }\end{array}$ & & $\mathrm{X}$ & $\odot$ \\
\hline - One year hosting of the NHNZ website on GNS's infrastructure & $\mathrm{X}$ & $\mathrm{X}$ & $\odot$ \\
\hline
\end{tabular}




\section{Appendix C: \\ Reactive project bidding process for EENZ and NHNZ Description of the activities involved during the process}

\begin{tabular}{|c|c|}
\hline Activity & Description \\
\hline $\begin{array}{l}\text { R.1. } \\
\text { Search and } \\
\text { identify new } \\
\text { opportunities } \\
\text { funded by } \\
\text { World Bank, } \\
\text { ADB, or } \\
\text { similar } \\
\text { institutions. }\end{array}$ & $\begin{array}{l}\text { World Bank and Asian Development Bank (ADB) expressions of interest are not usually a } \\
\text { surprise when the cluster members are keeping a close eye on what is going on in their markets of } \\
\text { interest. } \\
\text { - World Bank first does assessment project reports. After that they will set up the procurement } \\
\text { authority (if one is not already in place), which will be calling for expressions of interest (EOI) for } \\
\text { consultants who wish to make the procurement bids. After that the work for consultants will } \\
\text { emerge. } \\
\text { - NHNZ and EENZ pay an annual fee to New Zealand Trade \& Enterprise (NZTE) for World Bank } \\
\text { and ADB Monitoring Liaison Services. } \\
\text { - NZTE tracks World Bank and ADB assessment reports, news releases and procurement notices of } \\
\text { what they are planning to do around the world and sends messages to Positively Wellington } \\
\text { Business (PWB). PWB will then circulate these notices through a distribution list. } \\
\text { - Clusters will also use their contacts in those countries to gain some knowledge about what kind of } \\
\text { projects might soon be announced. } \\
\text { - There is a space during cluster meetings (Marketing Initiatives) where members talk about specific } \\
\text { markets, World Bank and ADB activities. }\end{array}$ \\
\hline $\begin{array}{l}\text { R.2. } \\
\text { Expression of } \\
\text { interest (EOI) } \\
\text { is announced }\end{array}$ & $\begin{array}{l}\text { World Bank, ADB, or similar institutions fund projects and they openly call for consultants to } \\
\text { register their interest and submit an EOI through their website or newsletters. } \\
\text { - Arrangement with PWB to get notices from World Bank and ADB every few days. PWB staff } \\
\text { collects announcements from all these various sources and circulate them to all cluster members. }\end{array}$ \\
\hline $\begin{array}{l}\text { R.3. } \\
\text { Cluster is } \\
\text { alerted }\end{array}$ & $\begin{array}{l}\text { - In the reactive approach, a member identifies an opportunity from the projects announced by } \\
\text { World Bank or ADB. } \\
\text { - Is an informal process; any member can make the alert call about the opportunity. The cluster } \\
\text { member notifies the facilitator, PWB, any of the cluster co-chairs, and bigger players in the } \\
\text { cluster. } \\
\text { - Some of the bigger cluster members are directly subscribed to the monitoring services from } \\
\text { Washington and have their own internal system in place to alert their staff of potential EOI } \\
\text { opportunities. However, they still find it useful getting the announcements from PWB and being } \\
\text { alerted by the cluster facilitator or a member. }\end{array}$ \\
\hline $\begin{array}{l}\text { R.4. } \\
\text { Informal } \\
\text { networking of } \\
\text { interested } \\
\text { members }\end{array}$ & $\begin{array}{l}\text { - Contact other cluster members in an informal manner to find out if there is interest in looking at } \\
\text { the new opportunity. } \\
\text { - Usually the cluster member who is prepared to lead the bid would initiate the networking. } \\
\text { - The interested parties agree to meet to discuss the viability of the project a bit further. }\end{array}$ \\
\hline $\begin{array}{l}\text { R.5. } \\
\text { First meeting } \\
\text { to discuss the } \\
\text { viability of } \\
\text { the } \\
\text { opportunity }\end{array}$ & $\begin{array}{l}\text { - One of the interested parties will call for a meeting at their location (usually it is the cluster } \\
\text { member who is prepared to lead the bid). } \\
\text { - First meeting is open for any cluster member interested on the project to come along. } \\
\text { - Discuss the project. Questions that may arise are: Does it sound like a real opportunity? Do we } \\
\text { have companies that are excited about this project? Do we have a chance of winning it? } \\
\text { - Some initial assessment of the project's risks, rewards, and whether the cluster has the capability } \\
\text { and capacity required for the project. } \\
\text { - Initial look at who could be the potential bidding team, who may be interested in co-ordinating the } \\
\text { putting together of the EOI, and even some thinking on the type of methodology to approach the } \\
\text { opportunity. }\end{array}$ \\
\hline
\end{tabular}




\begin{tabular}{|c|c|}
\hline Activity & Description \\
\hline $\begin{array}{l}\text { R.6. } \\
\text { Research } \\
\text { further details } \\
\text { of the } \\
\text { opportunity }\end{array}$ & $\begin{array}{l}\text { - EOIs are generally brief in describing the project; therefore cluster members need to research the } \\
\text { opportunity further. It would be helpful if the cluster has been following up the events of the } \\
\text { particular market. } \\
\text { - This step could be a cascade of further interactions with various bits of information being brought } \\
\text { back and shared. } \\
\text { - Scope out a bit more detail based on the action points defined in the first meeting. } \\
\text { - Try to get more information about the project (e.g. who is doing the calling for bids) by emailing } \\
\text { and phoning some contacts in the country. Contacts may be the local NZTE Commission, local } \\
\text { partners, and known government officials. } \\
\text { - Gathering information may happen very quickly for projects funded by World Bank or ADB as } \\
\text { there is usually not much time available to submit the Expression of Interest. }\end{array}$ \\
\hline $\begin{array}{l}\text { R.7. } \\
\text { Confirm } \\
\text { member who } \\
\text { will lead bid }\end{array}$ & $\begin{array}{l}\text { - Sometimes it is a straightforward step as one company offers to lead the EOI since the opportunity } \\
\text { has been identified. Other times it is not clear who will lead the group, but eventually this is sorted } \\
\text { out. } \\
\text { - It is important to put forward the strongest person and the strongest entity to increase the chances } \\
\text { of being short-listed. } \\
\text { - It is based on overseas connections and a track record. The lead company needs to have a high } \\
\text { degree of sophistication and experience in bidding in the international arena. It also needs to have } \\
\text { some kind of professional indemnity insurance. } \\
\text { - Leading the EOI is a commitment from the company to lead the bid if the cluster's proposal gets } \\
\text { short-listed. This because a huge proportion of the client's decision to short-list a candidate is } \\
\text { based on the qualities of the lead company. }\end{array}$ \\
\hline $\begin{array}{l}\text { R.8. } \\
\text { Confirm team } \\
\text { for EOI }\end{array}$ & $\begin{array}{l}\text { - At this stage, it is basically a commitment of names to go down in the EOI with a brief description } \\
\text { of their company and their individual profile. } \\
\text { - It is a self-selecting process. Each individual analyses the opportunity and reflects on whether they } \\
\text { have something to contribute to the project. It is more through attrition, or acknowledgement by } \\
\text { all parties who the best team of people is rather than going through a formal selection process. } \\
\text { - The EOI only provides a broad scope of the work so the cluster is speculating on what is the mix } \\
\text { of experts required for the particular job is. At this stage there is no real assurance that those } \\
\text { individuals listed for the EOI would actually be in the team that is going to bid for the project later } \\
\text { on if they get short-listed. The exception is the lead company and in particular the person they put } \\
\text { forward to be in charge of this EOI as already discussed in R.7. } \\
\text { - It could happen that there is a lot of interest from various members with similar skills and } \\
\text { expertise and therefore it would come down to CVs. Usually the situation is the opposite though: } \\
\text { the few who are interested in the project at the beginning realise that they need the expertise of } \\
\text { other cluster members (or even outside the cluster) and invite them to take part in the opportunity. }\end{array}$ \\
\hline $\begin{array}{l}\text { R.9. } \\
\text { Secure } \\
\text { partner }\end{array}$ & $\begin{array}{l}\text { - Depending on the type of project, a local partner may just be NZ Embassy staff, or simply } \\
\text { somebody in charge of providing administrative coordination and support. } \\
\text { - In other instances, the local partner is commercial company who is going to be part of the team } \\
\text { that will deliver the service/product (e.g. EENZ has teamed up with an engineering firm in } \\
\text { Istanbul, Turkey). } \\
\text { - Since the EOI only provides a broad scope of the work, there is no assurance that the local partner } \\
\text { would actually be in the team that is going to tender for the project if they get short-listed. The } \\
\text { lead company would re-visit this decision later on. }\end{array}$ \\
\hline $\begin{array}{l}\text { R.10. } \\
\text { Prepare EOI } \\
\text { proposal }\end{array}$ & $\begin{array}{l}\text { - The cluster has developed quick and cost-effective ways to prepare EOIs based on the growing } \\
\text { trust between members and their experience with previous bids. } \\
\text { - The collaborative experience of preparing several EOIs has led to the creation of templates and } \\
\text { case study material that can be adapted for subsequent bids. } \\
\text { - Co-ordination to assemble the EOI does not always fall entirely on the leading company. Another } \\
\text { interested party may offer to help collect the material from the various team members (such as } \\
\text { CVs and track record) and put it together for the EOI. Thus allowing the lead company to focus on } \\
\text { fine-tuning the final EOI, making it look professional and consistent. } \\
\text { - The lead company is responsible for producing the final EOI document that will be shipped away. }\end{array}$ \\
\hline
\end{tabular}




\begin{tabular}{|c|c|}
\hline Activity & Description \\
\hline $\begin{array}{l}\text { R.11. } \\
\text { Delivery of } \\
\text { EOI }\end{array}$ & $\begin{array}{l}\text { - Sending via courier the material that cannot be emailed (such as folders, hard covers, images and } \\
\text { graphics) in advance to local partner. } \\
\text { - Arrange who is actually going to deliver the EOI (e.g. local partner). } \\
\text { - Send electronically the rest of the material to local partner for download. The local partner will put } \\
\text { together all the material from New Zealand, plus their contribution into the agreed EOI. } \\
\text { - In other instances, an electronic version of the EOI is accepted to meet the deadline; however, a } \\
\text { printed document should arrive from New Zealand by courier days later. }\end{array}$ \\
\hline $\begin{array}{l}\text { R.12. } \\
\text { Follow-up } \\
\text { EOI }\end{array}$ & $\begin{array}{l}\text { - The team would not approach the client directly as it would not look professional. If the short- } \\
\text { listing is taking longer (usually over six months), the lead company would politely approach the } \\
\text { client and enquire about the timing of the decision making. } \\
\text { - The cluster however, will informally make a series of actions such as: site visit (if staff of a cluster } \\
\text { member happens to be visiting the particular market) work closely with local partners and } \\
\text { contacts, and counting on the local NZTE Commissioner to check for developments. All this is } \\
\text { fine, as long as they don't try to represent the lead company. }\end{array}$ \\
\hline $\begin{array}{l}\text { R.13. } \\
\text { Short-listed }\end{array}$ & $\begin{array}{l}\text { - It may take some time before hearing anything from the clients. It is common that this could take } \\
\text { up to } 3 \text { months, but there have been cases that the client took up to } 18 \text { months to reply. } \\
\text { - The shorter the short-list is, the more serious the bid is going to be taken. World Bank usually } \\
\text { short-lists a small number of candidates - around six -, which are geographically distributed. } \\
\text { - Client notifies the lead company that their EOI is being accepted to continue the tender process. } \\
\text { They expect a reply from the short-listed candidates soon after. } \\
\text { - Client provides a comprehensive document with the specifications for the tender/final proposal } \\
\text { stage. They would also provide the details on how the proposals will be scored. } \\
\text { - From this point onwards, the cluster involvement drops and a commercial-led approach is } \\
\text { followed where the lead company will be in charge of the bidding process. }\end{array}$ \\
\hline $\begin{array}{l}\text { R.14. } \\
\text { Decide } \\
\text { whether to } \\
\text { continue bid }\end{array}$ & $\begin{array}{l}\text { - Full tender is a very expensive exercise (e.g. tens of thousands of dollars) and therefore careful } \\
\text { consideration by the leader and the team in regards to the viability of a full tender bid. } \\
\text { - When successful in the short-listing process, the companies meet to decide whether or not to } \\
\text { proceed to full tender. They will revisit the issue of whether the project is going to be profitable } \\
\text { enough to justify the risk and cost of preparing a tender. The team will also assess if it would be } \\
\text { advantageous to win the bid as leverage for follow-on work (i.e. "preferred provider"). } \\
\text { - Thorough examination of the details in the specifications required for tender to make sure it is a } \\
\text { viable job, that they are requesting something reasonable, or that the clients are going in the right } \\
\text { direction in terms of methodology and approach. } \\
\text { - Identify the budget of the project and decide which resources are required to fit it. } \\
\text { - Check who are their competitors and if the group stand a chance of beating them. } \\
\text { - Assess if the companies are comfortable with the level of knowledge they have of the market, the } \\
\text { country, and the people they are going to be dealing with. A decision could be made to send } \\
\text { somebody to do an on-site visit. }\end{array}$ \\
\hline $\begin{array}{l}\text { R.15. } \\
\text { Confirm team } \\
\text { for the bid }\end{array}$ & $\begin{array}{l}\text { - There may be some reshuffling of the original team that was put forward at the EOI stage, based } \\
\text { on the specifications detailed in the bid document (i.e. Terms of Reference). A collaborative } \\
\text { approach has to be followed to ensure a suitable mix of experts in order to win the tender. } \\
\text { - Ultimately, the bid leader would decide the composition of the team as they have seen the } \\
\text { "helicopter view" of the project and have a better idea on how to portrait the New Zealand } \\
\text { expertise to make their bid credible. } \\
\text { - The lead company will contact the additional people - even non-members, if that is the case - and } \\
\text { invite them to be part of the team. } \\
\text { - Signing-up to be part of this team means that members will have to honour their commitment to } \\
\text { do their part if the bid is successful. }\end{array}$ \\
\hline $\begin{array}{l}\text { R.16. } \\
\text { Confirm local } \\
\text { partner }\end{array}$ & $\begin{array}{l}\text { - Local partners are assessed on whether they are the right ones based on the detailed tender } \\
\text { specifications. } \\
\text { - Finalising negotiations with local partners. } \\
\text { - It may require a site visit if this is the first time the team works with them. }\end{array}$ \\
\hline
\end{tabular}




\begin{tabular}{|c|c|}
\hline Activity & Description \\
\hline $\begin{array}{l}\text { R.17. } \\
\text { Site visit }\end{array}$ & $\begin{array}{l}\text { - In the reactive bid process, typically somebody goes if it is a major tender and the group has been } \\
\text { short-listed. } \\
\text { - A decision is made to send somebody over if there is no in-depth knowledge of the area, the } \\
\text { market, the local partners, and the clients. Look at the site of the project, the area, meet the key } \\
\text { decision makers, visit the office who is putting out the tender, and spend time with local partner. } \\
\text { Good knowledge of these factors would not justify a site visit. } \\
\text { - Visit does not take long, only a few days. }\end{array}$ \\
\hline $\begin{array}{l}\text { R.18. } \\
\text { Prepare } \\
\text { tender }\end{array}$ & $\begin{array}{l}\text { - The tender has followed a similar format that sets out: 1) the terms of reference for the project; 2) } \\
\text { the methodology that will be employed; 3) who will be in the team; and 4) budget and financials. } \\
\text { - Proposals are starting to come together quickly as the teams are learning how to do them from } \\
\text { previous experiences. } \\
\text { - The lead company provides instructions to the rest of the team on how they want the information } \\
\text { to build the proposal according to the templates provided by the client. } \\
\text { - It is a very intensive period, usually a four-week process. Sometimes it is even less than that } \\
\text { because of the time differences. All hands on it; people working until early hours of the morning } \\
\text { to get bid done on time. } \\
\text { - Team relies on the expertise of the lead company (and sometimes the cluster facilitator) to prepare } \\
\text { the tender to fit the client's requirements. Ultimately, the head person in the lead company is } \\
\text { responsible for the final tender document. } \\
\text { - It does not carry much of the justifications as to why the client should select them, as they already } \\
\text { have been short-listed. } \\
\text { - The tender that goes to the clients involves two critical components: technical and financial. In the } \\
\text { reactive bid process, these two components are delivered in two separate envelopes. A third } \\
\text { critical component is that the companies involved in the bidding process need to finalise the legal } \\
\text { structure (governance). } \\
\text { - There are situations where the bid - or at least parts of it - is translated into the client's native } \\
\text { language. }\end{array}$ \\
\hline $\begin{array}{l}\text { R.18.a. } \\
\text { Technical } \\
\text { component }\end{array}$ & $\begin{array}{l}\text { - The technical component takes most of the time to prepare and is the biggest component of the } \\
\text { tender. } \\
\text { - Defines the terms of reference for the project, what does the client wants and the group's } \\
\text { understanding of it. } \\
\text { - The team proposes how they are going to approach the project, in other words, the methodology. } \\
\text { - World Bank-type tenders require teams to follow strict guidelines and templates for methodology, } \\
\text { CVs, track record, structure of scope of work, etc. }\end{array}$ \\
\hline $\begin{array}{l}\text { R.18.b. } \\
\text { Financial } \\
\text { component }\end{array}$ & $\begin{array}{l}\text { - The price for the project is defined here. } \\
\text { - Budget, financials, and taxes issues. } \\
\text { - World Bank-type tenders only allow for the information to be presented in a certain way. It is a } \\
\text { matter of filling-in their tables. } \\
\text { - The team must not under-price their services. If the team wants to have extra money to cover } \\
\text { unexpected expenses, they need to factor it into their rates. }\end{array}$ \\
\hline $\begin{array}{l}\text { R.18.c. } \\
\text { Governance } \\
\text { (structure of } \\
\text { partnership) }\end{array}$ & $\begin{array}{l}\text { - Agreement on the governance structure that the group will follow (e.g. sub-contracting to lead } \\
\text { company, consortium, partnership, joint-venture, etc.). } \\
\text { - Exchange of letters with the other consultants where they agree on: the company to be the lead } \\
\text { consultant; the proportion of work that each member will take on; sharing of costs, liability, and } \\
\text { profits. } \\
\text { - It can be a tricky process of finalising the mix of people in the team from the various companies } \\
\text { and the balance between experienced and relatively new human resources. } \\
\text { - On occasions, the importance of this aspect gets underestimated in the excitement of doing work. } \\
\text { - Formalising and recording the governance will wait until the team has been successful in the bid. }\end{array}$ \\
\hline $\begin{array}{l}\text { R.19. } \\
\text { Delivery of } \\
\text { tender }\end{array}$ & $\begin{array}{l}\text { - Arrange who is actually going to collate and deliver the final tender: local partner, NZTE } \\
\text { commissioner, or NZ Embassy staff. } \\
\text { - Sending via courier the material that cannot be emailed (such as folders, hard covers, documents } \\
\text { with original signatures, images, and graphics) in advance to whoever is assigned to collate and } \\
\text { deliver the tender. } \\
\text { - Send electronically the rest of the material for download. } \\
\text { - Local partner provides their contribution into the agreed final tender. }\end{array}$ \\
\hline
\end{tabular}




\begin{tabular}{|c|c|}
\hline Activity & Description \\
\hline $\begin{array}{l}\text { R.20. } \\
\text { Follow-up } \\
\text { tender }\end{array}$ & $\begin{array}{l}\text { It is not good practice to directly approach those who are evaluating the tenders; this could lead to } \\
\text { accusations of trying to influence the tender outcome and, consequently banning the group from } \\
\text { bidding. } \\
\text { - Less direct approaches to check for developments (such as communicating with local partners and } \\
\text { contacts, and counting on the local Trade Commission) could be pursued. }\end{array}$ \\
\hline $\begin{array}{l}\text { R.21. } \\
\text { Tender is } \\
\text { selected }\end{array}$ & $\begin{array}{l}\text { The first envelope that is opened is the technical one. The received tenders are ranked following } \\
\text { pre-specified valuation criteria. An official communication from the client to the winning teams } \\
\text { will be sent telling them of their success in passing the technical criteria. The World Bank is also } \\
\text { notified. } \\
\text { - The clients will invite the successful candidates to attend the opening of the financial envelope, } \\
\text { which will take place in a public meeting. The candidates' prices are read out loud. } \\
\text { - It may take a long time before hearing back from the client with a reply as to whether the bid has } \\
\text { been successful or not. } \\
\text { - If the group is in a close second position, the client keeps them quietly in the loop in case their } \\
\text { negotiations with the successful bidder fail. } \\
\text { - Once the team is notified that they have won the tender, they will revisit the governance aspect to } \\
\text { formalise and record the agreement. } \\
\text { - The team will communicate their success to the rest of the cluster. }\end{array}$ \\
\hline $\begin{array}{l}\text { R.22. } \\
\text { Negotiate } \\
\text { final contract }\end{array}$ & $\begin{array}{l}\text { - The lead company will negotiate the contract with the clients and look after the team's interest. } \\
\text { They will also keep the others on the loop. } \\
\text { - The successful bidder will start a negotiation process to finalise the contract. } \\
\text { - There can be a change of boundaries by the client in terms of requirements. } \\
\text { - Sometimes clients will request changes and offsets them by offering additional work which } \\
\text { doesn't need to go to tender. }\end{array}$ \\
\hline $\begin{array}{l}\text { R.23. } \\
\text { Project starts }\end{array}$ & $\begin{array}{l}\text { - Delivery of services and/or products starts. } \\
\text { - The local partner may do some preparatory work while the New Zealand team gears up for the } \\
\text { project. Some team members would need to gradually be taken out of the jobs they are currently } \\
\text { doing to start this new project. } \\
\text { - This is not the end of the process; it is the beginning of a new cycle of identifying new } \\
\text { opportunities that may spinout from this project. }\end{array}$ \\
\hline $\begin{array}{l}\text { R.24. } \\
\text { Project } \\
\text { finishes }\end{array}$ & - Completion of the particular project in the diagrams. \\
\hline $\begin{array}{l}\text { R.25. } \\
\text { Building a } \\
\text { relationship }\end{array}$ & $\begin{array}{l}\text { - This is a key activity because whoever wins a project is very likely to gain extra work in the area } \\
\text { just because the clients already know them. } \\
\text { - Through relationships, new job opportunities are communicated. } \\
\text { - Relationships are built by having people on the ground working on a project as they interact } \\
\text { directly with the client. } \\
\text { - Relationships are also built by making regular visits to the markets of interest. } \\
\text { - Relationships with local partners may lead to additional jobs. } \\
\text { - The clusters are a good vehicle for developing relationships. They have organised meetings with } \\
\text { key opinion leaders and decision makers from SOPAC, Red Cross International, Ministry of } \\
\text { Foreign Affairs, and the World Bank. They have also contributed towards the cost of marketing } \\
\text { visits of cluster members to key countries. } \\
\text { - The clusters have been involved in official visits of delegates from Turkey and China. }\end{array}$ \\
\hline $\begin{array}{l}\text { R.26. } \\
\text { Discussion of } \\
\text { other needs } \\
\text { with clients }\end{array}$ & $\begin{array}{l}\text { - Discussions may take place while the team is on site working on a project. Any additional work } \\
\text { below US } \$ 100,000 \text { in World Bank-type tenders can be negotiated, not tendered. } \\
\text { - Discussions with prospective clients begin about their needs and come up with a proposal tailored } \\
\text { to meet those needs. }\end{array}$ \\
\hline
\end{tabular}




\section{Appendix D: \\ Proactive project bidding process for EENZ and NHNZ Description of the activities involved during the process.}

\begin{tabular}{|c|c|}
\hline$y$ & \\
\hline $\begin{array}{l}\text { P.1. } \\
\text { Building a } \\
\text { relationship }\end{array}$ & $\begin{array}{l}\text { - This is a key activity because whoever wins a project is very likely to gain extra work in the area just } \\
\text { because the clients already know them. } \\
\text { - Through relationships, new job opportunities are communicated. } \\
\text { - Relationships are built by having people on the ground working on a project as they interact directly } \\
\text { with the client. } \\
\text { - Relationships are also built by making regular visits to the markets of interest. } \\
\text { - Relationships with local partners may lead to additional jobs. } \\
\text { - The clusters are a good vehicle for developing relationships. They have organised meetings with key } \\
\text { opinion leaders and decision makers from SOPAC, Red Cross International, Ministry of Foreign } \\
\text { Affairs, and the World Bank. They have also contributed towards the cost of marketing visits of cluster } \\
\text { members to key countries. } \\
\text { - The clusters have been involved in official visits of delegates from Turkey and China. } \\
\text { - Distribution of brochures, company profiles, CVs, project sheets, clusters briefing papers, etc. }\end{array}$ \\
\hline $\begin{array}{l}\text { P.2. } \\
\text { Discuss other } \\
\text { needs with } \\
\text { clients }\end{array}$ & $\begin{array}{l}\text { - Discussions with prospective clients begin about their needs. } \\
\text { - They are usually smaller projects. However, they are attractive not only because they do not go to open } \\
\text { tender but also because there are less risk involved and no significant start-up costs. } \\
\text { - There is a better understanding of what the client wants, which could be reflected in a tailored solution } \\
\text { and a cheaper price for the client. } \\
\text { - The cluster member believes that there could be an opportunity to involve other members. }\end{array}$ \\
\hline $\begin{array}{l}\text { P.3. } \\
\text { Cluster is } \\
\text { alerted }\end{array}$ & $\begin{array}{l}\text { - Is an informal process; any member can make the alert call about the opportunity. The cluster member } \\
\text { notifies the facilitator, PWB, any of the cluster co-chairs, and bigger players in the cluster. } \\
\text { - Those who had identified the opportunity may opt to broadcast it openly to the rest of the cluster, or } \\
\text { may choose to privately approach other members and propose to work together. }\end{array}$ \\
\hline $\begin{array}{l}\text { P.4. } \\
\text { Informal } \\
\text { networking of } \\
\text { interested } \\
\text { members }\end{array}$ & $\begin{array}{l}\text { - Contact other cluster members in an informal manner to find out if there is interest in looking at the } \\
\text { new opportunity. } \\
\text { - Usually the cluster member who is prepared to lead the bid would initiate the networking. } \\
\text { - The interested parties agree to meet to discuss the viability of the project a bit further. }\end{array}$ \\
\hline $\begin{array}{l}\text { P.5. } \\
\text { First meeting } \\
\text { to discuss the } \\
\text { viability of } \\
\text { the } \\
\text { opportunity }\end{array}$ & $\begin{array}{l}\text { - One of the interested parties will call for a meeting at their location (usually it is the cluster member } \\
\text { who is prepared to lead the bid). } \\
\text { - Discuss the project. Questions that may arise are: Does it sound like a real opportunity? Do we have } \\
\text { companies that are excited about this project? Do we have a chance of winning it? } \\
\text { - Some initial assessment of the project's risks, rewards, and whether the cluster has the capability and } \\
\text { capacity required for the project. } \\
\text { - Initial look at who could be the potential team, who may be interested in co-ordinating the putting } \\
\text { together of the proposal, and even some thinking on the type of methodology to approach the } \\
\text { opportunity. }\end{array}$ \\
\hline
\end{tabular}




\begin{tabular}{|c|c|}
\hline Activity & Description \\
\hline $\begin{array}{l}\text { P.6. } \\
\text { Research } \\
\text { further details } \\
\text { of the } \\
\text { opportunity }\end{array}$ & $\begin{array}{l}\text { - This step could be a cascade of further interactions with various bits of information being brought back } \\
\text { and shared. } \\
\text { - Scope out a bit more detail based on the action points defined in the first meeting. } \\
\text { - Try to get more information about the project by emailing and phoning the client and some contacts in } \\
\text { the country. Contacts may be the local NZTE Commission, local partners and known government } \\
\text { officials. } \\
\text { - A site visit may take place. } \\
\text { - Identify the budget of the project and decide which resources are required to fit it. This may affect the } \\
\text { composition of the team. }\end{array}$ \\
\hline $\begin{array}{l}\text { P.7. } \\
\text { Decide } \\
\text { whether to } \\
\text { continue bid }\end{array}$ & $\begin{array}{l}\text { - The companies meet to decide whether or not to proceed to write a proposal for this opportunity. They } \\
\text { will revisit the issue of whether the project is going to be profitable enough to justify the cost of } \\
\text { preparing a proposal. The team will also assess if it would be advantageous to win the bid as leverage } \\
\text { for follow-on work (i.e. preferred provider"). } \\
\text { - Thorough examination of the details in the specifications required by the client, the cluster members } \\
\text { make sure it is a viable job, that they are requesting something reasonable, or that the clients are going } \\
\text { in the right direction in terms of methodology and approach. } \\
\text { - Assess if the companies are comfortable with the level of knowledge they have of the market, the } \\
\text { country, and the people they are going to be dealing with. A decision could be made to send somebody } \\
\text { to do an on-site visit. }\end{array}$ \\
\hline $\begin{array}{l}\text { P.8. } \\
\text { Confirm } \\
\text { member who } \\
\text { will lead } \\
\text { proposal }\end{array}$ & $\begin{array}{l}\text { - Sometimes it is straightforward step: the company who identified the opportunity offers to lead the } \\
\text { proposal since the beginning of the process. Other times it is not clear who will lead the group, but } \\
\text { eventually this is sorted out. } \\
\text { - It is based on overseas connections and a track record. The lead company needs to have a high degree } \\
\text { of sophistication and experience in negotiation in the international arena. It also needs to have some } \\
\text { kind of professional indemnity insurance. } \\
\text { - Leading the proposal is a commitment from the company. This because a huge proportion of the } \\
\text { client's decision to accept the proposal is based on the qualities of the lead company. }\end{array}$ \\
\hline $\begin{array}{l}\text { P.9. } \\
\text { Confirm team } \\
\text { for the } \\
\text { proposal }\end{array}$ & $\begin{array}{l}\text { - It is a self-selecting process. Each individual analyses the opportunity and reflects on whether they have } \\
\text { something to contribute to the project It is a self-selecting process. Each individual analyses the } \\
\text { opportunity and reflects on whether they have something to contribute to the project. It is more through } \\
\text { attrition, or acknowledgement by all parties of who the best team of people is, rather than going through } \\
\text { a formal selection process. } \\
\text { - It could happen that there is a lot of interest from various members with similar skills and expertise and } \\
\text { therefore it would come down to CVs. Usually the situation is the opposite though: the few who are } \\
\text { interested in the project at the beginning realise that they need the expertise of other cluster members } \\
\text { (or even outside the cluster) and invite them to take part in the opportunity. } \\
\text { - Ultimately, the bid leader would decide the composition of the team as they have seen the "helicopter } \\
\text { view" of the project and have a better idea of how to portrait the New Zealand expertise to make the } \\
\text { proposal/bid credible. } \\
\text { - The lead company will contact the additional people - even non-members, if that is the case - and } \\
\text { invite them to be part of the team. } \\
\text { - Other reasons to look for more people to build the team are: the project is much bigger (than first } \\
\text { thought; key people in their companies that could have been involved in this project are already } \\
\text { committed to other jobs; or the initial team does not possess the particular skills for the project or for } \\
\text { that particular market. } \\
\text { - Usually for these projects there is a general idea of its budget and this may affect the composition of the } \\
\text { team. Some initial players may have to accept a minor role in order to keep the proposal's feasibility } \\
\text { attractive to the client. } \\
\text { - Signing-up to be part of this team means that members will have to honour their commitment to do their } \\
\text { part if the proposal is successful. }\end{array}$ \\
\hline
\end{tabular}




\begin{tabular}{|c|c|}
\hline Activity & Description \\
\hline $\begin{array}{l}\text { P.10. } \\
\text { Confirm local } \\
\text { partner }\end{array}$ & $\begin{array}{l}\text { - Considering some potential local partners. } \\
\text { - Depending on the type of project, a local partner may just be NZ Embassy staff, or somebody in charge } \\
\text { of providing administrative coordination and support. In other instances, the local partner is commercial } \\
\text { company who is going to be part of the team that will deliver the service/product. } \\
\text { - Local partners are assessed on whether they are the right ones based on the specifications by the client. } \\
\text { - It may require a visit if this is the first time the team works with them. } \\
\text { - Finalising negotiations with local partners. }\end{array}$ \\
\hline $\begin{array}{l}\text { P.11. } \\
\text { Site visit }\end{array}$ & $\begin{array}{l}\text { - In the proactive bid process, a visit to the site will be programmed if the group feels that there are } \\
\text { strong chances of getting the project. } \\
\text { - A decision is made to send somebody over if there is no in-deep knowledge of the area, the market, the } \\
\text { local partners, and the clients. Look at the site of the project, the area, meet the key decision makers, } \\
\text { visit the office who is putting out the tender, and spend time with local partner. Good knowledge of } \\
\text { these factors would not justify a site visit. } \\
\text { - Visit does not take long, only a few days. }\end{array}$ \\
\hline $\begin{array}{l}\text { P.12. } \\
\text { Prepare } \\
\text { proposal }\end{array}$ & $\begin{array}{l}\text { - In both, the reactive and the proactive bid processes, the proposal/tender has followed a similar format } \\
\text { that sets out: 1) the terms of reference for the project; 2) the methodology that will be employed; 3) who } \\
\text { will be in the team; and 4) budget and financials. } \\
\text { - Deadlines to submit proposals are not as rigid as those from World Bank-type tenders. } \\
\text { - Team relies on the expertise of the facilitator and the lead company to prepare the proposal to fit the } \\
\text { client's requirements. } \\
\text { - The cluster has developed quick and fast ways to prepare proposals based on their experience in } \\
\text { previous bids. These proposals tend to follow a similar format to tenders submitted to World Bank; } \\
\text { however, some variations may apply in order to fulfil the client's requirements. } \\
\text { - There is constant negotiation with the clients, so the proposal evolves over time in terms of cost and } \\
\text { scope. } \\
\text { - The lead company provides instructions to the rest of the team on how they want the information to } \\
\text { build the proposal according to what has been negotiated with the client. } \\
\text { - Co-ordination to assemble the proposal not always falls entirely on the leading company. Another } \\
\text { interested party may offer to help putting together the material. Thus allowing the lead company to } \\
\text { focus in fine-tuning the final proposal, making it look professional and consistent. } \\
\text { - The proposal that goes to the clients involves two critical components in the bid: technical and } \\
\text { financial. A third critical component is that the companies involved in the bidding process need to } \\
\text { finalise the legal structure (governance). } \\
\text { - There are situations where the proposal - or at least parts of it - is translated into the client's native } \\
\text { language. }\end{array}$ \\
\hline $\begin{array}{l}\text { P.12.a. } \\
\text { Technical } \\
\text { proposal }\end{array}$ & $\begin{array}{l}\text { - The technical component takes most of the time to prepare and is the biggest component of the } \\
\text { proposal. } \\
\text { - Defines the terms of reference for the project, what does the client wants and the group's understanding } \\
\text { of it. } \\
\text { - The team proposes how they are going to approach the project, in other words, the methodology. } \\
\text { - Other elements: details of the team }(\mathrm{CVs}) \text {, track record material. }\end{array}$ \\
\hline $\begin{array}{l}\text { P.12.b. } \\
\text { Financial } \\
\text { proposal }\end{array}$ & $\begin{array}{l}\text { - The price for the project is defined here. } \\
\text { - Budget, financials, and taxes issues. }\end{array}$ \\
\hline
\end{tabular}




\begin{tabular}{|c|c|}
\hline Activity & Description \\
\hline $\begin{array}{l}\text { P.12.c. } \\
\text { Governance } \\
\text { (structure of } \\
\text { partnership) }\end{array}$ & $\begin{array}{l}\text { - Agreement on the governance structure that the group will follow (e.g. sub-contracting to lead } \\
\text { company, consortium, partnership, joint-venture, etc.). } \\
\text { - Exchange of letters with the other consultants where they agree on: the company to be the lead } \\
\text { consultant; the proportion of work that each member will take on; sharing of costs, liability, and profits. } \\
\text { - It can be a tricky process of finalising the mix of people in the team from the various companies and the } \\
\text { balance between experienced and relatively new human resources. } \\
\text { - On occasions, the importance of this aspect gets underestimated in the excitement of doing work. } \\
\text { - Formalising and recording the governance will wait until the team has been successful with the } \\
\text { proposal. }\end{array}$ \\
\hline $\begin{array}{l}\text { P.13. } \\
\text { Delivery of } \\
\text { proposal }\end{array}$ & $\begin{array}{l}\text { - Arrange who is actually going to collate and deliver the final tender: local partner, NZTE commissioner, or } \\
\text { NZ Embassy staff. } \\
\text { - Sending via courier the material that cannot be emailed (such as folders, hard covers, documents with original } \\
\text { signatures, images, and graphics) in advance to whoever was assigned to collate and deliver the proposal. } \\
\text { - Send electronically the rest of the material to local partner for download. } \\
\text { - Local partner provides their contribution into the agreed final tender. }\end{array}$ \\
\hline $\begin{array}{l}\text { P.14. } \\
\text { Follow-up } \\
\text { proposal }\end{array}$ & $\begin{array}{l}\text { - In the proactive bid approach, follow-up is more open as a veil of secrecy and diplomacy does not cover the } \\
\text { selection of the project. The client is working directly with the bidding team. } \\
\text { - Make sure that a series of things are been done to encourage the proposal, such as: site visit (if staff of a } \\
\text { cluster member happens to be visiting the particular market) to check for developments, and keep in touch } \\
\text { with the clients. } \\
\text { - Work closely with local partner, as they can call and see the client more often. } \\
\text { - Counting on the local Trade Commission to be knocking at the client's doors, having coffee and finding how } \\
\text { selection process is developing. }\end{array}$ \\
\hline $\begin{array}{l}\text { P.15. } \\
\text { Proposal is } \\
\text { accepted }\end{array}$ & $\begin{array}{l}\text { - It may take a long time before hearing back from the client with a reply as to whether the bid has been } \\
\text { successful or not. } \\
\text { - Once the team is notified that their proposal has been accepted, they will revisit the governance aspect to } \\
\text { formalise and record the agreement. } \\
\text { - The team will communicate their success to the rest of the cluster. }\end{array}$ \\
\hline $\begin{array}{l}\text { P.16. } \\
\text { Negotiate } \\
\text { final contract }\end{array}$ & $\begin{array}{l}\text { - The will start a negotiation process to finalise the contract. } \\
\text { - There can be a change of boundaries by the client in terms of requirements. } \\
\text { - Sometimes clients will request changes and offsets them by offering additional work which doesn't need to go } \\
\text { to tender. }\end{array}$ \\
\hline $\begin{array}{l}\text { P.17. } \\
\text { Project starts }\end{array}$ & $\begin{array}{l}\text { - Delivery of services and/or products starts. } \\
\text { - The local partner may do some preparatory work while the New Zealand team gears up for the project. Some } \\
\text { team members would need to gradually be taken out of the jobs they are currently doing to start this new } \\
\text { project. } \\
\text { - This is not the end of the process; it is the beginning of a new cycle of identifying new opportunities that may } \\
\text { spinout from this project. }\end{array}$ \\
\hline $\begin{array}{l}\text { P.18. Project } \\
\text { finishes }\end{array}$ & f the particular project in the diagrams. \\
\hline
\end{tabular}




\begin{tabular}{|c|c|}
\hline Activity & Description \\
\hline $\begin{array}{l}\text { P.19. } \\
\text { Search and } \\
\text { identify new } \\
\text { opportunities } \\
\text { funded by } \\
\text { World Bank, } \\
\text { ADB, or } \\
\text { similar } \\
\text { institutions. }\end{array}$ & $\begin{array}{l}\text { - World Bank and Asian Development Bank (ADB) expressions of interest are not usually a surprise } \\
\text { when the cluster members are keeping a close eye on what is going on in their markets of interest. } \\
\text { - World Bank first does assessment project reports. After that they will set up the procurement authority } \\
\text { (if one is not already in place), which will be calling for expressions of interest (EOI) for consultants } \\
\text { who wish to make the procurement bids. After that the work for consultants will emerge. } \\
\text { - NHNZ and EENZ pay an annual fee to New Zealand Trade \& Enterprise (NZTE) for World Bank and } \\
\text { ADB Monitoring Liaison Services. } \\
\text { - NZTE tracks World Bank and ADB assessment reports, news releases and procurement notices of what } \\
\text { they are planning to do around the world and sends messages to Positively Wellington Business (PWB). } \\
\text { PWB will then circulate these notices through a distribution list. } \\
\text { - Clusters will also use their contacts in those countries to gain some knowledge about what kind of } \\
\text { projects might soon be announced. } \\
\text { - There is a space during cluster meetings (Marketing Initiatives) where members talk about specific } \\
\text { markets, World Bank and ADB activities. }\end{array}$ \\
\hline
\end{tabular}




\section{Appendix E: \\ Genre Systems Analysis for EENZ-NHNZ Project Bidding}

Table E.1. Genres in the sub-systems R.1 \& P.19 "Search and track new opportunities funded by World Bank, ADB, etc."

\begin{tabular}{|c|c|c|c|c|c|c|}
\hline Genre & Purpose & Content & Actors (i) & Form & Time & Place \\
\hline $\begin{array}{c}\text { Cluster meeting - Marketing } \\
\text { initiatives }\end{array}$ & $\begin{array}{l}\text { Communicate to members } \\
\text { what's going on in specific } \\
\text { target markets (e.g. Iran, } \\
\text { India, Turkey, the Pacific, } \\
\text { etc.), or development } \\
\text { agency (World Bank, ADB, } \\
\text { SOPAC, NZAID, etc.) }\end{array}$ & $\begin{array}{l}\text { Name of } \\
\text { country/organisation, name } \\
\text { of project, names of cluster } \\
\text { members who are involved, } \\
\text { name of contacts, } \\
\text { description of current } \\
\text { situation. }\end{array}$ & $\begin{array}{l}\text { Cluster Co-chair, cluster } \\
\text { members, cluster Facilitator }\end{array}$ & $\begin{array}{l}\text { Medium: Face to face } \\
\text { meeting, telephone } \\
\text { conferencing. Structure: } \\
\text { Structured, brief progress } \\
\text { report. Linguistics: } \\
\text { Informal, conversational. }\end{array}$ & $\begin{array}{l}\text { As part of the cluster } \\
\text { meeting, approx. 5-10 } \\
\text { minutes each specific } \\
\text { country or organisation. }\end{array}$ & $\begin{array}{l}\text { Usually at Positively } \\
\text { Wellington Business (PWB) }\end{array}$ \\
\hline Meeting with key contacts & $\begin{array}{l}\text { Meet with key contacts in } \\
\text { the country/organisation of } \\
\text { interest to gain further } \\
\text { details about what kind of } \\
\text { projects might soon be out } \\
\text { for tender. }\end{array}$ & $\begin{array}{l}\text { Ask for details/updates } \\
\text { about potential } \\
\text { opportunities. Promote the } \\
\text { cluster and its members. }\end{array}$ & $\begin{array}{l}\text { Cluster member or cluster } \\
\text { Facilitator, key contact } \\
\text { (NZTE commissioner, NZ } \\
\text { Embassy official, local } \\
\text { partner, World Bank/ADB } \\
\text { official, colleagues, etc.) }\end{array}$ & $\begin{array}{l}\text { Medium: Face-to-face. } \\
\text { Structure: Greetings, } \\
\text { request details/updates } \\
\text { about potential } \\
\text { opportunities, farewell. } \\
\text { Linguistics: Professional } \\
\text { conversation. }\end{array}$ & Same time. & Same place. \\
\hline Call to track opportunity & $\begin{array}{l}\text { Call key contacts in the } \\
\text { country/organisation of } \\
\text { interest to gain further } \\
\text { details about what kind of } \\
\text { projects might soon be out } \\
\text { for tender. }\end{array}$ & $\begin{array}{l}\text { Ask for details/updates } \\
\text { about potential } \\
\text { opportunities. }\end{array}$ & $\begin{array}{l}\text { Cluster member or cluster } \\
\text { Facilitator, key contact } \\
\text { (NZTE commissioner, NZ } \\
\text { Embassy official, local } \\
\text { partner, World Bank/ADB } \\
\text { official, colleagues, etc.) }\end{array}$ & $\begin{array}{l}\text { Medium: Telephone. } \\
\text { Structure: Greetings, } \\
\text { request details/updates } \\
\text { about potential } \\
\text { opportunities, farewell. } \\
\text { Linguistics: Professional, } \\
\text { conversation. }\end{array}$ & Same time. & Different places. \\
\hline $\begin{array}{l}\text { Message to track } \\
\text { opportunity }\end{array}$ & $\begin{array}{l}\text { Write to key contacts in the } \\
\text { country/organisation of } \\
\text { interest to gain further } \\
\text { details about what kind of } \\
\text { projects might soon be out } \\
\text { for tender. }\end{array}$ & $\begin{array}{l}\text { Ask for details/updates } \\
\text { about potential } \\
\text { opportunities. }\end{array}$ & $\begin{array}{l}\text { Cluster member or cluster } \\
\text { Facilitator, key contact } \\
\text { (NZTE commissioner, NZ } \\
\text { Embassy official, local } \\
\text { partner, World Bank/ADB } \\
\text { official, colleagues, etc.) }\end{array}$ & $\begin{array}{l}\text { Medium: Email. } \\
\text { Structure: Greetings, } \\
\text { request details/updates } \\
\text { about potential } \\
\text { opportunities, farewell. } \\
\text { Linguistics: Informal but } \\
\text { professional. }\end{array}$ & Any time. & Any place. Outlook. \\
\hline $\begin{array}{l}\text { World Bank extracts of } \\
\text { news (iNTE Monitoring } \\
\text { Liaison Notices) }\end{array}$ & $\begin{array}{l}\text { Inform interested parties } \\
\text { about economic } \\
\text { development in various } \\
\text { regions in the world (e.g. } \\
\text { India, Iraq, the Caribbean, } \\
\text { etc). To inform World Bank } \\
\text { funding activities in certain } \\
\text { regions (e.g. plans/approval } \\
\text { to lend money to a } \\
\text { particular country). }\end{array}$ & $\begin{array}{l}\text { Subject, body (extract of the } \\
\text { news), name of the Trade } \\
\text { Comissioner who collated } \\
\text { the information, } \\
\text { Commissioner's contact } \\
\text { details, disclaimer notice. }\end{array}$ & $\begin{array}{l}\text { Trade Commissioner in } \\
\text { Washington, PWB official, } \\
\text { cluster members. }\end{array}$ & $\begin{array}{l}\text { Medium: Email (distribution } \\
\text { list) Structure: Formal, } \\
\text { paragraphs of the news } \\
\text { extract and then the name } \\
\text { and contact details of the } \\
\text { NZ Trade Commissioner in } \\
\text { Washington, a disclaimer. } \\
\text { Linguistics: Formal, } \\
\text { reporting style. }\end{array}$ & View at any time. & Any place. Outlook. \\
\hline $\begin{array}{l}\text { World Bank News Release } \\
\text { (NZTE Monitoring Liaison } \\
\text { Notices) }\end{array}$ & $\begin{array}{l}\text { To inform interested parties } \\
\text { about World Bank funding } \\
\text { activities. }\end{array}$ & $\begin{array}{l}\text { Title, news about the } \\
\text { approval of credit for } \\
\text { various projects around the } \\
\text { world. }\end{array}$ & $\begin{array}{l}\text { World Bank officials, } \\
\text { NZTE officials in } \\
\text { Washington, PWB Liaison, } \\
\text { cluster members. }\end{array}$ & $\begin{array}{l}\text { Medium: Email (distribution } \\
\text { list), sometimes with links to } \\
\text { the WB website. } \\
\text { Structure: Formal, news } \\
\text { release. Linguistics: } \\
\text { Formal reporting style. }\end{array}$ & View at any time. & $\begin{array}{l}\text { WB Website, Outlook. Any } \\
\text { place. }\end{array}$ \\
\hline $\begin{array}{l}\text { What's new at the InfoShop } \\
\text { (World Bank) (NZTE } \\
\text { Monitoring Liaison Notices) }\end{array}$ & $\begin{array}{l}\text { Provide the URL links to } \\
\text { different information } \\
\text { available at the World } \\
\text { Bank's Public Information } \\
\text { Centre. }\end{array}$ & $\begin{array}{l}\text { Message from the NZTE } \\
\text { Washington Office } \\
\text { instructing how to access } \\
\text { the specific information. } \\
\text { Week of the year. Then a } \\
\text { content table linking to } \\
\text { Webpages for the specific } \\
\text { type of document assorted } \\
\text { by country: Project } \\
\text { Appraisal Document, } \\
\text { Country Financial } \\
\text { Accountability Assessment, } \\
\text { Implimentation Completion } \\
\text { Reports, Sector Reports, } \\
\text { Project Information } \\
\text { Dcouments, Chairman's } \\
\text { Concluding Remarks, } \\
\text { Integrated Safeguards Data } \\
\text { Sheets, etc. (NOTE: each } \\
\text { instance may not have all } \\
\text { these titles, as it would } \\
\text { depend on whether there is } \\
\text { something to report in that } \\
\text { particular week). }\end{array}$ & $\begin{array}{l}\text { New Zealand Trade and } \\
\text { Enterprise Washington } \\
\text { Office, PWB Liaison, } \\
\text { cluster members. }\end{array}$ & $\begin{array}{l}\text { Medium: Email (distribution } \\
\text { list) with links to specific } \\
\text { documents in the WB } \\
\text { website. Structure: } \\
\text { Formal, a table of content. } \\
\text { Linguistics: Formal but } \\
\text { brief, it is a list of topics. }\end{array}$ & $\begin{array}{l}\text { Weekly posts, view at any } \\
\text { time within that frame. }\end{array}$ & $\begin{array}{l}\text { World Bank Website, } \\
\text { Outlook. Any place. }\end{array}$ \\
\hline
\end{tabular}

(i) The highlighted actors are the ones who usually initiate the genre. 
Table E.1. Genres in the sub-systems R.1 \& P.19 "Search and track new opportunities funded by World Bank, ADB, etc." (Cont.).

\begin{tabular}{|c|c|c|c|c|c|c|}
\hline Genre & Purpose & Content & Actors (i) & Form & Time & Place \\
\hline $\begin{array}{l}\text { Project Information } \\
\text { Document (World Bank) } \\
\text { (NZTE Monitoring Liaison } \\
\text { Notices) }\end{array}$ & $\begin{array}{l}\text { Describe projects that the } \\
\text { World Bank is considering } \\
\text { to fund. }\end{array}$ & $\begin{array}{l}\text { Title of type of document, } \\
\text { stage of development } \\
\text { (conceptual, appraisal). } \\
\text { A table that holds key, } \\
\text { info: project name, region, } \\
\text { sector, project ID, } \\
\text { Eorrowers, Implementing } \\
\text { Agency, environment } \\
\text { category, safeguard } \\
\text { classification, Date PID } \\
\text { prepared, (estimated) } \\
\text { date of appraisal, Date of } \\
\text { board approval. Then } \\
\text { document has sections } \\
\text { regarding: Country and } \\
\text { sector background, } \\
\text { objectives, rationale for } \\
\text { Eank involvernent, } \\
\text { description, financing, } \\
\text { implementation, sateguard } \\
\text { policies that might apply, } \\
\text { etc. }\end{array}$ & $\begin{array}{l}\text { World Bank officials, } \\
\text { NZTE officials in } \\
\text { Washington, PMB official, } \\
\text { cluster members. } \\
\end{array}$ & $\begin{array}{l}\text { Medium: Email } \\
\text { (distribution list), the } \\
\text { report is a PDF attached } \\
\text { to the ernail. Structure: } \\
\text { Formal report; there is a } \\
\text { table with the key details } \\
\text { such as name of project, } \\
\text { country and project ID. } \\
\text { The report is divided in } \\
\text { pre-determined headings. } \\
\text { Linguistics: Formal } \\
\text { report. }\end{array}$ & $\begin{array}{l}\text { 3-6 months; it varies. } \\
\text { View at any time within } \\
\text { that frame. }\end{array}$ & $\begin{array}{l}\text { World Bank Website, } \\
\text { Outlook. Any place }\end{array}$ \\
\hline $\begin{array}{l}\text { New projects from } \\
\text { Monthly Operational } \\
\text { Summary (MB) }\end{array}$ & $\begin{array}{l}\text { Notify new projects listed } \\
\text { in the monthly operational } \\
\text { summary. }\end{array}$ & $\begin{array}{l}\text { Extracts from the Monthly } \\
\text { Operational Sumnary. } \\
\text { Divided by country and by } \\
\text { sector. One paragraph } \\
\text { which sumnarises the } \\
\text { objective of the project, } \\
\text { the stage where the } \\
\text { project is (e.g. pre- } \\
\text { appraisal, appraisal, } \\
\text { appraisal completed), } \\
\text { funding granted to the } \\
\text { project, if it would require } \\
\text { consultants, } \\
\text { environmental } \\
\text { assessment category, } \\
\text { and contact details. }\end{array}$ & $\begin{array}{l}\text { IZTE officials in } \\
\text { Washington, PWB } \\
\text { Liaison, cluster members. }\end{array}$ & $\begin{array}{l}\text { Medium: Email } \\
\text { (distribution list). } \\
\text { Structure: Formal } \\
\text { summaries per country } \\
\text { and sector. The new } \\
\text { projects are structured in } \\
\text { a pre-determined manner. } \\
\text { Linguistics: Formal } \\
\text { report. }\end{array}$ & View at any time. & $\begin{array}{l}\text { World Bank Website, } \\
\text { Outlook. }\end{array}$ \\
\hline $\begin{array}{l}\text { Procurement Notices } \\
\text { (World Bank) } \\
\text { (NZTE Monitoring Liaison } \\
\text { Notices) }\end{array}$ & $\begin{array}{l}\text { Invite eligible consultants } \\
\text { to indicate their interest in } \\
\text { carrying out the project. }\end{array}$ & $\begin{array}{l}\text { Title: Procurement Notice. } \\
\text { Then there is a set of key } \\
\text { information listed: } \\
\text { country, project, } \\
\text { financing, abstract, } \\
\text { sector, loanicredit } \\
\text { number, contract/bid } \\
\text { number, and deadline. } \\
\text { A.ter that a one-page } \\
\text { summarny of: context of } \\
\text { the project, objectives, } \\
\text { key activities and eligibility } \\
\text { of bidders. Finally the } \\
\text { contact details where the } \\
\text { EOl should be submitted. }\end{array}$ & $\begin{array}{l}\text { World Bank Officials, } \\
\text { funding recipient (client), } \\
\text { NZTE Washington Office, } \\
\text { FME Liaison, cluster } \\
\text { members. }\end{array}$ & $\begin{array}{l}\text { Medium: Email } \\
\text { (distribution list). } \\
\text { Structure: } \text { Highly } \\
\text { structured. There is a } \\
\text { table with the key details } \\
\text { such as name of project, } \\
\text { country and project ID. } \\
\text { The report is divided in } \\
\text { pre-determined headings. } \\
\text { Linguistics: Formal } \\
\text { contract. }\end{array}$ & $\begin{array}{l}\text { Up to } 3 \text { weeks, but } \\
\text { usually less than that. } \\
\text { View at any time within } \\
\text { that frame. }\end{array}$ & $\begin{array}{l}\text { World Bank Website, } \\
\text { Outlook. Any place. }\end{array}$ \\
\hline $\begin{array}{l}\text { ADE News Release } \\
\text { (NZTE Monitoring Liaison } \\
\text { Notices) }\end{array}$ & $\begin{array}{l}\text { Notify recipients of links } \\
\text { to news releases by the } \\
\text { A.DB. }\end{array}$ & $\begin{array}{l}\text { A list of titles with links to } \\
\text { the actual news at the } \\
\text { ADB Website. }\end{array}$ & $\begin{array}{l}\text { IIZTE Philippines } \\
\text { Office, PME Liaison, } \\
\text { cluster members. }\end{array}$ & $\begin{array}{l}\text { Medium: Email } \\
\text { (distribution list) with links } \\
\text { to specific news releases } \\
\text { in the ADE website. } \\
\text { Structure: Formal but } \\
\text { brief, like a table of } \\
\text { contents. } \\
\text { Linguistics: Formal but } \\
\text { brief, it is a list of news } \\
\text { headlines. } \\
\end{array}$ & $\begin{array}{l}\text { Monthly. View at any } \\
\text { time within that frame. }\end{array}$ & $\begin{array}{l}\text { ADE Website, Outlook. } \\
\text { Any place. }\end{array}$ \\
\hline $\begin{array}{l}\text { ADE New Project Lists } \\
\text { (NZTE Monitoring Liaison } \\
\text { Notices) }\end{array}$ & $\begin{array}{l}\text { Provide the URL links to } \\
\text { new projects listed in the } \\
\text { A.DE's Website. }\end{array}$ & $\begin{array}{l}\text { Title with the date, } \\
\text { headings of various fields } \\
\text { such as Agriculture and } \\
\text { Natural Rresources, } \\
\text { Energy, Social } \\
\text { Infrastructure, Finance, } \\
\text { Others, etc. Under each } \\
\text { heading there is a list of } \\
\text { projects by country and a } \\
\text { link to ADE's Website. }\end{array}$ & $\begin{array}{l}\text { ADB officials, NZTE } \\
\text { Philippines Office, PWV } \\
\text { Liaison, cluster members. }\end{array}$ & $\begin{array}{l}\text { Medium: Email } \\
\text { (distribution list) with links } \\
\text { to specific documents in } \\
\text { the ADE website. } \\
\text { Structure: Formal, a } \\
\text { table of content. } \\
\text { Linguistics: Formal but } \\
\text { briet, it is a list of topics. }\end{array}$ & $\begin{array}{l}\text { Fortnightly. View at any } \\
\text { time within that frame. }\end{array}$ & $\begin{array}{l}\text { ADE Website, Outlook. } \\
\text { Any place. }\end{array}$ \\
\hline $\begin{array}{l}\text { ADE Publication Lists } \\
\text { (NZTE Monitoring Liaison } \\
\text { Notices) }\end{array}$ & $\begin{array}{l}\text { Provide a list of the latest } \\
\text { ADE documents that are } \\
\text { available in pdf format, so } \\
\text { cluster members can } \\
\text { request specific ones via } \\
\text { enail. }\end{array}$ & $\begin{array}{l}\text { Greetings, body, contact } \\
\text { details of NZTE official in } \\
\text { Philippines. The } \\
\text { attachment has a table of } \\
\text { three columns: ADB } \\
\text { documents available in } \\
\text { pdf, Dcoument type and } \\
\text { country. }\end{array}$ & $\begin{array}{l}\text { IIZTE Philippines } \\
\text { office, PWE Liaison, } \\
\text { cluster members. }\end{array}$ & $\begin{array}{l}\text { Medium: Email } \\
\text { (distribution list) with an } \\
\text { attachment to a document } \\
\text { listing the ADB documents } \\
\text { now available. } \\
\text { Structure: Formal, } \\
\text { follows a letter format; } \\
\text { the attachement } \\
\text { document is a list of } \\
\text { documents (sometimes } \\
\text { inside a table, others just } \\
\text { a list). Linguistics: } \\
\text { Formal but brief letter, the } \\
\text { attachment is a list of } \\
\text { documents. } \\
\end{array}$ & $\begin{array}{l}\text { Once-twice a month. } \\
\text { View at any time within } \\
\text { that frame. }\end{array}$ & Outlook. Any place. \\
\hline
\end{tabular}


Table E.2. Genres in the sub-system R.2 "Expression of Interest (EOI) is announced."

\begin{tabular}{|c|c|c|c|c|c|c|}
\hline Genre & Purpose & Content & Actors (i) & Form & Time & Place \\
\hline $\begin{array}{c}\text { Procurement Notice (World } \\
\text { Bank) }\end{array}$ & $\begin{array}{l}\text { Invite eligible consultants to } \\
\text { register their interest in } \\
\text { carrying out the project. } \\
\text { List what the EOI should } \\
\text { include. }\end{array}$ & $\begin{array}{l}\text { Title: Procurement Notice. } \\
\text { Then there is a set of key } \\
\text { information listed in a table: } \\
\text { country, project, financing, } \\
\text { abstract, sector, loan/credit } \\
\text { number, contract/bid } \\
\text { number, and deadline. } \\
\text { After that, there is a one- } \\
\text { page summmary of: context } \\
\text { of the project, objectives, } \\
\text { key activities and eligibility } \\
\text { of bidders. Finally, } \\
\text { specifications of what the } \\
\text { EOI should include and the } \\
\text { contact details where the } \\
\text { EOI should be submitted } \\
\text { and its deadline. }\end{array}$ & $\begin{array}{l}\text { World Bank, the funding } \\
\text { recipient (client), NZTE } \\
\text { Washington Office, PWB } \\
\text { Liaison, cluster members. }\end{array}$ & $\begin{array}{l}\text { Medium: Email (distribution } \\
\text { list). Structure: Formal } \\
\text { contractual there is a table } \\
\text { with the key details such as } \\
\text { name of project, country } \\
\text { and project ID. The } \\
\text { procurement notice is } \\
\text { divided in pre-determined } \\
\text { headings. Linguistics: } \\
\text { Formal contract. }\end{array}$ & $\begin{array}{l}\text { Up to } 3 \text { weeks, but usually } \\
\text { less than that. View at any } \\
\text { time within that frame. }\end{array}$ & $\begin{array}{l}\text { World Bank Website, } \\
\text { Outlook. Any place. }\end{array}$ \\
\hline $\begin{array}{l}\text { ADB New Project Lists } \\
\text { (NZTE Monitoring Liaison } \\
\text { Notices) }\end{array}$ & $\begin{array}{l}\text { Provide the URL links to } \\
\text { new projects listed in the } \\
\text { ADB's Website. }\end{array}$ & $\begin{array}{l}\text { Title with the date, headings } \\
\text { of various fields such as } \\
\text { Agriculture and Natural } \\
\text { Rresources, Energy, Social } \\
\text { Infrastructure, Finance, } \\
\text { Others, etc. Under each } \\
\text { heading there is a list of } \\
\text { projects by country and a } \\
\text { link to ADB's Website. }\end{array}$ & $\begin{array}{l}\text { ADB officials, NZTE } \\
\text { Philippines Office, PWB } \\
\text { Liaison, cluster members. }\end{array}$ & $\begin{array}{l}\text { Medium: Email (distribution } \\
\text { list) with links to specific } \\
\text { documents in the ADB } \\
\text { website. Structure: } \\
\text { Formal, a table of contents. } \\
\text { Linguistics: Formal but } \\
\text { brief, it is a list of topics. }\end{array}$ & $\begin{array}{l}\text { Fortnightly. View at any } \\
\text { time within that frame. }\end{array}$ & $\begin{array}{l}\text { ADB Website, Outlook. } \\
\text { Any place. }\end{array}$ \\
\hline $\begin{array}{c}\text { Request for Expressions of } \\
\text { Interest }\end{array}$ & $\begin{array}{l}\text { Invite eligible consultants to } \\
\text { register their interest in } \\
\text { carrying out the project. } \\
\text { List what the EOI should } \\
\text { contain. }\end{array}$ & $\begin{array}{l}\text { Title of the project. } \\
\text { Summary of: context of the } \\
\text { project, objectives, key } \\
\text { activities and details of } \\
\text { bidders' eligibility. Finally, } \\
\text { specifications of what the } \\
\text { EOI should include and the } \\
\text { contact details where the } \\
\text { EOI should be submitted } \\
\text { and its deadline. }\end{array}$ & $\begin{array}{l}\text { The client, NZTE } \\
\text { commissioner, PWB } \\
\text { Liaison, cluster members. }\end{array}$ & $\begin{array}{l}\text { Medium: Email (distribution } \\
\text { list) with attachment. } \\
\text { Structure: Formal } \\
\text { contractual The request is } \\
\text { divided in pre-determined } \\
\text { headings. Linguistics: } \\
\text { Formal contract. }\end{array}$ & $\begin{array}{l}\text { Up to } 3 \text { weeks, but usually } \\
\text { less than that. View at any } \\
\text { time within that frame. }\end{array}$ & Outlook. Any place. \\
\hline
\end{tabular}

Table E.3. Genres in the sub-system R.3 \& P.3 "Cluster is alerted."

\begin{tabular}{|c|c|c|c|c|c|c|}
\hline Genre & Purpose & Content & Actors (i) & Form & Time & Place \\
\hline Alert Message Reactive & $\begin{array}{l}\text { Let other members know } \\
\text { about the opportunity. }\end{array}$ & $\begin{array}{l}\text { Forwarding the procurement } \\
\text { notice with a brief message } \\
\text { indicating the potential fit for } \\
\text { the types of services offered } \\
\text { by other cluster members } \\
\text { and point out your own } \\
\text { interest. }\end{array}$ & $\begin{array}{l}\text { Cluster member, } \\
\text { Facilitator, cluster Co- } \\
\text { chairs, PWB Liaison. }\end{array}$ & $\begin{array}{l}\text { Medium: Email, usually } \\
\text { forwarding the Procurement } \\
\text { Notice. Structure: A } \\
\text { greeting, body of message } \\
\text { indicating the reason for } \\
\text { writing, and then an } \\
\text { attachement of procurement } \\
\text { notice. Linguistics: } \\
\text { Informal announcement, } \\
\text { professional. }\end{array}$ & $\begin{array}{l}\text { Urgent, within the } \\
\text { submission's deadline. Any } \\
\text { time within that frame. }\end{array}$ & Outlook. Any place. \\
\hline Alert Call Reactive & $\begin{array}{l}\text { Let other members know } \\
\text { about the opportunity. }\end{array}$ & $\begin{array}{l}\text { Indicate the potential fit for } \\
\text { the types of services offered } \\
\text { by other cluster members } \\
\text { and point out your own } \\
\text { interest. Reference to the } \\
\text { specific procurement notice. }\end{array}$ & $\begin{array}{l}\text { Cluster member, } \\
\text { Facilitator, cluster Co- } \\
\text { chairs, PWB Liaison. }\end{array}$ & $\begin{array}{l}\text { Medium: Telephone call. } \\
\text { Structure: Greetings, } \\
\text { reasons for calling, and } \\
\text { farewell. } \quad \text { Linguistics: } \\
\text { Informal but professional. }\end{array}$ & $\begin{array}{l}\text { Urgent, within the } \\
\text { submission's deadline. Any } \\
\text { time within that frame. }\end{array}$ & $\begin{array}{l}\text { Telephone lines. Member's } \\
\text { office or mobile phone. Any } \\
\text { place. }\end{array}$ \\
\hline Alert Message Proactive & $\begin{array}{l}\text { Let other members know } \\
\text { about the opportunity. } \\
\text { Show interest in pursuing } \\
\text { this opportunity. }\end{array}$ & \begin{tabular}{|l|} 
Brief message indicating \\
the potential opportunity. \\
Indicate the potential fit for \\
the types of services offered \\
by other cluster members \\
and point out your own \\
interest.
\end{tabular} & $\begin{array}{l}\text { Cluster member, } \\
\text { Facilitator, cluster Co- } \\
\text { chairs, PWB Liaison. }\end{array}$ & \begin{tabular}{|l|} 
Medium: Email. \\
Structure: A greeting, body \\
of message indicating the \\
reason for writing, and then \\
an attachement of \\
procurement notice. \\
Linguistics: Informal \\
announcement, \\
professional.
\end{tabular} & $\begin{array}{l}\text { Within the submission's } \\
\text { deadline. Any time within } \\
\text { that frame. }\end{array}$ & Outlook. Any place. \\
\hline Alert Call Proactive & $\begin{array}{l}\text { Let other members know } \\
\text { about the opportunity. } \\
\text { Show interest in pursuing } \\
\text { this opportunity. }\end{array}$ & $\begin{array}{l}\text { Indicate the potential fit for } \\
\text { the types of services offered } \\
\text { by other cluster members } \\
\text { and point out your own } \\
\text { interest. }\end{array}$ & $\begin{array}{l}\text { Cluster member, } \\
\text { Facilitator, cluster Co- } \\
\text { chairs, PWB Liaison. }\end{array}$ & $\begin{array}{l}\text { Medium: Telephone call. } \\
\text { Structure: Greetings, } \\
\text { reasons for calling, and } \\
\text { farewell. Linguistics: } \\
\text { Informal but professional. }\end{array}$ & $\begin{array}{l}\text { Within the submission's } \\
\text { deadline. Any time within } \\
\text { that frame. }\end{array}$ & $\begin{array}{l}\text { Telephone lines. Member's } \\
\text { office or mobile phone. Any } \\
\text { place. }\end{array}$ \\
\hline
\end{tabular}


Table E.4. Genres in the sub-system R.4 \& P.4 "Informal networking of interested members."

\begin{tabular}{|c|c|c|c|c|c|c|}
\hline Genre & Purpose & Content & Actors (i) & Form & Time & Place \\
\hline Networking Memo & $\begin{array}{l}\text { Invite members to explore } \\
\text { the opportunity further. Find } \\
\text { out if there is interest in } \\
\text { pursuing the opportunity. }\end{array}$ & $\begin{array}{l}\text { Memo indicating that } \\
\text { one/several company/ies } \\
\text { is/are interested in } \\
\text { submitting an EOI. Inviting } \\
\text { others to contact key people } \\
\text { to explore this potential } \\
\text { collaborative bid. }\end{array}$ & $\begin{array}{l}\text { Cluster Facilitator, PWB } \\
\text { Liaison, interested cluster } \\
\text { members. }\end{array}$ & $\begin{array}{l}\text { Medium: Email (usually } \\
\text { forwarding the Procurement } \\
\text { Notice). Structure: A } \\
\text { greeting, body of message } \\
\text { indicating the business of } \\
\text { the message, a farewell, } \\
\text { contact details, and then an } \\
\text { attachement of procurement } \\
\text { notice. Linguistics: } \\
\text { Informal announcement, } \\
\text { professional. }\end{array}$ & $\begin{array}{l}\text { Urgent, within the } \\
\text { submission's deadline. Any } \\
\text { time within that frame. }\end{array}$ & Outlook. Any place. \\
\hline Networking message & $\begin{array}{l}\text { Reply the Networking } \\
\text { Memo. }\end{array}$ & $\begin{array}{l}\text { Member informs his/her } \\
\text { interest in exploring the } \\
\text { opportunity further. }\end{array}$ & $\begin{array}{l}\text { Interested cluster } \\
\text { member, Facilitator, PWB } \\
\text { Liaison, potential lead } \\
\text { company. }\end{array}$ & $\begin{array}{l}\text { Medium: Email. } \\
\text { Structure: A greeting, body } \\
\text { of message indicating } \\
\text { interest in exploring } \\
\text { opportunity further, a } \\
\text { farewell, contact details. } \\
\text { Linguistics: Informal but } \\
\text { professional. }\end{array}$ & $\begin{array}{l}\text { Urgent, within the } \\
\text { submission's deadline. Any } \\
\text { time within that frame. }\end{array}$ & Outlook. Any place. \\
\hline Networking calls & $\begin{array}{l}\text { Discuss with members the } \\
\text { opportunity further. Find } \\
\text { out if there is interest in } \\
\text { pursuing the opportunity. } \\
\text { Organise a meeting. }\end{array}$ & $\begin{array}{l}\text { Discussing and inquiring } \\
\text { further information about } \\
\text { the opportunity. Details } \\
\text { about the member's } \\
\text { potential fit for the types of } \\
\text { services required. Agreeing } \\
\text { on a time and place to } \\
\text { meet. }\end{array}$ & $\begin{array}{l}\text { Potential lead company, } \\
\text { cluster member, } \\
\text { Facilitator. }\end{array}$ & $\begin{array}{l}\text { Medium: Telephone call. } \\
\text { Structure: Greetings, } \\
\text { reasons for calling, and } \\
\text { farewell. Linguistics: } \\
\text { Informal but professional. }\end{array}$ & $\begin{array}{l}\text { Urgent, within the } \\
\text { submission's deadline. Any } \\
\text { time within that frame. }\end{array}$ & Telephone. Any place. \\
\hline
\end{tabular}

Table E.5. Genres in the sub-system R.5 \& P.5 "First meeting: the viability of the opportunity".

\begin{tabular}{|c|c|c|c|c|c|c|}
\hline Genre & Purpose & Content & Actors (i) & Form & Time & Place \\
\hline Meeting invitation & $\begin{array}{l}\text { Invite interested members } \\
\text { to attend First Meeting. }\end{array}$ & $\begin{array}{l}\text { Salutation, details about } \\
\text { opportunity, time, place and } \\
\text { date of meeting. }\end{array}$ & $\begin{array}{l}\text { Potential lead company, } \\
\text { cluster members interested } \\
\text { in the opportunity, cluster } \\
\text { Facilitator. }\end{array}$ & $\begin{array}{l}\text { Medium: Email. } \\
\text { Structure: A greeting, body } \\
\text { of message indicating } \\
\text { details about the meeting, a } \\
\text { farewell. Linguistics: } \\
\text { Informal but professional. }\end{array}$ & $\begin{array}{l}\text { Urgent, within the } \\
\text { submission's deadline. Any } \\
\text { time within that frame. }\end{array}$ & Outlook. Any place. \\
\hline $\begin{array}{l}\text { First Meeting- all members } \\
\text { in same location }\end{array}$ & $\begin{array}{l}\text { Discuss the viability of the } \\
\text { opportunity. Decide } \\
\text { whether or not to continue } \\
\text { the bid process. }\end{array}$ & $\begin{array}{l}\text { Discussion by interested } \\
\text { parties regarding the } \\
\text { project's risks, rewards, and } \\
\text { whether or not the cluster } \\
\text { has the capability and } \\
\text { capacity required for the } \\
\text { project. Initial look at who } \\
\text { could be the potential lead } \\
\text { company and bidding team, } \\
\text { who may be interested in co } \\
\text { ordinating the putting } \\
\text { together of the EOI } \\
\text { (reactive) or proposal } \\
\text { (proactive), and even some } \\
\text { thinking on the type of } \\
\text { methodology to approach } \\
\text { the opportunity. }\end{array}$ & $\begin{array}{l}\text { Potential lead company, } \\
\text { cluster members interested } \\
\text { in the opportunity, cluster } \\
\text { Facilitator. }\end{array}$ & \begin{tabular}{|l|} 
Medium: Face to face \\
meeting, or telephone \\
conference (national \& \\
international). \\
Structure: There is not a \\
strict order, but the meeting \\
must cover risks, rewards, \\
who will co-ordinate the EOI \\
(reactive) or proposal \\
(proactive), who will lead \\
the bidd, type of \\
methodology, and a set of \\
action points to be assigned \\
to attendants. \\
Linguistics: Informal, \\
conversational; some \\
technical and contractual \\
vocabulary.
\end{tabular} & $\begin{array}{l}\text { One or two hours. Same } \\
\text { time. }\end{array}$ & $\begin{array}{l}\text { At one of the interested } \\
\text { parties' premises, same } \\
\text { place. With phone } \\
\text { conferencing, in different } \\
\text { places. }\end{array}$ \\
\hline Action Points & \begin{tabular}{|l|} 
List the set of activities that \\
need to be done and assign \\
a person to look after \\
specific points.
\end{tabular} & $\begin{array}{l}\text { List of actions or tasks that } \\
\text { need to be carried out to } \\
\text { find more information about } \\
\text { the opportunity., }\end{array}$ & $\begin{array}{l}\text { Potential lead company, } \\
\text { cluster members } \\
\text { interested in the } \\
\text { opportunity, cluster } \\
\text { Facilitator. }\end{array}$ & $\begin{array}{l}\text { Medium: Whiteboard, } \\
\text { personal notes. } \\
\text { Structure: There is not a } \\
\text { strict order, list of action } \\
\text { points and the name of the } \\
\text { person who has beeen } \\
\text { assigned the task. } \\
\text { Linguistics: Casual. }\end{array}$ & $\begin{array}{l}\text { Any time within the frame } \\
\text { agreed by the group. }\end{array}$ & Any place. \\
\hline
\end{tabular}


Table E.6. Genres in the sub-system R.6 \& P.6 "Research further details of the opportunity."

\begin{tabular}{|c|c|c|c|c|c|c|}
\hline Genre & Purpose & Content & Actors (i) & Form & Time & Place \\
\hline Meeting with key contacts & $\begin{array}{l}\text { Meet with key contacts in } \\
\text { the country/organisation of } \\
\text { interest to gain further } \\
\text { details about the nature of } \\
\text { the project. }\end{array}$ & $\begin{array}{l}\text { Ask for details, background } \\
\text { informartion about the } \\
\text { potential opportunity. }\end{array}$ & \begin{tabular}{|l|} 
Cluster member \\
interested in the \\
opportunity, key contact \\
(client, NZTE \\
Commissioner, NZ \\
Embassy official, local \\
partner, World Bank/ADB \\
official, colleagues, etc.)
\end{tabular} & $\begin{array}{l}\text { Medium: Face-to-face. } \\
\text { Structure: Greetings, } \\
\text { request details/updates } \\
\text { about potential } \\
\text { opportunities, farewell. } \\
\text { Linguistics: Professional } \\
\text { conversation. }\end{array}$ & Same place. & Same time. \\
\hline Research Further Calls & $\begin{array}{l}\text { Scope out more details } \\
\text { about the opportunity based } \\
\text { on the action points to avoid } \\
\text { commiting further time and } \\
\text { resources to an unfeasible } \\
\text { project. }\end{array}$ & $\begin{array}{l}\text { Find about the situation, } \\
\text { who is leading the calling } \\
\text { for bids, and the country. }\end{array}$ & $\begin{array}{l}\text { Cluster members } \\
\text { interested in the } \\
\text { opportunity, cluster } \\
\text { Facilitator, contacts in the } \\
\text { country of the project (e.g. } \\
\text { NZTE officials, embassy } \\
\text { staff, local partners, } \\
\text { colleagues, friends, etc.). }\end{array}$ & $\begin{array}{l}\text { Medium: Telephone. } \\
\text { Structure: Greetings, } \\
\text { reasons for calling, and } \\
\text { farewell. Linguistics: } \\
\text { Informal but professional. }\end{array}$ & $\begin{array}{l}\text { Urgent, within the } \\
\text { submission's deadline. Any } \\
\text { time within that frame. }\end{array}$ & Any place. \\
\hline Research Further Emails & $\begin{array}{l}\text { Scope out more details } \\
\text { about the opportunity based } \\
\text { on the action points to avoid } \\
\text { commiting further time and } \\
\text { resources to an unfeasible } \\
\text { project. }\end{array}$ & $\begin{array}{l}\text { Find about the situation, } \\
\text { who is leading the calling } \\
\text { for bids, and the country. }\end{array}$ & $\begin{array}{l}\text { Cluster members } \\
\text { interested in the } \\
\text { opportunity, cluster } \\
\text { Facilitator, contacts in the } \\
\text { country of the project (e.g. } \\
\text { NZTE officials, embassy } \\
\text { staff, local partners, } \\
\text { colleagues, friends, etc.). }\end{array}$ & $\begin{array}{l}\text { Medium: Email. } \\
\text { Structure: Greetings, } \\
\text { reasons for writing the email } \\
\text { and farewell. Linguistics: } \\
\text { Informal but professional. }\end{array}$ & $\begin{array}{l}\text { Urgent, within the } \\
\text { submission's deadline. Any } \\
\text { time within that frame. }\end{array}$ & Outlook. Any place. \\
\hline Search Results & $\begin{array}{l}\text { Search over the Internet for } \\
\text { any background information } \\
\text { about the country/region } \\
\text { where the project would } \\
\text { take place, and about the } \\
\text { organisation that is calling } \\
\text { for bids. }\end{array}$ & $\begin{array}{l}\text { Descriptions of the } \\
\text { situation, who is leading the } \\
\text { calling for bids, and the } \\
\text { country/region where the } \\
\text { project will take place. }\end{array}$ & $\begin{array}{l}\text { Cluster members } \\
\text { interested in the } \\
\text { opportunity, cluster } \\
\text { Facilitator. }\end{array}$ & $\begin{array}{l}\text { Medium: The Internet } \\
\text { (WWW). Structure: List of } \\
\text { websites with relevant } \\
\text { information; whatever it is } \\
\text { available over the Internet. } \\
\text { Linguistics: Professional. }\end{array}$ & $\begin{array}{l}\text { Urgent, within the } \\
\text { submission's deadline. Any } \\
\text { time within that frame. }\end{array}$ & $\begin{array}{l}\text { The Internet (WWW). Any } \\
\text { place. }\end{array}$ \\
\hline $\begin{array}{l}\text { Report Findings Meeting -all } \\
\text { members in same location }\end{array}$ & $\begin{array}{l}\text { Report of findings to the } \\
\text { rest of the team. }\end{array}$ & $\begin{array}{l}\text { Details of what it has been } \\
\text { found about the project from } \\
\text { contacts and search over } \\
\text { the Internet. }\end{array}$ & $\begin{array}{l}\text { Cluster members } \\
\text { interested in the } \\
\text { opportunity, cluster } \\
\text { Facilitator. }\end{array}$ & $\begin{array}{l}\text { Medium: Face to face } \\
\text { meeting or telephone- } \\
\text { conference. } \\
\text { Structure: Structured, brief } \\
\text { progress report. } \\
\text { Linguistics: Informal, } \\
\text { conversation. }\end{array}$ & $\begin{array}{l}\text { As part of the team } \\
\text { meeting. Same time. }\end{array}$ & $\begin{array}{l}\text { At one of the interested } \\
\text { parties. Same place. If } \\
\text { there is a member away } \\
\text { and they are using phon- } \\
\text { conferencing, then differen } \\
\text { places. }\end{array}$ \\
\hline
\end{tabular}

Table E.7. Genres in the sub-system R.7 \& P.8 "Confirm member who will lead bid."

\begin{tabular}{|c|c|c|c|c|c|c|}
\hline Genre & Purpose & Content & Actors (i) & Form & Time & Place \\
\hline Team Meeting & $\begin{array}{l}\text { Co-ordinate and discuss } \\
\text { viability of opportunity. } \\
\text { Confirm who is going to } \\
\text { lead the bid/proposal. }\end{array}$ & $\begin{array}{l}\text { Discussion of action points. } \\
\text { Acknowledgement by } \\
\text { interested members that a } \\
\text { particular company will lead } \\
\text { the bid/proposal. }\end{array}$ & $\begin{array}{l}\text { Interested cluster } \\
\text { members, cluster } \\
\text { Facilitator. }\end{array}$ & $\begin{array}{l}\text { Medium: Face-to-face. } \\
\text { Structure: Informal, } \\
\text { discussion. Linguistics: } \\
\text { Professional conversation. }\end{array}$ & Same place. & Same time. \\
\hline Confirming Memo & $\begin{array}{l}\text { Inform cluster members } \\
\text { about the team that is being } \\
\text { set up to tackle the new } \\
\text { opportunity and who is } \\
\text { going to lead it. }\end{array}$ & $\begin{array}{l}\text { Memo indicating that a } \\
\text { company will lead the EOI. }\end{array}$ & $\begin{array}{l}\text { Cluster Facilitator, team } \\
\text { leader, PWB Liaison, } \\
\text { cluster members. }\end{array}$ & $\begin{array}{l}\text { Medium: Email. } \\
\text { Structure: A greeting, body } \\
\text { of message indicating the } \\
\text { business of the message, a } \\
\text { farewell, and contact } \\
\text { details. Linguistics: } \\
\text { Informal announcement, } \\
\text { professional. }\end{array}$ & $\begin{array}{l}\text { Within the submission's } \\
\text { deadline. Any time within } \\
\text { that frame. }\end{array}$ & Outlook. Any place. \\
\hline
\end{tabular}


Table E.8. Genres in the sub-system R.8 “Confirm team for EOI."

\begin{tabular}{|c|c|c|c|c|c|c|}
\hline Recruiting Team Call & $\begin{array}{l}\text { Ask additional members (or } \\
\text { non-cluster members) to } \\
\text { join the team. }\end{array}$ & $\begin{array}{l}\text { Mention the project and } \\
\text { indicate the potential fit for } \\
\text { the types of services offered } \\
\text { by the new recruit. Request } \\
\text { for CV. }\end{array}$ & Team leader, new recruit. & $\begin{array}{l}\text { Medium: Telephone call. } \\
\text { Structure: Greetings, } \\
\text { reasons for calling, and } \\
\text { farewell. Language: } \\
\text { Informal but professional. }\end{array}$ & $\begin{array}{l}\text { Urgent, within the } \\
\text { submission's deadline. Any } \\
\text { time within that frame. }\end{array}$ & $\begin{array}{l}\text { Telephone lines. Member's } \\
\text { office or mobile phone. Any } \\
\text { place. }\end{array}$ \\
\hline Recruiting Team Email & $\begin{array}{l}\text { Ask additional members (or } \\
\text { non-cluster members) to } \\
\text { join the team. }\end{array}$ & $\begin{array}{l}\text { Forwarding the procurement } \\
\text { notice with a brief message } \\
\text { indicating that a team is } \\
\text { being formed to prepare an } \\
\text { EOI, show the potential fit } \\
\text { for the types of services that } \\
\text { the new recruit offers, and } \\
\text { invite him/her to join the } \\
\text { team. Request for CV. }\end{array}$ & Team leader, new recruit. & $\begin{array}{l}\text { Medium: Email, usually } \\
\text { forwarding the Procurement } \\
\text { Notice. Structure: A } \\
\text { greeting, body of message } \\
\text { indicating the reason for } \\
\text { writing, and then an } \\
\text { attachement of procurement } \\
\text { notice. Languge: } \\
\text { Informal invitation but } \\
\text { professional. }\end{array}$ & $\begin{array}{l}\text { Urgent, within the } \\
\text { submission's deadline. Any } \\
\text { time within that frame. }\end{array}$ & Outlook. Any place. \\
\hline $\mathrm{CV}$ & $\begin{array}{l}\text { Provide information about } \\
\text { the individual in order to be } \\
\text { considered for a job. To } \\
\text { select the team for the EOI. }\end{array}$ & $\begin{array}{l}\text { Personal information, } \\
\text { education, work experience, } \\
\text { areas of expertise, } \\
\text { experience, capabilities and } \\
\text { contact details. }\end{array}$ & $\begin{array}{l}\text { Candidate team member, } \\
\text { team leader. }\end{array}$ & \begin{tabular}{|l|} 
Medium: Email, usually \\
forwarding the CV. \\
Structure: Highly \\
structured; subheadings for \\
each set of information, \\
such as personal details, \\
education, training, \\
experience, etc. \\
Languge: Formal and \\
professional.
\end{tabular} & $\begin{array}{l}\text { Urgent, within the } \\
\text { submission's deadline. Any } \\
\text { time within that frame. }\end{array}$ & Outlook. Any place. \\
\hline Team Meeting & $\begin{array}{l}\text { Co-ordinate and discuss } \\
\text { who is going to be in the } \\
\text { team for the EOI. }\end{array}$ & $\begin{array}{l}\text { Discussion of who is going } \\
\text { to be part of the team to be } \\
\text { put forward for the EOI. }\end{array}$ & $\begin{array}{l}\text { Interested cluster } \\
\text { members, team leaders, } \\
\text { cluster Facilitator. }\end{array}$ & \begin{tabular}{|l|} 
Medium: Face-to-face. \\
Structure: Informal, \\
discussion. Linguistics: \\
Professional conversation.
\end{tabular} & Same place. & Same time. \\
\hline Team Leader Call & $\begin{array}{l}\text { Inform who is going to be } \\
\text { part of the team. }\end{array}$ & $\begin{array}{l}\text { Communication of who is } \\
\text { going to be part of the team. }\end{array}$ & $\begin{array}{l}\text { Team leader, candidate } \\
\text { team members. }\end{array}$ & $\begin{array}{l}\text { Medium: Telephone call. } \\
\text { Structure: Greetings, } \\
\text { reasons for calling, and } \\
\text { farewell. Linguistics: } \\
\text { Informal but professional. }\end{array}$ & $\begin{array}{l}\text { Urgent, within the } \\
\text { submission's deadline. Any } \\
\text { time within that frame. }\end{array}$ & $\begin{array}{l}\text { Telephone lines. Member's } \\
\text { office or mobile phone. Any } \\
\text { place. }\end{array}$ \\
\hline
\end{tabular}

Table E.9. Genres in the sub-system R.9 "Secure local partner."

\begin{tabular}{|c|c|c|c|c|c|c|}
\hline Genre & Purpose & Content & Actors (i) & Form & Time & Place \\
\hline Meeting with Local Partner & $\begin{array}{l}\text { Meet a potential local } \\
\text { partner and assess how } \\
\text { established, credible and } \\
\text { connected they are. }\end{array}$ & $\begin{array}{l}\text { Talks about their } \\
\text { background, visit to their } \\
\text { premises, showcase their } \\
\text { work, etc. Discussions } \\
\text { about types of projects that } \\
\text { both parties could do jointly. }\end{array}$ & $\begin{array}{l}\text { Team leader, team } \\
\text { member, local partner. }\end{array}$ & $\begin{array}{l}\text { Medium: Face to face } \\
\text { meeting. Structure: There } \\
\text { is not a strict order, but the } \\
\text { meeting must cover } \\
\text { background of the local } \\
\text { partner, its capability and } \\
\text { capacity, previous jobs, } \\
\text { interest in working with the } \\
\text { cluster. Linguistics: } \\
\text { Informal, conversational; } \\
\text { some technical and } \\
\text { business vocabulary. }\end{array}$ & Same time. & $\begin{array}{l}\text { At the local partner's } \\
\text { premises. Same place. }\end{array}$ \\
\hline Message to Local Partner & $\begin{array}{l}\text { Invite eligible local } \\
\text { partner(s) to join the NZ } \\
\text { team who is interested in } \\
\text { carrying out the project. }\end{array}$ & $\begin{array}{l}\text { Forwarding the procurement } \\
\text { notice with a brief message } \\
\text { indicating the potential fit for } \\
\text { the types of services offered } \\
\text { by local partner. }\end{array}$ & $\begin{array}{l}\text { Team leader, cluster } \\
\text { member who knows the } \\
\text { local partner, cluster } \\
\text { Facilitator, local partner. }\end{array}$ & $\begin{array}{l}\text { Medium: Email, usually } \\
\text { forwarding the Procurement } \\
\text { Notice. Structure: A } \\
\text { greeting, body of message } \\
\text { indicating the reason for } \\
\text { writing, and then an } \\
\text { attachment of procurement } \\
\text { notice. Linguistics: } \\
\text { Informal announcement, } \\
\text { professional. }\end{array}$ & $\begin{array}{l}\text { Urgent, within the } \\
\text { submission's deadline. Any } \\
\text { time within that frame. }\end{array}$ & Outlook. Any place. \\
\hline Call to Local Partner & $\begin{array}{l}\text { Invite eligible local } \\
\text { partner(s) interested in } \\
\text { carrying out the project to } \\
\text { join the NZ team. }\end{array}$ & $\begin{array}{l}\text { Indicate the potential fit for } \\
\text { the types of services offered } \\
\text { by the local partner. } \\
\text { Reference to the specific } \\
\text { procurement notice. }\end{array}$ & Team leader, local partner. & $\begin{array}{l}\text { Medium: Telephone call. } \\
\text { Structure: Greetings, } \\
\text { reasons for calling, and } \\
\text { farewell. Linguistics: } \\
\text { Formal and professional. }\end{array}$ & $\begin{array}{l}\text { Urgent, within the } \\
\text { submission's deadline. Any } \\
\text { time within that frame. }\end{array}$ & $\begin{array}{l}\text { Telephone lines. Member's } \\
\text { office or mobile phone. Any } \\
\text { place. }\end{array}$ \\
\hline
\end{tabular}


Table E.10. Genres in the sub-system "Prepare EOI."

\begin{tabular}{|c|c|c|c|c|c|c|}
\hline Genre & Purpose & Content & Actors (i) & Form & Time & Place \\
\hline $\begin{array}{l}\text { Procurement Notices } \\
\text { (Morld Bank) }\end{array}$ & $\begin{array}{l}\text { Invite eligible consultants } \\
\text { to register their interest in } \\
\text { carrying out the project. } \\
\text { List what the EOI should } \\
\text { include. }\end{array}$ & $\begin{array}{l}\text { Title: Procurement Notice. } \\
\text { Then there is a set of key } \\
\text { information listed in a } \\
\text { table: country, project, } \\
\text { financing, abstract, } \\
\text { sector, loan/credit } \\
\text { number, contract/bid } \\
\text { number, and deadline. } \\
\text { After that, there is a one- } \\
\text { page summary of: context } \\
\text { of the project, objectives, } \\
\text { key activities and eligibility } \\
\text { of bidders. Finally, } \\
\text { specifications of what the } \\
\text { EOl should include and } \\
\text { the contact details where } \\
\text { the EOI should be } \\
\text { subritted and its } \\
\text { deadline. }\end{array}$ & $\begin{array}{l}\text { World Bank, the funding } \\
\text { recipient (client), NZTE } \\
\text { Washington Office, PMU } \\
\text { Liaison, cluster members. } \\
\end{array}$ & $\begin{array}{l}\text { Medium: Email } \\
\text { (distribution list). } \\
\text { Structure: Formal } \\
\text { contractual there is a } \\
\text { table with the key details } \\
\text { such as name of project, } \\
\text { country and project ID. } \\
\text { The procurement notice is } \\
\text { divided into pre- } \\
\text { deternined headings. } \\
\text { Linguistics: Formal } \\
\text { contract. }\end{array}$ & $\begin{array}{l}\text { Up to } 3 \text { weeks, but } \\
\text { usually less than that. } \\
\text { View at any time within } \\
\text { that frame. }\end{array}$ & $\begin{array}{l}\text { World Eank Website, } \\
\text { Outlook. Any place. }\end{array}$ \\
\hline $\begin{array}{c}\text { Request for Expressions } \\
\text { of Interest }\end{array}$ & $\begin{array}{l}\text { Invite eligible consultants } \\
\text { to register their interest in } \\
\text { carrying out the project. } \\
\text { List what the EOI should } \\
\text { include. }\end{array}$ & $\begin{array}{l}\text { Title of the project. } \\
\text { Summary of: context of } \\
\text { the project, objectives, } \\
\text { key activities and details } \\
\text { of bidders' eligibility. } \\
\text { Finally, specifications of } \\
\text { what the EOI should } \\
\text { include and the contact } \\
\text { details where the EOI } \\
\text { should be submitted and } \\
\text { its deadline. }\end{array}$ & $\begin{array}{l}\text { The client, NZTE } \\
\text { commissioner, PME } \\
\text { Liaison, cluster members. }\end{array}$ & $\begin{array}{l}\text { Medium: Email } \\
\text { (distribution list) with } \\
\text { attachment. } \\
\text { Structure: Formal } \\
\text { contractual The request } \\
\text { is divided in pre- } \\
\text { determined headings. } \\
\text { Linguistics: Formal } \\
\text { contract. }\end{array}$ & $\begin{array}{l}\text { Up to } 3 \text { weeks, but } \\
\text { usually less than that. } \\
\text { View at any time within } \\
\text { that frame. }\end{array}$ & Outlook. Any place. \\
\hline Prepare EOI Meeting & $\begin{array}{l}\text { Coordinate the assembly } \\
\text { of the EOl. }\end{array}$ & $\begin{array}{l}\text { Discussion about what to } \\
\text { include in the EOI, } \\
\text { regarding methodology, } \\
\text { CVs and requests for } \\
\text { material that needs to be } \\
\text { part of the EOI. }\end{array}$ & $\begin{array}{l}\text { Team leader, EOI } \\
\text { Coor dinator, rest of EOI } \\
\text { team, cluster Facilitator. }\end{array}$ & $\begin{array}{l}\text { Medium: Face to face } \\
\text { meeting, telephone } \\
\text { conference. } \\
\text { Structure: Structured, } \\
\text { follows an agenda set by } \\
\text { the team leader or the EOI } \\
\text { Co-ordinator. } \\
\text { Linguistics: Informal, } \\
\text { conversation. }\end{array}$ & $\begin{array}{l}\text { Urgent, within the } \\
\text { submission's deadline. } \\
\text { Same time within that } \\
\text { frame. }\end{array}$ & $\begin{array}{l}\text { At one of the interested } \\
\text { parties' prennises. Same } \\
\text { place. If telephone- } \\
\text { conferencing is being } \\
\text { used, then different } \\
\text { places. }\end{array}$ \\
\hline Request Call & $\begin{array}{l}\text { Request tearn members } \\
\text { for further information to } \\
\text { complete the EOI } \\
\text { document. }\end{array}$ & $\begin{array}{l}\text { Greetings, request } \\
\text { specific information } \\
\text { farewell. }\end{array}$ & $\begin{array}{l}\text { Cluster member who is } \\
\text { part of the bidding team, } \\
\text { team leader, cluster } \\
\text { Faciltator. }\end{array}$ & $\begin{array}{l}\text { Medium: Telephone } \\
\text { conference. } \\
\text { Structure: Formal } \\
\text { business meeting. } \\
\text { Linguistics: Formal, } \\
\text { business-oriented. }\end{array}$ & Same time. & $\begin{array}{l}\text { Telephone line. Different } \\
\text { place. }\end{array}$ \\
\hline Reply Message & $\begin{array}{l}\text { Reply to request. Provide } \\
\text { the additional information } \\
\text { needed to complete the } \\
\text { EOI document. }\end{array}$ & $\begin{array}{l}\text { Greetings, submission of } \\
\text { specific information, } \\
\text { farewell. }\end{array}$ & $\begin{array}{l}\text { EOl team member, } \\
\text { tearn leader. }\end{array}$ & $\begin{array}{l}\text { Medium: Email, usually } \\
\text { with key information } \\
\text { attached. } \\
\text { Structure: Greetings, } \\
\text { reasons for writing the } \\
\text { email and farewell. } \\
\text { Language: Informal but } \\
\text { professional. }\end{array}$ & $\begin{array}{l}\text { Urgent, within the } \\
\text { submission's deadline. } \\
\text { Any time within that } \\
\text { frame. }\end{array}$ & Outlook. Any place. \\
\hline Expert Profile & $\begin{array}{l}\text { Provide information about } \\
\text { the expert. }\end{array}$ & $\begin{array}{l}\text { Information about the } \\
\text { expert's contact details, } \\
\text { work history, record of } \\
\text { work in similar projects, } \\
\text { education and training. }\end{array}$ & $\begin{array}{l}\text { Team leader, EOI team, } \\
\text { cluster Facilitator. }\end{array}$ & $\begin{array}{l}\text { Medium: Document; it is } \\
\text { usually distributed via } \\
\text { email. Structure: Very } \\
\text { structured, it follows pre- } \\
\text { determined headings. } \\
\text { Linguistics: Formal, } \\
\text { business-oriented. }\end{array}$ & $\begin{array}{l}\text { Urgent, within the } \\
\text { submission's deadline. } \\
\text { Any time within that } \\
\text { frame. }\end{array}$ & Any place. \\
\hline $\begin{array}{l}\text { Project Sheet } \\
\text { (Track Record) }\end{array}$ & $\begin{array}{l}\text { Provide track record of } \\
\text { previous jobs done by } \\
\text { cluster members who are } \\
\text { part of the EOI tearn. }\end{array}$ & $\begin{array}{l}\text { Information about work in } \\
\text { previous projects with } \\
\text { similar characteristics. } \\
\text { Some details are: project } \\
\text { name, client, value of } \\
\text { project, description of } \\
\text { project, specific tasks } \\
\text { performed in the project. }\end{array}$ & $\begin{array}{l}\text { Team member, team } \\
\text { leader. }\end{array}$ & $\begin{array}{l}\text { Medium: Document; it is } \\
\text { usually distributed via } \\
\text { email. } \\
\text { Structure: Very } \\
\text { structured, it follows a } \\
\text { template. } \\
\text { Linguistics: Formal, } \\
\text { highly technical business- } \\
\text { oriented. } \\
\end{array}$ & $\begin{array}{l}\text { Within the EOl's deadline. } \\
\text { Any time within that } \\
\text { trame. }\end{array}$ & Same place. \\
\hline $\begin{array}{l}\text { Expression of Interest } \\
\text { (EOI) }\end{array}$ & $\begin{array}{l}\text { To be considered for the } \\
\text { tender short-list. } \\
\text { Introduce the tean of } \\
\text { experts and showcase } \\
\text { their work experience on } \\
\text { the field. }\end{array}$ & $\begin{array}{l}\text { Cover page with pictures, } \\
\text { introduction (why use this } \\
\text { group of consultants, our } \\
\text { understanding of the } \\
\text { project), areas of } \\
\text { expertise, companies' } \\
\text { description and } \\
\text { capabilities, records of } \\
\text { previous jobs, list of } \\
\text { experts and their CVs, } \\
\text { contact details, } \\
\text { conclusion. }\end{array}$ & $\begin{array}{l}\text { EOI team, local partner, } \\
\text { client. }\end{array}$ & $\begin{array}{l}\text { Medium: Printed } \\
\text { document, fax, email. } \\
\text { Structure: Very } \\
\text { structured, it follows a } \\
\text { template. Linguistics: } \\
\text { Formal, highly technical } \\
\text { and business-oriented. }\end{array}$ & $\begin{array}{l}\text { Urgent, within the } \\
\text { submission's deadline. } \\
\text { Any time within that } \\
\text { frame. }\end{array}$ & Any place. \\
\hline Company Brochure & $\begin{array}{l}\text { To showcase the } \\
\text { companies who are part } \\
\text { of the team. }\end{array}$ & $\begin{array}{l}\text { Set of glossy material and } \\
\text { brochures describing the } \\
\text { companies and their } \\
\text { capabilities that is } \\
\text { attached to the EOI. }\end{array}$ & $\begin{array}{l}\text { EOl team, local partners, } \\
\text { client. }\end{array}$ & $\begin{array}{l}\text { Medium: Printed } \\
\text { document posted by } \\
\text { courier } \\
\text { Structure: Very } \\
\text { structured, marketing- } \\
\text { type format. } \\
\text { Linguistics: Formal, } \\
\text { technical and } \\
\text { businessinarketing. }\end{array}$ & Any time. & Any place. \\
\hline
\end{tabular}


Table E.11. Genres in the sub-system R.11 "Delivery of EOI."

\begin{tabular}{|c|c|c|c|c|c|c|}
\hline Genre & Purpose & Content & Actors (i) & Form & Time & Place \\
\hline Covers of EOI & $\begin{array}{l}\text { Inform the reader the title of } \\
\text { the document's title and } \\
\text { authors. }\end{array}$ & $\begin{array}{l}\text { Title of the document, } \\
\text { authors, date, pictures and } \\
\text { graphic design. }\end{array}$ & \begin{tabular}{|l} 
Team leader, local \\
partners, client.
\end{tabular} & $\begin{array}{l}\text { Medium: Printed pages } \\
\text { sent via courier. } \\
\text { Structure: Very structured, } \\
\text { it follows a template. } \\
\text { Linguistics: Formal, highly } \\
\text { technical and business- } \\
\text { oriented. }\end{array}$ & $\begin{array}{l}\text { Urgent, within the } \\
\text { submission's deadline. Any } \\
\text { time within that frame. }\end{array}$ & Same place. \\
\hline "Boiler Plate" Material & $\begin{array}{l}\text { To showcase the } \\
\text { companies who are part of } \\
\text { the team. }\end{array}$ & $\begin{array}{l}\text { Set of glossy material and } \\
\text { brochures describing the } \\
\text { companies. }\end{array}$ & $\begin{array}{l}\text { EOI team, local partners, } \\
\text { recipients of EOI }\end{array}$ & $\begin{array}{l}\text { Medium: Printed document } \\
\text { sent via courier. } \\
\text { Structure: Very structured, } \\
\text { brochure-type format. } \\
\text { Linguistics: Formal, } \\
\text { technical and business } \\
\text { oriented. }\end{array}$ & Any time. & Same place. \\
\hline Message with EOI & $\begin{array}{l}\text { Send the electronic version } \\
\text { of the EOI to the local } \\
\text { partners. }\end{array}$ & $\begin{array}{l}\text { Brief message with EOI } \\
\text { document attached. }\end{array}$ & $\begin{array}{l}\text { Team leader, local } \\
\text { partners, recipients of EOI }\end{array}$ & $\begin{array}{l}\text { Medium: Email with EOI } \\
\text { attached. } \\
\text { Structure: Very structured, } \\
\text { it follows a template. } \\
\text { Linguistics: Formal, highly } \\
\text { technical and business- } \\
\text { oriented. }\end{array}$ & $\begin{array}{l}\text { Urgent, within the } \\
\text { submission's deadline. Any } \\
\text { time within that frame. }\end{array}$ & Outlook. Any place. \\
\hline
\end{tabular}

Table E.12. Genres in the sub-system R.12 "Follow-up EOI."

\begin{tabular}{|c|c|c|c|c|c|c|}
\hline Genre & Purpose & Content & Actors (i) & Form & Time & Place \\
\hline Follow-up Meeting & $\begin{array}{l}\text { Find out how the selection } \\
\text { process is coming along. }\end{array}$ & $\begin{array}{l}\text { Informal visits to key } \\
\text { contacts that may have } \\
\text { some knowledge about the } \\
\text { selection process. }\end{array}$ & $\begin{array}{l}\text { Cluster member who is } \\
\text { visiting the market, } \\
\text { cluster facilitator, local } \\
\text { partners, NZTE and } \\
\text { Embassy staff, key } \\
\text { contacts, Government } \\
\text { officials, client. }\end{array}$ & $\begin{array}{l}\text { Medium: Face-to-face } \\
\text { meetings. } \\
\text { Structure: Informal, casual. } \\
\text { Linguistics: Casual, } \\
\text { business-oriented. }\end{array}$ & Same time. & Same place. \\
\hline Follow-up Calls & $\begin{array}{l}\text { Find out how the selection } \\
\text { process is coming along. }\end{array}$ & $\begin{array}{l}\text { Discussing and inquiring } \\
\text { any information about the } \\
\text { progress of the selection } \\
\text { process. }\end{array}$ & $\begin{array}{l}\text { Local partners, NZTE and } \\
\text { Embassy staff, cluster } \\
\text { Facilitator, lead company, } \\
\text { Government officials, client. }\end{array}$ & $\begin{array}{l}\text { Medium: Telephone calls. } \\
\text { Structure: Informal, casual. } \\
\text { Linguistics: Casual, } \\
\text { business-oriented. }\end{array}$ & Same time. & Different place. \\
\hline Follow-up Messages & $\begin{array}{l}\text { Find out how the selection } \\
\text { process is coming along. }\end{array}$ & $\begin{array}{l}\text { Discussing and inquiring } \\
\text { any information about the } \\
\text { progress of the selection } \\
\text { process. }\end{array}$ & $\begin{array}{l}\text { Local partners, NZTE and } \\
\text { Embassy staff, cluster } \\
\text { Facilitator, lead company, } \\
\text { Government officials, client. }\end{array}$ & $\begin{array}{l}\text { Medium: Email. } \\
\text { Structure: Informal, casual. } \\
\text { Linguistics: Casual, } \\
\text { business-oriented. }\end{array}$ & Different time. & Outlook. Different place. \\
\hline
\end{tabular}


Table E.13. Genres in the sub-system R.13 "Short-listed."

\begin{tabular}{|c|c|c|c|c|c|c|}
\hline Genre & Purpose & Content & Actors (i) & Form & Time & Place \\
\hline Notification of Short-list & $\begin{array}{l}\text { Client notifies that the team } \\
\text { has been short-listed. }\end{array}$ & $\begin{array}{l}\text { Greetings, notify that the } \\
\text { team has been shortlisted, } \\
\text { instructions for the next } \\
\text { stage. }\end{array}$ & Client, lead company. & $\begin{array}{l}\text { Medium: Email with Tender } \\
\text { Terms of Reference } \\
\text { attached. Structure: } \\
\text { Greetings, reasons for } \\
\text { writing the email and } \\
\text { farewell. Linguistics: } \\
\text { Formal, contractual. }\end{array}$ & $\begin{array}{l}\text { Within the tender's } \\
\text { deadline. Any time within } \\
\text { that frame. }\end{array}$ & Any place. \\
\hline Tender Terms of Reference & $\begin{array}{l}\text { Instruct what needs to be } \\
\text { submited for the tender } \\
\text { process. }\end{array}$ & $\begin{array}{l}\text { Defined guidelines for the } \\
\text { technical proposal. There } \\
\text { are formats for the CVs and } \\
\text { the strucuture of the report. } \\
\text { Details about the scope of } \\
\text { work, methodology. They } \\
\text { also describe the marking } \\
\text { criteria for the tender. }\end{array}$ & Client, lead company. & $\begin{array}{l}\text { Medium: Document; it is } \\
\text { sent as an attachment via } \\
\text { email. Structure: Highly } \\
\text { structured. Linguistics: } \\
\text { Formal, highly technical and } \\
\text { contractual. }\end{array}$ & $\begin{array}{l}\text { Within the tender's } \\
\text { deadline. Any time within } \\
\text { that frame. }\end{array}$ & Any place. \\
\hline Announcement Call & $\begin{array}{l}\text { Communicate the team } \\
\text { member and the cluster } \\
\text { facilitator that their EOI } \\
\text { application has been } \\
\text { successful. }\end{array}$ & $\begin{array}{l}\text { Greetings, announcing the } \\
\text { good news, indication that } \\
\text { the tender's documentation } \\
\text { will be sent via email to } \\
\text { team members, farewell. }\end{array}$ & $\begin{array}{l}\text { Team leader, cluster } \\
\text { members involved in the } \\
\text { team. }\end{array}$ & $\begin{array}{l}\text { Medium: Telephone call. } \\
\text { Structure: Greetings, } \\
\text { reasons for calling, and } \\
\text { farewell. Linguistics: } \\
\text { Informal but professional. }\end{array}$ & $\begin{array}{l}\text { Within the tender's } \\
\text { deadline. Same time within } \\
\text { that frame. }\end{array}$ & Telephone line. Any place. \\
\hline $\begin{array}{l}\text { Informing the Team } \\
\text { Message }\end{array}$ & $\begin{array}{l}\text { Communicate the team that } \\
\text { their EOI application has } \\
\text { been successful. }\end{array}$ & $\begin{array}{l}\text { Greetings, announcing that } \\
\text { the team has been short- } \\
\text { listed, invitation to read the } \\
\text { tender's documentation that } \\
\text { is attached, farewell. }\end{array}$ & $\begin{array}{l}\text { Team leader, cluster } \\
\text { members involved in the } \\
\text { team. }\end{array}$ & $\begin{array}{l}\text { Medium: Email with Tender } \\
\text { Terms of Reference } \\
\text { attached. Structure: } \\
\text { Greetings, reasons for } \\
\text { writing the email and } \\
\text { farewell. Language: } \\
\text { Informal but professional. }\end{array}$ & $\begin{array}{l}\text { Within the tender's } \\
\text { deadline. Any time within } \\
\text { that frame. }\end{array}$ & Outlook. Any place. \\
\hline Short-list Memo & $\begin{array}{l}\text { Communicate the rest of } \\
\text { the cluster that the EOI } \\
\text { team has been succesful. }\end{array}$ & $\begin{array}{l}\text { Heading with title of the } \\
\text { project. Announcement of } \\
\text { the good news and } \\
\text { informing members that } \\
\text { further details would be } \\
\text { discussed in the next } \\
\text { cluster meeting. }\end{array}$ & $\begin{array}{l}\text { Cluster Facilitator, team } \\
\text { leader, cluster members. }\end{array}$ & \begin{tabular}{|l|} 
Medium: Email. \\
Structure: A heading body \\
of message indicating the \\
business of the message. \\
Languge: Informal \\
announcement but \\
professional.
\end{tabular} & Any time. & Outlook. Any place. \\
\hline $\begin{array}{c}\text { Cluster meeting - Marketing } \\
\text { initiatives }\end{array}$ & $\begin{array}{l}\text { Communicate members } \\
\text { about the tean's success in } \\
\text { being short-listed. }\end{array}$ & $\begin{array}{l}\text { Name of } \\
\text { country/organisation, name } \\
\text { of project, names of cluster } \\
\text { members who are involved, } \\
\text { description of current } \\
\text { situation. }\end{array}$ & $\begin{array}{l}\text { Lead company, cluster } \\
\text { Facilitator, cluster } \\
\text { members. }\end{array}$ & $\begin{array}{l}\text { Medium: Face to face } \\
\text { meeting, telephone } \\
\text { conferencing. Structure: } \\
\text { Structured, brief progress } \\
\text { report. Linguistics: } \\
\text { Informal, conversational. }\end{array}$ & $\begin{array}{l}\text { As part of the cluster } \\
\text { meeting, approx. 5-10 } \\
\text { minutes. Same time. }\end{array}$ & $\begin{array}{l}\text { Usually at Positively } \\
\text { Wellington Business } \\
\text { (PWB). Same place. If one } \\
\text { cluster member is on the } \\
\text { phone, then it is different } \\
\text { places. }\end{array}$ \\
\hline
\end{tabular}

Table E.14. Genres in the sub-system R.14 \& P.7 "Decide whether to continue bid or not".

\begin{tabular}{|c|c|c|c|c|c|c|}
\hline Genre & Purpose & Content & Actors (i) & Form & Time & Place \\
\hline $\begin{array}{c}\text { Tender Terms of Reference } \\
\text { (Reactive Approach) }\end{array}$ & $\begin{array}{l}\text { Instruct what needs to be } \\
\text { submited for the tender } \\
\text { process. }\end{array}$ & $\begin{array}{l}\text { Defined guidelines for the } \\
\text { technical proposal. There } \\
\text { are formats for the CVs and } \\
\text { the strucuture of the report. } \\
\text { Details about the scope of } \\
\text { work, methodology. They } \\
\text { also describe the marking } \\
\text { criteria for the tender. }\end{array}$ & Client, lead company. & $\begin{array}{l}\text { Medium: Document; it is } \\
\text { sent as an attachment via } \\
\text { email. Structure: Highly } \\
\text { structured. Linguistics: } \\
\text { Formal, highly technical and } \\
\text { contractual. }\end{array}$ & $\begin{array}{l}\text { Within the tender's } \\
\text { deadline. Any time within } \\
\text { that frame. }\end{array}$ & Any place. \\
\hline Decide to Continue Meeting & $\begin{array}{l}\text { Decide whether or not to } \\
\text { proceed to full tender. }\end{array}$ & $\begin{array}{l}\text { Re-visit the issue of } \\
\text { whether the project is going } \\
\text { to be profitatable enough to } \\
\text { justify the risk and cost of } \\
\text { preparing a tender. } \\
\text { Thorough examination of } \\
\text { the tender's Terms of } \\
\text { Reference to make sure it is } \\
\text { a viable job. Make sure that } \\
\text { the clients are going in the } \\
\text { right direction in terms of } \\
\text { methodology and approach. }\end{array}$ & $\begin{array}{l}\text { Team leader, team } \\
\text { members. }\end{array}$ & $\begin{array}{l}\text { Medium: Face to face } \\
\text { meeting. Structure: } \\
\text { Informal, discussion. } \\
\text { Linguistics: Informal, } \\
\text { conversation. }\end{array}$ & Same time. & $\begin{array}{l}\text { At one of the interested } \\
\text { parties. Same place. }\end{array}$ \\
\hline $\begin{array}{l}\text { Reply to Client (proactive } \\
\text { approach) }\end{array}$ & $\begin{array}{l}\text { Inform clients whether or } \\
\text { not the team will go for } \\
\text { tender. }\end{array}$ & $\begin{array}{l}\text { Greetings, confirm that the } \\
\text { team will continue the } \\
\text { tender process/inform that } \\
\text { the team will not continue } \\
\text { the tender process, } \\
\text { farewell. }\end{array}$ & Lead company, client. & \begin{tabular}{|l|} 
Medium: Email. \\
Structure: Greetings, \\
reasons for writing the email \\
and farewell. \\
Linguistics: Informal but \\
professional.
\end{tabular} & $\begin{array}{l}\text { Within the tender's } \\
\text { deadline. Any time within } \\
\text { that frame. }\end{array}$ & Any place. \\
\hline
\end{tabular}


Table E.15. Genres in the sub-system R.15 \& P.9 "Confirm team for the bid/proposal."

\begin{tabular}{|c|c|c|c|c|c|c|}
\hline Genre & Purpose & Content & Actors (i) & Form & Time & Place \\
\hline Recruiting Team Call & $\begin{array}{l}\text { Ask additional members (or } \\
\text { non-cluster members) to } \\
\text { join the team. }\end{array}$ & $\begin{array}{l}\text { Mention the project and } \\
\text { indicate the potential fit for } \\
\text { the types of services offered } \\
\text { by the new recruit. Request } \\
\text { for CV. }\end{array}$ & Team leader, new recruit. & $\begin{array}{l}\text { Medium: Telephone call. } \\
\text { Structure: Greetings, } \\
\text { reasons for calling, and } \\
\text { farewell. Linguistics: } \\
\text { Informal but professional. }\end{array}$ & $\begin{array}{l}\text { Within the tender/proposal } \\
\text { deadline (WB tenders are } \\
\text { usually } 4 \text { weeks). Any time } \\
\text { within that frame. }\end{array}$ & $\begin{array}{l}\text { Telephone lines. Member's } \\
\text { office or mobile phone. Any } \\
\text { place. }\end{array}$ \\
\hline Recruiting Team Email & $\begin{array}{l}\text { Ask additional members (or } \\
\text { non-cluster members) to } \\
\text { join the team. }\end{array}$ & $\begin{array}{l}\text { A brief message indicating } \\
\text { that a team has been } \\
\text { formed to prepare a } \\
\text { tender/proposal. Show the } \\
\text { potential fit for the types of } \\
\text { services that the new recruit } \\
\text { offers, and invite him/her to } \\
\text { join the team. Request for } \\
\text { cV. }\end{array}$ & Team leader, new recruit. & $\begin{array}{l}\text { Medium: Email. } \\
\text { Structure: A greeting, body } \\
\text { of message indicating the } \\
\text { reason for writing, farewell. } \\
\text { Linguistics: Informal } \\
\text { invitation but professional. }\end{array}$ & $\begin{array}{l}\text { Within the tender/proposal } \\
\text { deadline (WB tenders are } \\
\text { usually } 4 \text { weeks). Any time } \\
\text { within that frame. }\end{array}$ & Outlook. Any place. \\
\hline $\mathrm{CV}$ & $\begin{array}{l}\text { Provide information about } \\
\text { the individual in order to be } \\
\text { considered for the project. } \\
\text { To select the team for the } \\
\text { tender/proposal. }\end{array}$ & $\begin{array}{l}\text { Personal information, } \\
\text { education, work experience, } \\
\text { areas of expertise, } \\
\text { experience, capabilities and } \\
\text { contact details. }\end{array}$ & $\begin{array}{l}\text { Candidate team member, } \\
\text { team leader. }\end{array}$ & $\begin{array}{l}\text { Medium: Email, usually } \\
\text { forwarding the CV. } \\
\text { Structure: Highly } \\
\text { structured; subheadings for } \\
\text { each set of information, } \\
\text { such as personal details, } \\
\text { education, training, } \\
\text { experience, etc. } \\
\text { Linguistics: Formal and } \\
\text { professional. }\end{array}$ & $\begin{array}{l}\text { Within the tender/proposal } \\
\text { deadline (WB tenders are } \\
\text { usually } 4 \text { weeks). Any time } \\
\text { within that frame. }\end{array}$ & Outlook. Any place. \\
\hline Team Leader Call & $\begin{array}{l}\text { Inform who is going to be } \\
\text { part of the tender team. }\end{array}$ & $\begin{array}{l}\text { Communication of who is } \\
\text { going to be part of the } \\
\text { tender/proposal team. }\end{array}$ & $\begin{array}{l}\text { Team leader, candidate } \\
\text { team members. }\end{array}$ & $\begin{array}{l}\text { Medium: Telephone call. } \\
\text { Structure: Greetings, } \\
\text { reasons for calling, and } \\
\text { farewell. Linguistics: } \\
\text { Informal but professional. }\end{array}$ & $\begin{array}{l}\text { Within the tender/proposal } \\
\text { deadline (WB tenders are } \\
\text { usually } 4 \text { weeks). Any time } \\
\text { within that frame. }\end{array}$ & $\begin{array}{l}\text { Telephone lines. Member's } \\
\text { office or mobile phone. Any } \\
\text { place. }\end{array}$ \\
\hline
\end{tabular}

Table E.16. Genres in the sub-system R.16 \& P.10 "Confirm local partner."

\begin{tabular}{|c|c|c|c|c|c|c|}
\hline Genre & Purpose & Content & Actors (i) & Form & Time & Place \\
\hline Call to Local Partner & $\begin{array}{l}\text { Confirm that local partner/s } \\
\text { is/are part of the team. }\end{array}$ & $\begin{array}{l}\text { Greetings, discussion about } \\
\text { their commitment to the bid } \\
\text { and other matters related to } \\
\text { the project, farewell. }\end{array}$ & Team leader, local partner. & $\begin{array}{l}\text { Medium: Telephone call. } \\
\text { Structure: Greetings, } \\
\text { reasons for calling, and } \\
\text { farewell. Linguistics: } \\
\text { Friendly but professional. }\end{array}$ & $\begin{array}{l}\text { Within the tender's deadline } \\
\text { (usually } 4 \text { weeks). Any time } \\
\text { within that frame. }\end{array}$ & $\begin{array}{l}\text { Telephone lines. Member's } \\
\text { office or mobile phone. Any } \\
\text { place. }\end{array}$ \\
\hline Message to Local Partner & $\begin{array}{l}\text { Confirm that local partner/s } \\
\text { is/are still interested in } \\
\text { being part of the team. }\end{array}$ & $\begin{array}{l}\text { Greetings, discussion about } \\
\text { their commitment to the bid } \\
\text { and other matters related to } \\
\text { the project, farewell. }\end{array}$ & Team leader, local partner. & $\begin{array}{l}\text { Medium: Email (sometimes } \\
\text { with attachments), fax. } \\
\text { Structure: A greeting, body } \\
\text { of message indicating the } \\
\text { reason for writing. } \\
\text { Linguistics: Informal } \\
\text { announcement, } \\
\text { professional. }\end{array}$ & $\begin{array}{l}\text { Within the tender's deadline } \\
\text { (usually } 4 \text { weeks). Any time } \\
\text { within that frame. }\end{array}$ & Outlook. Any place. \\
\hline
\end{tabular}


Table E.17. Genres in the sub-system R.17 \& P.11 "Site visit."

\begin{tabular}{|c|c|c|c|c|c|c|}
\hline Genre & Purpose & Content & Actors (i) & Form & Time & Place \\
\hline Meeting with Local Partner & $\begin{array}{l}\text { Meet local partner and } \\
\text { develop a relationship. }\end{array}$ & $\begin{array}{l}\text { Talks about their } \\
\text { background, visit to their } \\
\text { premises, showcase their } \\
\text { work, etc. Discussions } \\
\text { about the current project } \\
\text { and other types of projects } \\
\text { that both parties could do } \\
\text { jointly. }\end{array}$ & $\begin{array}{l}\text { Cluster member who is } \\
\text { part of the bidding team } \\
\text { (usually team leader), } \\
\text { local partner. }\end{array}$ & $\begin{array}{l}\text { Medium: Face to face } \\
\text { meeting. Structure: There } \\
\text { is not a strict order, but the } \\
\text { meeting must cover } \\
\text { background of the local } \\
\text { partner, its capability and } \\
\text { capacity, previous jobs, } \\
\text { interest in working with the } \\
\text { cluster, and their role in the } \\
\text { current bid/proposal. } \\
\text { Linguistics: Informal, } \\
\text { conversational; some } \\
\text { technical and business } \\
\text { vocabulary. }\end{array}$ & Same time. & $\begin{array}{l}\text { At the local partner's } \\
\text { premises. Same place. }\end{array}$ \\
\hline $\begin{array}{l}\text { Meeting with Key Decision } \\
\text { Makers }\end{array}$ & $\begin{array}{l}\text { Gaining further } \\
\text { understanding of the } \\
\text { country, the situation and } \\
\text { the project. }\end{array}$ & $\begin{array}{l}\text { Visits to NZTE \& Embassy } \\
\text { staff, officials from the } \\
\text { World Bank, Government } \\
\text { people and any other } \\
\text { relevant contacts. }\end{array}$ & $\begin{array}{l}\text { Cluster member who is } \\
\text { part of the bidding team } \\
\text { (usually team leader), } \\
\text { NZTE and Embassy staff, } \\
\text { key decision makers. }\end{array}$ & $\begin{array}{l}\text { Medium: Face-to-face } \\
\text { meetings. Structure: } \\
\text { Formal, business meeting. } \\
\text { Linguistics: Formal, } \\
\text { business-oriented. }\end{array}$ & Same time. & Same place. \\
\hline $\begin{array}{l}\text { Meeting with Entity Who is } \\
\text { Organising the Tender } \\
\text { (reactive) or Proposal } \\
\text { (proactive) }\end{array}$ & $\begin{array}{l}\text { Gaining further } \\
\text { understanding of the } \\
\text { situation the project and the } \\
\text { people who are handling the } \\
\text { tender. }\end{array}$ & $\begin{array}{l}\text { Visits to the officials who } \\
\text { are handling the selection } \\
\text { process for the tender } \\
\text { (reactive) or proposal } \\
\text { (proactive). }\end{array}$ & $\begin{array}{l}\text { Cluster member who is } \\
\text { part of the bidding team } \\
\text { (usually team leader), } \\
\text { tender officials. }\end{array}$ & $\begin{array}{l}\text { Medium: Face-to-face } \\
\text { meetings. Structure: } \\
\text { Formal, business meeting. } \\
\text { Linguistics: Formal, } \\
\text { business-oriented. }\end{array}$ & Same time. & Same place. \\
\hline Report to Team & $\begin{array}{l}\text { Inform the team back in } \\
\text { New Zealand about any } \\
\text { findings. Ask for feedback. } \\
\text { Request information. }\end{array}$ & $\begin{array}{l}\text { Greetings. Provide a brief } \\
\text { report of the meetings. } \\
\text { Answer questions and } \\
\text { request information if } \\
\text { necessary. }\end{array}$ & \begin{tabular}{|l} 
Cluster member who is \\
part of the bidding team \\
(usually team leader), \\
team leader, cluster \\
Facilitator. \\
\end{tabular} & \begin{tabular}{|l} 
Medium: Telephone \\
conference. Structure: \\
Formal business meeting. \\
Linguistics: Formal, \\
business-oriented.
\end{tabular} & Same time. & $\begin{array}{l}\text { Telephone line. Different } \\
\text { place. }\end{array}$ \\
\hline Request/Report Message & $\begin{array}{l}\text { Communicate the team any } \\
\text { progress of the visit. } \\
\text { Request information. }\end{array}$ & $\begin{array}{l}\text { Greetings. Provide a brief } \\
\text { report of what is happening } \\
\text { during his/her visit and } \\
\text { request information if } \\
\text { necessary. }\end{array}$ & $\begin{array}{l}\text { Team leader, team } \\
\text { member. }\end{array}$ & \begin{tabular}{|l|} 
Medium: Email. \\
Structure: Greetings, \\
reasons for writing the email \\
and farewell. Language: \\
Informal but professional.
\end{tabular} & $\begin{array}{l}\text { Within the tender's } \\
\text { deadline. Any time within } \\
\text { that frame. }\end{array}$ & Outlook. Any place. \\
\hline Reply to Request Message & $\begin{array}{l}\text { Provide additional } \\
\text { information requested by } \\
\text { the member who is abroad. }\end{array}$ & $\begin{array}{l}\text { Greetings. Provide the } \\
\text { requested information. }\end{array}$ & $\begin{array}{l}\text { Team leader, team } \\
\text { member. }\end{array}$ & \begin{tabular}{|l} 
Medium: Email (it may \\
have attachments). \\
Structure: Greetings, \\
reasons for writing the email \\
and farewell. Linguistics: \\
Informal but professional.
\end{tabular} & $\begin{array}{l}\text { Within the tender's } \\
\text { deadline. Any time within } \\
\text { that frame. }\end{array}$ & Outlook. Any place. \\
\hline
\end{tabular}


Table E.18. Genres in the sub-system R.18 \& P.12 "Prepare Prepare tender/proposal (incl. technical component, financial component \& governance component)"'

\begin{tabular}{|c|c|c|c|c|c|c|}
\hline Genre & Purpose & Content & Actors (i) & Form & Time & Place \\
\hline $\begin{array}{l}\text { Tender Terms of Reference } \\
\text { (for proactive approach) }\end{array}$ & $\begin{array}{l}\text { Instruct what needs to be } \\
\text { submited for the tender } \\
\text { process. }\end{array}$ & $\begin{array}{l}\text { Defined guidelines for the } \\
\text { technical proposal. There } \\
\text { are formats for the CVs and } \\
\text { the strucuture of the report. } \\
\text { Details about the scope of } \\
\text { work, methodology. They } \\
\text { also describe the marking } \\
\text { criteria for the tender. }\end{array}$ & $\begin{array}{l}\text { Client, team leader, team } \\
\text { members. }\end{array}$ & $\begin{array}{l}\text { Medium: Document; it is } \\
\text { sent as an attachment via } \\
\text { email. Structure: Highly } \\
\text { structured. Linguistics: } \\
\text { Formal, highly technical and } \\
\text { contractual. }\end{array}$ & $\begin{array}{l}\text { Within the tender's } \\
\text { deadline. Any time within } \\
\text { that frame. }\end{array}$ & Any place. \\
\hline $\begin{array}{c}\text { Prepare Reactive Tender } \\
\text { Meeting }\end{array}$ & $\begin{array}{l}\text { Coordinate the assembly of } \\
\text { the Tender. }\end{array}$ & $\begin{array}{l}\text { Discussion about what to } \\
\text { include in the tender, } \\
\text { methodology, CVs, track } \\
\text { records. Requests from the } \\
\text { team leader for material that } \\
\text { needs to be part of the } \\
\text { tender. }\end{array}$ & $\begin{array}{l}\text { Team leader, team } \\
\text { members. }\end{array}$ & $\begin{array}{l}\text { Medium: Face to face } \\
\text { meeting, phone conference. } \\
\text { Structure: Structured, brief } \\
\text { progress report. } \\
\text { Linguistics: Informal but } \\
\text { professional, conversation. }\end{array}$ & Same time. & $\begin{array}{l}\text { At one of the interested } \\
\text { parties, same place. When } \\
\text { phone conference, different } \\
\text { place. }\end{array}$ \\
\hline CV Template & $\begin{array}{l}\text { Provide a standard CV for } \\
\text { each specialist who is part } \\
\text { of the team. }\end{array}$ & \begin{tabular}{|l|} 
Information about the \\
expert's contact details, \\
work history, record of work \\
in similar projects, \\
education and training.
\end{tabular} & $\begin{array}{l}\text { Team member, team } \\
\text { leader. }\end{array}$ & \begin{tabular}{|l|} 
Medium: Document; it is \\
usually distributed via email. \\
Structure: Very structured, \\
it follows pre-determined \\
headings. Linguistics: \\
Formal, business-oriented.
\end{tabular} & $\begin{array}{l}\text { Within the tender's } \\
\text { deadline. Any time within } \\
\text { that frame. }\end{array}$ & Same place. \\
\hline Track Record Template & $\begin{array}{l}\text { Provide a standard track } \\
\text { record of previous jobs } \\
\text { done by cluster members } \\
\text { who are part of the team. }\end{array}$ & \begin{tabular}{|l} 
Information about work in \\
previous projects with \\
similar characteristics. \\
Some details are: project \\
name, client, value of \\
project, description of \\
project, specific tasks \\
performed in the project.
\end{tabular} & $\begin{array}{l}\text { Team member, team } \\
\text { leader. }\end{array}$ & $\begin{array}{l}\text { Medium: Document; it is } \\
\text { usually distributed via email. } \\
\text { Structure: Very structured, } \\
\text { it follows a template. } \\
\text { Linguistics: Formal, highly } \\
\text { technical business-oriented. }\end{array}$ & $\begin{array}{l}\text { Within the tender's } \\
\text { deadline. Any time within } \\
\text { that frame. }\end{array}$ & Same place. \\
\hline Request Message & $\begin{array}{l}\text { Request team members } \\
\text { further technical or financial } \\
\text { information to complete the } \\
\text { tender documents. }\end{array}$ & $\begin{array}{l}\text { Greetings, request team } \\
\text { members further technical } \\
\text { or financial information to } \\
\text { complete the tender } \\
\text { documents, farewell }\end{array}$ & $\begin{array}{l}\text { Team leader, team } \\
\text { member. }\end{array}$ & $\begin{array}{l}\text { Medium: Email. } \\
\text { Structure: Greetings, } \\
\text { reasons for writing the email } \\
\text { and farewell. Linguistics: } \\
\text { Informal but professional. }\end{array}$ & $\begin{array}{l}\text { Within the tender's } \\
\text { deadline. Any time within } \\
\text { that frame. }\end{array}$ & Outlook. Any place. \\
\hline $\begin{array}{l}\text { Technical Tender } \\
\text { Document (proactive } \\
\text { approach) }\end{array}$ & $\begin{array}{l}\text { Propose how the project is } \\
\text { going to be approach by the } \\
\text { team. }\end{array}$ & \begin{tabular}{|l|} 
Defines the terms of \\
reference for the project, \\
what does the client wants \\
and what is the group's \\
understanding of it. This \\
document details the \\
methodology the team is \\
proposing to follow.
\end{tabular} & $\begin{array}{l}\text { Team leader, team } \\
\text { member. }\end{array}$ & $\begin{array}{l}\text { Medium: Word Document. } \\
\text { Structure: Very structured, } \\
\text { it follows strict templates } \\
\text { outlined by World Bank } \\
\text { standards. Linguistics: } \\
\text { Formal, highly technical } \\
\text { business-oriented. }\end{array}$ & $\begin{array}{l}\text { Within the tender's } \\
\text { deadline. Any time within } \\
\text { that frame. }\end{array}$ & Same place. \\
\hline $\begin{array}{c}\text { Financial Tender Document } \\
\text { (proactive approach) }\end{array}$ & $\begin{array}{l}\text { Details the proposed } \\
\text { budget, costs and taxes } \\
\text { issues for the project. }\end{array}$ & Tables & $\begin{array}{l}\text { Team leader, team } \\
\text { member. }\end{array}$ & $\begin{array}{l}\text { Medium: Word Document } \\
\text { or Excel file; it could be sent } \\
\text { as attachment via email. } \\
\text { Structure: Very structured, } \\
\text { team has to filled financial } \\
\text { tables outlined by World } \\
\text { Bank standards. } \\
\text { Linguistics: Formal, highly } \\
\text { financial and business- } \\
\text { oriented. }\end{array}$ & $\begin{array}{l}\text { Within the tender's } \\
\text { deadline. Any time within } \\
\text { that frame. }\end{array}$ & Same place. \\
\hline Proposal & $\begin{array}{l}\text { Propose how the project is } \\
\text { going to be approach by the } \\
\text { team and its financial cost. }\end{array}$ & $\begin{array}{l}\text { Defines the terms of } \\
\text { reference for the project, } \\
\text { what does the client want } \\
\text { and what is the group's } \\
\text { understanding of it. This } \\
\text { document has two main } \\
\text { components: technical, } \\
\text { which details the } \\
\text { methodology the team is } \\
\text { proposing to follow; and the } \\
\text { financial, which details the } \\
\text { proposed budget, costs and } \\
\text { taxes issues for the project. }\end{array}$ & $\begin{array}{l}\text { Team leader and cluster } \\
\text { members who are part of } \\
\text { the bidding team. }\end{array}$ & \begin{tabular}{|l|} 
Medium: Word Document. \\
Structure: Very structured, \\
it follows defined templates \\
outlined by the lead \\
company. Linguistics: \\
Formal, highly technical, \\
contractual, business- \\
oriented.
\end{tabular} & $\begin{array}{l}\text { Within the proposal's } \\
\text { deadline. Any time within } \\
\text { that frame. }\end{array}$ & Same place. \\
\hline $\begin{array}{l}\text { Governance Tender } \\
\text { Agreement }\end{array}$ & $\begin{array}{l}\text { Seek agreement on the } \\
\text { governance structure that } \\
\text { the team will follow }\end{array}$ & $\begin{array}{l}\text { Agreement on: who is going } \\
\text { to be the lead company, the } \\
\text { proportion of work that each } \\
\text { member will take on, } \\
\text { sharing of costs, liability and } \\
\text { profits. }\end{array}$ & $\begin{array}{l}\text { Team leader, team } \\
\text { member. }\end{array}$ & $\begin{array}{l}\text { Medium: Letter } \\
\text { Structure: Structured, } \\
\text { contractual. Linguistics: } \\
\text { Formal, contractual. }\end{array}$ & $\begin{array}{l}\text { Within the tender's } \\
\text { deadline. Any time within } \\
\text { that frame. }\end{array}$ & Any place. \\
\hline
\end{tabular}


Table E.19. Genres in the sub-system R.19 \& P.13 "Delivery of tender/proposal."

\begin{tabular}{|c|c|c|c|c|c|c|}
\hline Genre & Purpose & Content & Actors (i) & Form & Time & Place \\
\hline Covers of Tender & $\begin{array}{l}\text { Inform the client the title of } \\
\text { the document and who are } \\
\text { the authors. }\end{array}$ & $\begin{array}{l}\text { Title of the document, } \\
\text { authors, date, pictures and } \\
\text { graphic design. }\end{array}$ & \begin{tabular}{|l} 
Team leader, local \\
partners, client.
\end{tabular} & \begin{tabular}{|l|} 
Medium: Printed pages \\
sent via courier. Structure: \\
Very structured, it follows a \\
template. Linguistics: \\
Formal, highly technical and \\
business-oriented.
\end{tabular} & $\begin{array}{l}\text { Within the tender's } \\
\text { deadline. Any time within } \\
\text { that frame. }\end{array}$ & Same place. \\
\hline Original Signed Documents & Guarantee authenticity. & Set of official documents. & $\begin{array}{l}\text { Team leader, local } \\
\text { partners, client. }\end{array}$ & $\begin{array}{l}\text { Medium: Printed document } \\
\text { sent via courier. Structure: } \\
\text { Very structured, letter. } \\
\text { Linguistics: Formal. }\end{array}$ & Any time. & Same place. \\
\hline $\begin{array}{l}\text { Message with Technical } \\
\text { and Financial Documents } \\
\text { (proactive approach) }\end{array}$ & $\begin{array}{l}\text { Send the electronic version } \\
\text { of the Technical and } \\
\text { Financial Documents to the } \\
\text { local partners or Trade } \\
\text { Comission. }\end{array}$ & $\begin{array}{l}\text { Brief message with the } \\
\text { Technical Document } \\
\text { attached. }\end{array}$ & $\begin{array}{l}\text { Team leader, local } \\
\text { partners, Trade Comission, } \\
\text { client. }\end{array}$ & $\begin{array}{l}\text { Medium: Email with } \\
\text { Technical and Financial } \\
\text { documents attached. } \\
\text { Structure: Formal. } \\
\text { Linguistics: Formal. } \\
\end{array}$ & $\begin{array}{l}\text { Within the tender's } \\
\text { deadline. Any time within } \\
\text { that frame. }\end{array}$ & Outlook. Any place. \\
\hline $\begin{array}{l}\text { Message with Proposal } \\
\text { (reactive approach) }\end{array}$ & $\begin{array}{l}\text { Send the electronic version } \\
\text { of the Technical Document } \\
\text { to the local partners or } \\
\text { Trade Comission. }\end{array}$ & $\begin{array}{l}\text { Brief message with the } \\
\text { Technical Document } \\
\text { attached. }\end{array}$ & $\begin{array}{l}\text { Team leader, local } \\
\text { partners, Trade Comission, } \\
\text { client. }\end{array}$ & $\begin{array}{l}\text { Medium: Email with } \\
\text { Proposal attached. } \\
\text { Structure: Formal. } \\
\text { Linguistics: Formal. }\end{array}$ & $\begin{array}{l}\text { Within the tender's } \\
\text { deadline. Any time within } \\
\text { that frame. }\end{array}$ & Outlook. Any place. \\
\hline Call to Local Partner & $\begin{array}{l}\text { Check progress on delivery } \\
\text { of both envelopes (technical } \\
\text { and financial). }\end{array}$ & $\begin{array}{l}\text { Brief to check if } \\
\text { documentation has arrived. }\end{array}$ & $\begin{array}{l}\text { Team leader, local } \\
\text { partners, Trade Comission, } \\
\text { client. }\end{array}$ & $\begin{array}{l}\text { Medium: Telephone call. } \\
\text { Structure: Greetings, } \\
\text { reasons for calling, and } \\
\text { farewell. Linguistics: } \\
\text { Informal but professional. }\end{array}$ & $\begin{array}{l}\text { Within the tender's } \\
\text { deadline. Any time within } \\
\text { that frame. }\end{array}$ & $\begin{array}{l}\text { Telephone lines. Lead } \\
\text { company's office or mobile } \\
\text { phone. Any place. }\end{array}$ \\
\hline
\end{tabular}

Table E.20. Genres in the sub-system R.20 \& P.14 "Follow-up tender/proposal".

\begin{tabular}{|c|c|c|c|c|c|c|}
\hline Genre & Purpose & Content & Actors (i) & Form & Time & Place \\
\hline Follow-up Visit & $\begin{array}{l}\text { Find out how the selection } \\
\text { process is coming along. }\end{array}$ & $\begin{array}{l}\text { Informal visits to the } \\
\text { officials who are handling } \\
\text { the selection process for the } \\
\text { tender/proposal. }\end{array}$ & $\begin{array}{l}\text { Lead company, local } \\
\text { partners, NZTE and } \\
\text { Embassy staff, cluster } \\
\text { members who are visiting } \\
\text { the market. }\end{array}$ & $\begin{array}{l}\text { Medium: Face-to-face } \\
\text { meetings. } \\
\text { Structure: Informal, casual. } \\
\text { Linguistics: Casual, } \\
\text { business-oriented. }\end{array}$ & Same time. & Same place. \\
\hline Follow-up Calls & $\begin{array}{l}\text { Find out how the selection } \\
\text { process is coming along. }\end{array}$ & $\begin{array}{l}\text { Discussing and inquiring } \\
\text { any information about the } \\
\text { progress of the selection } \\
\text { process. }\end{array}$ & $\begin{array}{l}\text { Lead company, local } \\
\text { partners, NZTE and } \\
\text { Embassy staff, cluster } \\
\text { Facilitator. }\end{array}$ & $\begin{array}{l}\text { Medium: Telephone calls. } \\
\text { Structure: Informal, casual. } \\
\text { Linguistics: Casual, } \\
\text { business-oriented. }\end{array}$ & Same time. & $\begin{array}{l}\text { Telephone lines. Different } \\
\text { places. }\end{array}$ \\
\hline Follow-up Messages & $\begin{array}{l}\text { Find out how the selection } \\
\text { process is coming along. }\end{array}$ & $\begin{array}{l}\text { Discussing and inquiring } \\
\text { any information about the } \\
\text { progress of the selection } \\
\text { process. }\end{array}$ & $\begin{array}{l}\text { Lead company, local } \\
\text { partners, NZTE and } \\
\text { Embassy staff, cluster } \\
\text { Facilitator. }\end{array}$ & $\begin{array}{l}\text { Medium: Email. } \\
\text { Structure: Informal, casual. } \\
\text { Linguistics: Casual, } \\
\text { business-oriented. }\end{array}$ & Different time. & Outlook. Different places. \\
\hline
\end{tabular}

Table E.21. Genres in the sub-system R.21 \& P.15 "Tender/Proposal is selected."

\begin{tabular}{|c|c|c|c|c|c|c|}
\hline Genre & Purpose & Content & Actors (i) & Form & Time & Place \\
\hline $\begin{array}{l}\text { Opening of Financial } \\
\text { Envelope (Reactive } \\
\text { Approach) }\end{array}$ & $\begin{array}{l}\text { Open the financial } \\
\text { envelopes of those } \\
\text { candidates who have } \\
\text { passed the technical criteria } \\
\text { in a public meeting. }\end{array}$ & $\begin{array}{l}\text { The envelopes are opened } \\
\text { and the prices are read out } \\
\text { loud. }\end{array}$ & $\begin{array}{l}\text { Client, local partners, } \\
\text { cluster representative, } \\
\text { officials handling the tender. }\end{array}$ & $\begin{array}{l}\text { Medium: Face-to-face } \\
\text { meetings. Structure: } \\
\text { Formal. Linguistics: } \\
\text { Formal, contractual. }\end{array}$ & Same time. & Same place. \\
\hline $\begin{array}{l}\text { Notification to Lead } \\
\text { Company }\end{array}$ & $\begin{array}{l}\text { Inform the lead company } \\
\text { that his/her team has been } \\
\text { selected. }\end{array}$ & $\begin{array}{l}\text { Inform the lead company } \\
\text { that his/her team has been } \\
\text { selected and details for the } \\
\text { next stage. }\end{array}$ & Client, team member & $\begin{array}{l}\text { Medium: Letter sent via } \\
\text { email. Structure: } \\
\text { Structured Linguistics: } \\
\text { Formal }\end{array}$ & Any time. & Any place. \\
\hline Announcement Calls & $\begin{array}{l}\text { Communicate the results of } \\
\text { the selection process to the } \\
\text { rest of the team. }\end{array}$ & $\begin{array}{l}\text { Results of the selection } \\
\text { process to the rest of the } \\
\text { team. }\end{array}$ & $\begin{array}{l}\text { Team leader, local partner, } \\
\text { team members. }\end{array}$ & $\begin{array}{l}\text { Medium: Telephone calls. } \\
\text { Structure: Informal, casual. } \\
\text { Linguistics: Casual, } \\
\text { business-oriented. }\end{array}$ & Same time. & $\begin{array}{l}\text { Telephone lines. Different } \\
\text { place. }\end{array}$ \\
\hline Announcement Message & $\begin{array}{l}\text { Communicate the results of } \\
\text { the selection process to the } \\
\text { rest of the team. }\end{array}$ & $\begin{array}{l}\text { Results of the selection } \\
\text { process to the rest of the } \\
\text { team. }\end{array}$ & $\begin{array}{l}\text { Local partners, NZTE and } \\
\text { Embassy staff, cluster } \\
\text { Facilitator, lead company. }\end{array}$ & $\begin{array}{l}\text { Medium: Email. } \\
\text { Structure: Informal, casual. } \\
\text { Linguistics: Casual, } \\
\text { business-oriented. }\end{array}$ & Different time. & Outlook. Different place. \\
\hline Announcement Memo & $\begin{array}{l}\text { Communicate the results of } \\
\text { the selection process to the } \\
\text { rest of the cluster. }\end{array}$ & $\begin{array}{l}\text { Memo indicating that the } \\
\text { team has been successful } \\
\text { in winning the bid/proposal. }\end{array}$ & $\begin{array}{l}\text { Team leader, cluster } \\
\text { facilitator, PWB Liaison, } \\
\text { cluster members. }\end{array}$ & $\begin{array}{l}\text { Medium: Email. } \\
\text { Structure: A greeting, body } \\
\text { of message indicating the } \\
\text { business of the message, a } \\
\text { farewell. Linguistics: } \\
\text { Informal announcement, } \\
\text { professional. }\end{array}$ & Any time. & Outlook. Any place. \\
\hline
\end{tabular}

(i) The highlighted actors are the ones who usually initiate the genre. 
Table E.22. Genres in the sub-system R.22 \& P.16 "Negotiate final contract."

\begin{tabular}{|c|c|c|c|c|c|c|}
\hline Genre & Purpose & Content & Actors (i) & Form & Time & Place \\
\hline Negotiation Meeting & $\begin{array}{l}\text { To reach final agreement on } \\
\text { the composition of the } \\
\text { contract. }\end{array}$ & $\begin{array}{l}\text { Discussions about } \\
\text { including, eliminating or } \\
\text { changing some aspects of } \\
\text { the proposals. Price may } \\
\text { also be discussed. }\end{array}$ & Team leader, client. & \begin{tabular}{|l} 
Medium: Face-to-face \\
meetings. Structure: \\
Formal. Linguistics: \\
Formal, contractual.
\end{tabular} & Same place. & Same time. \\
\hline Negotiation Message & $\begin{array}{l}\text { To reach final agreement on } \\
\text { the composition of the } \\
\text { contract. }\end{array}$ & $\begin{array}{l}\text { Discussions about } \\
\text { including, eliminating or } \\
\text { changing some aspects of } \\
\text { the proposals. Price may } \\
\text { also be discussed. }\end{array}$ & Team leader, client. & $\begin{array}{l}\text { Medium: Email with drafts } \\
\text { of documents attached. } \\
\text { Structure: Formal. } \\
\text { Linguistics: Formal, } \\
\text { contractual. } \\
\end{array}$ & Any place. & Any time. \\
\hline $\begin{array}{l}\text { Informing the Team } \\
\text { Message }\end{array}$ & $\begin{array}{l}\text { Communicate the team that } \\
\text { final agreement has been } \\
\text { reached. }\end{array}$ & $\begin{array}{l}\text { Greetings, announcing that } \\
\text { final agreement with client } \\
\text { has been reached and } \\
\text { signing of contract will take } \\
\text { place shortly, farewell. }\end{array}$ & $\begin{array}{l}\text { Team leader, cluster } \\
\text { members involved in the } \\
\text { team. }\end{array}$ & $\begin{array}{l}\text { Medium: Email. } \\
\text { Structure: Greetings, } \\
\text { reasons for writing the email } \\
\text { and farewell. Linguistics: } \\
\text { Informal but professional. }\end{array}$ & Any time. & Outlook. Any place. \\
\hline Short-list Memo & $\begin{array}{l}\text { Communicate to the rest of } \\
\text { the cluster that negotiations } \\
\text { have been succesful. }\end{array}$ & $\begin{array}{l}\text { Heading with title of the } \\
\text { project. Announcement of } \\
\text { the good news and } \\
\text { informing members that } \\
\text { further details would be } \\
\text { discussed in the next } \\
\text { cluster meeting. }\end{array}$ & $\begin{array}{l}\text { Cluster Facilitator, team } \\
\text { leader, cluster members. }\end{array}$ & $\begin{array}{l}\text { Medium: Email. } \\
\text { Structure: A heading body } \\
\text { of message indicating the } \\
\text { business of the message. } \\
\text { Linguistics: Informal } \\
\text { announcement but } \\
\text { professional. }\end{array}$ & Any time. & Outlook. Any place. \\
\hline $\begin{array}{c}\text { Cluster meeting - Marketing } \\
\text { initiatives }\end{array}$ & $\begin{array}{l}\text { Communicate to members } \\
\text { about the team's success in } \\
\text { the final negotiations for the } \\
\text { project. }\end{array}$ & $\begin{array}{l}\text { Name of } \\
\text { country/organisation, name } \\
\text { of project, names of cluster } \\
\text { members who are involved, } \\
\text { description of current } \\
\text { situation. }\end{array}$ & $\begin{array}{l}\text { Lead company, cluster } \\
\text { Facilitator, cluster } \\
\text { members. }\end{array}$ & $\begin{array}{l}\text { Medium: Face to face } \\
\text { meeting, phone } \\
\text { conferencing. Structure: } \\
\text { Structured, brief progress } \\
\text { report. Linguistics: } \\
\text { Informal, conversational. }\end{array}$ & $\begin{array}{l}\text { As part of the cluster } \\
\text { meeting, approx. } 5-10 \\
\text { minutes. Same time. }\end{array}$ & $\begin{array}{l}\text { Usually at Positively } \\
\text { Wellington Business } \\
\text { (PWB). Same place. If one } \\
\text { cluster member is on the } \\
\text { phone, then it is different } \\
\text { places. }\end{array}$ \\
\hline
\end{tabular}

Table E.23. The sub-system R.23 \& P.17 "Project starts" (*).

\begin{tabular}{|c|c|c|c|c|c|c|}
\hline Genre & Purpose & Content & Actors & Form & Time & Place \\
\hline & & & & & & \\
\hline
\end{tabular}

Table E.24. The sub-system R.24 \& P. 18 "Project finishes" (*).

\begin{tabular}{|c|c|c|c|c|c|c|}
\hline Genre & Purpose & Content & Actors & Form & Time & Place \\
\hline & & & & & & \\
\hline
\end{tabular}

(*) This stage was included in the project bidding process to illustrate the start/end of the job. As such, there are no communication interactions and therefore no Genre Systems analysis was done to it. 
Table E.25. Genres in the sub-system R.25 \& P.1 "Building relationships."

\begin{tabular}{|c|c|c|c|c|c|c|}
\hline Genre & Purpose & Content & Actors (i) & Form & Time & Place \\
\hline $\begin{array}{l}\text { Meeting with World } \\
\text { BankiADESSOPAC key } \\
\text { officials }\end{array}$ & $\begin{array}{l}\text { Raise the cluster's profile. } \\
\text { Get to know the official. }\end{array}$ & $\begin{array}{l}\text { Discussions about the } \\
\text { cluster's capability and } \\
\text { capacity, experience and } \\
\text { track record. Learn about } \\
\text { the official and hisher } \\
\text { key role. }\end{array}$ & $\begin{array}{l}\text { Cluster members who } \\
\text { are visiting these } \\
\text { organisations, World } \\
\text { BankiADB/Sopac } \\
\text { officials. }\end{array}$ & $\begin{array}{l}\text { Medium: Face-to-face } \\
\text { meeting. Structure: } \\
\text { Friendly but professional. } \\
\text { Linguistics: Formal. }\end{array}$ & Same time. & $\begin{array}{l}\text { At the officials' premises } \\
\text { Same place. }\end{array}$ \\
\hline $\begin{array}{c}\text { Message to World } \\
\text { BankiADBSOSOPAC key } \\
\text { officials }\end{array}$ & $\begin{array}{l}\text { Keep contact with local } \\
\text { World BankiADEISOPAC } \\
\text { key officials. Build a } \\
\text { relationship. }\end{array}$ & $\begin{array}{l}\text { Discussions about the } \\
\text { cluster's capability and } \\
\text { capacity, experience and } \\
\text { track record. Learn about } \\
\text { the official and hisher } \\
\text { key role. }\end{array}$ & $\begin{array}{l}\text { Cluster members, } \\
\text { cluster facilitator, } \\
\text { World Bank/ADEISOPAC } \\
\text { key officials. }\end{array}$ & $\begin{array}{l}\text { Medium: Email. } \\
\text { Structure: A greeting, } \\
\text { body of message } \\
\text { indicating the reason for } \\
\text { writing. Linguistics: } \\
\text { Informal, professional. }\end{array}$ & Any time & Outlook. Any place. \\
\hline $\begin{array}{c}\text { Call to World } \\
\text { BankiAaBDSOPAC key } \\
\text { officials }\end{array}$ & $\begin{array}{l}\text { Keep contact with local } \\
\text { World Bank/ADE/SOPAC } \\
\text { key officials. Build a } \\
\text { relationship. }\end{array}$ & $\begin{array}{l}\text { Discussions about the } \\
\text { cluster's capability and } \\
\text { capacity, experience and } \\
\text { track record. Learn about } \\
\text { the official and hisher } \\
\text { key role. }\end{array}$ & $\begin{array}{l}\text { Cluster members, } \\
\text { cluster Facilitator, } \\
\text { World Bank/ADEISOPAC } \\
\text { key officials. }\end{array}$ & $\begin{array}{l}\text { Medium: Telephone call. } \\
\text { Structure: Greetings, } \\
\text { reasons for calling, and } \\
\text { farewell. Linguistics: } \\
\text { Informal but professional. }\end{array}$ & Any time & $\begin{array}{l}\text { Telephone lines. Any } \\
\text { place. }\end{array}$ \\
\hline Meeting with Clients & $\begin{array}{l}\text { Meet local clients. Build a } \\
\text { relationship. }\end{array}$ & Depends on the situation. & $\begin{array}{l}\text { Team leader, cluster } \\
\text { member who is visiting } \\
\text { the market, clients. }\end{array}$ & $\begin{array}{l}\text { Medium: Face to face } \\
\text { meeting. } \\
\text { Structure:Depends on } \\
\text { the situation. } \\
\text { Linguistics: Informal, } \\
\text { conversational; some } \\
\text { technical and business } \\
\text { vocabulary. } \\
\end{array}$ & Same time. & Same place. \\
\hline Message to Clients & $\begin{array}{l}\text { Keep contact with local } \\
\text { client. Build a } \\
\text { relationship. }\end{array}$ & Depends on the situation. & Team leader, client. & \begin{tabular}{|l|} 
Medium: Email. \\
Structure: A greeting, \\
body of message \\
indicating the reason for \\
writing. Linguistics: \\
Friendly but professional.
\end{tabular} & Any time & Outlook. Any place. \\
\hline Call to Clients & $\begin{array}{l}\text { Keep contact with local } \\
\text { client. Build a } \\
\text { relationship. }\end{array}$ & & $\begin{array}{l}\text { Cluster members, } \\
\text { client. }\end{array}$ & $\begin{array}{l}\text { Medium:Telephone call. } \\
\text { Structure: Greetings, } \\
\text { reasons for calling, and } \\
\text { farewell. Linguistics: } \\
\text { Informal but professional. }\end{array}$ & Any time & $\begin{array}{l}\text { Telephone lines. Any } \\
\text { place. }\end{array}$ \\
\hline $\begin{array}{l}\text { Meeting with Local } \\
\text { Partner }\end{array}$ & $\begin{array}{l}\text { Meet local partner and } \\
\text { assess how established, } \\
\text { credible and connected } \\
\text { they are. Build a } \\
\text { relationship. }\end{array}$ & $\begin{array}{l}\text { Talks about their } \\
\text { background, visit to their } \\
\text { prenises, showcase their } \\
\text { work, etc. Discussions } \\
\text { about types of projects } \\
\text { that both parties could do } \\
\text { jointly. }\end{array}$ & $\begin{array}{l}\text { Local partner, cluster } \\
\text { member who is } \\
\text { visiting the market. }\end{array}$ & $\begin{array}{l}\text { Medium: Face to face } \\
\text { meeting. Structure: } \\
\text { There is not a strict order, } \\
\text { but the meeting must } \\
\text { cover background of the } \\
\text { local partner, its capability } \\
\text { and capacity, previous } \\
\text { jobs, interest in working } \\
\text { with the cluster. } \\
\text { Linguistics: Informal, } \\
\text { conversational; some } \\
\text { technical and business } \\
\text { vocabulary. }\end{array}$ & Same time. & $\begin{array}{l}\text { At the local partner's } \\
\text { premises. Same place. }\end{array}$ \\
\hline Message to Local Partner & $\begin{array}{l}\text { Keep contact with local } \\
\text { partner. Build a } \\
\text { relationship. }\end{array}$ & & $\begin{array}{l}\text { Cluster members, } \\
\text { local partner. }\end{array}$ & \begin{tabular}{|l} 
Medium: Email. \\
Structure: A greeting, \\
body of message \\
indicating the reason for \\
witing. Linguistics: \\
informal, professional. \\
\end{tabular} & Any time & Outlook. Any place. \\
\hline Call to Local Partner & $\begin{array}{l}\text { Keep contact with local } \\
\text { partner. Build a } \\
\text { relationship. }\end{array}$ & & $\begin{array}{l}\text { Cluster members, } \\
\text { local partner. }\end{array}$ & $\begin{array}{l}\text { Medium: Telephone call. } \\
\text { Structure: Greetings, } \\
\text { reasons for calling, and } \\
\text { farewell. Linguistics: } \\
\text { Informal but professional. }\end{array}$ & Any time & $\begin{array}{l}\text { Telephone lines. Any } \\
\text { place. }\end{array}$ \\
\hline $\begin{array}{l}\text { Meeting with NZTE and } \\
\text { Embassy staff }\end{array}$ & $\begin{array}{l}\text { Meet local NZTE officials } \\
\text { and Embassy staff in the } \\
\text { country. Build a } \\
\text { relationship. }\end{array}$ & $\begin{array}{l}\text { Talks about the cluster. } \\
\text { Discussions about } \\
\text { opportunities in the } \\
\text { country. }\end{array}$ & $\begin{array}{l}\text { Cluster member who } \\
\text { is visiting the market, } \\
\text { cluster Facilitator, } \\
\text { NZTE and Embassy staff. }\end{array}$ & $\begin{array}{l}\text { Medium: Face to face } \\
\text { meeting. } \\
\text { Structure: } \\
\text { Linguistics: Infornal, } \\
\text { conversational; some } \\
\text { technical and business } \\
\text { vocabulary. }\end{array}$ & Same time. & $\begin{array}{l}\text { Att the NZTE or Embassy } \\
\text { premises. Same place. }\end{array}$ \\
\hline $\begin{array}{l}\text { Message to NZTE and } \\
\text { Embassy staff }\end{array}$ & $\begin{array}{l}\text { Keep contact with local } \\
\text { NZTE and Embassy staff. } \\
\text { Build a relationship. }\end{array}$ & $\begin{array}{l}\text { Talks about the cluster. } \\
\text { Discussions about } \\
\text { opportunities in the } \\
\text { country. }\end{array}$ & $\begin{array}{l}\text { Cluster members, } \\
\text { cluster Facilitator, } \\
\text { NZTE and Embassy staff. }\end{array}$ & $\begin{array}{l}\text { Medium: Email. } \\
\text { Structure: A greeting, } \\
\text { body of message } \\
\text { indicating the reason for } \\
\text { writing. Linguistics: } \\
\text { Informal, professional. }\end{array}$ & Any time & Outlook. Any place. \\
\hline $\begin{array}{c}\text { Call to NZTE and Embassy } \\
\text { staff }\end{array}$ & $\begin{array}{l}\text { Keep contact with local } \\
\text { NZTE and Embassy staff. } \\
\text { Build a relationship. }\end{array}$ & $\begin{array}{l}\text { Talks about the cluster. } \\
\text { Discussions about } \\
\text { opportunities in the } \\
\text { country. }\end{array}$ & $\begin{array}{l}\text { Cluster members, } \\
\text { cluster Facilitator, } \\
\text { NZTE and Embassy staff. }\end{array}$ & $\begin{array}{l}\text { Medium: Telephone call. } \\
\text { Structure: Greetings, } \\
\text { reasons for calling, and } \\
\text { farewell. Linguistics: } \\
\text { Informal but professional. }\end{array}$ & Any time & $\begin{array}{l}\text { Telephone lines. Any } \\
\text { place. }\end{array}$ \\
\hline Expert Proflie & $\begin{array}{l}\text { Provide information about } \\
\text { a specialist. }\end{array}$ & $\begin{array}{l}\text { Information about the } \\
\text { expert's contact details, } \\
\text { work history, record of } \\
\text { work in similar projects, } \\
\text { education and training. }\end{array}$ & $\begin{array}{l}\text { Team member, team } \\
\text { leader, cluster } \\
\text { Facilitator. }\end{array}$ & $\begin{array}{l}\text { Medium: Document; it is } \\
\text { usually distributed via } \\
\text { email. Structure: Very } \\
\text { structured, it follows pre- } \\
\text { determined headings. } \\
\text { Linguistics: Formal, } \\
\text { business-oriented. }\end{array}$ & $\begin{array}{l}\text { Within the tender's } \\
\text { deadline. Any time within } \\
\text { that frame. }\end{array}$ & Same place. \\
\hline Project Sheet & $\begin{array}{l}\text { Describe a previous job } \\
\text { done by cluster members. }\end{array}$ & $\begin{array}{l}\text { Some details are: project } \\
\text { name, client, value of } \\
\text { project, description of } \\
\text { project, specific tasks } \\
\text { performed in the project. }\end{array}$ & $\begin{array}{l}\text { Team member, team } \\
\text { leader, cluster } \\
\text { Facilitator. }\end{array}$ & $\begin{array}{l}\text { Medium: Document; it is } \\
\text { usually distributed via } \\
\text { email. Structure: Very } \\
\text { structured, it follows a } \\
\text { template. Linguistics: } \\
\text { Formal, highly technical } \\
\text { business-oriented. }\end{array}$ & $\begin{array}{l}\text { Within the tender's } \\
\text { deadline. Any time within } \\
\text { that frame. }\end{array}$ & Same place. \\
\hline Company Brochure & $\begin{array}{l}\text { To showcase the } \\
\text { company and its } \\
\text { capabilities. }\end{array}$ & $\begin{array}{l}\text { Set of glossy material and } \\
\text { brochures describing the } \\
\text { companies and their } \\
\text { capabilities that is } \\
\text { attached to the EOI. }\end{array}$ & $\begin{array}{l}\text { EOI team, local partners, } \\
\text { client. } \\
\end{array}$ & $\begin{array}{l}\text { Medium: Printed } \\
\text { document posted by } \\
\text { courier } \\
\text { Structure: Very } \\
\text { structured, marketing- } \\
\text { type format. } \\
\text { Linguistics: Formal, } \\
\text { technical and } \\
\text { businessinarketing. } \\
\end{array}$ & Any time. & Any place. \\
\hline
\end{tabular}


Table E.26. Genres in the sub-system R.26 \& P.2 "Discussion of other needs with clients."

\begin{tabular}{|c|c|c|c|c|c|c|}
\hline Genre & Purpose & Content & Actors (i) & Form & Time & Place \\
\hline Meeting with Clients & $\begin{array}{l}\text { Meet local clients. Discuss } \\
\text { needs and come up with a } \\
\text { specific proposal. }\end{array}$ & $\begin{array}{l}\text { Discuss needs and come } \\
\text { up with a specific proposal. }\end{array}$ & $\begin{array}{l}\text { Cluster member who is } \\
\text { visiting the market, client. }\end{array}$ & $\begin{array}{l}\text { Medium: Face to face } \\
\text { meeting. } \\
\text { Structure: Linguistics: } \\
\text { Informal, conversational; } \\
\text { some technical and } \\
\text { business vocabulary. }\end{array}$ & Same time. & Same place. \\
\hline Message to Clients & $\begin{array}{l}\text { Discuss further needs and } \\
\text { come up with a specific } \\
\text { proposal. }\end{array}$ & $\begin{array}{l}\text { Follow-up what it was } \\
\text { discussed during the last } \\
\text { meeting. }\end{array}$ & Team leader, client. & $\begin{array}{l}\text { Medium: Email. } \\
\text { Structure: A greeting, body } \\
\text { of message indicating the } \\
\text { reason for writing. } \\
\text { Linguistics: Informal, } \\
\text { professional. }\end{array}$ & Any time & Outlook. Any place. \\
\hline Call to Clients & $\begin{array}{l}\text { Discuss further needs and } \\
\text { come up with a specific } \\
\text { proposal }\end{array}$ & $\begin{array}{l}\text { Follow up what was } \\
\text { discussed during the last } \\
\text { meeting. }\end{array}$ & Team leader, client. & $\begin{array}{l}\text { Medium: Telephone call. } \\
\text { Structure: Greetings, } \\
\text { reasons for calling, and } \\
\text { farewell. Linguistics: } \\
\text { Informal but professional. }\end{array}$ & Any time & $\begin{array}{l}\text { Telephone lines. Any } \\
\text { place. }\end{array}$ \\
\hline
\end{tabular}

